In cooperation with the Texas Natural Resource Conservation Commission

\title{
Volatile Organic Compound and Pesticide Data for Public Water-Supply Reservoirs and Wells, Texas, 1999-2001
}

Open-File Report 02-093

U.S. Department of the Interior

U.S. Geological Survey 
U.S. Department of the Interior

U.S. Geological Survey

\section{Volatile Organic Compound and Pesticide Data for Public Water-Supply Reservoirs and Wells, Texas, 1999-2001}

By B.J. Mahler, M.O. Gary, M.G. Canova, Eric W. Strom, Lynne Fahlquist, and Michael E. Dorsey

\section{U.S. GEOLOGICAL SURVEY}

Open-File Report 02-093

In cooperation with the Texas Natural Resource Conservation Commission

Austin, Texas

2002 


\section{U.S. DEPARTMENT OF THE INTERIOR}

Gale A. Norton, Secretary

\section{U.S. GEOLOGICAL SURVEY}

Charles G. Groat, Director

Any use of trade, product, or firm names is for descriptive purposes only and does not imply endorsement by the U.S. Government.

For additional information write to

\section{District Chief}

U.S. Geological Survey

8027 Exchange Dr.

Austin, TX 78754-4733

E-mail: dc_tx@usgs.gov

Copies of this report can be purchased from

U.S. Geological Survey

Information Services

Box 25286

Denver, CO 80225-0286

E-mail: infoservices@usgs.gov 


\section{CONTENTS}

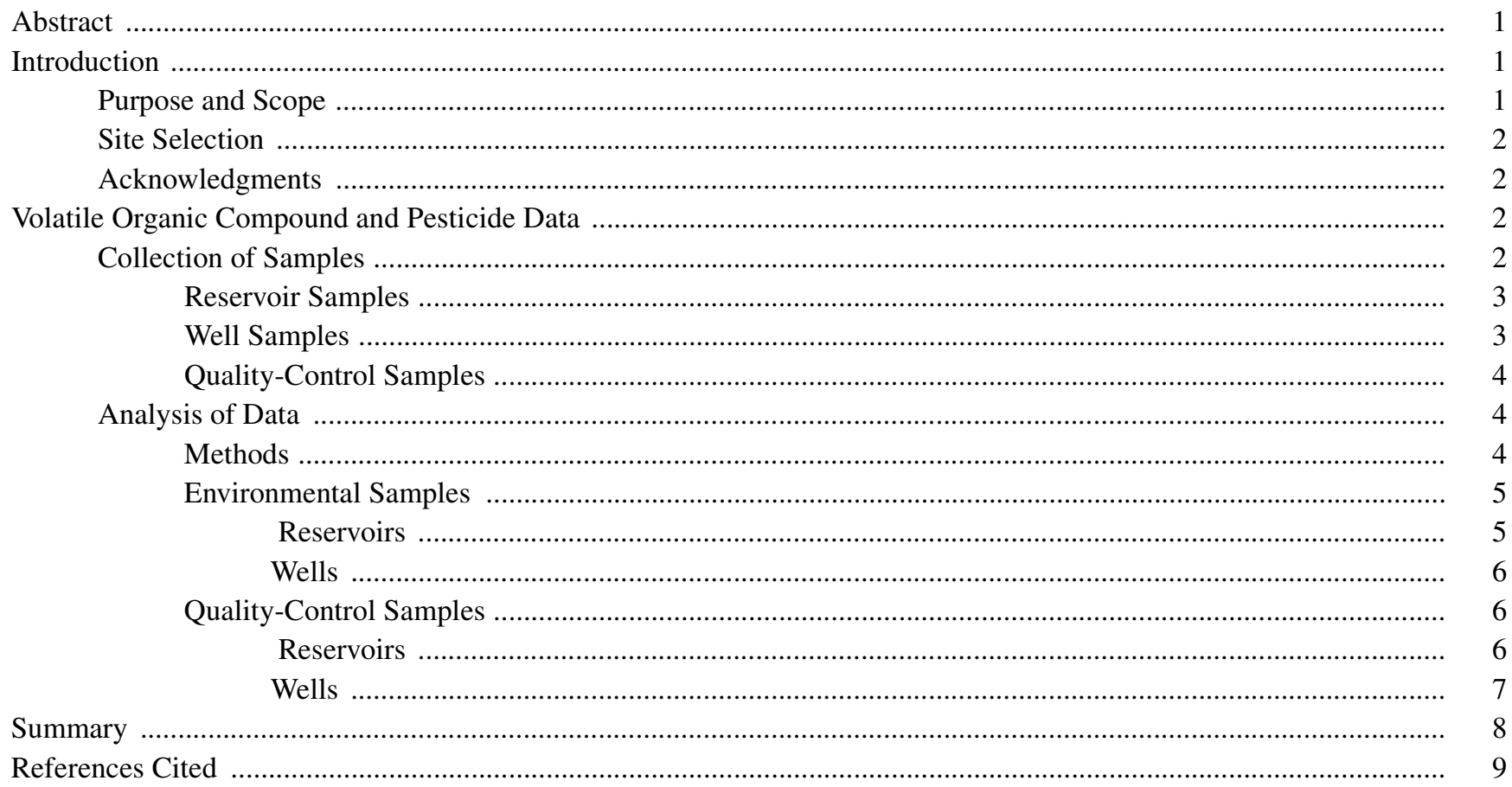

\section{FIGURES}

1. Map showing locations of public water-supply reservoirs sampled in Texas, June-July 1999 ........................ 2

2. Map showing locations of public water-supply wells sampled in Texas, November 1999-January 2001 .......... 3

3. Boxplots showing percent recovery of spikes and surrogates for reservoir samples ........................................ 6

4. Graph showing comparison of concentrations of pesticides detected in environmental and replicate

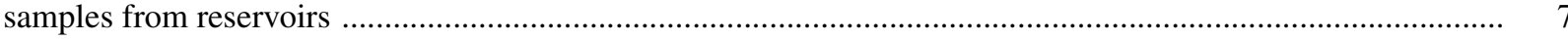

5. Boxplots showing percent recovery of spikes and surrogates for well samples ................................................ 7

6. Graph showing comparison of concentrations of pesticides detected in environmental and replicate samples from wells

(




\title{
Volatile Organic Compound and Pesticide Data for Public Water-Supply Reservoirs and Wells, Texas, 1999-2001
}

\author{
By B.J. Mahler, M.O. Gary, M.G. Canova, Eric W. Strom, Lynne Fahlquist, and \\ Michael E. Dorsey
}

\section{Abstract}

To provide data for the Texas Source-Water Assessment and Protection Program, the U.S. Geological Survey conducted a synoptic survey of 48 public water-supply reservoirs and 174 public water-supply wells during 1999-2001. The surface-water samples were analyzed for volatile organic compounds and soluble pesticides. The ground-water samples were analyzed for volatile organic compounds and soluble pesticides, as well as nitrite plus nitrate nitrogen and tritium.

One or more volatile organic compounds were detected in 75 percent of the reservoirs and in 9 percent of the wells. Methyl tert-butyl ether was detected most frequently in reservoirs, and toluene was detected most frequently in wells. One or more pesticides were detected in 96 percent of the reservoirs and in 33 percent of the wells. Atrazine or its breakdown product deethylatrazine was the most frequently detected pesticide.

Volatile organic compounds and pesticides were not detected at concentrations exceeding the maximum contaminant level allowed in drinking water. The only constituent sampled for that exceeded its maximum contaminant level (10 milligrams per liter) was nitrate nitrogen (in 8 percent of the 174 wells).

\section{INTRODUCTION}

Evaluation of the susceptibility of drinking water supplies to chemical contamination is of nationwide interest. The U.S. Environmental Protection Agency (USEPA), through the Safe Drinking Water Act Amendments of 1996, therefore required that each state complete a Source-Water Assessment and Protection (SWAP) Program. The SWAPs are to include identifica- tion of substantial potential contamination sources and to compile information regarding the susceptibility of systems to contamination. This information will, in turn, help identify systems that might be eligible for reduced monitoring or that might require additional monitoring. Assessment of susceptibility to contamination would enable more efficient use of monitoring and protection dollars.

The assessment of the susceptibility of water supplies to contamination requires accurate, consistent, and technically defensible data on the occurrence of volatile organic compounds (VOCs) and pesticides at low detection levels in source waters for public water supply (PWS). These data are needed to determine what factors or activities might contribute to contamination, which source waters are most vulnerable to contamination, and where, and for which pollutants, monitoring should be intensified or reduced. The Texas Natural Resource Conservation Commission therefore requested the U.S. Geological Survey (USGS) to collect water samples from PWS reservoirs and wells in Texas for VOC and pesticide analysis. The database of VOC and pesticide detections in PWS reservoirs and wells presented in this report represents a critical contribution to the Texas SWAP nonpoint-source pollution component for both ground-water and surface-water supplies.

\section{Purpose and Scope}

The purpose of this report is to present chemical data from the analysis of samples from surface and ground waters that are sources for PWS in Texas. Fortyeight reservoirs across Texas that are PWS source waters were sampled during June-July 1999 (fig. 1). The surface-water samples were analyzed for VOCs and a suite of soluble pesticides. One hundred and seventyfour wells that are PWS source waters were sampled during November 1999-January 2001 (fig. 2). The ground-water samples were analyzed for VOCs, a suite of soluble pesticides, nitrite plus nitrate nitrogen, and 


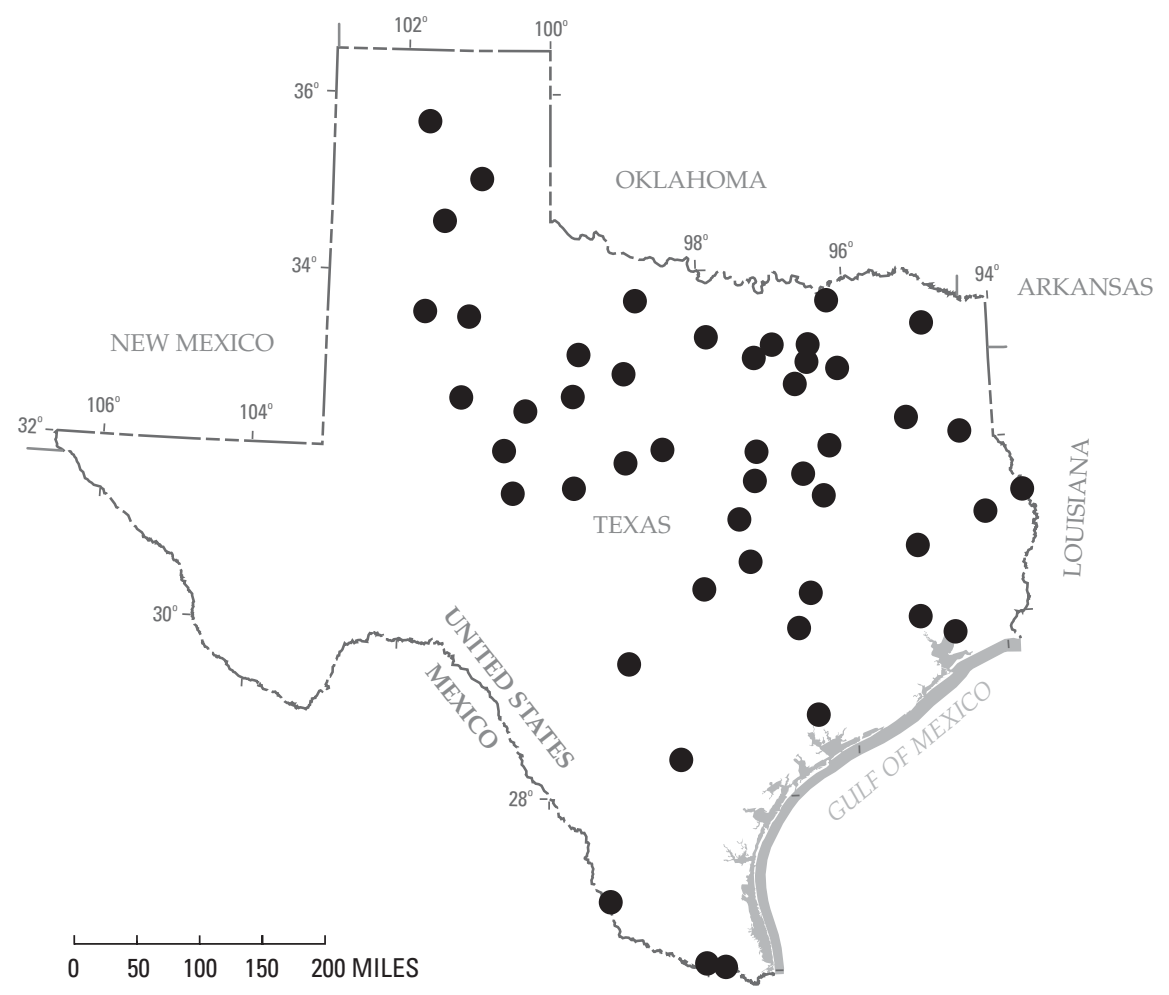

Figure 1. Locations of public water-supply reservoirs sampled in Texas, June-July 1999.

tritium. The data presented in this report will be used by the Texas SWAP to determine, to the extent possible, statistical relations between explanatory variables such as land-use characteristics of the contributing area of the supply and occurrence of a contaminant.

\section{Site Selection}

Only reservoirs that are a PWS for one or more utilities and that have a volume of 5,000 acre-feet or more were considered for sampling. The only exceptions were two small reservoirs in south Texas, La Feria Reservoir and Donna Reservoir. Of the 205 candidate reservoirs, 48 were selected to give as much spatial coverage of the State as possible and to reflect both urban and agricultural land uses in their watersheds.

The wells sampled are PWS wells that have well$\log$ information and that are screened primarily in unconfined aquifers. About 470 of about 18,000 PWS wells in Texas were candidates for sampling. From those 470 wells, 174 were selected to represent, in relative proportion, the variety of aquifer materials found in the State (for example, unconsolidated sediment, limestone, and alluvium) and to give as much spatial coverage of the State as possible. Additionally, the wells were chosen to represent a broad range of environmental characteristics such as land use, depth to water, amount of precipitation, and soil characteristics. These environmental characteristics are potential explanatory variables that might be statistically related to the occurrence of one or more contaminants.

\section{Acknowledgments}

The authors acknowledge the cooperation of the PWS well managers and operators who allowed samples to be collected during this investigation.

\section{VOLATILE ORGANIC COMPOUND AND PESTICIDE DATA}

\section{Collection of Samples}

Samples were collected following protocols established by the USGS National Water-Quality Assessment (NAWQA) Program or developed in consultation with NAWQA water-quality specialists. These sampling protocols are briefly described below and the appropriate reference cited. 


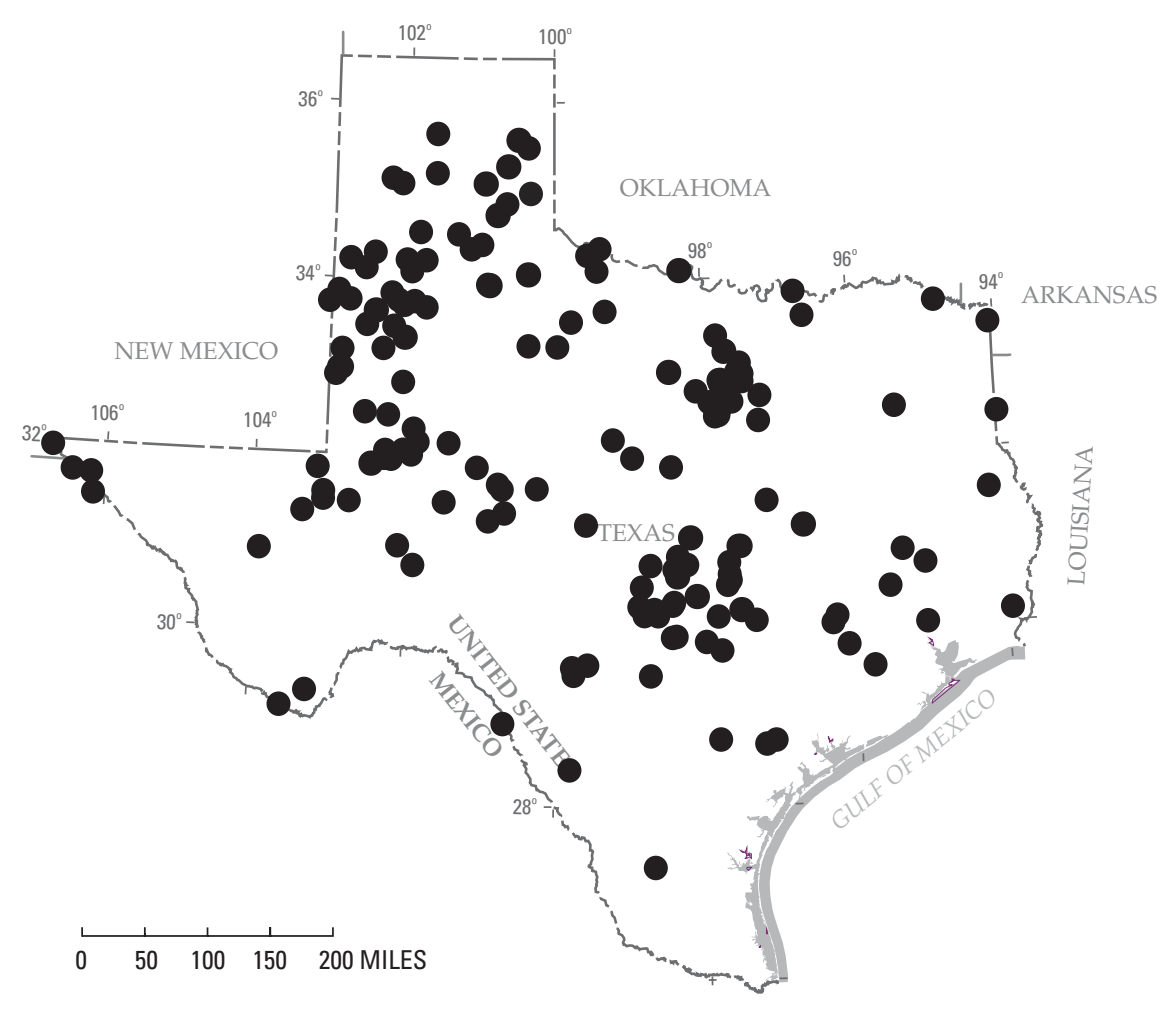

Figure 2. Locations of public water-supply wells sampled in Texas, November 1999-January 2001.

\section{Reservoir Samples}

The VOC sample was collected first at each reservoir site. Once the approximate sampling site location was reached, the boat motor was turned off and the boat allowed to drift upwind as far as possible (at least 50 feet [ft]) before the sample was collected to avoid contamination by boat exhaust. The sample was collected by dipping three pre-cleaned 40-milliliter $(\mathrm{mL})$ amber glass VOC vials about $0.5 \mathrm{~m}$ below the water surface and allowing them to fill. The samples were preserved on ice after being acidified with hydrochloric acid, agitated, and checked to make sure no air bubbles were trapped inside (Shelton, 1997).

The pesticide sample was collected as a vertical composite. The total depth at the sampling site was divided into 10 discrete depth intervals, and one-tenth of the 3-liter (L) sample was pumped from the center of each interval into a Teflon bottle using a peristaltic pump fitted with Teflon tubing. The minimum sampling interval was $2 \mathrm{ft}$, so in shallow lakes (less than $20-\mathrm{ft}$ deep), fewer than 10 intervals were sampled. The collected water was processed following NAWQA protocol (Shelton, 1994, p. 23). Briefly, the approach was to filter
1 L into each of two 1-L baked glass amber bottles using a baked 0.7-micrometer $(\mu \mathrm{m})$ pore-size glass-fiber filter in a stainless steel or aluminum holder and Teflon tubing. The samples were preserved on ice until and during shipping to the laboratory.

Dissolved oxygen, $\mathrm{pH}$, specific conductance, and temperature were measured as a vertical profile in each reservoir concurrent with collection of each pesticide sample. Measurements were taken at each of the sampling intervals using a Hydrolab and flow cell. Measurements were made by pumping lake water from the interval through the flow cell on the boat and using measurement probes inserted into the cell.

\section{Well Samples}

All well samples collected were raw water samples, that is, they were collected before the water had been filtered, chlorinated, or treated in any way. Sampling procedures followed those of the USGS NAWQA Program as described in Koterba and others (1995) and Koterba (1998).

Field properties were monitored using a Hydrolab and flow cell. The well was purged until dissolved 
oxygen, $\mathrm{pH}$, specific conductance, and temperature stabilized. Samples were not collected until the purging was completed. Samples were collected directly from the well discharge, ensuring that the water had not undergone any sort of treatment (for example, filtration or chlorination) prior to collection.

VOC vials were filled from a cleaned brass connection screwed to the well spigot. The purpose of the brass connection was to protect the sample from contact with the atmosphere. Hydrochloric acid was immediately added to each vial to lower the $\mathrm{pH}$ to $<2.0$, as a preservative. Vials were agitated and checked to make sure no air bubbles were trapped inside, then stored on ice.

Samples for pesticide analysis were either filtered in-line, when possible, or collected in a 3-L Teflon bottle and filtered off-site. In either case, water was filtered into two 1-L baked glass amber bottles using a baked $0.7-\mu \mathrm{m}$ pore-size glass-fiber filter in a stainless steel or aluminum filter apparatus. Samples were chilled on ice and returned to the USGS laboratory in Austin, Tex., for solid-phase extraction.

The nitrite plus nitrate sample was collected by repeatedly filling a $40-\mathrm{mL}$ syringe fitted with a polypropylene filter holder and filtering the sample through a $0.5-\mu \mathrm{m}$ pore-size cellulose-acetate filter into a $125-\mathrm{mL}$ high-density polyethylene (HDPE) bottle. Nitrite plus nitrate samples were stored on ice for shipping to the laboratory. The 1-L tritium sample was collected in a HDPE bottle; no filtering or treatment was required for this sample.

\section{Quality-Control Samples}

Quality-control samples for this study included trip blanks (for VOCs in reservoirs only), field blanks, replicate samples, and spiked samples. The number of quality-control samples collected is about 20 percent of the number of environmental samples collected.

\section{Analysis of Data}

The VOCs analyzed were methyl tert-butyl ether (MTBE, a gasoline oxygenate) and the BTEX compounds (benzene, toluene, ethylbenzene, and total, $o-$, and $m$ - and $p$-xylene). These compounds were chosen because there is currently much interest in the occurrence of MTBE in the environment and because the BTEX compounds are among the more commonly occurring VOCs in surface and ground water (Grady and Casey, 2000). The BTEX compounds are among the constituents regulated by the Safe Drinking Water Act.

The pesticides analyzed include many of those regulated by the Safe Drinking Water Act, as well as others that are under consideration for regulation. Most of these pesticides are very soluble in water thus more likely to occur in source waters. Most of these pesticides also are widely used and include the herbicides atrazine and 2,4-D and the insecticides carbaryl and dieldrin.

In this report, nitrate refers to the sum of nitrite plus nitrate nitrogen, which consists primarily of nitrate with negligible amounts of nitrite. Nitrite plus nitrate nitrogen was analyzed in the samples from 174 PWS wells because the presence of nitrate could be an indicator of other types of contamination. For example, nitrate is commonly used in fertilizer, and its occurrence in high concentrations in drinking water might indicate contamination by agricultural runoff. In addition, concentrations of nitrate exceed the U.S. Environmental Protection Agency (1996) maximum contaminant level (MCL) of 10 milligrams per liter $(\mathrm{mg} / \mathrm{L})$ in drinking water relatively often (U.S. Geological Survey, 2001).

Tritium was analyzed in ground water as an indication of the age of the water (Schlosser and others, 1988). Tritium is a radionuclide produced naturally in the outer atmosphere. It is also produced by nuclear reactors and nuclear-weapons testing. It is incorporated into the water molecule and has a half-life of 12.3 years, two characteristics that make it useful as an indicator of ground-water age, particularly for differentiating between older (pre-1953) and younger (post-1953) water. The analysis of tritium was included in this study to assist the SWAP in determining if the occurrence of tritium, a sign of relatively young waters, might be a useful indicator of contamination.

\section{Methods}

The VOCs were analyzed at the USGS National Water Quality Laboratory (NWQL) in Denver, Colo., by gas chromatograph/mass spectrometry (GC/MS) at a minimum reporting level (MRL) of 0.2 microgram per liter $(\mu \mathrm{g} / \mathrm{L})$. The procedures are described by Connor and others (1998). Samples for pesticide analysis were first extracted onto solid-phase columns at the USGS laboratory in Austin, then submitted to the NWQL for analysis by GC/MS. Pesticide analytical procedures are described by Zaugg and others (1995) and Werner and others (1996). Nitrite plus nitrate was analyzed at the NWQL by colorimetry and cadmium reduction and by 
diazotization, as described by Fishman (1993), at an MRL of $0.05 \mathrm{mg} / \mathrm{L}$. Tritium was analyzed at the USGS National Research Program Laboratory in Menlo Park, Calif. Tritium analysis was done by electrolytic enrichment and liquid scintillation (Thatcher and others, 1977) at an MRL of 2.5 picocuries per liter (pCi/L).

Analytical results are listed in tables 1-6 (at end of report). Concentrations are reported as less-than values, estimated values, or actual sample values. Lessthan values are reported when the constituent was not detected above a specified concentration. Less-than values are based on the MRL, defined as the lowest concentration of a constituent that can be identified and quantitated within known statistical limits. The MRL could be raised as a result of high concentrations of target or non-target constituents. Estimated values are concentrations that are less than the MRL but greater than the laboratory method detection limit. These concentrations are identified as estimated values because of the uncertainty associated with concentrations less than the MRL. Estimated values thus are counted as a detection, but quantification of the concentration is uncertain as estimated values are typically outside of the calibration range of the method.

\section{Environmental Samples}

\section{Reservoirs}

One or more VOCs were detected in 36 of the 48 PWS reservoirs (75 percent) during the June-July 1999 sampling (table 1). MTBE was detected in 34 of the 48 reservoirs; all concentrations were well below the USEPA drinking-water advisory level of 20 to $40 \mu \mathrm{g} / \mathrm{L}$ issued for reasons of taste and smell (U.S. Environmental Protection Agency, 1997). Eleven reservoirs had MTBE concentrations exceeding $1.0 \mu \mathrm{g} / \mathrm{L}$. The highest concentration of MTBE detected during this investigation was $4.1 \mu \mathrm{g} / \mathrm{L}$ in a sample from Grapevine Lake.

To investigate the influence of recreational use of gasoline-powered vehicles on MTBE concentrations, four reservoirs with high recreational use (Granger Lake, Lake Belton, Lake Travis, and Lake Waco) were sampled before and after the Fourth of July weekend. In each case, the MTBE concentration after the high-use weekend was greater than that analyzed previously. On the basis of the Sign Test for matched pairs (Helsel and Hirsch, 1992), the difference is significant at $p=0.06$.

Occurrence of the BTEX compounds was less widespread than that of MTBE. Benzene was detected in 6 reservoirs, toluene in 19 reservoirs, ethylbenzene in
6 reservoirs, and xylenes in 11 reservoirs. The highest concentrations of BTEX compounds were $0.75 \mu \mathrm{g} / \mathrm{L}$ of benzene, $2.9 \mu \mathrm{g} / \mathrm{L}$ of toluene, $0.44 \mu \mathrm{g} / \mathrm{L}$ of ethylbenzene, and $2.2 \mu \mathrm{g} / \mathrm{L}$ of total xylene in a sample from Lake Medina. These concentrations are well below the MCLs of $5.0 \mu \mathrm{g} / \mathrm{L}$ for benzene, $1,000 \mu \mathrm{g} / \mathrm{L}$ for toluene, 700 $\mu \mathrm{g} / \mathrm{L}$ for ethylbenzene, and $10,000 \mu \mathrm{g} / \mathrm{L}$ for total xylene. Concentrations of BTEX also were affected by high recreational use of lakes over the Fourth of July weekend. Toluene concentrations in three of the four reservoirs and xylene concentrations in two reservoirs were higher following the high-use weekend; in two reservoirs, benzene and ethylbenzene were not detected before the high-use weekend but were detected afterward at concentrations less than the MRL. On the basis of the Sign Test for matched pairs, these differences are not significant at alpha $=0.1$.

One or more pesticides were detected in 46 of the 48 reservoirs (96 percent) (table 2). The pesticide detections are summarized in table 3 . Of the 10 pesticides analyzed for which MCLs have been established, only 4 pesticides were detected, and concentrations did not exceed those respective MCLs. Atrazine, the most commonly detected pesticide, was detected in every reservoir except two, Lakes Murvaul and Sweetwater; no pesticides were detected in samples from those two lakes. The highest atrazine concentration was $1.4 \mu \mathrm{g} / \mathrm{L}$ from Granger Lake. The MCL for atrazine is $3.0 \mu \mathrm{g} / \mathrm{L}$. Deethylatrazine, a breakdown product of atrazine, was detected in 43 reservoirs.

The next two most commonly detected pesticides were simazine and metolachlor, detected in 29 and 28 reservoirs, respectively. The highest concentration of simazine was $0.52 \mu \mathrm{g} / \mathrm{L}$ in Lake Ray Hubbard. The MCL for simazine is $4.0 \mu \mathrm{g} / \mathrm{L}$, and the U.S. Environmental Protection Agency (1996) lifetime Health Advisory (HA) level is $1.0 \mu \mathrm{g} / \mathrm{L}$. The highest concentration of metolachlor was $0.29 \mu \mathrm{g} / \mathrm{L}$ in Aquilla Lake. The HA for metolachlor is $70 \mu \mathrm{g} / \mathrm{L}$.

The most pesticides detected in any reservoir was 12, in Lake Texana. Other reservoirs with eight or more pesticides detected were Buffalo Springs Lake, Granger Lake, Lake Houston, Lavon Lake, and Livingston Reservoir. Of the 46 reservoirs with pesticide detections, 45 reservoirs ( 94 percent) had detections of two or more pesticides, and 27 reservoirs (59 percent) had detections of five or more pesticides. 


\section{Wells}

One or more VOCs were detected in 16 of the 174 PWS wells (9 percent) during the November 1999January 2001 sampling (table 4). Toluene was the most commonly detected VOC, with detections in 13 of the 174 wells (7 percent). The other VOCs, including MTBE, were detected either one or two times each. The highest concentration of toluene was $1.2 \mu \mathrm{g} / \mathrm{L}$, about 3 orders of magnitude less than the MCL of $1,000 \mu \mathrm{g} / \mathrm{L}$. Only one VOC was detected in each of 14 wells, two VOCs were detected in 1 well, and five VOCs were detected in 1 well.

One or more pesticides were detected in 58 of the 174 wells ( 33 percent) (table 5). The pesticide detections are summarized in table 6. Deethylatrazine was the most frequently detected pesticide in ground water with detections in 38 wells ( 22 percent). The highest concentration of deethylatrazine was $1.7 \mu \mathrm{g} / \mathrm{L}$. Atrazine was the second most frequently detected pesticide, in 27 wells (16 percent). The highest concentration of atrazine was $1.5 \mu \mathrm{g} / \mathrm{L}$. Prometon, detected in 22 wells (13 percent), was the third most frequently detected pesticide. The highest concentration of prometon was $0.12 \mu \mathrm{g} / \mathrm{L}$. All other pesticides were detected in less than 10 wells. Of the 10 pesticides analyzed for which MCLs have been established, only 4 pesticides were detected, and concentrations did not exceed those respective MCLs. The most pesticides detected in any single well was six - atrazine, deethylatrazine, diuron, prometon, simazine, and tebuthiuron in a well (ZT-27-02-105) in Denver City, Tex. Thirtyeight of the wells had detections of two or more pesticides, and five wells had detections of five or more pesticides.

Nitrate concentrations (table 4) exceeded the MCL of $10 \mathrm{mg} / \mathrm{L}$ in 14 of the 174 wells (8 percent). The highest concentration detected was $23 \mathrm{mg} / \mathrm{L}$ in a well (ZH-13-61-620) in Wichita Falls, Tex. Nitrate was the only constituent in this study that exceeded the MCL.

Tritium, a radioactive isotope of hydrogen, was analyzed in the ground-water samples to determine if its presence was related to the occurrence of pesticides. Of the 168 PWS wells analyzed for tritium (table 4), 58 wells had tritium concentrations less than the method detection limit. Pesticides were detected in nine of those wells (16 percent). In contrast, pesticides were detected in 47 of the 110 wells in which tritium was detected (43 percent).

\section{Quality-Control Samples}

\section{Reservoirs}

No VOCs were detected in the three reservoir trip blanks. One VOC, toluene, was detected in one of four reservoir field blanks collected; however, toluene was not detected in other environmental samples collected during that sampling trip. Nondetections in the three replicate samples corresponded either to nondetections or to detection below the MRL in parallel environmental samples. In the two cases where VOCs were detected in both the replicate sample and the corresponding environmental sample, the difference was less than 10 percent. Recovery of VOC spiked samples ranged from 42 to 152 percent, and 95 percent of VOC surrogate recoveries were within acceptable levels as set by the NWQL (fig. 3).

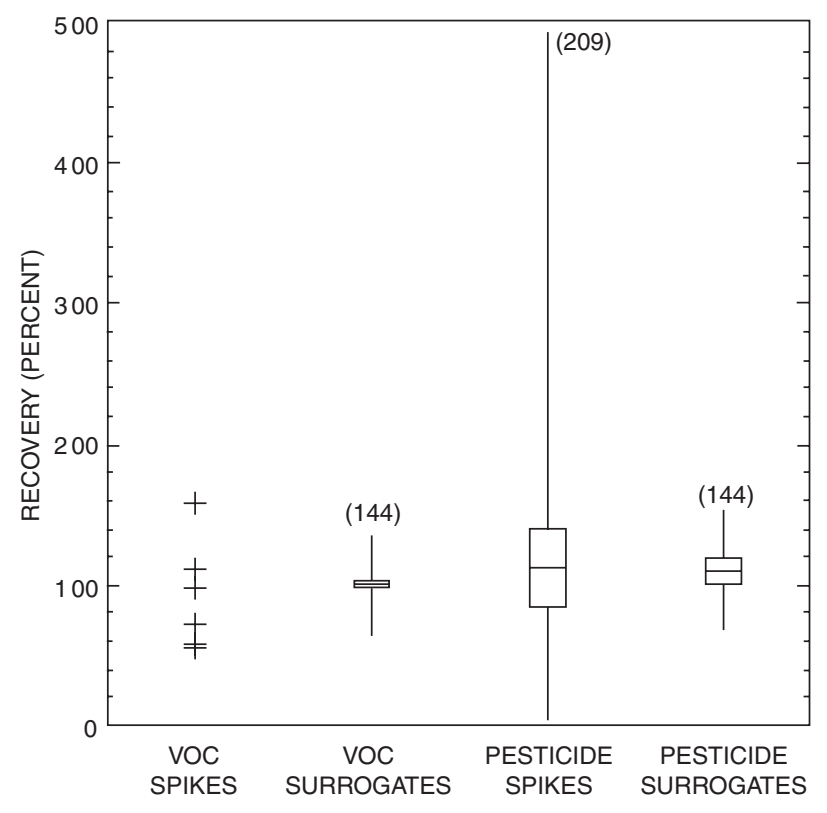

\section{EXPLANATION}

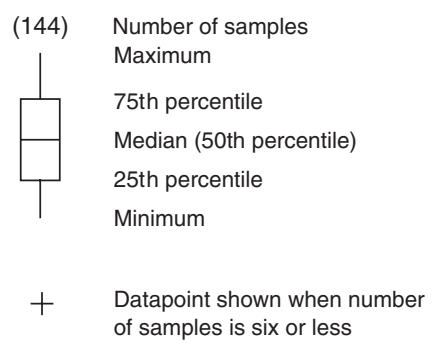

Figure 3. Percent recovery of spikes and surrogates for reservoir samples. 
No pesticides were detected in the four reservoir field blanks. Nondetections of pesticides in the three replicate samples corresponded in all cases to nondetections in the parallel environmental sample. When pesticides were detected in both the replicate sample and the corresponding environmental sample, the difference was within 10 percent with two exceptions of 12 percent (fig. 4). Recovery in spiked samples ranged from 0 to 484 percent, with 50 percent of the recoveries between 87 and 140 percent (fig. 3); 87 percent of pesticide surrogate recoveries were within acceptable levels as set by the NWQL (fig. 3).

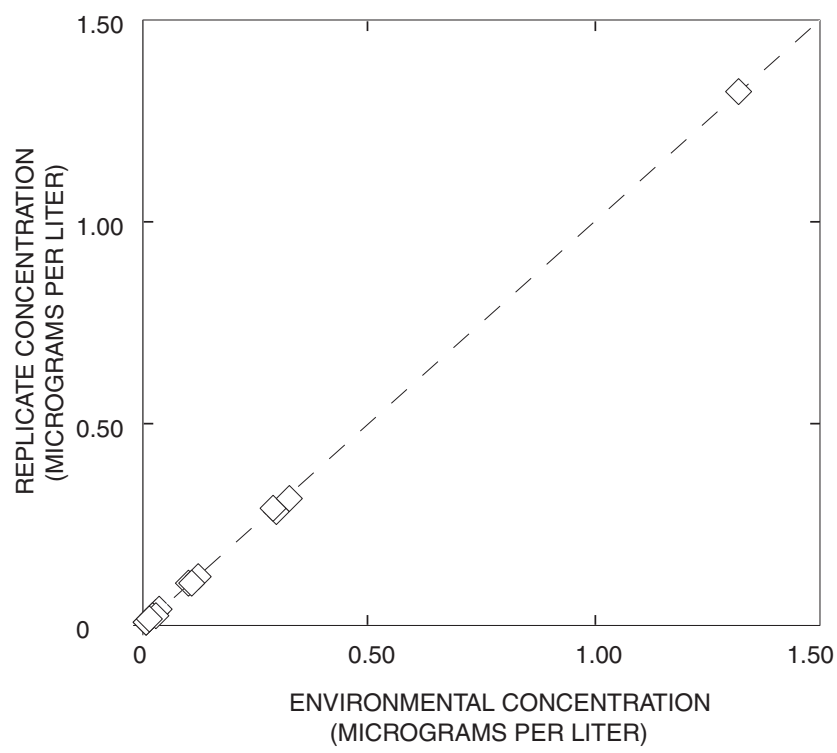

Figure 4. Comparison of concentrations of pesticides detected in environmental and replicate samples from reservoirs.

\section{Wells}

No VOCs were detected in the nine ground-water field blanks, except toluene, which was detected in one field blank at a concentration less than the MRL of $0.2 \mu \mathrm{g} / \mathrm{L}$. However, toluene was not detected in any environmental samples collected during that sampling trip. Nondetections of VOCs in the seven replicate samples corresponded to nondetections in the parallel environmental samples. In the one case where a VOC was detected in both the replicate sample and the corresponding environmental sample, the difference was 15 percent. Recovery in spiked samples ranged from 73 to 157 percent, with 50 percent of the recoveries between 77 and 108 percent (fig. 5); 94 percent of VOC surrogate recoveries were within acceptable levels, as set by the NWQL (fig. 5).

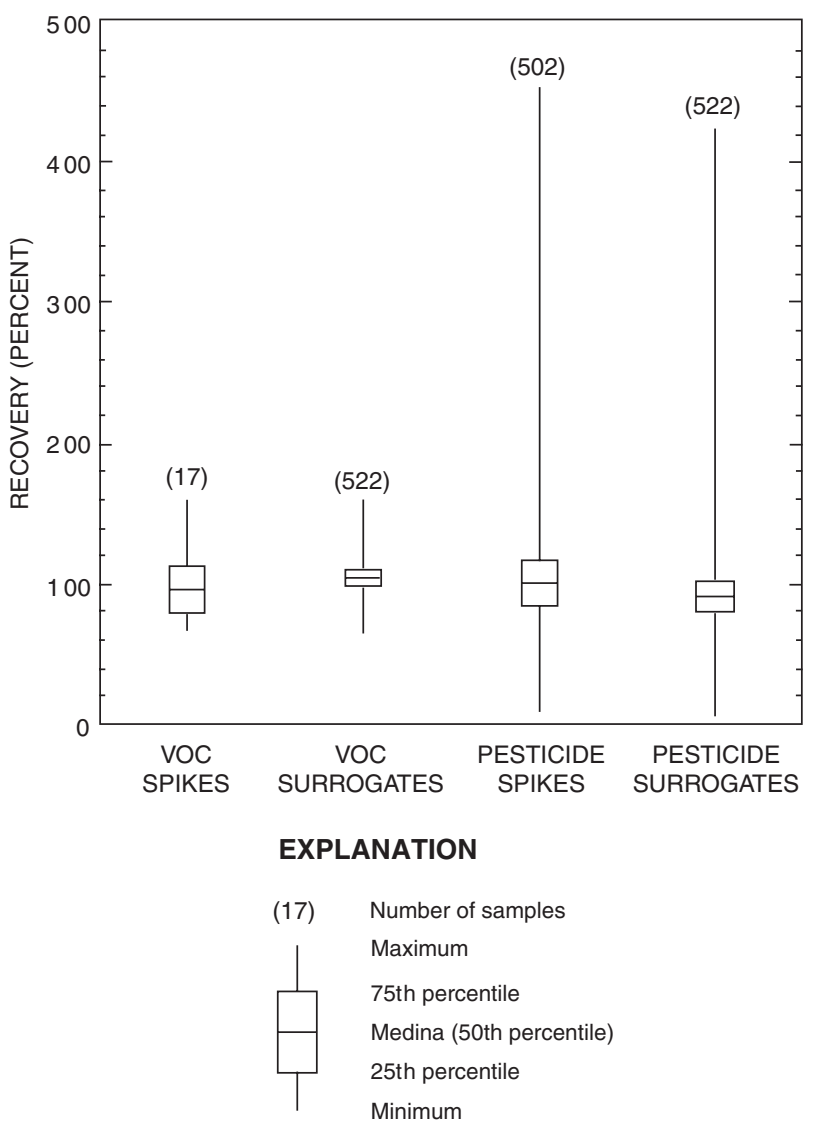

Figure 5. Percent recovery of spikes and surrogates for well samples.

One pesticide, diazinon, was detected in 2 of the 12 blanks at concentrations about 3 and 6 times the MRL of $0.002 \mu \mathrm{g} / \mathrm{L}$. Diazinon was not detected in any environmental samples collected during those sampling trips. Nondetections of pesticides in the seven replicate samples corresponded to nondetections in the parallel environmental samples. When pesticides were detected in both the replicate sample and the corresponding environmental sample, the difference ranged from 2.4 to 18 percent (fig. 6). Recovery in spiked samples ranged from 9 to 441 percent, with 50 percent of the recoveries between 84 and 114 percent (fig. 5); 98 percent of pesticide surrogate recoveries were within acceptable levels, as set by the NWQL (fig. 5). 


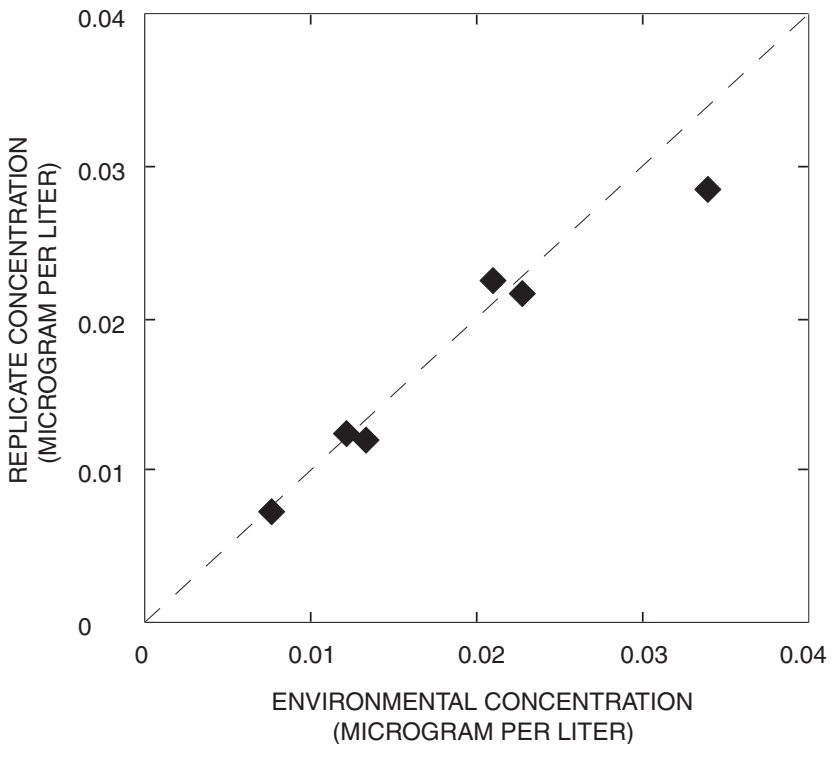

Figure 6. Comparison of concentrations of pesticides detected in environmental and replicate samples from wells.

\section{SUMMARY}

During 1999-2001 the USGS collected waterquality samples from 48 PWS reservoirs and 174 PWS wells in Texas at the request of the TNRCC to provide data for the Texas SWAP Program. Samples from the reservoirs were analyzed for VOCs and a suite of soluble pesticides. Samples from the wells were analyzed for VOCs, a suite of soluble pesticides, nitrite plus nitrate nitrogen, and tritium.

One or more VOCs were detected in 36 of the 48 reservoirs ( 75 percent). MTBE was the most frequently detected VOC with detections in 34 of the 48 reservoirs. The highest concentration was $4.1 \mu \mathrm{g} / \mathrm{L}$; all concentrations were well below the USEPA drinking-water advisory level of 20 to $40 \mu \mathrm{g} / \mathrm{L}$. Four reservoirs with high recreational use were sampled before and after the 1999 Fourth of July weekend to investigate the influence of recreational use of gasoline-powered vehicles on MTBE concentrations. The MTBE concentration in each of the four reservoirs was higher after the Fourth of July weekend than before. The BTEX compounds were detected less frequently than MTBE; all concentrations were well below the respective MCLs established by the USEPA. Benzene was detected in 6 reservoirs (highest concentration $0.75 \mu \mathrm{g} / \mathrm{L}$ ), toluene was detected in 19 reservoirs (highest concentration $2.9 \mu \mathrm{g} / \mathrm{L}$ ), ethylbenzene was detected in 6 reservoirs (highest concentration $0.44 \mu \mathrm{g} / \mathrm{L}$ ), and total xylene was detected in 11 reservoirs (highest concentration $2.2 \mu \mathrm{g} / \mathrm{L}$ ).

All but two reservoirs had detections of one or more pesticides (96 percent). Atrazine was the most frequently detected pesticide with detections in 46 of the 48 reservoirs (96 percent). The highest concentration, $1.4 \mu \mathrm{g} / \mathrm{L}$, was less than the MCL of $3.0 \mu \mathrm{g} / \mathrm{L}$. Deethylatrazine, a breakdown product of atrazine, was detected in 43 reservoirs (90 percent). Simazine was detected in 29 reservoirs (60 percent) with a maximum concentration of $0.52 \mu \mathrm{g} / \mathrm{L}$; all concentrations were less than the MCL of $4.0 \mu \mathrm{g} / \mathrm{L}$ and the USEPA HA level of $1.0 \mu \mathrm{g} / \mathrm{L}$. Metolachlor was detected in 28 reservoirs (58 percent) with a maximum concentration of $0.29 \mu \mathrm{g} / \mathrm{L}$; all concentrations were less than the HA of $70 \mu \mathrm{g} / \mathrm{L}$. Fifteen other pesticides also were detected at frequencies ranging from 40 to 2.1 percent. The most pesticides detected in any reservoir was 12 .

One or more VOCs were detected in 16 of the 174 wells ( 9 percent). Toluene was the most frequently detected VOC with detections in 13 of the 174 wells (7 percent). The highest concentration of toluene was $1.2 \mu \mathrm{g} / \mathrm{L}$, much less than the MCL of $1,000 \mu \mathrm{g} / \mathrm{L}$. Only one VOC was detected in each of 14 wells, two VOCs were detected in 1 well, and five VOCs were detected in 1 well.

One or more pesticides were detected in 58 of the 174 wells (33 percent). Deethylatrazine, the most frequently detected pesticide with detections in 38 wells (22 percent), had a maximum concentration of $1.7 \mu \mathrm{g} / \mathrm{L}$. Atrazine was detected in 27 wells (16 percent) with a maximum concentration of $1.5 \mu \mathrm{g} / \mathrm{L}$; prometon was detected in 22 wells (13 percent) with a maximum concentration of $0.12 \mu \mathrm{g} / \mathrm{L}$. Thirteen other pesticides also were detected at frequencies ranging from 5.2 to 0.6 percent. The most pesticides detected in any well was six. Two or more pesticides were detected in 38 wells, and five or more pesticides were detected in 5 wells.

Nitrate nitrogen (highest concentration $23 \mathrm{mg} / \mathrm{L}$ ) exceeded the MCL of $10 \mathrm{mg} / \mathrm{L}$ in 14 of the 174 wells (8 percent). Nitrate was the only constituent in the study to exceed the MCL. Tritium was analyzed for in 168 wells and was less than the method detection limit in 58 wells. Pesticides were detected in 9 of the 58 wells (16 percent); in contrast, of the 110 wells in which tritium was detected, 47 wells (43 percent) also had detections of pesticides. 


\section{REFERENCES CITED}

Connor, B.F., Rose, D.L., Noriega, M.C., Murtagh, L.K., and Abney, S.R., 1998, Methods of analysis by the U.S. Geological Survey National Water Quality LaboratoryDetermination of 86 volatile organic compounds in water by gas chromatography/mass spectrometry, including detections less than reporting limits: U.S. Geological Survey Open-File Report 97-829, 78 p.

Fishman, M.J., 1993, Methods of analysis by the U.S. Geological Survey National Water Quality LaboratoryDetermination of inorganic and organic constituents in water and fluvial sediments: U.S. Geological Survey Open-File Report 93-125, 217 p.

Grady, S.J., and Casey, G.D., 2000, Occurrence and distribution of methyl tert-butyl ether and other volatile organic compounds in drinking water in the northeast and midAtlantic regions of the United States, 1993-98: U.S. Geological Survey Water-Resources Investigations Report 00-4228, 123 p.

Helsel, D.R., and Hirsch, R.M., 1992, Studies in environmental science 49-Statistical methods in water resources: Amsterdam, Elsevier, 529 p.

Koterba, M.T., 1998, Ground-water data-collection protocols and procedures for the National Water-Quality Assessment Program-Collection, documentation, and compilation of required site, well, subsurface, and landscape data for wells: U.S. Geological Survey Open-File Report 98-4107, $91 \mathrm{p}$.

Koterba, M.T., Wilde, F.D., and Lapham, W.W., 1995, Ground-water data-collection protocols and procedures for the National Water-Quality Assessment ProgramCollection and documentation of water-quality samples and related data: U.S. Geological Survey Open-File Report 95-399, 113 p.

Schlosser, P., Stute, M., Dörr, H., Sonntag, C., and Münnich, K.O., 1988, Tritium/3He dating of shallow groundwater: Earth and Planetary Science Letters, v. 89, p. 353-362.
Shelton, L.R., 1994, Field guide for collecting and processing stream-water samples for the National Water-Quality Assessment Program: U.S. Geological Survey OpenFile Report 94-455, 50 p.

1997, Field guide for collecting samples for analysis of volatile organic compounds in stream water for the National Water-Quality Assessment Program: U.S. Geological Survey Open-File Report 97-401, 14 p.

Thatcher, L.L., Janzer, V.J., and Edwards, K.W., 1977, Methods for determination of radioactive substances in water and fluvial sediments: U.S. Geological Survey Techniques of Water-Resources Investigations, book 5, chap. A5, $95 \mathrm{p}$.

U.S. Environmental Protection Agency, 1996, Drinking water regulations and health advisories: U.S. Environmental Protection Agency, Office of Water, EPA 822-B-96002, 16 p.

1997, Drinking-water advisory-Consumer acceptability advice and health effects analysis on methyl tertiary-butyl ether (MTBE):U.S. Environmental Protection Agency, Office of Water, EPA 822-F-97-008.

U.S. Geological Survey, 2001, Selected findings and current perspectives on urban and agricultural water quality by the National Water Quality Assessment Program: U.S. Geological Survey Fact Sheet FS-047-01, 2 p.

Werner, S.L., Burkhardt, M.R., and DeRusseau, S.N., 1996, Methods of analysis by the U.S. Geological Survey National Water Quality Laboratory-Determination of pesticides in water by Carbopak-B solid-phase extraction and high-performance liquid chromatography: U.S. Geological Survey Open-File Report 96-216, $42 \mathrm{p}$.

Zaugg, S.D., Sandstrom, M.W., Smith, S.G., and Fehlberg, K.M., 1995, Methods of analysis by the U.S. Geological Survey National Water Quality Laboratory-Determination of pesticides in water by $\mathrm{C}-18$ solid-phase extraction and capillary-column gas chromatography/mass spectrometry with selected ion monitoring: U.S. Geological Survey Open-File Report 95-181, 49 p. 
Table 1. Concentrations of volatile organic compounds in public water-supply reservoirs sampled in Texas, June-July 1999

$[\mu \mathrm{g} / \mathrm{L}$, micrograms per liter; <, less than; E, estimated]

\begin{tabular}{|c|c|c|c|c|c|c|c|c|c|c|}
\hline Site name & $\begin{array}{c}\text { Site } \\
\text { identifier }\end{array}$ & $\begin{array}{l}\text { Station } \\
\text { number }\end{array}$ & Date & $\begin{array}{l}\text { MTBE } \\
(\mu \mathrm{g} / \mathrm{L})\end{array}$ & $\begin{array}{c}\text { Benzene } \\
(\mu \mathrm{g} / \mathrm{L})\end{array}$ & $\begin{array}{l}\text { Toluene } \\
(\mu \mathrm{g} / \mathrm{L})\end{array}$ & $\begin{array}{l}\text { Ethyl- } \\
\text { ben- } \\
\text { zene } \\
(\mu \mathrm{g} / \mathrm{L})\end{array}$ & $\begin{array}{c}\text { Total } \\
\text { xylene } \\
(\mu \mathrm{g} / \mathrm{L})\end{array}$ & $\begin{array}{c}o- \\
\text { Xylene } \\
(\mu \mathrm{g} / \mathrm{L})\end{array}$ & $\begin{array}{c}m \text { - and } p- \\
\text { Xylene } \\
(\mu \mathrm{g} / \mathrm{L})\end{array}$ \\
\hline Buffalo Springs Lake & PS & 333205101414401 & $07-12-99$ & 1.1 & $<.4$ & .30 & $<.4$ & $<.4$ & $<.4$ & $<.4$ \\
\hline Cedar Creek Reservoir & PS & 295502096440201 & 07-19-99 & .72 & $<.2$ & .20 & $<.2$ & .22 & $<.2$ & .22 \\
\hline Choke Canyon Reservoir & $\mathrm{AC}$ & 8206890 & 07-07-99 & $<.2$ & $<.2$ & $<.2$ & $<.2$ & $<.2$ & $<.2$ & $<.2$ \\
\hline E.V. Spence Reservoir & PS & 315535100341701 & 07-15-99 & $<.4$ & $<.4$ & $<.4$ & $<.4$ & $<.4$ & $<.4$ & $<.4$ \\
\hline Falcon International Reservoir & $\mathrm{BC}$ & 263815099111901 & 07-07-99 & $<.2$ & $<.2$ & $<.2$ & $<.2$ & $<.2$ & $<.2$ & $<.2$ \\
\hline Fort Phantom Hill Reservoir & PS & 323632099410401 & 07-14-99 & E.11 & $<.2$ & .20 & $<.2$ & $<.2$ & $<.2$ & $<.2$ \\
\hline Granger Lake & $\mathrm{AC}$ & 304132097200801 & 06-30-99 & E. 10 & $<.2$ & $<.2$ & $<.2$ & $<.2$ & $<.2$ & $<.2$ \\
\hline Granger Lake & $\mathrm{AC}$ & 304132097200801 & 07-06-99 & .37 & $<.2$ & $<.2$ & $<.2$ & $<.2$ & $<.2$ & $<.2$ \\
\hline Lake Anahuac & PS & 294624094411201 & $07-21-99$ & .26 & $<.2$ & $<.2$ & $<.2$ & $<.2$ & $<.2$ & $<.2$ \\
\hline Lake Belton & $\mathrm{BC}$ & 310711097302201 & 06-30-99 & .53 & $<.2$ & $<.2$ & $<.2$ & $<.2$ & $<.2$ & $<.2$ \\
\hline Lake Belton & $\mathrm{BC}$ & 310711097302201 & 07-07-99 & .80 & $<.2$ & .37 & .13 & .75 & .23 & .52 \\
\hline Lake Bonham & PS & 333858096083301 & $07-27-99$ & 1.0 & $<.2$ & .12 & $<.2$ & $<.2$ & $<.2$ & $<.2$ \\
\hline Lake Bridgeport & Mid-lake & 331312097501801 & $07-21-99$ & .25 & $<.2$ & $<.2$ & $<.2$ & $<.2$ & $<.2$ & $<.2$ \\
\hline Lake Brownwood & PS & 315026099005301 & 07-19-99 & .99 & $<.2$ & .27 & $<.2$ & $<.2$ & $<.2$ & $<.2$ \\
\hline Lake Houston & $\mathrm{BC}$ & 295702095091401 & $07-12-99$ & 3.0 & $<.2$ & $<.2$ & $<.2$ & $<.2$ & $<.2$ & $<.2$ \\
\hline Lake J.B. Thomas & PS & 323510101085501 & 07-13-99 & $<.2$ & $<.2$ & .26 & $<.2$ & $<.2$ & $<.2$ & $<.2$ \\
\hline Lake Kickapoo & PS & 333913098480601 & 07-19-99 & $<.2$ & $<.2$ & $<.2$ & $<.2$ & $<.2$ & $<.2$ & $<.2$ \\
\hline Lake Limestone & $\mathrm{BC}$ & 312458096205101 & 07-08-99 & .43 & $<.2$ & .14 & $<.2$ & $<.2$ & $<.2$ & $<.2$ \\
\hline Lake Meredith & $\mathrm{BC}$ & 354113101360101 & 07-13-99 & $<.2$ & $<.2$ & $<.2$ & $<.2$ & $<.2$ & $<.2$ & $<.2$ \\
\hline Lake Mexia & PS & 313848096345001 & 07-07-99 & 1.2 & $<.2$ & $<.2$ & $<.2$ & $<.2$ & $<.2$ & $<.2$ \\
\hline
\end{tabular}


Table 1. Concentrations of volatile organic compounds in public water-supply reservoirs sampled in Texas, June-July 1999—Continued

\begin{tabular}{|c|c|c|c|c|c|c|c|c|c|c|}
\hline Site name & $\begin{array}{c}\text { Site } \\
\text { identifier }\end{array}$ & $\begin{array}{l}\text { Station } \\
\text { number }\end{array}$ & Date & $\begin{array}{l}\text { MTBE } \\
(\mu \mathrm{g} / \mathrm{L})\end{array}$ & $\begin{array}{c}\text { Benzene } \\
(\mu \mathrm{g} / \mathrm{L})\end{array}$ & $\begin{array}{l}\text { Toluene } \\
(\mu \mathrm{g} / \mathrm{L})\end{array}$ & $\begin{array}{l}\text { Ethyl- } \\
\text { ben- } \\
\text { zene } \\
(\mu \mathrm{g} / \mathrm{L})\end{array}$ & $\begin{array}{c}\text { Total } \\
\text { xylene } \\
(\mu \mathrm{g} / \mathrm{L})\end{array}$ & $\begin{array}{c}o- \\
\text { Xylene } \\
(\mu \mathrm{g} / \mathrm{L})\end{array}$ & $\begin{array}{c}m \text { - and } p- \\
\text { Xylene } \\
(\mu \mathrm{g} / \mathrm{L})\end{array}$ \\
\hline Lake Ray Hubbard & PS & 324929096305601 & 07-09-99 & .75 & $<.2$ & $<.2$ & $<.2$ & $<.2$ & $<.2$ & $<.2$ \\
\hline Lake Stamford & PS & 330414099340201 & 07-20-99 & $<.4$ & $<.4$ & $<.4$ & $<.4$ & $<.4$ & $<.4$ & $<.4$ \\
\hline Lake Sweetwater & PS & 322609100181901 & 07-14-99 & .35 & $<.2$ & $<.2$ & $<.2$ & $<.2$ & $<.2$ & $<.2$ \\
\hline Lake Texana & $\mathrm{CC}$ & 285816096320201 & 07-20-99 & E.13 & $<.2$ & $<.2$ & $<.2$ & $<.2$ & $<.2$ & $<.2$ \\
\hline Lake Travis & PS & 302429097541401 & 06-28-99 & 1.2 & $<.2$ & .28 & $<.2$ & E.12 & $<.2$ & E.12 \\
\hline Lake Travis & PS & 302429097541401 & 07-06-99 & 2.3 & .34 & .80 & $<.2$ & E.11 & $<.2$ & E.11 \\
\hline Lake Tyler & PS & 321246095101701 & 07-16-99 & $<.2$ & $<.2$ & $<.2$ & $<.2$ & $<.2$ & $<.2$ & $<.2$ \\
\hline Lake Waco & $\mathrm{AC}$ & 313430097113801 & 06-30-99 & E.15 & $<.2$ & $<.2$ & $<.2$ & $<.2$ & $<.2$ & $<.2$ \\
\hline McKenzie Reservoir & PS & 343257101271001 & 07-14-99 & E.11 & $<.2$ & $<.2$ & $<.2$ & $<.2$ & $<.2$ & $<.2$ \\
\hline Medina Lake & $\mathrm{AC}$ & 293225098560600 & 07-06-99 & 1.3 & .75 & 2.9 & .44 & 2.2 & .68 & 1.5 \\
\hline O.C. Fisher Lake & PS & 312909100293901 & $07-15-99$ & $<.4$ & $<.4$ & $<.4$ & $<.4$ & $<.4$ & $<.4$ & $<.4$ \\
\hline O.H. Ivie Reservoir & PS & 313130099391001 & 07-16-99 & $<.2$ & $<.2$ & $<.2$ & $<.2$ & $<.2$ & $<.2$ & $<.2$ \\
\hline Proctor Lake & $\mathrm{AC}$ & 315814098291201 & 07-20-99 & .86 & $<.2$ & $<.2$ & $<.2$ & $<.2$ & $<.2$ & $<.2$ \\
\hline Richland Chambers Reservoir & Mid-lake & 315800096083001 & 07-08-99 & .33 & $<.2$ & .33 & $<.2$ & E.17 & $<.2$ & E. 17 \\
\hline Sam Rayburn Reservoir & PS & 310404094051101 & 07-14-99 & E.14 & $<.2$ & $<.2$ & $<.2$ & $<.2$ & $<.2$ & $<.2$ \\
\hline Somerville Lake & $\mathrm{AC}$ & 301908096313101 & 07-09-99 & 1.4 & $<.2$ & $<.2$ & $<.2$ & $<.2$ & $<.2$ & $<.2$ \\
\hline Toledo Bend Reservoir & PS & 311002093345501 & 07-14-99 & .27 & $<.2$ & .40 & $<.2$ & .50 & $<.2$ & .34 \\
\hline White River Reservoir & PS & 332747101052301 & 07-13-99 & .35 & $<.2$ & .51 & $<.2$ & .34 & $<.2$ & .23 \\
\hline
\end{tabular}


Table 2. Concentrations of soluble pesticides in public water-supply reservoirs sampled in Texas, June-July 1999

[ $\mu \mathrm{g} / \mathrm{L}$, micrograms per liter; <, less than; E, estimated; --, not analyzed]

\begin{tabular}{|c|c|c|c|c|c|c|c|c|c|}
\hline Site name & $\begin{array}{c}\text { Site } \\
\text { identifier }\end{array}$ & Station number & Date & $\begin{array}{c}\text { Acetochlor } \\
(\mu \mathrm{g} / \mathrm{L})\end{array}$ & $\begin{array}{c}\text { Acifluorfen } \\
(\mu g / L)\end{array}$ & $\begin{array}{c}\text { Alachlor } \\
(\mu \mathrm{g} / \mathrm{L})\end{array}$ & $\begin{array}{c}\text { Aldicarb } \\
(\mu \mathrm{g} / \mathrm{L})\end{array}$ & $\begin{array}{c}\text { Aldicarb } \\
\text { sulfone } \\
(\mu \mathrm{g} / \mathrm{L})\end{array}$ & $\begin{array}{c}\text { Aldicarb } \\
\text { sulfoxide } \\
(\mu \mathrm{g} / \mathrm{L})\end{array}$ \\
\hline Aquilla Lake & $\mathrm{AC}$ & 315358097122601 & 07-06-99 & $<0.002$ & $<0.035$ & 0.286 & $<0.55$ & $<0.1$ & $<0.021$ \\
\hline Buffalo Springs Lake & PS & 333205101414401 & 07-12-99 & $<.002$ & $<.035$ & $<.002$ & $<2.3$ & $<.1$ & $<.021$ \\
\hline Cedar Creek Reservoir & PS & 295502096440201 & 07-19-99 & $<.002$ & $<.035$ & $<.002$ & $<.55$ & $<.1$ & $<.54$ \\
\hline Choke Canyon Reservoir & $\mathrm{AC}$ & 8206890 & 07-07-99 & $<.002$ & $<.035$ & $<.002$ & $<.55$ & $<.1$ & $<.021$ \\
\hline Donna Reservoir & PS & 260912098040601 & 07-13-99 & $<.002$ & $<.035$ & $<.002$ & $<1.13$ & $<.14$ & $<.021$ \\
\hline E.V. Spence Reservoir & PS & 315535100341701 & $07-15-99$ & $<.002$ & $<.035$ & $<.002$ & $<.55$ & $<.67$ & $<.021$ \\
\hline Falcon International Reservoir & $\mathrm{BC}$ & 263815099111901 & 07-07-99 & $<.002$ & $<.035$ & $<.002$ & $<.55$ & $<.1$ & $<.021$ \\
\hline Fort Phantom Hill Reservoir & PS & 323632099410401 & 07-14-99 & $<.002$ & $<.035$ & $<.002$ & $<.55$ & $<.1$ & $<.021$ \\
\hline Granger Lake & $\mathrm{AC}$ & 304132097200801 & 06-30-99 & $<.002$ & $<.035$ & .158 & $<.55$ & $<.14$ & $<.021$ \\
\hline Grapevine Lake & $\mathrm{BC}$ & 325930097053801 & 07-26-99 & $<.002$ & $<.035$ & $<.002$ & $<.55$ & $<.1$ & $<.021$ \\
\hline Greenbelt Lake & $\mathrm{AC}$ & 350000100534701 & 07-13-99 & $<.002$ & $<.035$ & $<.002$ & $<.55$ & $<.1$ & $<.4$ \\
\hline Hubbard Creek Reservoir & P5 & 324913098581801 & $07-15-99$ & $<.002$ & $<.035$ & $<.002$ & $<.55$ & $<.1$ & $<.021$ \\
\hline La Feria Reservoir & PS & 260822097493401 & 07-14-99 & $<.002$ & $<.035$ & $<.002$ & $<2.93$ & $<.1$ & $<.021$ \\
\hline Lake Anahuac & PS & 294624094411201 & $07-21-99$ & $<.002$ & $<.035$ & $<.002$ & $<.55$ & $<.1$ & $<.021$ \\
\hline Lake Belton & $\mathrm{BC}$ & 310711097302201 & 06-30-99 & $<.002$ & $<.035$ & $<.002$ & $<.55$ & $<.1$ & $<.021$ \\
\hline Lake Bonham & PS & 333858096083301 & 07-27-99 & $<.002$ & $<.035$ & $<.002$ & $<.55$ & $<.1$ & $<.021$ \\
\hline Lake Bridgeport & Mid-lake & 331312097501801 & $07-21-99$ & $<.002$ & $<.035$ & $<.002$ & $<.55$ & $<.1$ & $<.021$ \\
\hline Lake Brownwood & PS & 315026099005301 & 07-19-99 & $<.002$ & $<.035$ & $<.002$ & $<.55$ & $<.27$ & $<.19$ \\
\hline Lake Houston & $\mathrm{BC}$ & 295702095091401 & 07-12-99 & $<.002$ & $<.035$ & $<.002$ & $<.55$ & $<.1$ & $<.021$ \\
\hline Lake J.B. Thomas & PS & 323510101085501 & 07-13-99 & $<.002$ & $<.035$ & $<.002$ & $<.55$ & $<.1$ & $<.021$ \\
\hline Lake Kickapoo & PS & 333913098480601 & 07-19-99 & $<.002$ & $<.035$ & $<.002$ & $<.55$ & $<.1$ & $<.021$ \\
\hline Lake Limestone & $\mathrm{BC}$ & 312458096205101 & 07-08-99 & $<.002$ & $<.035$ & $<.002$ & $<.55$ & $<.1$ & $<.021$ \\
\hline Lake Meredith & $\mathrm{BC}$ & 354113101360101 & 07-13-99 & $<.002$ & $<.035$ & $<.002$ & $<.55$ & $<.1$ & $<.021$ \\
\hline Lake Mexia & PS & 313848096345001 & 07-07-99 & $<.002$ & $<.035$ & $<.002$ & $<.55$ & $<.1$ & $<.021$ \\
\hline
\end{tabular}


Table 2. Concentrations of soluble pesticides in public water-supply reservoirs sampled in Texas, June-July 1999—Continued

\begin{tabular}{|c|c|c|c|c|c|c|c|c|c|}
\hline Site name & $\begin{array}{c}\text { Site } \\
\text { identifier }\end{array}$ & Station number & Date & $\begin{array}{l}\text { Acetochlor } \\
(\mu \mathrm{g} / \mathrm{L})\end{array}$ & $\begin{array}{c}\text { Acifluorfen } \\
(\mu \mathrm{g} / \mathrm{L})\end{array}$ & $\begin{array}{c}\text { Alachlor } \\
(\mu \mathrm{g} / \mathrm{L})\end{array}$ & $\begin{array}{c}\text { Aldicarb } \\
(\mu \mathrm{g} / \mathrm{L})\end{array}$ & $\begin{array}{c}\text { Aldicarb } \\
\text { sulfone } \\
(\mu \mathrm{g} / \mathrm{L})\end{array}$ & $\begin{array}{c}\text { Aldicarb } \\
\text { sulfoxide } \\
(\mu \mathrm{g} / \mathrm{L})\end{array}$ \\
\hline Lake Murvaul & PS & 320200094252501 & $07-15-99$ & $<0.002$ & $<0.035$ & $<0.002$ & $<0.55$ & $<0.1$ & $<0.021$ \\
\hline Lake Stamford & PS & 330414099340201 & 07-20-99 & $<.002$ & $<.035$ & $<.002$ & $<.55$ & $<.1$ & $<.021$ \\
\hline Lake Sweetwater & PS & 322609100181901 & 07-14-99 & $<.002$ & $<.035$ & $<.002$ & $<.55$ & $<.1$ & $<.021$ \\
\hline Lake Texana & $\mathrm{CC}$ & 285816096320201 & 07-20-99 & $<.002$ & $<.035$ & .030 & $<.57$ & $<.1$ & $<.021$ \\
\hline Lake Travis & PS & 302429097541401 & 06-28-99 & $<.002$ & $<.035$ & $<.002$ & $<.55$ & $<.1$ & $<.021$ \\
\hline Lake Tyler & PS & 321246095101701 & 07-16-99 & $<.002$ & $<.035$ & $<.002$ & $<.55$ & $<.57$ & $<.021$ \\
\hline Lake Waco & $\mathrm{AC}$ & 313430097113801 & 06-30-99 & $<.002$ & $<.035$ & $<.002$ & $<.55$ & $<.1$ & $<.021$ \\
\hline Lavon Lake & $\mathrm{AC}$ & 330203096284901 & 07-09-99 & $<.002$ & $<.035$ & .023 & $<.55$ & $<.1$ & $<.021$ \\
\hline Medina Lake & $\mathrm{AC}$ & 293225098560600 & 07-06-99 & $<.002$ & $<.035$ & $<.002$ & $<.55$ & $<.1$ & $<.021$ \\
\hline O.C. Fisher Lake & PS & 312909100293901 & 07-15-99 & $<.002$ & $<.035$ & $<.002$ & $<.55$ & $<.1$ & $<.021$ \\
\hline O.H. Ivie Reservoir & PS & 313130099391001 & $07-16-99$ & $<.002$ & $<.035$ & $<.002$ & $<.55$ & $<.3$ & $<.09$ \\
\hline Proctor Lake & $\mathrm{AC}$ & 315814098291201 & 07-20-99 & $<.002$ & $<.035$ & $<.002$ & $<.55$ & $<.1$ & $<.69$ \\
\hline Richland Chambers Reservoir & Mid-lake & 315800096083001 & 07-08-99 & $<.002$ & $<.035$ & $<.002$ & $<.55$ & $<.1$ & $<.021$ \\
\hline Sam Rayburn Reservoir & PS & 310404094051101 & 07-14-99 & $<.002$ & $<.035$ & $<.002$ & $<.55$ & $<.1$ & $<.021$ \\
\hline Somerville Lake & $\mathrm{AC}$ & 301908096313101 & 07-09-99 & $<.002$ & $<.035$ & $<.002$ & $<.55$ & $<.1$ & $<.021$ \\
\hline Toledo Bend Reservoir & PS & 311002093345501 & 07-14-99 & $<.002$ & $<.035$ & $<.002$ & $<.55$ & $<.1$ & $<.021$ \\
\hline White River Reservoir & PS & 332747101052301 & 07-13-99 & $<.002$ & $<.035$ & $<.002$ & $<.55$ & $<.1$ & $<.021$ \\
\hline
\end{tabular}


Table 2. Concentrations of soluble pesticides in public water-supply reservoirs sampled in Texas, June-July 1999-Continued

\begin{tabular}{|c|c|c|c|c|c|c|c|c|c|}
\hline Site name & $\begin{array}{c}\text { Site } \\
\text { identifier }\end{array}$ & Station number & Date & $\begin{array}{l}\text { alpha- } \\
\text { BHC } \\
(\mu \mathrm{g} / \mathrm{L})\end{array}$ & $\begin{array}{c}\text { Atrazine } \\
(\mu \mathrm{g} / \mathrm{L})\end{array}$ & $\begin{array}{c}\text { Azinphos- } \\
\text { methyl } \\
(\mu \mathrm{g} / \mathrm{L})\end{array}$ & $\begin{array}{c}\text { Benfluralin } \\
(\mu \mathrm{g} / L)\end{array}$ & $\begin{array}{c}\text { Bentazon } \\
(\mu \mathrm{g} / \mathrm{L})\end{array}$ & $\begin{array}{c}\text { Bromacil } \\
(\mu \mathrm{g} / \mathrm{L})\end{array}$ \\
\hline Aquilla Lake & $\mathrm{AC}$ & 315358097122601 & 07-06-99 & $<0.002$ & 1.32 & $<0.001$ & $<0.002$ & $<0.014$ & $<0.035$ \\
\hline Buffalo Springs Lake & PS & 333205101414401 & $07-12-99$ & $<.002$ & .263 & $<.001$ & $<.002$ & $<.014$ & $<.53$ \\
\hline Cedar Creek Reservoir & PS & 295502096440201 & 07-19-99 & $<.002$ & .053 & $<.001$ & $<.002$ & $<.014$ & $<.12$ \\
\hline Choke Canyon Reservoir & $\mathrm{AC}$ & 8206890 & 07-07-99 & $<.002$ & .048 & $<.001$ & $<.002$ & $<.014$ & $<.035$ \\
\hline Donna Reservoir & PS & 260912098040601 & $07-13-99$ & $<.002$ & .012 & E.033 & $<.002$ & $<.014$ & $<.035$ \\
\hline E.V. Spence Reservoir & PS & 315535100341701 & $07-15-99$ & $<.002$ & .031 & $<.001$ & $<.002$ & $<.014$ & $<.035$ \\
\hline Falcon International Reservoir & $\mathrm{BC}$ & 263815099111901 & 07-07-99 & $<.002$ & .009 & $<.001$ & $<.002$ & $<.014$ & $<.035$ \\
\hline Fort Phantom Hill Reservoir & PS & 323632099410401 & 07-14-99 & $<.002$ & .060 & $<.001$ & $<.002$ & $<.014$ & $<.46$ \\
\hline Granger Lake & $\mathrm{AC}$ & 304132097200801 & 06-30-99 & $<.002$ & 1.40 & $<.001$ & $<.002$ & $<.014$ & $<.035$ \\
\hline Grapevine Lake & $\mathrm{BC}$ & 325930097053801 & $07-26-99$ & $<.002$ & .284 & $<.001$ & $<.002$ & $<.014$ & $<1.3$ \\
\hline Greenbelt Lake & $\mathrm{AC}$ & 350000100534701 & $07-13-99$ & $<.002$ & .040 & $<.001$ & $<.002$ & $<.014$ & $<.035$ \\
\hline Hubbard Creek Reservoir & P5 & 324913098581801 & $07-15-99$ & $<.002$ & .012 & $<.001$ & $<.002$ & $<.014$ & $<.035$ \\
\hline La Feria Reservoir & PS & 260822097493401 & $07-14-99$ & $<.002$ & .010 & E.012 & $<.002$ & $<.014$ & $<.21$ \\
\hline Lake Anahuac & PS & 294624094411201 & $07-21-99$ & $<.002$ & .649 & $<.001$ & $<.002$ & $<.014$ & $<.05$ \\
\hline Lake Belton & $\mathrm{BC}$ & 310711097302201 & 06-30-99 & $<.002$ & .333 & $<.001$ & $<.002$ & $<.014$ & $<.12$ \\
\hline Lake Bonham & PS & 333858096083301 & $07-27-99$ & $<.002$ & .064 & $<.001$ & $<.002$ & $<.014$ & $<.15$ \\
\hline Lake Bridgeport & Mid-lake & 331312097501801 & $07-21-99$ & $<.002$ & .015 & $<.001$ & $<.002$ & $<.014$ & $<.035$ \\
\hline Lake Brownwood & PS & 315026099005301 & 07-19-99 & $<.002$ & .014 & $<.001$ & $<.002$ & $<.014$ & $<.035$ \\
\hline Lake Houston & $\mathrm{BC}$ & 295702095091401 & $07-12-99$ & $<.002$ & .270 & $<.001$ & $<.002$ & $<.014$ & $<.035$ \\
\hline Lake J.B. Thomas & PS & 323510101085501 & $07-13-99$ & $<.002$ & .013 & $<.001$ & $<.002$ & $<.014$ & $<.13$ \\
\hline Lake Kickapoo & PS & 333913098480601 & 07-19-99 & $<.002$ & .020 & $<.001$ & $<.002$ & $<.014$ & $<.035$ \\
\hline Lake Limestone & $\mathrm{BC}$ & 312458096205101 & 07-08-99 & $<.002$ & .068 & $<.001$ & $<.002$ & $<.014$ & $<.035$ \\
\hline Lake Meredith & $\mathrm{BC}$ & 354113101360101 & 07-13-99 & $<.002$ & .117 & $<.001$ & $<.002$ & $<.014$ & $<.1$ \\
\hline Lake Mexia & PS & 313848096345001 & 07-07-99 & $<.002$ & .674 & $<.001$ & $<.002$ & $<.014$ & $<.035$ \\
\hline
\end{tabular}


Table 2. Concentrations of soluble pesticides in public water-supply reservoirs sampled in Texas, June-July 1999-Continued

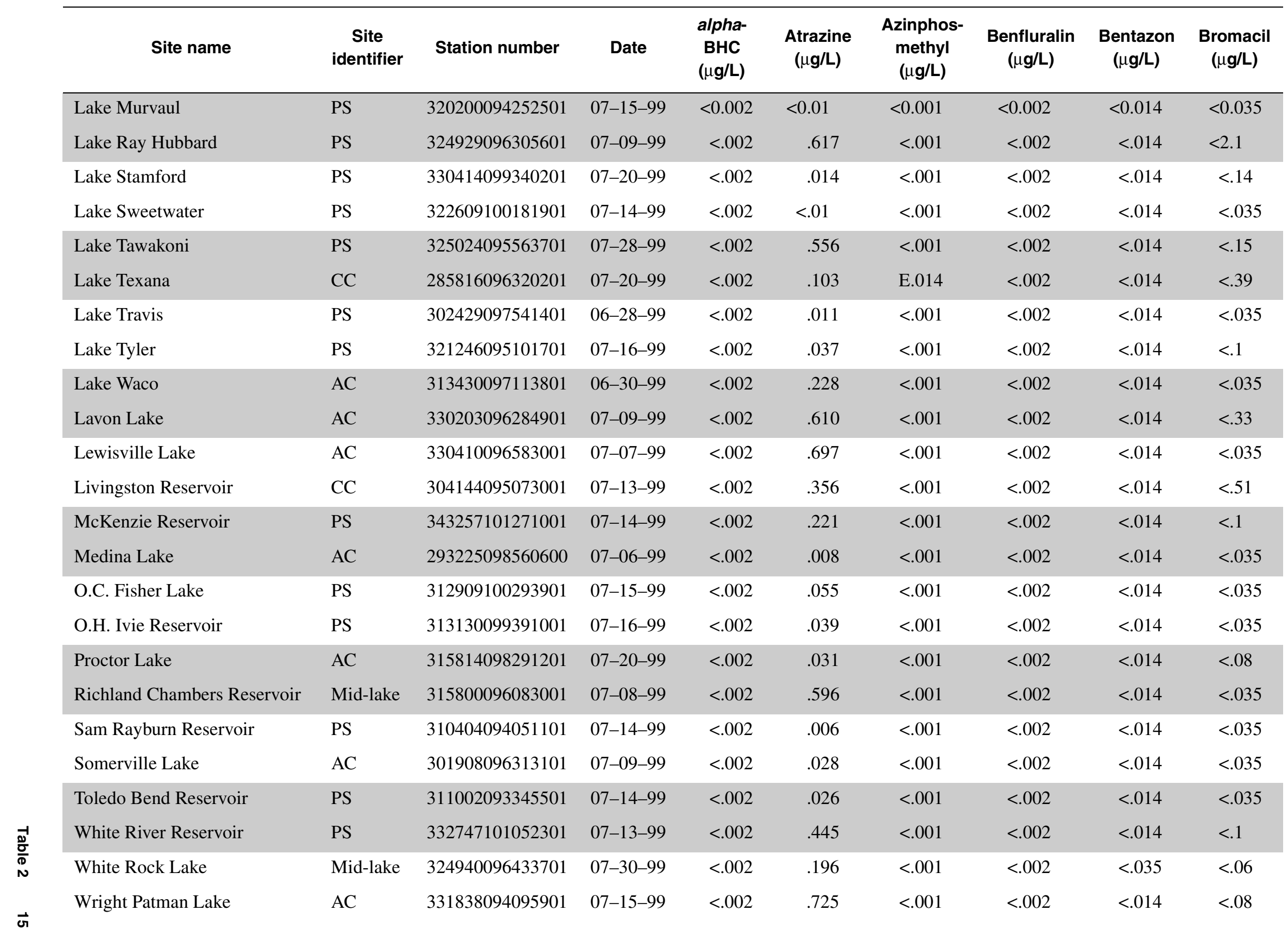


Table 2. Concentrations of soluble pesticides in public water-supply reservoirs sampled in Texas, June-July 1999-Continued

\begin{tabular}{|c|c|c|c|c|c|c|c|c|}
\hline Site name & $\begin{array}{c}\text { Site } \\
\text { identifier }\end{array}$ & Station number & Date & $\begin{array}{l}\text { Bromoxynil } \\
\quad(\mu \mathrm{g} / \mathrm{L})\end{array}$ & $\begin{array}{c}\text { Butylate } \\
(\mu \mathrm{g} / \mathrm{L})\end{array}$ & $\begin{array}{c}\text { Carbaryl } \\
(\mu \mathrm{g} / \mathrm{L})\end{array}$ & $\begin{array}{c}\text { Carbofuran } \\
(\mu g / L)\end{array}$ & $\begin{array}{c}\text { Chloramben } \\
(\mu \mathrm{g} / \mathrm{L})\end{array}$ \\
\hline Aquilla Lake & $\mathrm{AC}$ & 315358097122601 & 07-06-99 & $<0.035$ & $<0.002$ & $<0.008$ & $<0.12$ & $<0.42$ \\
\hline Buffalo Springs Lake & PS & 333205101414401 & 07-12-99 & $<.035$ & $<.002$ & $<.008$ & $<.12$ & $<.42$ \\
\hline Cedar Creek Reservoir & PS & 295502096440201 & 07-19-99 & $<.035$ & $<.002$ & $<.008$ & $<.12$ & $<.42$ \\
\hline Choke Canyon Reservoir & $\mathrm{AC}$ & 8206890 & 07-07-99 & $<.035$ & $<.002$ & $<.008$ & $<.12$ & $<.42$ \\
\hline Donna Reservoir & PS & 260912098040601 & 07-13-99 & $<.035$ & $<.002$ & $<.008$ & $<.12$ & $<.42$ \\
\hline E.V. Spence Reservoir & PS & 315535100341701 & 07-15-99 & $<.035$ & $<.002$ & $<.008$ & $<.12$ & $<.42$ \\
\hline Falcon International Reservoir & $\mathrm{BC}$ & 263815099111901 & 07-07-99 & $<.035$ & $<.002$ & $<.008$ & $<.12$ & $<.42$ \\
\hline Fort Phantom Hill Reservoir & PS & 323632099410401 & 07-14-99 & $<.035$ & $<.002$ & $<.008$ & $<.12$ & $<.42$ \\
\hline Granger Lake & $\mathrm{AC}$ & 304132097200801 & 06-30-99 & $<.035$ & $<.002$ & $<.008$ & $<.12$ & $<.42$ \\
\hline Grapevine Lake & $\mathrm{BC}$ & 325930097053801 & 07-26-99 & $<.035$ & $<.002$ & $<.008$ & $<.12$ & $<.42$ \\
\hline Greenbelt Lake & $\mathrm{AC}$ & 350000100534701 & 07-13-99 & $<.035$ & $<.002$ & $<.008$ & $<.12$ & $<.42$ \\
\hline Hubbard Creek Reservoir & P5 & 324913098581801 & $07-15-99$ & $<.035$ & $<.002$ & $<.008$ & $<.12$ & $<.42$ \\
\hline La Feria Reservoir & PS & 260822097493401 & 07-14-99 & $<.035$ & $<.002$ & $<.008$ & $<.12$ & $<.42$ \\
\hline Lake Anahuac & PS & 294624094411201 & $07-21-99$ & $<.035$ & $<.002$ & $<.008$ & $<.12$ & $<.42$ \\
\hline Lake Belton & $\mathrm{BC}$ & 310711097302201 & 06-30-99 & $<.035$ & $<.002$ & $<.008$ & $<.12$ & $<.42$ \\
\hline Lake Bonham & PS & 333858096083301 & 07-27-99 & $<.035$ & $<.002$ & $<.008$ & $<.12$ & -- \\
\hline Lake Bridgeport & Mid-lake & 331312097501801 & $07-21-99$ & $<.035$ & $<.002$ & $<.008$ & $<.12$ & $<.42$ \\
\hline Lake Brownwood & PS & 315026099005301 & 07-19-99 & $<.035$ & $<.002$ & $<.008$ & $<.12$ & $<.42$ \\
\hline Lake Houston & $\mathrm{BC}$ & 295702095091401 & $07-12-99$ & $<.035$ & $<.002$ & $<.008$ & $<.12$ & $<.42$ \\
\hline Lake J.B. Thomas & PS & 323510101085501 & 07-13-99 & $<.035$ & $<.002$ & $<.008$ & $<.12$ & $<.42$ \\
\hline Lake Kickapoo & PS & 333913098480601 & 07-19-99 & $<.035$ & $<.002$ & $<.008$ & $<.12$ & $<.42$ \\
\hline Lake Limestone & $\mathrm{BC}$ & 312458096205101 & 07-08-99 & $<.035$ & $<.002$ & $<.008$ & $<.12$ & $<.42$ \\
\hline Lake Meredith & $\mathrm{BC}$ & 354113101360101 & 07-13-99 & $<.035$ & $<.002$ & $<.008$ & $<.12$ & $<.42$ \\
\hline Lake Mexia & PS & 313848096345001 & 07-07-99 & $<.035$ & $<.002$ & $<.008$ & $<.12$ & $<.42$ \\
\hline
\end{tabular}


Table 2. Concentrations of soluble pesticides in public water-supply reservoirs sampled in Texas, June-July 1999-Continued

\begin{tabular}{|c|c|c|c|c|c|c|c|c|}
\hline Site name & $\begin{array}{c}\text { Site } \\
\text { identifier }\end{array}$ & Station number & Date & $\begin{array}{l}\text { Bromoxynil } \\
(\mu \mathrm{g} / \mathrm{L})\end{array}$ & $\begin{array}{c}\text { Butylate } \\
(\mu \mathrm{g} / \mathrm{L})\end{array}$ & $\begin{array}{c}\text { Carbaryl } \\
(\mu \mathrm{g} / \mathrm{L})\end{array}$ & $\begin{array}{l}\text { Carbofuran } \\
(\mu \mathbf{g} / L)\end{array}$ & $\begin{array}{c}\text { Chloramben } \\
(\mu \mathbf{g} / \mathrm{L})\end{array}$ \\
\hline Lake Murvaul & PS & 320200094252501 & $07-15-99$ & $<0.035$ & $<0.002$ & $<0.008$ & $<0.12$ & $<0.42$ \\
\hline Lake Ray Hubbard & PS & 324929096305601 & 07-09-99 & $<.035$ & $<.002$ & $<.008$ & $<.12$ & -- \\
\hline Lake Stamford & PS & 330414099340201 & 07-20-99 & $<.035$ & $<.002$ & $<.008$ & $<.12$ & $<.42$ \\
\hline Lake Sweetwater & PS & 322609100181901 & 07-14-99 & $<.035$ & $<.002$ & $<.008$ & $<.12$ & $<.42$ \\
\hline Lake Travis & PS & 302429097541401 & 06-28-99 & $<.035$ & $<.002$ & $<.008$ & $<.12$ & $<.42$ \\
\hline Lake Tyler & PS & 321246095101701 & 07-16-99 & $<.035$ & $<.002$ & $<.008$ & $<.12$ & $<.42$ \\
\hline Lake Waco & $\mathrm{AC}$ & 313430097113801 & 06-30-99 & $<.035$ & $<.002$ & $<.008$ & $<.12$ & $<.42$ \\
\hline Lavon Lake & $\mathrm{AC}$ & 330203096284901 & 07-09-99 & $<.035$ & $<.002$ & $<.008$ & $<.12$ & $<.42$ \\
\hline Medina Lake & $\mathrm{AC}$ & 293225098560600 & 07-06-99 & $<.035$ & $<.002$ & $<.008$ & $<.12$ & $<.42$ \\
\hline O.C. Fisher Lake & PS & 312909100293901 & $07-15-99$ & $<.035$ & $<.002$ & $<.008$ & $<.12$ & $<.42$ \\
\hline O.H. Ivie Reservoir & PS & 313130099391001 & $07-16-99$ & $<.035$ & $<.002$ & $<.008$ & $<.12$ & $<.42$ \\
\hline Proctor Lake & $\mathrm{AC}$ & 315814098291201 & 07-20-99 & $<.035$ & $<.002$ & $<.008$ & $<.12$ & $<.42$ \\
\hline Richland Chambers Reservoir & Mid-lake & 315800096083001 & 07-08-99 & $<.035$ & $<.002$ & $<.008$ & $<.12$ & $<.42$ \\
\hline Sam Rayburn Reservoir & PS & 310404094051101 & 07-14-99 & $<.035$ & $<.002$ & $<.008$ & $<.12$ & $<.42$ \\
\hline Somerville Lake & $\mathrm{AC}$ & 301908096313101 & 07-09-99 & $<.035$ & $<.002$ & $<.008$ & $<.12$ & $<.42$ \\
\hline Toledo Bend Reservoir & PS & 311002093345501 & 07-14-99 & $<.035$ & $<.002$ & $<.008$ & $<.12$ & $<.42$ \\
\hline White River Reservoir & PS & 332747101052301 & 07-13-99 & $<.035$ & $<.002$ & $<.008$ & $<.12$ & $<.42$ \\
\hline
\end{tabular}


Table 2. Concentrations of soluble pesticides in public water-supply reservoirs sampled in Texas, June-July 1999-Continued

\begin{tabular}{|c|c|c|c|c|c|c|c|}
\hline Site name & $\begin{array}{c}\text { Site } \\
\text { identifier }\end{array}$ & Station number & Date & $\begin{array}{c}\text { Chlorothalonil } \\
(\mu \mathrm{g} / \mathrm{L})\end{array}$ & $\begin{array}{l}\text { Chlorpyrifos } \\
(\mu \mathrm{g} / \mathrm{L})\end{array}$ & $\begin{array}{c}\text { Clopyralid } \\
(\mu g / L)\end{array}$ & $\begin{array}{c}\text { Cyanazine } \\
(\mu \mathrm{g} / \mathrm{L})\end{array}$ \\
\hline Aquilla Lake & $\mathrm{AC}$ & 315358097122601 & $07-06-99$ & $<0.48$ & $<0.004$ & $<0.23$ & $<0.004$ \\
\hline Buffalo Springs Lake & PS & 333205101414401 & 07-12-99 & $<.48$ & $<.004$ & $<.23$ & $<.004$ \\
\hline Cedar Creek Reservoir & PS & 295502096440201 & 07-19-99 & $<.48$ & $<.004$ & $<.23$ & $<.004$ \\
\hline Choke Canyon Reservoir & $\mathrm{AC}$ & 8206890 & 07-07-99 & $<.48$ & E.003 & $<.23$ & $<.004$ \\
\hline Donna Reservoir & PS & 260912098040601 & 07-13-99 & $<.48$ & $<.004$ & $<.23$ & $<.004$ \\
\hline E.V. Spence Reservoir & PS & 315535100341701 & $07-15-99$ & $<.48$ & $<.004$ & $<.23$ & $<.004$ \\
\hline Falcon International Reservoir & $\mathrm{BC}$ & 263815099111901 & 07-07-99 & $<.48$ & $<.004$ & $<.23$ & $<.004$ \\
\hline Fort Phantom Hill Reservoir & PS & 323632099410401 & 07-14-99 & $<.48$ & $<.004$ & $<.23$ & $<.004$ \\
\hline Granger Lake & $\mathrm{AC}$ & 304132097200801 & 06-30-99 & $<.48$ & $<.004$ & $<.23$ & $<.004$ \\
\hline Grapevine Lake & $\mathrm{BC}$ & 325930097053801 & 07-26-99 & $<.48$ & $<.004$ & $<.23$ & $<.004$ \\
\hline Greenbelt Lake & $\mathrm{AC}$ & 350000100534701 & 07-13-99 & $<.48$ & $<.004$ & $<.23$ & $<.004$ \\
\hline Hubbard Creek Reservoir & P5 & 324913098581801 & $07-15-99$ & $<.48$ & $<.004$ & $<.23$ & $<.004$ \\
\hline La Feria Reservoir & PS & 260822097493401 & 07-14-99 & $<.48$ & $<.004$ & $<.23$ & $<.004$ \\
\hline Lake Anahuac & PS & 294624094411201 & $07-21-99$ & $<.48$ & $<.004$ & $<.23$ & $<.004$ \\
\hline Lake Belton & $\mathrm{BC}$ & 310711097302201 & 06-30-99 & $<.48$ & $<.004$ & $<.23$ & $<.004$ \\
\hline Lake Bonham & PS & 333858096083301 & $07-27-99$ & $<.48$ & $<.004$ & $<.23$ & $<.004$ \\
\hline Lake Bridgeport & Mid-lake & 331312097501801 & $07-21-99$ & $<.48$ & $<.004$ & $<.23$ & $<.004$ \\
\hline Lake Brownwood & PS & 315026099005301 & 07-19-99 & $<.48$ & $<.004$ & $<.23$ & $<.004$ \\
\hline Lake Houston & $\mathrm{BC}$ & 295702095091401 & 07-12-99 & $<.48$ & $<.004$ & $<.23$ & $<.004$ \\
\hline Lake J.B. Thomas & PS & 323510101085501 & 07-13-99 & $<.48$ & $<.004$ & $<.23$ & $<.004$ \\
\hline Lake Kickapoo & PS & 333913098480601 & 07-19-99 & $<.48$ & $<.004$ & $<.23$ & $<.004$ \\
\hline Lake Limestone & $\mathrm{BC}$ & 312458096205101 & 07-08-99 & $<.48$ & $<.004$ & $<.23$ & $<.004$ \\
\hline Lake Meredith & $\mathrm{BC}$ & 354113101360101 & 07-13-99 & $<.48$ & $<.004$ & $<.23$ & $<.004$ \\
\hline Lake Mexia & PS & 313848096345001 & 07-07-99 & $<.48$ & $<.004$ & $<.23$ & $<.004$ \\
\hline
\end{tabular}


Table 2. Concentrations of soluble pesticides in public water-supply reservoirs sampled in Texas, June-July 1999-Continued

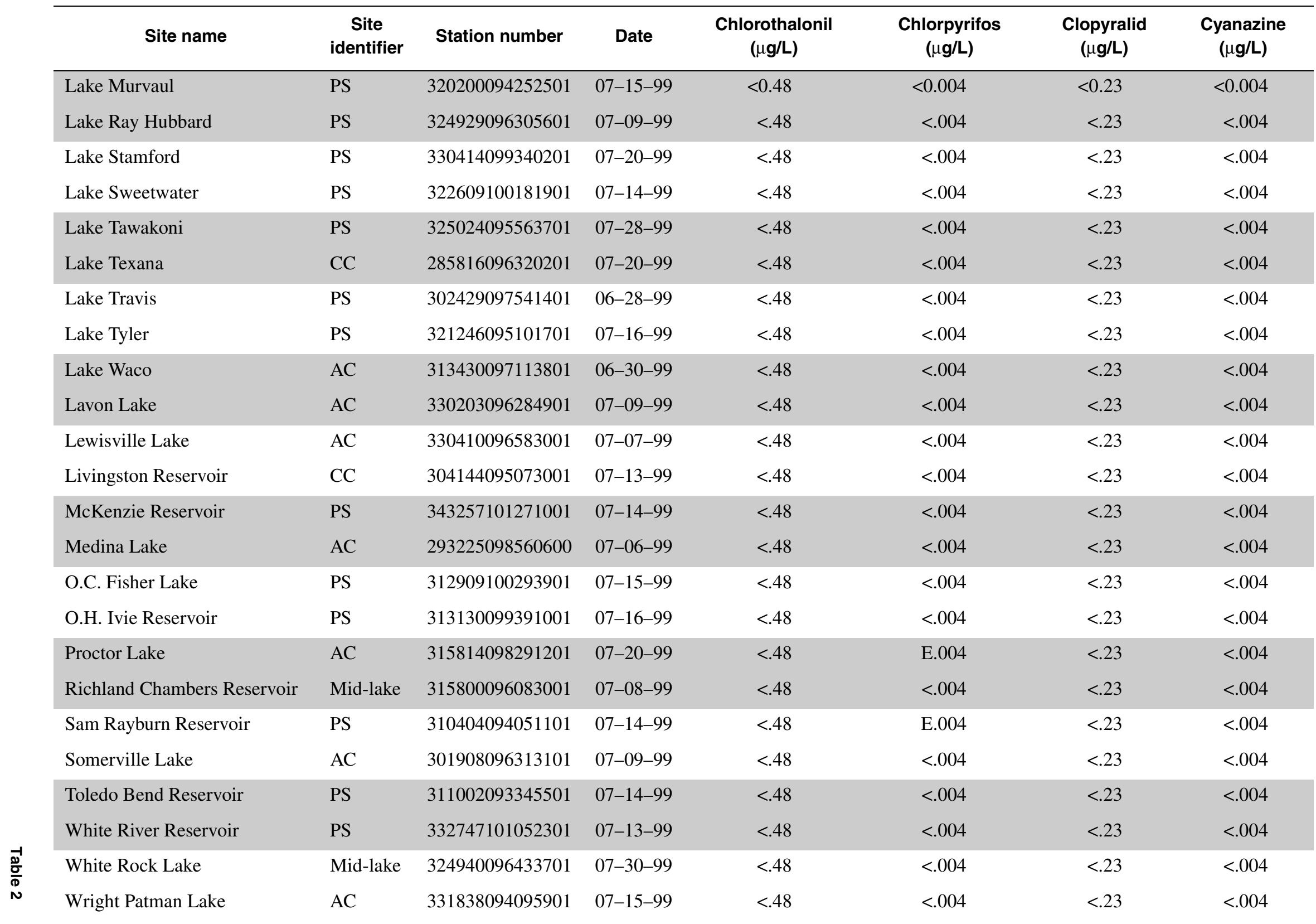


Table 2. Concentrations of soluble pesticides in public water-supply reservoirs sampled in Texas, June-July 1999-Continued

\begin{tabular}{|c|c|c|c|c|c|c|c|c|c|}
\hline Site name & $\begin{array}{c}\text { Site } \\
\text { identifier }\end{array}$ & Station number & Date & $\begin{array}{c}\text { Dacthal } \\
\text { mono-acid } \\
(\mu \mathrm{g} / \mathrm{L})\end{array}$ & $\begin{array}{l}2,4-D \\
(\mu \mathrm{g} / L)\end{array}$ & $\begin{array}{c}2,4-D B \\
(\mu \mathrm{g} / \mathrm{L})\end{array}$ & $\begin{array}{l}\text { DCPA } \\
(\mu \mathrm{g} / \mathrm{L})\end{array}$ & $\begin{array}{c}\text { Deethyl- } \\
\text { atrazine } \\
(\mu \mathrm{g} / \mathrm{L})\end{array}$ & $\begin{array}{c}\text { Diazinon } \\
(\mu \mathrm{g} / \mathrm{L})\end{array}$ \\
\hline Aquilla Lake & $\mathrm{AC}$ & 315358097122601 & 07-06-99 & $<0.017$ & $<0.15$ & $<0.24$ & $<0.002$ & E0.311 & $<0.002$ \\
\hline Buffalo Springs Lake & PS & 333205101414401 & $07-12-99$ & $<.017$ & .25 & $<.24$ & $<.002$ & E.039 & .043 \\
\hline Cedar Creek Reservoir & PS & 295502096440201 & 07-19-99 & $<.017$ & $<.15$ & $<.24$ & $<.002$ & E.025 & $<.002$ \\
\hline Choke Canyon Reservoir & $\mathrm{AC}$ & 8206890 & 07-07-99 & $<.017$ & $<.15$ & $<.24$ & $<.002$ & E.015 & $<.002$ \\
\hline Donna Reservoir & PS & 260912098040601 & 07-13-99 & $<.017$ & $<.15$ & $<.24$ & $<.002$ & E.003 & $<.002$ \\
\hline E.V. Spence Reservoir & PS & 315535100341701 & $07-15-99$ & $<.017$ & $<.15$ & $<.24$ & $<.002$ & E.010 & $<.002$ \\
\hline Falcon International Reservoir & $\mathrm{BC}$ & 263815099111901 & 07-07-99 & $<.017$ & $<.15$ & $<.24$ & $<.002$ & $<.002$ & $<.002$ \\
\hline Fort Phantom Hill Reservoir & PS & 323632099410401 & 07-14-99 & $<.017$ & $<.15$ & $<.24$ & $<.002$ & E.016 & $<.002$ \\
\hline Granger Lake & $\mathrm{AC}$ & 304132097200801 & 06-30-99 & $<.017$ & .12 & $<.24$ & $<.002$ & E.194 & .007 \\
\hline Grapevine Lake & $\mathrm{BC}$ & 325930097053801 & 07-26-99 & $<.017$ & $<.15$ & $<.24$ & $<.002$ & E.055 & .010 \\
\hline Greenbelt Lake & $\mathrm{AC}$ & 350000100534701 & 07-13-99 & $<.017$ & $<.15$ & $<.24$ & $<.002$ & E.016 & $<.002$ \\
\hline Hubbard Creek Reservoir & P5 & 324913098581801 & $07-15-99$ & $<.017$ & $<.15$ & $<.24$ & $<.002$ & E.006 & $<.002$ \\
\hline La Feria Reservoir & PS & 260822097493401 & 07-14-99 & $<.017$ & $<.15$ & $<.24$ & $<.002$ & E.004 & $<.002$ \\
\hline Lake Anahuac & PS & 294624094411201 & $07-21-99$ & $<.017$ & $<.15$ & $<.24$ & $<.002$ & E.089 & $<.002$ \\
\hline Lake Belton & $\mathrm{BC}$ & 310711097302201 & 06-30-99 & $<.017$ & $<.15$ & $<.24$ & $<.002$ & E.036 & $<.002$ \\
\hline Lake Bonham & PS & 333858096083301 & 07-27-99 & $<.017$ & $<.15$ & $<.24$ & $<.002$ & E.019 & $<.002$ \\
\hline Lake Bridgeport & Mid-lake & 331312097501801 & 07-21-99 & $<.017$ & $<.15$ & $<.24$ & $<.002$ & E.008 & $<.002$ \\
\hline Lake Brownwood & PS & 315026099005301 & 07-19-99 & $<.017$ & $<.15$ & $<.24$ & $<.002$ & E.007 & $<.002$ \\
\hline Lake Houston & $\mathrm{BC}$ & 295702095091401 & 07-12-99 & $<.017$ & $<.15$ & $<.24$ & $<.002$ & E.027 & .036 \\
\hline Lake J.B. Thomas & PS & 323510101085501 & 07-13-99 & $<.017$ & $<.15$ & $<.24$ & $<.002$ & $<.002$ & $<.002$ \\
\hline Lake Kickapoo & PS & 333913098480601 & 07-19-99 & $<.017$ & $<.15$ & $<.24$ & $<.002$ & E.009 & $<.002$ \\
\hline Lake Limestone & $\mathrm{BC}$ & 312458096205101 & 07-08-99 & $<.017$ & $<.15$ & $<.24$ & $<.002$ & E.011 & .004 \\
\hline Lake Meredith & $\mathrm{BC}$ & 354113101360101 & 07-13-99 & $<.017$ & $<.15$ & $<.24$ & $<.002$ & E.034 & $<.002$ \\
\hline Lake Mexia & PS & 313848096345001 & 07-07-99 & $<.017$ & $<.15$ & $<.24$ & $<.002$ & E.095 & $<.002$ \\
\hline
\end{tabular}


Table 2. Concentrations of soluble pesticides in public water-supply reservoirs sampled in Texas, June-July 1999—Continued

\begin{tabular}{|c|c|c|c|c|c|c|c|c|c|}
\hline Site name & $\begin{array}{c}\text { Site } \\
\text { identifier }\end{array}$ & Station number & Date & $\begin{array}{c}\text { Dacthal } \\
\text { mono-acid } \\
(\mu \mathrm{g} / \mathrm{L})\end{array}$ & $\begin{array}{l}2,4-D \\
(\mu g / L)\end{array}$ & $\begin{array}{c}2,4-D B \\
(\mu \mathrm{g} / L)\end{array}$ & $\begin{array}{l}\text { DCPA } \\
(\mu \mathrm{g} / \mathrm{L})\end{array}$ & $\begin{array}{c}\text { Deethyl- } \\
\text { atrazine } \\
(\mu \mathrm{g} / \mathrm{L})\end{array}$ & $\begin{array}{c}\text { Diazinon } \\
(\mu \mathrm{g} / \mathrm{L})\end{array}$ \\
\hline Lake Murvaul & PS & 320200094252501 & $07-15-99$ & $<0.017$ & $<0.15$ & $<0.24$ & $<0.002$ & $<0.002$ & $<0.002$ \\
\hline Lake Ray Hubbard & PS & 324929096305601 & 07-09-99 & $<.017$ & $<.15$ & $<.24$ & $<.002$ & E.195 & .043 \\
\hline Lake Stamford & PS & 330414099340201 & 07-20-99 & $<.017$ & $<.15$ & $<.24$ & $<.002$ & E.009 & $<.002$ \\
\hline Lake Sweetwater & PS & 322609100181901 & 07-14-99 & $<.017$ & $<.15$ & $<.24$ & $<.002$ & $<.002$ & $<.002$ \\
\hline Lake Tawakoni & PS & 325024095563701 & 07-28-99 & $<.017$ & $<.15$ & $<.24$ & $<.002$ & E.086 & $<.002$ \\
\hline Lake Texana & $\mathrm{CC}$ & 285816096320201 & 07-20-99 & $<.017$ & $<.15$ & $<.24$ & $<.002$ & E.023 & $<.002$ \\
\hline Lake Travis & PS & 302429097541401 & 06-28-99 & $<.017$ & $<.15$ & $<.24$ & E.001 & E.003 & $<.002$ \\
\hline Lake Tyler & PS & 321246095101701 & 07-16-99 & $<.017$ & $<.15$ & $<.24$ & $<.002$ & E.006 & .014 \\
\hline Lake Waco & $\mathrm{AC}$ & 313430097113801 & 06-30-99 & $<.017$ & $<.15$ & $<.24$ & $<.002$ & E.060 & $<.002$ \\
\hline Lavon Lake & $\mathrm{AC}$ & 330203096284901 & 07-09-99 & $<.017$ & $<.15$ & $<.24$ & $<.002$ & E.159 & .006 \\
\hline Lewisville Lake & $\mathrm{AC}$ & 330410096583001 & 07-07-99 & $<.017$ & $<.15$ & $<.24$ & $<.002$ & E. 120 & .012 \\
\hline Livingston Reservoir & $\mathrm{CC}$ & 304144095073001 & 07-13-99 & $<.017$ & $<.15$ & $<.24$ & $<.002$ & E.062 & .011 \\
\hline McKenzie Reservoir & PS & 343257101271001 & 07-14-99 & $<.017$ & $<.15$ & $<.24$ & $<.002$ & E. 170 & $<.002$ \\
\hline Medina Lake & $\mathrm{AC}$ & 293225098560600 & 07-06-99 & $<.017$ & $<.15$ & $<.24$ & $<.002$ & E.001 & $<.002$ \\
\hline O.C. Fisher Lake & PS & 312909100293901 & 07-15-99 & $<.017$ & $<.15$ & $<.24$ & $<.002$ & E.021 & $<.002$ \\
\hline O.H. Ivie Reservoir & PS & 313130099391001 & 07-16-99 & $<.017$ & $<.15$ & $<.24$ & $<.002$ & E.027 & $<.002$ \\
\hline Proctor Lake & $\mathrm{AC}$ & 315814098291201 & 07-20-99 & $<.017$ & $<.15$ & $<.24$ & $<.002$ & E.009 & $<.002$ \\
\hline Richland Chambers Reservoir & Mid-lake & 315800096083001 & 07-08-99 & $<.017$ & $<.15$ & $<.24$ & $<.002$ & E.149 & .018 \\
\hline Sam Rayburn Reservoir & PS & 310404094051101 & 07-14-99 & $<.017$ & $<.15$ & $<.24$ & $<.002$ & $<.002$ & $<.002$ \\
\hline Somerville Lake & $\mathrm{AC}$ & 301908096313101 & 07-09-99 & $<.017$ & $<.15$ & $<.24$ & $<.002$ & E.007 & $<.002$ \\
\hline Toledo Bend Reservoir & PS & 311002093345501 & 07-14-99 & $<.017$ & $<.15$ & $<.24$ & $<.002$ & E.009 & $<.002$ \\
\hline White River Reservoir & PS & 332747101052301 & 07-13-99 & $<.017$ & $<.15$ & $<.24$ & $<.002$ & E.064 & $<.002$ \\
\hline White Rock Lake & Mid-lake & 324940096433701 & 07-30-99 & $<.039$ & $<.11$ & $<.1$ & $<.002$ & E.047 & .065 \\
\hline Wright Patman Lake & $\mathrm{AC}$ & 331838094095901 & $07-15-99$ & $<.017$ & $<.15$ & $<.24$ & $<.002$ & E. 100 & $<.002$ \\
\hline
\end{tabular}


Table 2. Concentrations of soluble pesticides in public water-supply reservoirs sampled in Texas, June-July 1999-Continued

\begin{tabular}{|c|c|c|c|c|c|c|c|c|}
\hline Site name & $\begin{array}{c}\text { Site } \\
\text { identifier }\end{array}$ & Station number & Date & $\begin{array}{c}\text { Dicamba } \\
(\mu \mathrm{g} / \mathrm{L})\end{array}$ & $\begin{array}{l}\text { Dichlobenil } \\
(\mu \mathrm{g} / \mathrm{L})\end{array}$ & $\begin{array}{c}\text { Dichloroprop } \\
(\mu \mathrm{g} / \mathrm{L})\end{array}$ & $\begin{array}{c}\text { Dieldrin } \\
(\mu \mathrm{g} / \mathrm{L})\end{array}$ & $\begin{array}{c}\text { 2, 6-Diethyl- } \\
\text { aniline } \\
(\mu \mathrm{g} / \mathrm{L})\end{array}$ \\
\hline Aquilla Lake & $\mathrm{AC}$ & 315358097122601 & $07-06-99$ & $<0.035$ & $<1.2$ & $<0.032$ & $<0.001$ & $<0.003$ \\
\hline Buffalo Springs Lake & PS & 333205101414401 & $07-12-99$ & $<.035$ & $<1.2$ & $<.032$ & $<.001$ & $<.003$ \\
\hline Cedar Creek Reservoir & PS & 295502096440201 & 07-19-99 & $<.035$ & $<1.2$ & $<.032$ & $<.001$ & $<.003$ \\
\hline Choke Canyon Reservoir & $\mathrm{AC}$ & 8206890 & 07-07-99 & $<.035$ & $<1.2$ & $<.032$ & $<.001$ & $<.003$ \\
\hline Donna Reservoir & PS & 260912098040601 & 07-13-99 & $<.035$ & $<1.2$ & $<.032$ & $<.001$ & $<.003$ \\
\hline E.V. Spence Reservoir & PS & 315535100341701 & $07-15-99$ & $<.035$ & $<1.2$ & $<.032$ & $<.001$ & $<.003$ \\
\hline Falcon International Reservoir & $\mathrm{BC}$ & 263815099111901 & 07-07-99 & $<.035$ & $<1.2$ & $<.032$ & $<.001$ & $<.003$ \\
\hline Fort Phantom Hill Reservoir & PS & 323632099410401 & 07-14-99 & $<.035$ & $<1.2$ & $<.032$ & $<.001$ & $<.003$ \\
\hline Granger Lake & $\mathrm{AC}$ & 304132097200801 & 06-30-99 & $<.035$ & $<1.2$ & $<.032$ & $<.001$ & $<.003$ \\
\hline Grapevine Lake & $\mathrm{BC}$ & 325930097053801 & $07-26-99$ & $<.035$ & $<1.2$ & $<.032$ & $<.001$ & $<.003$ \\
\hline Greenbelt Lake & $\mathrm{AC}$ & 350000100534701 & 07-13-99 & $<.035$ & $<1.2$ & $<.032$ & $<.001$ & $<.003$ \\
\hline Hubbard Creek Reservoir & P5 & 324913098581801 & $07-15-99$ & $<.035$ & $<1.2$ & $<.032$ & $<.001$ & $<.003$ \\
\hline La Feria Reservoir & PS & 260822097493401 & 07-14-99 & $<.035$ & $<1.2$ & $<.032$ & .007 & $<.003$ \\
\hline Lake Anahuac & PS & 294624094411201 & $07-21-99$ & $<.035$ & $<1.2$ & $<.032$ & $<.001$ & $<.003$ \\
\hline Lake Belton & $\mathrm{BC}$ & 310711097302201 & 06-30-99 & $<.035$ & $<1.2$ & $<.032$ & $<.001$ & $<.003$ \\
\hline Lake Bonham & PS & 333858096083301 & 07-27-99 & $<.035$ & $<1.2$ & $<.032$ & $<.001$ & $<.003$ \\
\hline Lake Bridgeport & Mid-lake & 331312097501801 & $07-21-99$ & $<.035$ & $<1.2$ & $<.032$ & $<.001$ & $<.003$ \\
\hline Lake Brownwood & PS & 315026099005301 & 07-19-99 & $<.035$ & $<1.2$ & $<.032$ & $<.001$ & $<.003$ \\
\hline Lake Houston & $\mathrm{BC}$ & 295702095091401 & 07-12-99 & $<.035$ & $<1.2$ & $<.032$ & $<.001$ & $<.003$ \\
\hline Lake J.B. Thomas & PS & 323510101085501 & 07-13-99 & $<.11$ & $<1.2$ & $<.032$ & $<.001$ & $<.003$ \\
\hline Lake Kickapoo & PS & 333913098480601 & 07-19-99 & $<.035$ & $<1.2$ & $<.032$ & $<.001$ & $<.003$ \\
\hline Lake Limestone & $\mathrm{BC}$ & 312458096205101 & 07-08-99 & $<.035$ & $<1.2$ & $<.032$ & $<.001$ & $<.003$ \\
\hline Lake Meredith & $\mathrm{BC}$ & 354113101360101 & 07-13-99 & $<.035$ & $<1.2$ & $<.032$ & $<.001$ & $<.003$ \\
\hline Lake Mexia & PS & 313848096345001 & 07-07-99 & $<.035$ & $<1.2$ & $<.032$ & $<.001$ & $<.003$ \\
\hline
\end{tabular}


Table 2. Concentrations of soluble pesticides in public water-supply reservoirs sampled in Texas, June-July 1999—Continued

\begin{tabular}{|c|c|c|c|c|c|c|c|c|}
\hline Site name & $\begin{array}{c}\text { Site } \\
\text { identifier }\end{array}$ & Station number & Date & $\begin{array}{c}\text { Dicamba } \\
(\mu \mathbf{g} / L)\end{array}$ & $\begin{array}{l}\text { Dichlobenil } \\
(\mu \mathrm{g} / L)\end{array}$ & $\begin{array}{c}\text { Dichloroprop } \\
(\mu \mathrm{g} / \mathrm{L})\end{array}$ & $\begin{array}{c}\text { Dieldrin } \\
(\mu \mathrm{g} / \mathrm{L})\end{array}$ & $\begin{array}{c}\text { 2, 6-Diethyl- } \\
\text { aniline } \\
(\mu \mathrm{g} / \mathrm{L})\end{array}$ \\
\hline Lake Murvaul & PS & 320200094252501 & $07-15-99$ & $<0.035$ & $<1.2$ & $<0.032$ & $<0.001$ & $<0.003$ \\
\hline Lake Ray Hubbard & PS & 324929096305601 & 07-09-99 & $<.035$ & $<1.2$ & $<.032$ & $<.001$ & $<.003$ \\
\hline Lake Stamford & PS & 330414099340201 & 07-20-99 & $<.035$ & $<1.2$ & $<.032$ & $<.001$ & $<.003$ \\
\hline Lake Sweetwater & PS & 322609100181901 & 07-14-99 & $<.035$ & $<1.2$ & $<.032$ & $<.001$ & $<.003$ \\
\hline Lake Tawakoni & PS & 325024095563701 & 07-28-99 & $<.035$ & $<1.2$ & $<.032$ & $<.001$ & $<.003$ \\
\hline Lake Texana & $\mathrm{CC}$ & 285816096320201 & 07-20-99 & $<.035$ & $<1.2$ & $<.11$ & $<.001$ & $<.003$ \\
\hline Lake Travis & PS & 302429097541401 & 06-28-99 & $<.035$ & $<1.2$ & $<.032$ & $<.001$ & $<.003$ \\
\hline Lake Tyler & PS & 321246095101701 & 07-16-99 & $<.11$ & $<1.2$ & $<.032$ & $<.001$ & $<.003$ \\
\hline Lake Waco & $\mathrm{AC}$ & 313430097113801 & 06-30-99 & $<.035$ & $<1.2$ & $<.032$ & $<.001$ & $<.003$ \\
\hline Lavon Lake & $\mathrm{AC}$ & 330203096284901 & 07-09-99 & $<.035$ & $<1.2$ & $<.032$ & $<.001$ & $<.003$ \\
\hline Lewisville Lake & $\mathrm{AC}$ & 330410096583001 & 07-07-99 & $<.035$ & $<1.2$ & $<.032$ & $<.001$ & $<.003$ \\
\hline Livingston Reservoir & $\mathrm{CC}$ & 304144095073001 & 07-13-99 & $<.035$ & $<1.2$ & $<.032$ & $<.001$ & $<.003$ \\
\hline McKenzie Reservoir & PS & 343257101271001 & 07-14-99 & $<.035$ & $<1.2$ & $<.032$ & $<.001$ & $<.003$ \\
\hline Medina Lake & $\mathrm{AC}$ & 293225098560600 & 07-06-99 & $<.035$ & $<1.2$ & $<.032$ & $<.001$ & $<.003$ \\
\hline O.C. Fisher Lake & PS & 312909100293901 & $07-15-99$ & $<.035$ & $<1.2$ & $<.032$ & $<.001$ & $<.003$ \\
\hline O.H. Ivie Reservoir & PS & 313130099391001 & 07-16-99 & $<.035$ & $<1.2$ & $<.032$ & $<.001$ & $<.003$ \\
\hline Proctor Lake & $\mathrm{AC}$ & 315814098291201 & $07-20-99$ & $<.035$ & $<1.2$ & $<.032$ & $<.001$ & $<.003$ \\
\hline Richland Chambers Reservoir & Mid-lake & 315800096083001 & 07-08-99 & $<.035$ & $<1.2$ & $<.032$ & $<.001$ & $<.003$ \\
\hline Sam Rayburn Reservoir & PS & 310404094051101 & 07-14-99 & $<.14$ & $<1.2$ & $<.032$ & $<.001$ & $<.003$ \\
\hline Somerville Lake & $\mathrm{AC}$ & 301908096313101 & 07-09-99 & $<.035$ & $<1.2$ & $<.032$ & $<.001$ & $<.003$ \\
\hline Toledo Bend Reservoir & PS & 311002093345501 & 07-14-99 & $<.035$ & $<1.2$ & $<.032$ & $<.001$ & $<.003$ \\
\hline White River Reservoir & PS & 332747101052301 & 07-13-99 & $<.035$ & $<1.2$ & $<.032$ & $<.001$ & $<.003$ \\
\hline White Rock Lake & Mid-lake & 324940096433701 & 07-30-99 & $<.043$ & $<.07$ & $<.032$ & $<.001$ & $<.003$ \\
\hline Wright Patman Lake & $\mathrm{AC}$ & 331838094095901 & $07-15-99$ & $<.15$ & $<1.2$ & $<.032$ & $<.001$ & $<.003$ \\
\hline
\end{tabular}


Table 2. Concentrations of soluble pesticides in public water-supply reservoirs sampled in Texas, June-July 1999—Continued

\begin{tabular}{|c|c|c|c|c|c|c|c|c|}
\hline Site name & $\begin{array}{c}\text { Site } \\
\text { identifier }\end{array}$ & Station number & Date & $\begin{array}{c}\text { Dinoseb } \\
(\mu \mathrm{g} / \mathrm{L})\end{array}$ & $\begin{array}{l}\text { Disulfoton } \\
(\mu \mathrm{g} / \mathrm{L})\end{array}$ & $\begin{array}{c}\text { Diuron } \\
(\mu \mathrm{g} / \mathrm{L})\end{array}$ & $\begin{array}{l}\text { DNOC } \\
(\mu \mathrm{g} / \mathrm{L})\end{array}$ & $\begin{array}{l}\text { EPTC } \\
(\mu \mathrm{g} / \mathrm{L})\end{array}$ \\
\hline Aquilla Lake & $\mathrm{AC}$ & 315358097122601 & 07-06-99 & $<0.035$ & $<0.017$ & $<0.02$ & $<0.42$ & $<0.002$ \\
\hline Buffalo Springs Lake & PS & 333205101414401 & 07-12-99 & $<.035$ & $<.017$ & .55 & $<.42$ & $<.002$ \\
\hline Cedar Creek Reservoir & PS & 295502096440201 & 07-19-99 & $<.035$ & $<.017$ & $<.02$ & $<.42$ & $<.002$ \\
\hline Choke Canyon Reservoir & $\mathrm{AC}$ & 8206890 & 07-07-99 & $<.035$ & $<.017$ & $<.02$ & $<.42$ & $<.002$ \\
\hline Donna Reservoir & PS & 260912098040601 & 07-13-99 & $<.035$ & $<.017$ & $<.02$ & $<.42$ & $<.002$ \\
\hline E.V. Spence Reservoir & PS & 315535100341701 & $07-15-99$ & $<.035$ & $<.017$ & .07 & $<.42$ & $<.002$ \\
\hline Falcon International Reservoir & $\mathrm{BC}$ & 263815099111901 & 07-07-99 & $<.035$ & $<.017$ & $<.02$ & $<.42$ & $<.002$ \\
\hline Fort Phantom Hill Reservoir & PS & 323632099410401 & $07-14-99$ & $<.035$ & $<.017$ & .16 & $<.42$ & $<.002$ \\
\hline Granger Lake & $\mathrm{AC}$ & 304132097200801 & 06-30-99 & $<.035$ & $<.017$ & .03 & $<.42$ & $<.002$ \\
\hline Grapevine Lake & $\mathrm{BC}$ & 325930097053801 & $07-26-99$ & $<.035$ & $<.017$ & .19 & $<.42$ & $<.002$ \\
\hline Greenbelt Lake & $\mathrm{AC}$ & 350000100534701 & 07-13-99 & $<.035$ & $<.017$ & $<.02$ & $<.42$ & $<.002$ \\
\hline Hubbard Creek Reservoir & P5 & 324913098581801 & $07-15-99$ & $<.035$ & $<.017$ & $<.02$ & $<.42$ & $<.002$ \\
\hline La Feria Reservoir & PS & 260822097493401 & 07-14-99 & $<.035$ & $<.017$ & $<.02$ & $<.42$ & $<.002$ \\
\hline Lake Anahuac & PS & 294624094411201 & $07-21-99$ & $<.035$ & $<.017$ & $<.02$ & $<.42$ & $<.002$ \\
\hline Lake Belton & $\mathrm{BC}$ & 310711097302201 & 06-30-99 & $<.035$ & $<.017$ & $<.02$ & $<.42$ & $<.002$ \\
\hline Lake Bonham & PS & 333858096083301 & $07-27-99$ & $<.035$ & $<.017$ & .04 & $<.42$ & $<.002$ \\
\hline Lake Bridgeport & Mid-lake & 331312097501801 & $07-21-99$ & $<.035$ & $<.017$ & E.03 & $<.42$ & $<.002$ \\
\hline Lake Brownwood & PS & 315026099005301 & 07-19-99 & $<.035$ & $<.017$ & $<.02$ & $<.42$ & $<.002$ \\
\hline Lake Houston & $\mathrm{BC}$ & 295702095091401 & 07-12-99 & $<.035$ & $<.017$ & .06 & $<.42$ & $<.002$ \\
\hline Lake J.B. Thomas & PS & 323510101085501 & $07-13-99$ & $<.035$ & $<.017$ & $<.02$ & $<.42$ & $<.002$ \\
\hline Lake Kickapoo & PS & 333913098480601 & 07-19-99 & $<.035$ & $<.017$ & $<.02$ & $<.42$ & $<.002$ \\
\hline Lake Limestone & $\mathrm{BC}$ & 312458096205101 & 07-08-99 & $<.035$ & $<.017$ & .05 & $<.42$ & $<.002$ \\
\hline Lake Meredith & $\mathrm{BC}$ & 354113101360101 & 07-13-99 & $<.035$ & $<.017$ & .07 & $<.42$ & $<.002$ \\
\hline Lake Mexia & PS & 313848096345001 & 07-07-99 & $<.035$ & $<.017$ & $<.02$ & $<.42$ & $<.002$ \\
\hline
\end{tabular}


Table 2. Concentrations of soluble pesticides in public water-supply reservoirs sampled in Texas, June-July 1999-Continued

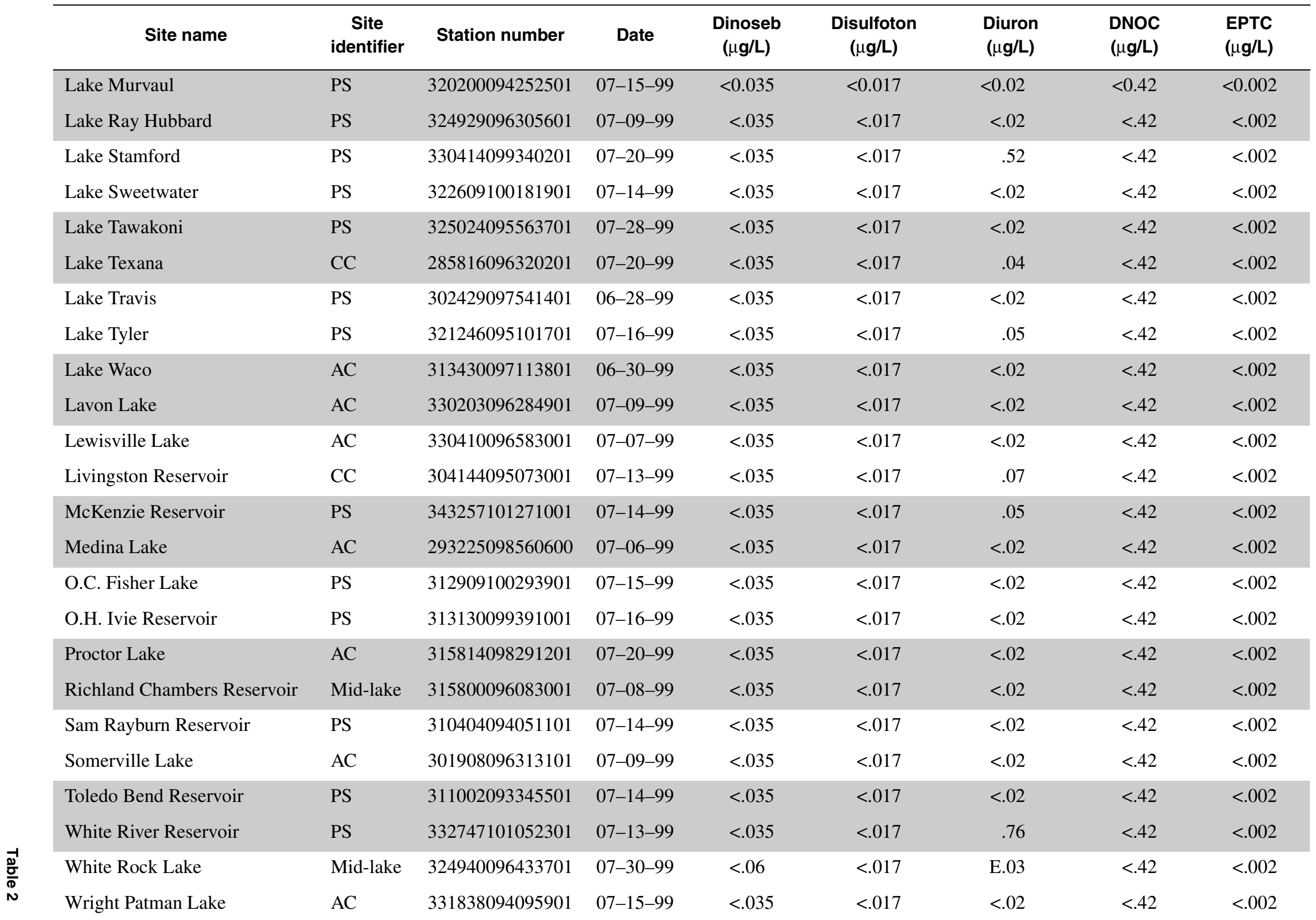


Table 2. Concentrations of soluble pesticides in public water-supply reservoirs sampled in Texas, June-July 1999-Continued

\begin{tabular}{|c|c|c|c|c|c|c|c|c|}
\hline Site name & $\begin{array}{c}\text { Site } \\
\text { identifier }\end{array}$ & Station number & Date & $\begin{array}{l}\text { Ethalfluralin } \\
\qquad(\mu \mathrm{g} / \mathrm{L})\end{array}$ & $\begin{array}{l}\text { Ethoprophos } \\
(\mu \mathrm{g} / \mathrm{L})\end{array}$ & $\begin{array}{c}\text { Fenuron } \\
(\mu \mathrm{g} / \mathrm{L})\end{array}$ & $\begin{array}{c}\text { Fonofos } \\
(\mu \mathrm{g} / \mathrm{L})\end{array}$ & $\begin{array}{l}\text { Fluometuron } \\
(\mu \mathrm{g} / \mathrm{L})\end{array}$ \\
\hline Aquilla Lake & $\mathrm{AC}$ & 315358097122601 & 07-06-99 & $<0.004$ & $<0.003$ & $<0.013$ & $<0.003$ & $<0.035$ \\
\hline Buffalo Springs Lake & PS & 333205101414401 & 07-12-99 & $<.004$ & $<.003$ & $<.14$ & $<.003$ & $<.035$ \\
\hline Cedar Creek Reservoir & PS & 295502096440201 & 07-19-99 & $<.004$ & $<.003$ & $<.013$ & $<.003$ & $<.035$ \\
\hline Choke Canyon Reservoir & $\mathrm{AC}$ & 8206890 & 07-07-99 & $<.004$ & $<.003$ & $<.013$ & $<.003$ & $<.035$ \\
\hline Donna Reservoir & PS & 260912098040601 & 07-13-99 & $<.004$ & $<.003$ & $<.013$ & $<.003$ & $<.035$ \\
\hline E.V. Spence Reservoir & PS & 315535100341701 & $07-15-99$ & $<.004$ & $<.003$ & .08 & $<.003$ & $<.035$ \\
\hline Falcon International Reservoir & $\mathrm{BC}$ & 263815099111901 & 07-07-99 & $<.004$ & $<.003$ & $<.013$ & $<.003$ & $<.035$ \\
\hline Fort Phantom Hill Reservoir & PS & 323632099410401 & $07-14-99$ & $<.004$ & $<.003$ & $<.013$ & $<.003$ & $<.035$ \\
\hline Granger Lake & $\mathrm{AC}$ & 304132097200801 & 06-30-99 & $<.004$ & $<.003$ & $<.013$ & $<.003$ & .06 \\
\hline Grapevine Lake & $\mathrm{BC}$ & 325930097053801 & $07-26-99$ & $<.004$ & $<.003$ & $<.013$ & $<.003$ & $<.035$ \\
\hline Greenbelt Lake & $\mathrm{AC}$ & 350000100534701 & 07-13-99 & $<.004$ & $<.003$ & $<.013$ & $<.003$ & $<.035$ \\
\hline Hubbard Creek Reservoir & P5 & 324913098581801 & $07-15-99$ & $<.004$ & $<.003$ & $<.013$ & $<.003$ & $<.035$ \\
\hline La Feria Reservoir & PS & 260822097493401 & 07-14-99 & $<.004$ & $<.003$ & $<.013$ & $<.003$ & $<.035$ \\
\hline Lake Anahuac & PS & 294624094411201 & $07-21-99$ & $<.004$ & $<.003$ & $<.013$ & $<.003$ & $<.035$ \\
\hline Lake Belton & $\mathrm{BC}$ & 310711097302201 & 06-30-99 & $<.004$ & $<.003$ & $<.013$ & $<.003$ & $<.035$ \\
\hline Lake Bonham & PS & 333858096083301 & $07-27-99$ & $<.004$ & $<.003$ & $<.013$ & $<.003$ & $<.035$ \\
\hline Lake Bridgeport & Mid-lake & 331312097501801 & $07-21-99$ & $<.004$ & $<.003$ & $<.013$ & $<.003$ & $<.035$ \\
\hline Lake Brownwood & PS & 315026099005301 & 07-19-99 & $<.004$ & $<.003$ & $<.013$ & $<.003$ & $<.035$ \\
\hline Lake Houston & $\mathrm{BC}$ & 295702095091401 & $07-12-99$ & $<.004$ & $<.003$ & $<.013$ & $<.003$ & $<.035$ \\
\hline Lake J.B. Thomas & PS & 323510101085501 & $07-13-99$ & $<.004$ & $<.003$ & $<.013$ & $<.003$ & $<.52$ \\
\hline Lake Kickapoo & PS & 333913098480601 & 07-19-99 & $<.004$ & $<.003$ & $<.04$ & $<.003$ & $<.035$ \\
\hline Lake Limestone & $\mathrm{BC}$ & 312458096205101 & 07-08-99 & $<.004$ & $<.003$ & $<.013$ & $<.003$ & $<.035$ \\
\hline Lake Meredith & $\mathrm{BC}$ & 354113101360101 & 07-13-99 & $<.004$ & $<.003$ & $<.013$ & $<.003$ & $<.035$ \\
\hline Lake Mexia & PS & 313848096345001 & 07-07-99 & $<.004$ & $<.003$ & $<.013$ & $<.003$ & $<.035$ \\
\hline
\end{tabular}


Table 2. Concentrations of soluble pesticides in public water-supply reservoirs sampled in Texas, June-July 1999-Continued

\begin{tabular}{|c|c|c|c|c|c|c|c|c|}
\hline Site name & $\begin{array}{c}\text { Site } \\
\text { identifier }\end{array}$ & Station number & Date & $\begin{array}{l}\text { Ethalfluralin } \\
\qquad(\mu \mathrm{g} / \mathrm{L})\end{array}$ & $\begin{array}{c}\text { Ethoprophos } \\
(\mu \mathrm{g} / \mathrm{L})\end{array}$ & $\begin{array}{c}\text { Fenuron } \\
(\mu \mathrm{g} / \mathrm{L})\end{array}$ & $\begin{array}{c}\text { Fonofos } \\
(\mu \mathrm{g} / \mathrm{L})\end{array}$ & $\begin{array}{l}\text { Fluometuron } \\
(\mu \mathrm{g} / \mathrm{L})\end{array}$ \\
\hline Lake Murvaul & PS & 320200094252501 & $07-15-99$ & $<0.004$ & $<0.003$ & $<0.013$ & $<0.003$ & $<0.035$ \\
\hline Lake Ray Hubbard & PS & 324929096305601 & 07-09-99 & $<.004$ & $<.003$ & $<.013$ & $<.003$ & $<.035$ \\
\hline Lake Stamford & PS & 330414099340201 & 07-20-99 & $<.004$ & $<.003$ & $<.013$ & $<.003$ & $<.035$ \\
\hline Lake Sweetwater & PS & 322609100181901 & 07-14-99 & $<.004$ & $<.003$ & $<.013$ & $<.003$ & $<.035$ \\
\hline Lake Tawakoni & PS & 325024095563701 & $07-28-99$ & $<.004$ & $<.003$ & $<.013$ & $<.003$ & $<.035$ \\
\hline Lake Travis & PS & 302429097541401 & 06-28-99 & $<.004$ & $<.003$ & $<.013$ & $<.003$ & $<.035$ \\
\hline Lake Tyler & PS & 321246095101701 & 07-16-99 & $<.004$ & $<.003$ & $<.013$ & $<.003$ & $<.19$ \\
\hline Lake Waco & $\mathrm{AC}$ & 313430097113801 & 06-30-99 & $<.004$ & $<.003$ & $<.013$ & $<.003$ & $<.035$ \\
\hline Lavon Lake & $\mathrm{AC}$ & 330203096284901 & 07-09-99 & $<.004$ & $<.003$ & $<.013$ & $<.003$ & $<.035$ \\
\hline Medina Lake & $\mathrm{AC}$ & 293225098560600 & 07-06-99 & $<.004$ & $<.003$ & $<.013$ & $<.003$ & $<.035$ \\
\hline O.C. Fisher Lake & PS & 312909100293901 & $07-15-99$ & $<.004$ & $<.003$ & $<.013$ & $<.003$ & $<.035$ \\
\hline O.H. Ivie Reservoir & PS & 313130099391001 & $07-16-99$ & $<.004$ & $<.003$ & $<.013$ & $<.003$ & $<.035$ \\
\hline Proctor Lake & $\mathrm{AC}$ & 315814098291201 & 07-20-99 & $<.004$ & $<.003$ & $<.12$ & $<.003$ & $<.035$ \\
\hline Richland Chambers Reservoir & Mid-lake & 315800096083001 & 07-08-99 & $<.004$ & $<.003$ & $<.013$ & $<.003$ & $<.035$ \\
\hline Sam Rayburn Reservoir & PS & 310404094051101 & 07-14-99 & $<.004$ & $<.003$ & $<.013$ & $<.003$ & $<.035$ \\
\hline Somerville Lake & $\mathrm{AC}$ & 301908096313101 & 07-09-99 & $<.004$ & $<.003$ & $<.11$ & $<.003$ & $<.035$ \\
\hline Toledo Bend Reservoir & PS & 311002093345501 & 07-14-99 & $<.004$ & $<.003$ & $<.013$ & $<.003$ & $<.035$ \\
\hline White River Reservoir & PS & 332747101052301 & 07-13-99 & $<.004$ & $<.003$ & $<.013$ & $<.003$ & .25 \\
\hline
\end{tabular}


Table 2. Concentrations of soluble pesticides in public water-supply reservoirs sampled in Texas, June-July 1999-Continued

\begin{tabular}{|c|c|c|c|c|c|c|c|c|c|}
\hline Site name & $\begin{array}{c}\text { Site } \\
\text { identifier }\end{array}$ & Station number & Date & $\begin{array}{l}\text { 3-Hydroxy- } \\
\text { carbofuran } \\
(\mu \mathrm{g} / \mathrm{L})\end{array}$ & $\begin{array}{c}\text { Lindane } \\
\text { ( } \mu \mathrm{g} / \mathrm{L})\end{array}$ & $\begin{array}{l}\text { Linuron } \\
\text { ( } \mu \mathrm{g} / \mathrm{L})\end{array}$ & $\begin{array}{c}\text { Malathion } \\
\quad(\mu \mathrm{g} / \mathrm{L})\end{array}$ & $\begin{array}{l}\text { MCPA } \\
(\mu g / L)\end{array}$ & $\begin{array}{l}\text { MCPB } \\
(\mu \mathrm{g} / \mathrm{L})\end{array}$ \\
\hline Aquilla Lake & $\mathrm{AC}$ & 315358097122601 & $07-06-99$ & $<0.014$ & $<0.004$ & $<0.002$ & $<0.005$ & $<0.17$ & $<0.14$ \\
\hline Buffalo Springs Lake & PS & 333205101414401 & $07-12-99$ & $<.17$ & $<.004$ & $<.002$ & $<.005$ & $<.17$ & $<.14$ \\
\hline Cedar Creek Reservoir & PS & 295502096440201 & 07-19-99 & $<.17$ & $<.004$ & $<.002$ & $<.005$ & $<.17$ & $<.14$ \\
\hline Choke Canyon Reservoir & $\mathrm{AC}$ & 8206890 & 07-07-99 & $<.014$ & $<.004$ & $<.002$ & $<.005$ & $<.17$ & $<.14$ \\
\hline Donna Reservoir & PS & 260912098040601 & 07-13-99 & $<.21$ & $<.004$ & $<.002$ & .014 & $<.17$ & $<.14$ \\
\hline E.V. Spence Reservoir & PS & 315535100341701 & $07-15-99$ & $<.014$ & $<.004$ & $<.002$ & $<.005$ & $<.17$ & $<.14$ \\
\hline Falcon International Reservoir & $\mathrm{BC}$ & 263815099111901 & 07-07-99 & $<.15$ & $<.004$ & $<.002$ & $<.005$ & $<.17$ & $<.14$ \\
\hline Fort Phantom Hill Reservoir & PS & 323632099410401 & 07-14-99 & $<.014$ & $<.004$ & $<.002$ & $<.005$ & $<.17$ & $<.14$ \\
\hline Granger Lake & $\mathrm{AC}$ & 304132097200801 & 06-30-99 & $<.014$ & $<.004$ & $<.002$ & $<.005$ & $<.17$ & $<.14$ \\
\hline Grapevine Lake & $\mathrm{BC}$ & 325930097053801 & $07-26-99$ & $<.014$ & $<.004$ & $<.002$ & $<.005$ & $<.17$ & $<.14$ \\
\hline Greenbelt Lake & $\mathrm{AC}$ & 350000100534701 & 07-13-99 & $<.014$ & $<.004$ & $<.002$ & $<.005$ & $<.17$ & $<.14$ \\
\hline Hubbard Creek Reservoir & P5 & 324913098581801 & $07-15-99$ & $<.014$ & $<.004$ & $<.002$ & $<.005$ & $<.17$ & $<.14$ \\
\hline La Feria Reservoir & PS & 260822097493401 & 07-14-99 & $<.014$ & $<.004$ & $<.002$ & $<.005$ & $<.17$ & $<.14$ \\
\hline Lake Anahuac & PS & 294624094411201 & $07-21-99$ & $<.03$ & $<.004$ & $<.002$ & $<.02$ & $<.17$ & $<.14$ \\
\hline Lake Belton & $\mathrm{BC}$ & 310711097302201 & 06-30-99 & $<.014$ & $<.004$ & $<.002$ & $<.005$ & $<.17$ & $<.14$ \\
\hline Lake Bonham & PS & 333858096083301 & 07-27-99 & $<.1$ & $<.004$ & $<.002$ & $<.005$ & $<.17$ & $<.14$ \\
\hline Lake Bridgeport & Mid-lake & 331312097501801 & $07-21-99$ & $<.014$ & $<.004$ & $<.002$ & $<.005$ & $<.17$ & $<.14$ \\
\hline Lake Brownwood & PS & 315026099005301 & 07-19-99 & $<.014$ & $<.004$ & $<.002$ & $<.005$ & $<.17$ & $<.14$ \\
\hline Lake Houston & $\mathrm{BC}$ & 295702095091401 & 07-12-99 & $<.014$ & $<.004$ & $<.002$ & $<.005$ & $<.17$ & $<.14$ \\
\hline Lake J.B. Thomas & PS & 323510101085501 & 07-13-99 & $<.014$ & $<.004$ & $<.002$ & .057 & $<.17$ & $<.14$ \\
\hline Lake Kickapoo & PS & 333913098480601 & 07-19-99 & $<.014$ & $<.004$ & $<.002$ & $<.005$ & $<.17$ & $<.14$ \\
\hline Lake Limestone & $\mathrm{BC}$ & 312458096205101 & 07-08-99 & $<.014$ & $<.004$ & $<.002$ & $<.005$ & $<.17$ & $<.14$ \\
\hline Lake Meredith & $\mathrm{BC}$ & 354113101360101 & 07-13-99 & $<.014$ & $<.004$ & $<.002$ & $<.005$ & $<.17$ & $<.14$ \\
\hline Lake Mexia & PS & 313848096345001 & 07-07-99 & $<.014$ & $<.004$ & $<.002$ & $<.005$ & $<.17$ & $<.14$ \\
\hline
\end{tabular}


Table 2. Concentrations of soluble pesticides in public water-supply reservoirs sampled in Texas, June-July 1999-Continued

\begin{tabular}{|c|c|c|c|c|c|c|c|c|c|}
\hline Site name & $\begin{array}{c}\text { Site } \\
\text { identifier }\end{array}$ & Station number & Date & $\begin{array}{c}\text { 3-Hydroxy- } \\
\text { carbofuran } \\
(\mu \mathrm{g} / \mathrm{L})\end{array}$ & $\begin{array}{c}\text { Lindane } \\
(\mu \mathbf{g} / L)\end{array}$ & $\begin{array}{c}\text { Linuron } \\
(\mu \mathbf{g} / L)\end{array}$ & $\begin{array}{c}\text { Malathion } \\
(\mu \mathrm{g} / \mathrm{L})\end{array}$ & $\begin{array}{l}\text { MCPA } \\
(\mu \mathrm{g} / \mathrm{L})\end{array}$ & $\begin{array}{l}\text { MCPB } \\
(\mu \mathrm{g} / \mathrm{L})\end{array}$ \\
\hline Lake Murvaul & PS & 320200094252501 & $07-15-99$ & $<0.014$ & $<0.004$ & $<0.002$ & $<0.005$ & $<0.17$ & $<0.14$ \\
\hline Lake Ray Hubbard & PS & 324929096305601 & 07-09-99 & $<.014$ & $<.004$ & $<.002$ & $<.005$ & $<.17$ & $<.14$ \\
\hline Lake Stamford & PS & 330414099340201 & 07-20-99 & $<.04$ & $<.004$ & $<.002$ & $<.005$ & $<.17$ & $<.14$ \\
\hline Lake Sweetwater & PS & 322609100181901 & 07-14-99 & $<.014$ & $<.004$ & $<.002$ & $<.005$ & $<.17$ & $<.14$ \\
\hline Lake Tawakoni & PS & 325024095563701 & 07-28-99 & $<.014$ & $<.004$ & $<.002$ & $<.005$ & $<.17$ & $<.14$ \\
\hline Lake Texana & $\mathrm{CC}$ & 285816096320201 & 07-20-99 & $<.14$ & $<.004$ & $<.002$ & .008 & $<.17$ & $<.14$ \\
\hline Lake Travis & PS & 302429097541401 & 06-28-99 & $<.014$ & $<.004$ & $<.002$ & $<.005$ & $<.17$ & $<.14$ \\
\hline Lake Tyler & PS & 321246095101701 & $07-16-99$ & $<.014$ & $<.004$ & $<.002$ & $<.005$ & $<.17$ & $<.14$ \\
\hline Lake Waco & $\mathrm{AC}$ & 313430097113801 & 06-30-99 & $<.014$ & $<.004$ & $<.002$ & $<.005$ & $<.17$ & $<.14$ \\
\hline Lavon Lake & $\mathrm{AC}$ & 330203096284901 & 07-09-99 & $<.014$ & $<.004$ & $<.002$ & $<.005$ & $<.17$ & $<.14$ \\
\hline Lewisville Lake & $\mathrm{AC}$ & 330410096583001 & 07-07-99 & $<.014$ & $<.004$ & $<.002$ & $<.005$ & $<.17$ & $<.14$ \\
\hline Livingston Reservoir & $\mathrm{CC}$ & 304144095073001 & 07-13-99 & $<.17$ & $<.004$ & $<.002$ & $<.005$ & $<.17$ & $<.14$ \\
\hline McKenzie Reservoir & PS & 343257101271001 & 07-14-99 & $<1.91$ & $<.004$ & $<.002$ & $<.005$ & $<.17$ & $<.14$ \\
\hline Medina Lake & $\mathrm{AC}$ & 293225098560600 & 07-06-99 & $<.014$ & $<.004$ & $<.002$ & $<.005$ & $<.17$ & $<.14$ \\
\hline O.C. Fisher Lake & PS & 312909100293901 & $07-15-99$ & $<.014$ & $<.004$ & $<.002$ & $<.005$ & $<.17$ & $<.14$ \\
\hline O.H. Ivie Reservoir & PS & 313130099391001 & $07-16-99$ & $<.014$ & $<.004$ & $<.002$ & $<.005$ & $<.17$ & $<.14$ \\
\hline Proctor Lake & $\mathrm{AC}$ & 315814098291201 & 07-20-99 & $<.16$ & $<.004$ & $<.002$ & $<.005$ & $<.17$ & $<.14$ \\
\hline Richland Chambers Reservoir & Mid-lake & 315800096083001 & 07-08-99 & $<.014$ & $<.004$ & $<.002$ & $<.005$ & $<.17$ & $<.14$ \\
\hline Sam Rayburn Reservoir & PS & 310404094051101 & 07-14-99 & $<.014$ & $<.004$ & $<.002$ & $<.005$ & $<.17$ & $<.14$ \\
\hline Somerville Lake & $\mathrm{AC}$ & 301908096313101 & 07-09-99 & $<.11$ & $<.004$ & $<.002$ & $<.005$ & $<.17$ & $<.14$ \\
\hline Toledo Bend Reservoir & PS & 311002093345501 & 07-14-99 & $<.014$ & $<.004$ & $<.002$ & $<.005$ & $<.17$ & $<.14$ \\
\hline White River Reservoir & PS & 332747101052301 & 07-13-99 & $<.18$ & $<.004$ & $<.002$ & $<.005$ & $<.17$ & $<.14$ \\
\hline White Rock Lake & Mid-lake & 324940096433701 & 07-30-99 & $<.11$ & $<.004$ & $<.002$ & $<.005$ & $<.17$ & $<.13$ \\
\hline Wright Patman Lake & $\mathrm{AC}$ & 331838094095901 & $07-15-99$ & $<.014$ & $<.004$ & $<.002$ & $<.005$ & $<.17$ & $<.14$ \\
\hline
\end{tabular}


Table 2. Concentrations of soluble pesticides in public water-supply reservoirs sampled in Texas, June-July 1999—Continued

\begin{tabular}{|c|c|c|c|c|c|c|c|}
\hline Site name & $\begin{array}{c}\text { Site } \\
\text { identifier }\end{array}$ & Station number & Date & $\begin{array}{c}\text { Methiocarb } \\
(\mu \mathrm{g} / \mathrm{L})\end{array}$ & $\begin{array}{l}\text { Methomyl } \\
(\mu \mathrm{g} / \mathrm{L})\end{array}$ & $\begin{array}{c}\text { Metolachlor } \\
(\mu \mathrm{g} / \mathrm{L})\end{array}$ & $\begin{array}{l}\text { Metribuzin } \\
(\mu \mathrm{g} / \mathrm{L})\end{array}$ \\
\hline Aquilla Lake & $\mathrm{AC}$ & 315358097122601 & 07-06-99 & $<0.026$ & $<0.017$ & 0.287 & $<0.004$ \\
\hline Buffalo Springs Lake & PS & 333205101414401 & 07-12-99 & $<.026$ & $<.38$ & .011 & $<.004$ \\
\hline Cedar Creek Reservoir & PS & 295502096440201 & 07-19-99 & $<.026$ & $<.017$ & $<.006$ & $<.004$ \\
\hline Choke Canyon Reservoir & $\mathrm{AC}$ & 8206890 & 07-07-99 & $<.026$ & $<.27$ & .004 & $<.004$ \\
\hline Donna Reservoir & PS & 260912098040601 & 07-13-99 & $<.026$ & $<.017$ & $<.002$ & $<.004$ \\
\hline E.V. Spence Reservoir & PS & 315535100341701 & 07-15-99 & $<.026$ & $<.8$ & $<.002$ & $<.004$ \\
\hline Falcon International Reservoir & $\mathrm{BC}$ & 263815099111901 & 07-07-99 & $<.026$ & $<.35$ & $<.002$ & $<.004$ \\
\hline Fort Phantom Hill Reservoir & PS & 323632099410401 & 07-14-99 & $<.026$ & $<.37$ & $<.002$ & $<.004$ \\
\hline Granger Lake & $\mathrm{AC}$ & 304132097200801 & 06-30-99 & $<.026$ & $<.14$ & .230 & $<.004$ \\
\hline Grapevine Lake & $\mathrm{BC}$ & 325930097053801 & 07-26-99 & $<.026$ & $<.59$ & .015 & $<.004$ \\
\hline Greenbelt Lake & $\mathrm{AC}$ & 350000100534701 & 07-13-99 & $<.026$ & $<1.01$ & .005 & $<.004$ \\
\hline Hubbard Creek Reservoir & P5 & 324913098581801 & 07-15-99 & $<.026$ & $<.017$ & $<.002$ & $<.004$ \\
\hline La Feria Reservoir & PS & 260822097493401 & 07-14-99 & $<.026$ & $<2.19$ & $<.002$ & $<.004$ \\
\hline Lake Anahuac & PS & 294624094411201 & 07-21-99 & $<.026$ & $<.017$ & .037 & .017 \\
\hline Lake Belton & $\mathrm{BC}$ & 310711097302201 & 06-30-99 & $<.026$ & $<.017$ & .031 & $<.004$ \\
\hline Lake Bonham & PS & 333858096083301 & 07-27-99 & $<.026$ & $<.017$ & $<.0075$ & $<.004$ \\
\hline Lake Bridgeport & Mid-lake & 331312097501801 & 07-21-99 & $<.026$ & $<.017$ & E.004 & $<.004$ \\
\hline Lake Brownwood & PS & 315026099005301 & 07-19-99 & $<.026$ & $<.017$ & .006 & $<.004$ \\
\hline Lake Houston & $\mathrm{BC}$ & 295702095091401 & 07-12-99 & $<.026$ & $<.017$ & .012 & $<.004$ \\
\hline Lake J.B. Thomas & PS & 323510101085501 & 07-13-99 & $<.026$ & $<.017$ & $<.002$ & $<.004$ \\
\hline Lake Kickapoo & PS & 333913098480601 & 07-19-99 & $<.026$ & $<.017$ & $<.002$ & $<.004$ \\
\hline Lake Limestone & $\mathrm{BC}$ & 312458096205101 & 07-08-99 & $<.026$ & $<.11$ & .006 & $<.004$ \\
\hline Lake Meredith & $\mathrm{BC}$ & 354113101360101 & 07-13-99 & $<.026$ & $<.58$ & .009 & $<.004$ \\
\hline Lake Mexia & PS & 313848096345001 & 07-07-99 & $<.026$ & $<.43$ & .007 & $<.004$ \\
\hline
\end{tabular}


Table 2. Concentrations of soluble pesticides in public water-supply reservoirs sampled in Texas, June-July 1999—Continued

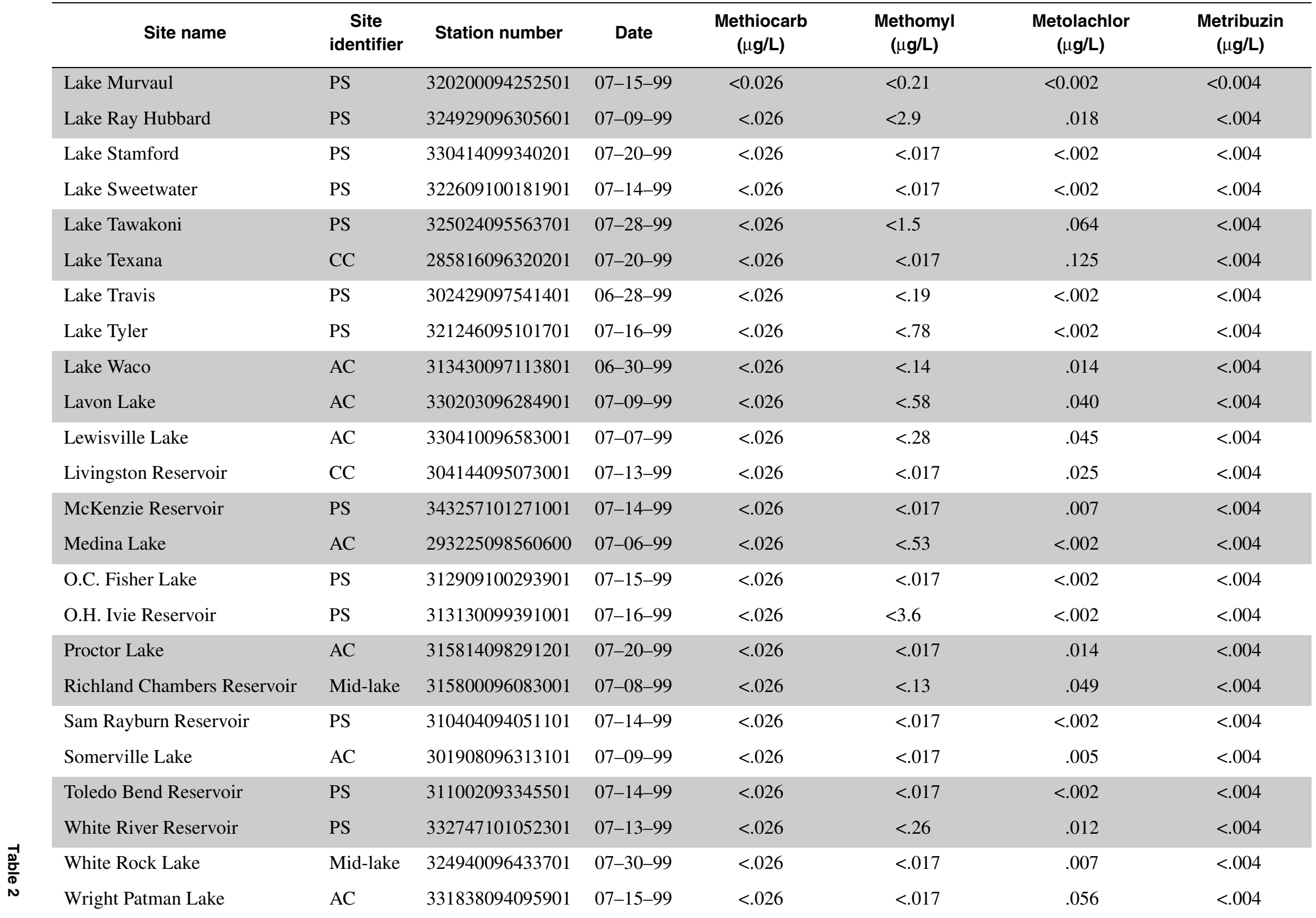


Table 2. Concentrations of soluble pesticides in public water-supply reservoirs sampled in Texas, June-July 1999-Continued

\begin{tabular}{|c|c|c|c|c|c|c|c|c|}
\hline Site name & $\begin{array}{c}\text { Site } \\
\text { identifier }\end{array}$ & Station number & Date & $\begin{array}{c}\text { Molinate } \\
(\mu \mathrm{g} / \mathrm{L})\end{array}$ & $\begin{array}{c}\text { Napropamide } \\
(\mu g / L)\end{array}$ & $\begin{array}{c}\text { Neburon } \\
(\mu \mathrm{g} / \mathrm{L})\end{array}$ & $\begin{array}{l}\text { Norflurazon } \\
(\mu \mathrm{g} / \mathrm{L})\end{array}$ & $\begin{array}{c}\text { Oryzalin } \\
(\mu \mathrm{g} / \mathrm{L})\end{array}$ \\
\hline Aquilla Lake & $\mathrm{AC}$ & 315358097122601 & 07-06-99 & $<0.004$ & $<0.003$ & $<0.015$ & $<0.024$ & $<0.71$ \\
\hline Buffalo Springs Lake & PS & 333205101414401 & $07-12-99$ & $<.004$ & $<.003$ & $<.015$ & $<.024$ & $<.41$ \\
\hline Cedar Creek Reservoir & PS & 295502096440201 & 07-19-99 & $<.004$ & $<.003$ & $<.015$ & $<.024$ & $<.38$ \\
\hline Choke Canyon Reservoir & $\mathrm{AC}$ & 8206890 & 07-07-99 & $<.004$ & $<.003$ & $<.015$ & $<.024$ & $<.31$ \\
\hline Donna Reservoir & PS & 260912098040601 & 07-13-99 & $<.004$ & $<.003$ & $<.015$ & $<.024$ & $<.46$ \\
\hline E.V. Spence Reservoir & PS & 315535100341701 & 07-15-99 & $<.004$ & $<.003$ & $<.015$ & $<.024$ & $<.31$ \\
\hline Falcon International Reservoir & $\mathrm{BC}$ & 263815099111901 & 07-07-99 & $<.004$ & $<.003$ & $<.015$ & $<.024$ & $<.31$ \\
\hline Fort Phantom Hill Reservoir & PS & 323632099410401 & 07-14-99 & $<.004$ & $<.003$ & $<.015$ & $<.024$ & $<.31$ \\
\hline Granger Lake & $\mathrm{AC}$ & 304132097200801 & 06-30-99 & $<.004$ & $<.003$ & $<.015$ & $<.024$ & $<.47$ \\
\hline Grapevine Lake & $\mathrm{BC}$ & 325930097053801 & 07-26-99 & $<.004$ & $<.003$ & $<.015$ & $<.024$ & $<.4$ \\
\hline Greenbelt Lake & $\mathrm{AC}$ & 350000100534701 & 07-13-99 & $<.004$ & $<.003$ & $<.015$ & $<.024$ & $<.46$ \\
\hline Hubbard Creek Reservoir & P5 & 324913098581801 & $07-15-99$ & $<.004$ & $<.003$ & $<.015$ & $<.024$ & $<.49$ \\
\hline La Feria Reservoir & PS & 260822097493401 & 07-14-99 & $<.004$ & $<.003$ & $<.015$ & $<.024$ & $<.5$ \\
\hline Lake Anahuac & PS & 294624094411201 & $07-21-99$ & $<.004$ & $<.003$ & $<.015$ & $<.024$ & $<.42$ \\
\hline Lake Belton & $\mathrm{BC}$ & 310711097302201 & 06-30-99 & $<.004$ & $<.003$ & $<.015$ & $<.024$ & $<.46$ \\
\hline Lake Bonham & PS & 333858096083301 & $07-27-99$ & $<.004$ & $<.003$ & $<.015$ & $<.024$ & $<.31$ \\
\hline Lake Bridgeport & Mid-lake & 331312097501801 & $07-21-99$ & $<.004$ & $<.003$ & $<.015$ & $<.024$ & $<.32$ \\
\hline Lake Brownwood & PS & 315026099005301 & 07-19-99 & $<.004$ & $<.003$ & $<.015$ & $<.024$ & $<.36$ \\
\hline Lake Houston & $\mathrm{BC}$ & 295702095091401 & 07-12-99 & $<.004$ & $<.003$ & $<.015$ & $<.024$ & $<.31$ \\
\hline Lake J.B. Thomas & PS & 323510101085501 & 07-13-99 & $<.004$ & $<.003$ & $<.015$ & $<.024$ & $<.36$ \\
\hline Lake Kickapoo & PS & 333913098480601 & 07-19-99 & $<.004$ & $<.003$ & $<.015$ & $<.024$ & $<.44$ \\
\hline Lake Limestone & $\mathrm{BC}$ & 312458096205101 & 07-08-99 & $<.004$ & $<.003$ & $<.015$ & $<.024$ & $<.31$ \\
\hline Lake Meredith & $\mathrm{BC}$ & 354113101360101 & 07-13-99 & $<.004$ & $<.003$ & $<.015$ & $<.024$ & $<.41$ \\
\hline Lake Mexia & PS & 313848096345001 & 07-07-99 & $<.004$ & $<.003$ & $<.015$ & $<.024$ & $<.46$ \\
\hline
\end{tabular}


Table 2. Concentrations of soluble pesticides in public water-supply reservoirs sampled in Texas, June-July 1999-Continued

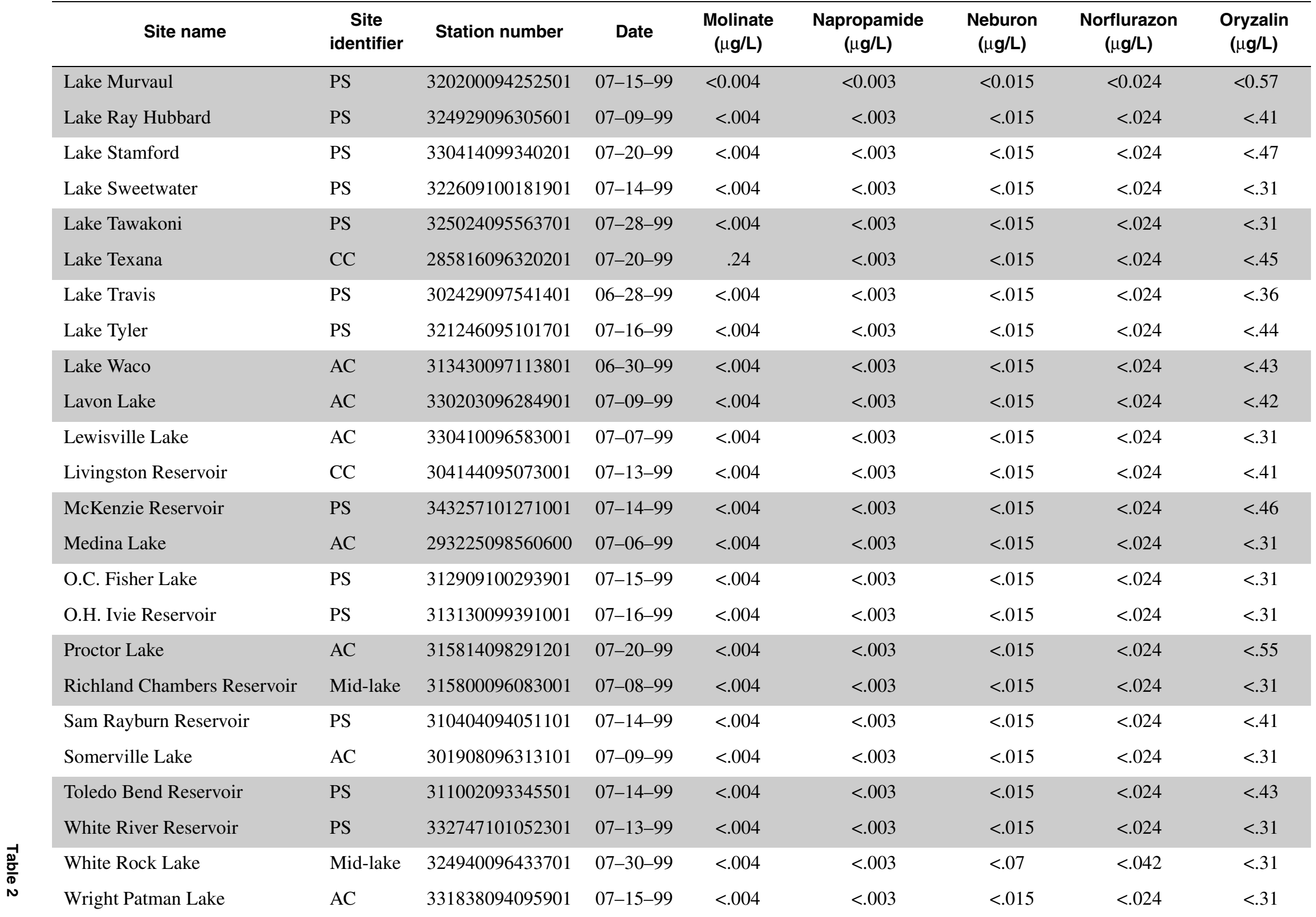


Table 2. Concentrations of soluble pesticides in public water-supply reservoirs sampled in Texas, June-July 1999—Continued

\begin{tabular}{|c|c|c|c|c|c|c|c|c|}
\hline Site name & $\begin{array}{c}\text { Site } \\
\text { identifier }\end{array}$ & Station number & Date & $\begin{array}{c}\text { Oxamyl } \\
\text { ( } \mu \mathrm{g} / \mathrm{L})\end{array}$ & $\begin{array}{c}\text { Parathion } \\
(\mu \mathrm{g} / \mathrm{L})\end{array}$ & $\begin{array}{c}\text { Parathion- } \\
\text { methyl } \\
(\mu \mathrm{g} / \mathrm{L})\end{array}$ & $\begin{array}{c}\text { Pebulate } \\
\text { ( } \mu \mathrm{g} / \mathrm{L})\end{array}$ & $\begin{array}{l}\text { Pendimethalin } \\
(\mu \mathrm{g} / \mathrm{L})\end{array}$ \\
\hline Aquilla Lake & $\mathrm{AC}$ & 315358097122601 & $07-06-99$ & $<0.018$ & $<0.004$ & $<0.006$ & $<0.004$ & $<0.004$ \\
\hline Buffalo Springs Lake & PS & 333205101414401 & $07-12-99$ & $<.018$ & $<.004$ & $<.006$ & $<.004$ & $<.004$ \\
\hline Cedar Creek Reservoir & PS & 295502096440201 & 07-19-99 & $<.018$ & $<.004$ & $<.006$ & $<.004$ & $<.004$ \\
\hline Choke Canyon Reservoir & $\mathrm{AC}$ & 8206890 & 07-07-99 & $<.018$ & $<.004$ & $<.006$ & $<.004$ & $<.004$ \\
\hline Donna Reservoir & PS & 260912098040601 & 07-13-99 & $<.018$ & $<.004$ & $<.01$ & $<.004$ & $<.004$ \\
\hline E.V. Spence Reservoir & PS & 315535100341701 & $07-15-99$ & $<.018$ & $<.004$ & $<.006$ & $<.004$ & $<.004$ \\
\hline Falcon International Reservoir & $\mathrm{BC}$ & 263815099111901 & 07-07-99 & $<.018$ & $<.004$ & $<.006$ & $<.004$ & $<.004$ \\
\hline Fort Phantom Hill Reservoir & PS & 323632099410401 & 07-14-99 & $<.018$ & $<.004$ & $<.006$ & $<.004$ & $<.004$ \\
\hline Granger Lake & $\mathrm{AC}$ & 304132097200801 & 06-30-99 & $<.018$ & $<.004$ & $<.006$ & $<.004$ & $<.004$ \\
\hline Grapevine Lake & $\mathrm{BC}$ & 325930097053801 & 07-26-99 & $<.018$ & $<.004$ & $<.006$ & $<.004$ & $<.004$ \\
\hline Greenbelt Lake & $\mathrm{AC}$ & 350000100534701 & 07-13-99 & $<.018$ & $<.004$ & $<.006$ & $<.004$ & $<.004$ \\
\hline Hubbard Creek Reservoir & P5 & 324913098581801 & $07-15-99$ & $<.018$ & $<.004$ & $<.006$ & $<.004$ & $<.004$ \\
\hline La Feria Reservoir & PS & 260822097493401 & 07-14-99 & $<.018$ & $<.004$ & .034 & $<.004$ & $<.004$ \\
\hline Lake Anahuac & PS & 294624094411201 & $07-21-99$ & $<.018$ & $<.004$ & $<.006$ & $<.004$ & $<.004$ \\
\hline Lake Belton & $\mathrm{BC}$ & 310711097302201 & 06-30-99 & $<.018$ & $<.004$ & $<.006$ & $<.004$ & $<.004$ \\
\hline Lake Bonham & PS & 333858096083301 & 07-27-99 & $<.018$ & $<.004$ & $<.006$ & $<.004$ & $<.004$ \\
\hline Lake Bridgeport & Mid-lake & 331312097501801 & $07-21-99$ & $<.018$ & $<.004$ & $<.006$ & $<.004$ & $<.004$ \\
\hline Lake Brownwood & PS & 315026099005301 & 07-19-99 & $<.018$ & $<.004$ & $<.006$ & $<.004$ & $<.004$ \\
\hline Lake Houston & $\mathrm{BC}$ & 295702095091401 & 07-12-99 & $<.018$ & $<.004$ & $<.006$ & $<.004$ & $<.004$ \\
\hline Lake J.B. Thomas & PS & 323510101085501 & 07-13-99 & $<.018$ & $<.004$ & $<.006$ & $<.004$ & $<.004$ \\
\hline Lake Kickapoo & PS & 333913098480601 & 07-19-99 & $<.018$ & $<.004$ & $<.006$ & $<.004$ & $<.004$ \\
\hline Lake Limestone & $\mathrm{BC}$ & 312458096205101 & 07-08-99 & $<.018$ & $<.004$ & $<.006$ & $<.004$ & $<.004$ \\
\hline Lake Meredith & $\mathrm{BC}$ & 354113101360101 & 07-13-99 & $<.018$ & $<.004$ & $<.006$ & $<.004$ & $<.004$ \\
\hline Lake Mexia & PS & 313848096345001 & 07-07-99 & $<.018$ & $<.004$ & $<.006$ & $<.004$ & $<.004$ \\
\hline
\end{tabular}


Table 2. Concentrations of soluble pesticides in public water-supply reservoirs sampled in Texas, June-July 1999—Continued

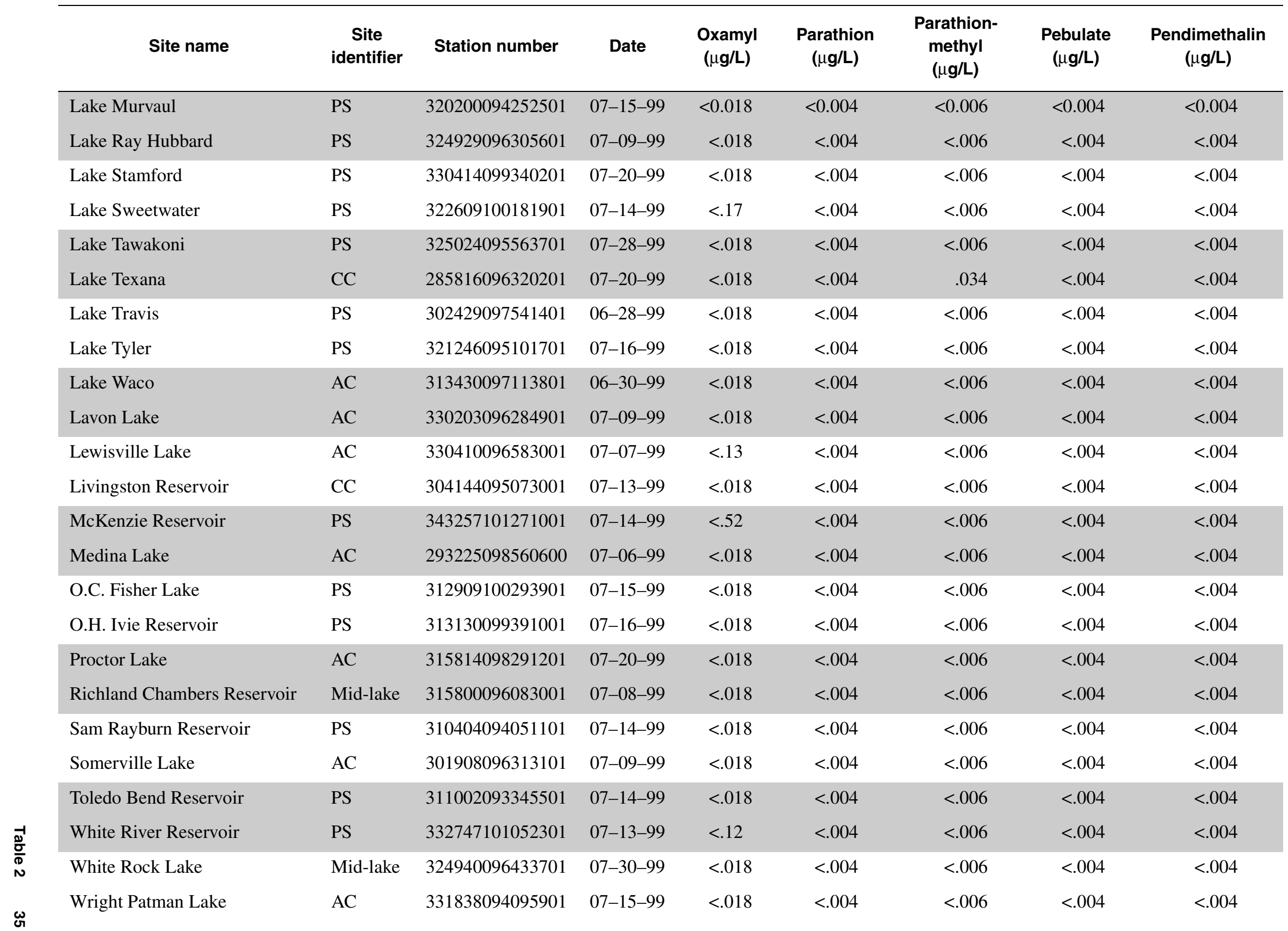


Table 2. Concentrations of soluble pesticides in public water-supply reservoirs sampled in Texas, June-July 1999—Continued

\begin{tabular}{|c|c|c|c|c|c|c|c|c|}
\hline Site name & $\begin{array}{c}\text { Site } \\
\text { identifier }\end{array}$ & Station number & Date & $\begin{array}{c}\text { cis- } \\
\text { Permethrin } \\
(\mu \mathrm{g} / \mathrm{L})\end{array}$ & $\begin{array}{c}\text { Phorate } \\
(\mu \mathrm{g} / \mathrm{L})\end{array}$ & $\begin{array}{c}\text { Picloram } \\
(\mu \mathrm{g} / \mathrm{L})\end{array}$ & $\begin{array}{c}p, p^{\prime}-\mathrm{DDE} \\
(\mu \mathrm{g} / \mathrm{L})\end{array}$ & $\begin{array}{l}\text { Prometon } \\
\quad(\mu \mathrm{g} / \mathrm{L})\end{array}$ \\
\hline Aquilla Lake & $\mathrm{AC}$ & 315358097122601 & $07-06-99$ & $<0.005$ & $<0.002$ & $<0.05$ & $<0.006$ & $<0.018$ \\
\hline Buffalo Springs Lake & PS & 333205101414401 & 07-12-99 & $<.005$ & $<.002$ & $<.05$ & $<.006$ & .142 \\
\hline Cedar Creek Reservoir & PS & 295502096440201 & 07-19-99 & $<.005$ & $<.002$ & $<.05$ & $<.006$ & E.012 \\
\hline Choke Canyon Reservoir & $\mathrm{AC}$ & 8206890 & 07-07-99 & $<.005$ & $<.002$ & $<.05$ & $<.006$ & $<.018$ \\
\hline Donna Reservoir & PS & 260912098040601 & 07-13-99 & $<.005$ & $<.002$ & $<.05$ & $<.006$ & $<.018$ \\
\hline E.V. Spence Reservoir & PS & 315535100341701 & 07-15-99 & $<.005$ & $<.002$ & $<.05$ & $<.006$ & E.016 \\
\hline Falcon International Reservoir & $\mathrm{BC}$ & 263815099111901 & 07-07-99 & $<.005$ & $<.002$ & $<.05$ & $<.006$ & $<.018$ \\
\hline Fort Phantom Hill Reservoir & PS & 323632099410401 & 07-14-99 & $<.005$ & $<.002$ & $<.05$ & $<.006$ & .052 \\
\hline Granger Lake & $\mathrm{AC}$ & 304132097200801 & 06-30-99 & $<.005$ & $<.002$ & $<.05$ & $<.006$ & $<.018$ \\
\hline Grapevine Lake & $\mathrm{BC}$ & 325930097053801 & 07-26-99 & $<.005$ & $<.002$ & $<.05$ & $<.006$ & $<.018$ \\
\hline Greenbelt Lake & $\mathrm{AC}$ & 350000100534701 & 07-13-99 & $<.005$ & $<.002$ & $<.05$ & $<.006$ & E.003 \\
\hline Hubbard Creek Reservoir & P5 & 324913098581801 & 07-15-99 & $<.005$ & $<.002$ & $<.05$ & $<.006$ & E.007 \\
\hline La Feria Reservoir & PS & 260822097493401 & 07-14-99 & $<.005$ & $<.002$ & $<.05$ & $<.006$ & $<.018$ \\
\hline Lake Anahuac & PS & 294624094411201 & $07-21-99$ & $<.005$ & $<.002$ & $<.05$ & $<.006$ & $<.018$ \\
\hline Lake Belton & $\mathrm{BC}$ & 310711097302201 & 06-30-99 & $<.005$ & $<.002$ & $<.05$ & $<.006$ & $<.018$ \\
\hline Lake Bonham & PS & 333858096083301 & 07-27-99 & $<.005$ & $<.002$ & $<.05$ & $<.006$ & $<.018$ \\
\hline Lake Bridgeport & Mid-lake & 331312097501801 & 07-21-99 & $<.005$ & $<.002$ & $<.05$ & $<.006$ & E.002 \\
\hline Lake Brownwood & PS & 315026099005301 & 07-19-99 & $<.005$ & $<.002$ & $<.05$ & $<.006$ & .019 \\
\hline Lake Houston & $\mathrm{BC}$ & 295702095091401 & 07-12-99 & $<.005$ & $<.002$ & $<.05$ & $<.006$ & E.008 \\
\hline Lake J.B. Thomas & PS & 323510101085501 & 07-13-99 & $<.005$ & $<.002$ & $<.05$ & $<.006$ & $<.018$ \\
\hline Lake Kickapoo & PS & 333913098480601 & 07-19-99 & $<.005$ & $<.002$ & $<.05$ & $<.006$ & $<.018$ \\
\hline Lake Limestone & $\mathrm{BC}$ & 312458096205101 & 07-08-99 & $<.005$ & $<.002$ & $<.05$ & $<.006$ & $<.018$ \\
\hline Lake Meredith & $\mathrm{BC}$ & 354113101360101 & 07-13-99 & $<.005$ & $<.002$ & $<.05$ & $<.006$ & .021 \\
\hline Lake Mexia & PS & 313848096345001 & 07-07-99 & $<.005$ & $<.002$ & $<.05$ & $<.006$ & $<.018$ \\
\hline
\end{tabular}


Table 2. Concentrations of soluble pesticides in public water-supply reservoirs sampled in Texas, June-July 1999—Continued

\begin{tabular}{|c|c|c|c|c|c|c|c|c|}
\hline Site name & $\begin{array}{c}\text { Site } \\
\text { identifier }\end{array}$ & Station number & Date & $\begin{array}{c}\text { cis- } \\
\text { Permethrin } \\
(\mu \mathrm{g} / \mathrm{L})\end{array}$ & $\begin{array}{c}\text { Phorate } \\
(\mu \mathrm{g} / \mathrm{L})\end{array}$ & $\begin{array}{c}\text { Picloram } \\
\text { ( } \mu \mathrm{g} / \mathrm{L})\end{array}$ & $\begin{array}{c}p, p^{\prime}-\mathrm{DDE} \\
(\mu \mathrm{g} / \mathrm{L})\end{array}$ & $\begin{array}{l}\text { Prometon } \\
(\mu \mathrm{g} / \mathrm{L})\end{array}$ \\
\hline Lake Murvaul & PS & 320200094252501 & $07-15-99$ & $<0.005$ & $<0.002$ & $<0.05$ & $<0.006$ & $<0.018$ \\
\hline Lake Stamford & PS & 330414099340201 & 07-20-99 & $<.005$ & $<.002$ & $<.05$ & $<.006$ & E.010 \\
\hline Lake Sweetwater & PS & 322609100181901 & 07-14-99 & $<.005$ & $<.002$ & $<.05$ & $<.006$ & $<.018$ \\
\hline Lake Texana & $\mathrm{CC}$ & 285816096320201 & $07-20-99$ & $<.005$ & $<.002$ & $<.05$ & $<.006$ & $<.018$ \\
\hline Lake Travis & PS & 302429097541401 & 06-28-99 & $<.005$ & $<.002$ & $<.05$ & $<.006$ & $<.018$ \\
\hline Lake Tyler & PS & 321246095101701 & 07-16-99 & $<.005$ & $<.002$ & $<.05$ & $<.006$ & $<.018$ \\
\hline Lake Waco & $\mathrm{AC}$ & 313430097113801 & 06-30-99 & $<.005$ & $<.002$ & $<.05$ & $<.006$ & $<.018$ \\
\hline Lavon Lake & $\mathrm{AC}$ & 330203096284901 & 07-09-99 & $<.005$ & $<.002$ & $<.05$ & $<.006$ & E.004 \\
\hline Medina Lake & $\mathrm{AC}$ & 293225098560600 & 07-06-99 & $<.005$ & $<.002$ & $<.05$ & $<.006$ & $<.018$ \\
\hline O.C. Fisher Lake & PS & 312909100293901 & $07-15-99$ & $<.005$ & $<.002$ & $<.05$ & $<.006$ & $<.018$ \\
\hline O.H. Ivie Reservoir & PS & 313130099391001 & 07-16-99 & $<.005$ & $<.002$ & $<.05$ & $<.006$ & $<.018$ \\
\hline Proctor Lake & $\mathrm{AC}$ & 315814098291201 & 07-20-99 & $<.005$ & $<.002$ & $<.05$ & $<.006$ & $<.018$ \\
\hline Richland Chambers Reservoir & Mid-lake & 315800096083001 & 07-08-99 & $<.005$ & $<.002$ & $<.05$ & $<.006$ & $<.018$ \\
\hline Sam Rayburn Reservoir & PS & 310404094051101 & 07-14-99 & $<.005$ & $<.002$ & $<.05$ & $<.006$ & $<.018$ \\
\hline Somerville Lake & $\mathrm{AC}$ & 301908096313101 & 07-09-99 & $<.005$ & $<.002$ & $<.05$ & $<.006$ & $<.018$ \\
\hline Toledo Bend Reservoir & PS & 311002093345501 & 07-14-99 & $<.005$ & $<.002$ & $<.05$ & $<.006$ & E.004 \\
\hline
\end{tabular}


Table 2. Concentrations of soluble pesticides in public water-supply reservoirs sampled in Texas, June-July 1999-Continued

\begin{tabular}{|c|c|c|c|c|c|c|c|c|}
\hline Site name & $\begin{array}{c}\text { Site } \\
\text { identifier }\end{array}$ & Station number & Date & $\begin{array}{c}\text { Pronamide } \\
(\mu \mathrm{g} / \mathrm{L})\end{array}$ & $\begin{array}{c}\text { Propachlor } \\
(\mu g / L)\end{array}$ & $\begin{array}{c}\text { Propanil } \\
(\mu \mathrm{g} / \mathrm{L})\end{array}$ & $\begin{array}{c}\text { Propargite } \\
(\mu \mathrm{g} / \mathrm{L})\end{array}$ & $\begin{array}{c}\text { Propham } \\
(\mu \mathrm{g} / \mathrm{L})\end{array}$ \\
\hline Aquilla Lake & $\mathrm{AC}$ & 315358097122601 & 07-06-99 & $<0.003$ & $<0.007$ & $<0.004$ & $<0.013$ & $<0.035$ \\
\hline Buffalo Springs Lake & PS & 333205101414401 & $07-12-99$ & $<.003$ & $<.007$ & $<.004$ & $<.03$ & $<.035$ \\
\hline Cedar Creek Reservoir & PS & 295502096440201 & 07-19-99 & $<.003$ & $<.007$ & $<.004$ & -- & $<.035$ \\
\hline Choke Canyon Reservoir & $\mathrm{AC}$ & 8206890 & 07-07-99 & $<.003$ & $<.007$ & $<.004$ & $<.013$ & $<.035$ \\
\hline Donna Reservoir & PS & 260912098040601 & $07-13-99$ & $<.003$ & $<.007$ & $<.004$ & $<.013$ & $<.035$ \\
\hline E.V. Spence Reservoir & PS & 315535100341701 & $07-15-99$ & $<.003$ & $<.007$ & $<.004$ & $<.013$ & $<.035$ \\
\hline Falcon International Reservoir & $\mathrm{BC}$ & 263815099111901 & 07-07-99 & $<.003$ & $<.007$ & $<.004$ & $<.013$ & $<.035$ \\
\hline Fort Phantom Hill Reservoir & PS & 323632099410401 & $07-14-99$ & $<.003$ & $<.007$ & $<.004$ & $<.013$ & $<.035$ \\
\hline Granger Lake & $\mathrm{AC}$ & 304132097200801 & 06-30-99 & $<.003$ & $<.007$ & $<.004$ & $<.013$ & $<.035$ \\
\hline Grapevine Lake & $\mathrm{BC}$ & 325930097053801 & $07-26-99$ & $<.003$ & $<.007$ & $<.004$ & $<.013$ & $<.035$ \\
\hline Greenbelt Lake & $\mathrm{AC}$ & 350000100534701 & $07-13-99$ & $<.003$ & $<.007$ & $<.004$ & $<.013$ & $<.035$ \\
\hline Hubbard Creek Reservoir & P5 & 324913098581801 & $07-15-99$ & $<.003$ & $<.007$ & $<.004$ & $<.013$ & $<.035$ \\
\hline La Feria Reservoir & PS & 260822097493401 & $07-14-99$ & $<.003$ & $<.007$ & $<.004$ & $<.013$ & $<.035$ \\
\hline Lake Anahuac & PS & 294624094411201 & $07-21-99$ & $<.003$ & $<.007$ & $<.004$ & $<.013$ & $<.035$ \\
\hline Lake Belton & $\mathrm{BC}$ & 310711097302201 & 06-30-99 & $<.003$ & $<.007$ & $<.004$ & $<.013$ & $<.035$ \\
\hline Lake Bonham & PS & 333858096083301 & $07-27-99$ & $<.003$ & $<.007$ & $<.004$ & $<.013$ & $<.035$ \\
\hline Lake Bridgeport & Mid-lake & 331312097501801 & $07-21-99$ & $<.003$ & $<.007$ & $<.004$ & $<.013$ & $<.035$ \\
\hline Lake Brownwood & PS & 315026099005301 & 07-19-99 & $<.003$ & $<.007$ & $<.004$ & -- & $<.035$ \\
\hline Lake Houston & $\mathrm{BC}$ & 295702095091401 & $07-12-99$ & $<.003$ & $<.007$ & $<.004$ & $<.013$ & $<.035$ \\
\hline Lake J.B. Thomas & PS & 323510101085501 & $07-13-99$ & $<.003$ & $<.007$ & $<.004$ & $<.03$ & $<.035$ \\
\hline Lake Kickapoo & PS & 333913098480601 & 07-19-99 & $<.003$ & $<.007$ & $<.004$ & $<.013$ & $<.035$ \\
\hline Lake Limestone & $\mathrm{BC}$ & 312458096205101 & 07-08-99 & $<.003$ & $<.007$ & $<.004$ & -- & $<.035$ \\
\hline Lake Meredith & $\mathrm{BC}$ & 354113101360101 & 07-13-99 & $<.003$ & $<.007$ & $<.004$ & $<.013$ & $<.035$ \\
\hline Lake Mexia & PS & 313848096345001 & 07-07-99 & $<.003$ & $<.007$ & $<.004$ & $<.013$ & $<.035$ \\
\hline
\end{tabular}


Table 2. Concentrations of soluble pesticides in public water-supply reservoirs sampled in Texas, June-July 1999—Continued

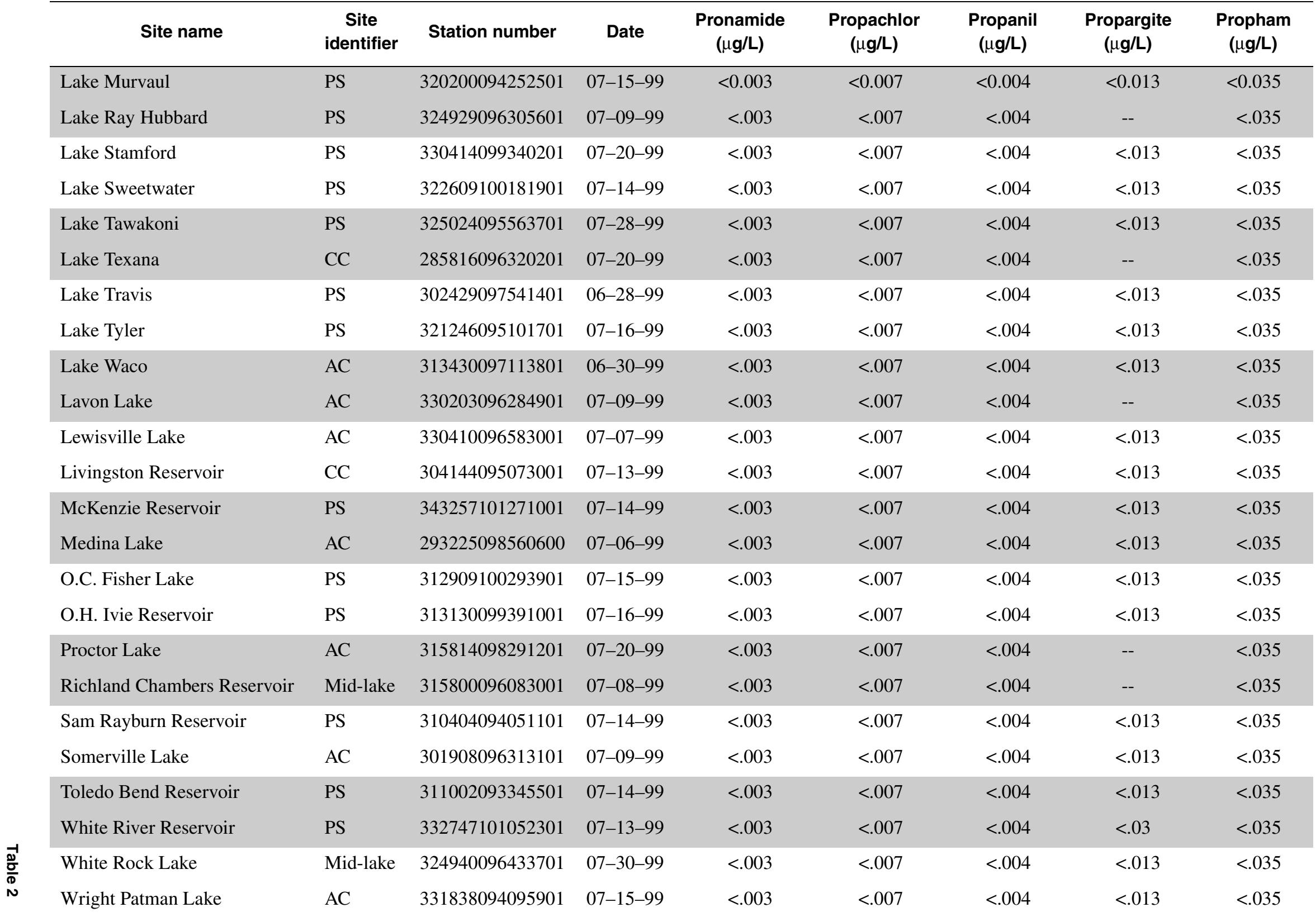


Table 2. Concentrations of soluble pesticides in public water-supply reservoirs sampled in Texas, June-July 1999-Continued

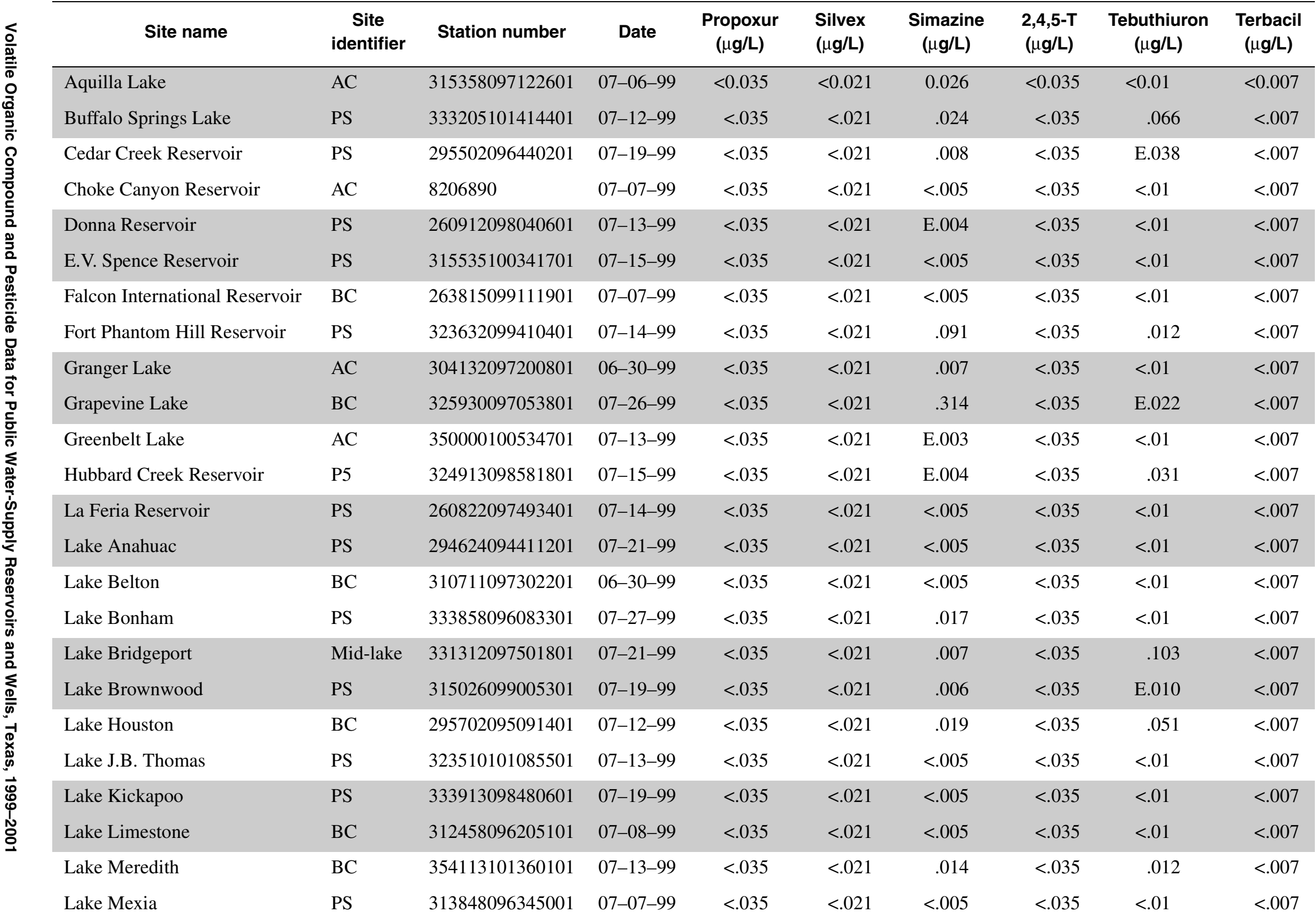


Table 2. Concentrations of soluble pesticides in public water-supply reservoirs sampled in Texas, June-July 1999—Continued

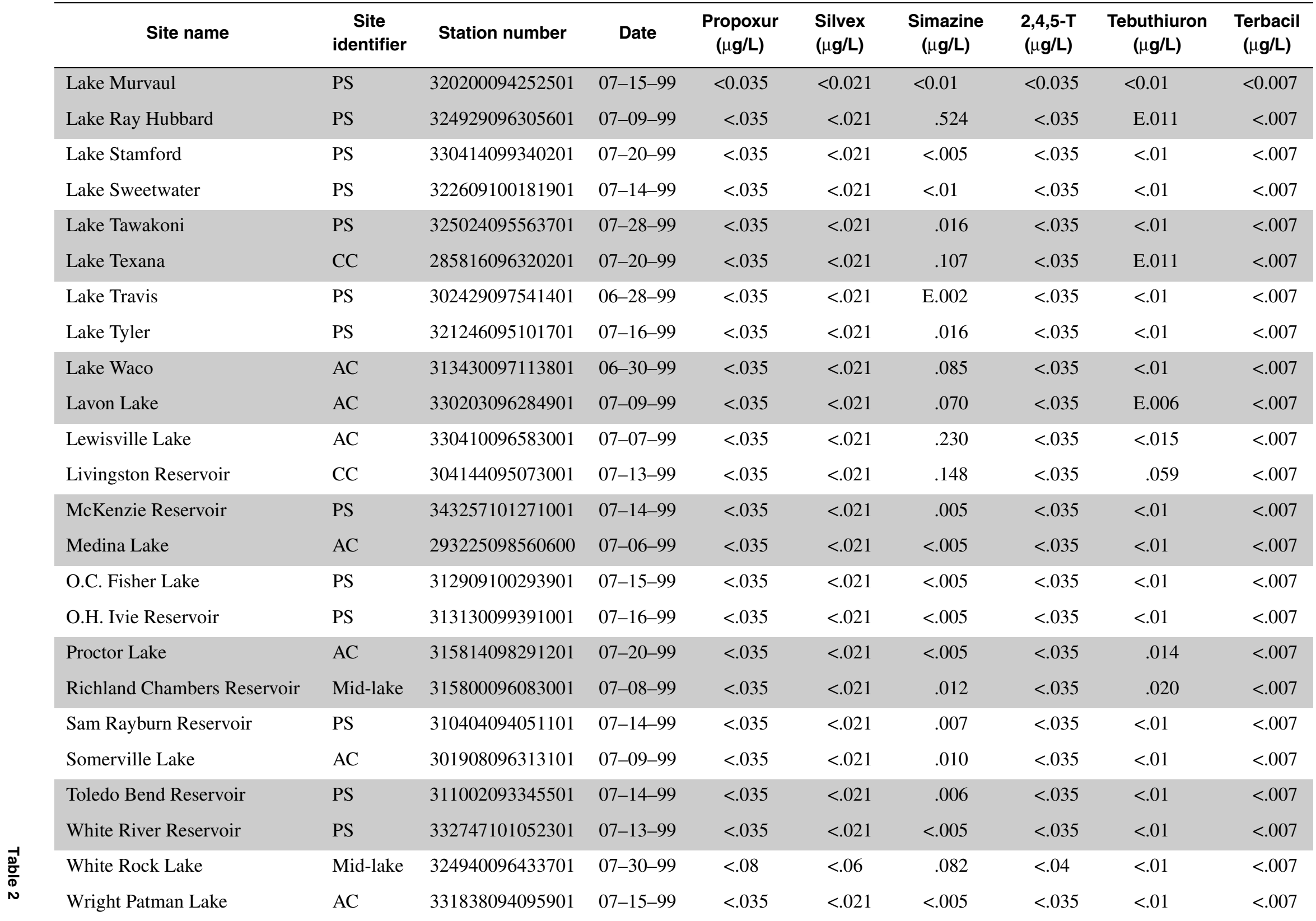


Table 2. Concentrations of soluble pesticides in public water-supply reservoirs sampled in Texas, June-July 1999-Continued

\begin{tabular}{|c|c|c|c|c|c|c|c|c|}
\hline Site name & $\begin{array}{c}\text { Site } \\
\text { identifier }\end{array}$ & Station number & Date & $\begin{array}{c}\text { Terbufos } \\
(\mu \mathrm{g} / \mathrm{L})\end{array}$ & $\begin{array}{c}\text { Thiobencarb } \\
(\mu \mathrm{g} / \mathrm{L})\end{array}$ & $\begin{array}{c}\text { Triallate } \\
(\mu \mathrm{g} / \mathrm{L})\end{array}$ & $\begin{array}{c}\text { Triclopyr } \\
(\mu \mathrm{g} / \mathrm{L})\end{array}$ & $\begin{array}{c}\text { Trifluralin } \\
(\mu \mathrm{g} / \mathrm{L})\end{array}$ \\
\hline Aquilla Lake & $\mathrm{AC}$ & 315358097122601 & $07-06-99$ & $<0.013$ & $<0.002$ & $<0.001$ & $<0.25$ & $<0.002$ \\
\hline Buffalo Springs Lake & PS & 333205101414401 & $07-12-99$ & $<.013$ & $<.002$ & $<.001$ & $<.25$ & $<.002$ \\
\hline Cedar Creek Reservoir & PS & 295502096440201 & 07-19-99 & $<.013$ & $<.002$ & $<.001$ & $<.25$ & $<.002$ \\
\hline Choke Canyon Reservoir & $\mathrm{AC}$ & 8206890 & 07-07-99 & $<.013$ & $<.002$ & $<.001$ & $<.25$ & $<.002$ \\
\hline Donna Reservoir & PS & 260912098040601 & $07-13-99$ & $<.013$ & $<.002$ & $<.001$ & $<.25$ & $<.002$ \\
\hline E.V. Spence Reservoir & PS & 315535100341701 & $07-15-99$ & $<.013$ & $<.002$ & $<.001$ & $<.25$ & $<.002$ \\
\hline Falcon International Reservoir & $\mathrm{BC}$ & 263815099111901 & 07-07-99 & $<.013$ & $<.002$ & $<.001$ & $<.25$ & $<.002$ \\
\hline Fort Phantom Hill Reservoir & PS & 323632099410401 & 07-14-99 & $<.013$ & $<.002$ & $<.001$ & $<.25$ & $<.002$ \\
\hline Granger Lake & $\mathrm{AC}$ & 304132097200801 & 06-30-99 & $<.013$ & $<.002$ & $<.001$ & $<.25$ & $<.002$ \\
\hline Grapevine Lake & $\mathrm{BC}$ & 325930097053801 & $07-26-99$ & $<.013$ & $<.002$ & $<.001$ & $<.25$ & $<.002$ \\
\hline Greenbelt Lake & $\mathrm{AC}$ & 350000100534701 & $07-13-99$ & $<.013$ & $<.002$ & $<.001$ & $<.25$ & $<.002$ \\
\hline Hubbard Creek Reservoir & P5 & 324913098581801 & $07-15-99$ & $<.013$ & $<.002$ & $<.001$ & $<.25$ & $<.002$ \\
\hline La Feria Reservoir & PS & 260822097493401 & $07-14-99$ & $<.013$ & $<.002$ & $<.001$ & $<.25$ & $<.002$ \\
\hline Lake Anahuac & PS & 294624094411201 & $07-21-99$ & $<.013$ & $<.002$ & $<.001$ & $<.25$ & $<.002$ \\
\hline Lake Belton & $\mathrm{BC}$ & 310711097302201 & 06-30-99 & $<.013$ & $<.002$ & $<.001$ & $<.25$ & $<.002$ \\
\hline Lake Bonham & PS & 333858096083301 & $07-27-99$ & $<.013$ & $<.002$ & $<.001$ & $<.25$ & $<.002$ \\
\hline Lake Bridgeport & Mid-lake & 331312097501801 & $07-21-99$ & $<.013$ & $<.002$ & $<.001$ & $<.25$ & $<.002$ \\
\hline Lake Brownwood & PS & 315026099005301 & 07-19-99 & $<.013$ & $<.002$ & $<.001$ & $<.25$ & $<.002$ \\
\hline Lake Houston & $\mathrm{BC}$ & 295702095091401 & $07-12-99$ & $<.013$ & $<.002$ & $<.001$ & $<.25$ & $<.002$ \\
\hline Lake J.B. Thomas & PS & 323510101085501 & $07-13-99$ & $<.013$ & $<.002$ & $<.001$ & $<.25$ & $<.002$ \\
\hline Lake Kickapoo & PS & 333913098480601 & 07-19-99 & $<.013$ & $<.002$ & $<.001$ & $<.25$ & $<.002$ \\
\hline Lake Limestone & $\mathrm{BC}$ & 312458096205101 & 07-08-99 & $<.013$ & $<.002$ & $<.001$ & $<.25$ & $<.002$ \\
\hline Lake Meredith & $\mathrm{BC}$ & 354113101360101 & $07-13-99$ & $<.013$ & $<.002$ & $<.001$ & $<.25$ & $<.002$ \\
\hline Lake Mexia & PS & 313848096345001 & 07-07-99 & $<.013$ & $<.002$ & $<.001$ & $<.25$ & $<.002$ \\
\hline
\end{tabular}


Table 2. Concentrations of soluble pesticides in public water-supply reservoirs sampled in Texas, June-July 1999-Continued

\begin{tabular}{|c|c|c|c|c|c|c|c|c|}
\hline Site name & $\begin{array}{c}\text { Site } \\
\text { identifier }\end{array}$ & Station number & Date & $\begin{array}{c}\text { Terbufos } \\
(\mu \mathrm{g} / \mathrm{L})\end{array}$ & $\begin{array}{c}\text { Thiobencarb } \\
(\mu \mathrm{g} / L)\end{array}$ & $\begin{array}{c}\text { Triallate } \\
(\mu \mathrm{g} / \mathrm{L})\end{array}$ & $\begin{array}{l}\text { Triclopyr } \\
(\mu \mathrm{g} / \mathrm{L})\end{array}$ & $\begin{array}{c}\text { Trifluralin } \\
(\mu \mathrm{g} / \mathrm{L})\end{array}$ \\
\hline Lake Murvaul & PS & 320200094252501 & $07-15-99$ & $<0.013$ & $<0.002$ & $<0.001$ & $<0.25$ & $<0.002$ \\
\hline Lake Ray Hubbard & PS & 324929096305601 & 07-09-99 & $<.013$ & $<.002$ & $<.001$ & $<.25$ & $<.002$ \\
\hline Lake Stamford & PS & 330414099340201 & 07-20-99 & $<.013$ & $<.002$ & $<.001$ & $<.25$ & $<.002$ \\
\hline Lake Sweetwater & PS & 322609100181901 & 07-14-99 & $<.013$ & $<.002$ & $<.001$ & $<.25$ & $<.002$ \\
\hline Lake Tawakoni & PS & 325024095563701 & $07-28-99$ & $<.013$ & $<.002$ & $<.001$ & $<.25$ & $<.002$ \\
\hline Lake Texana & $\mathrm{CC}$ & 285816096320201 & 07-20-99 & $<.013$ & $<.002$ & $<.001$ & $<.25$ & $<.002$ \\
\hline Lake Travis & PS & 302429097541401 & 06-28-99 & $<.013$ & $<.002$ & $<.001$ & $<.25$ & $<.002$ \\
\hline Lake Tyler & PS & 321246095101701 & 07-16-99 & $<.013$ & $<.002$ & $<.001$ & $<.25$ & $<.002$ \\
\hline Lake Waco & $\mathrm{AC}$ & 313430097113801 & 06-30-99 & $<.013$ & $<.002$ & $<.001$ & $<.25$ & $<.002$ \\
\hline Lavon Lake & $\mathrm{AC}$ & 330203096284901 & 07-09-99 & $<.013$ & $<.002$ & $<.001$ & $<.25$ & $<.002$ \\
\hline Lewisville Lake & $\mathrm{AC}$ & 330410096583001 & 07-07-99 & $<.013$ & $<.002$ & $<.001$ & $<.25$ & $<.002$ \\
\hline Livingston Reservoir & $\mathrm{CC}$ & 304144095073001 & 07-13-99 & $<.013$ & $<.002$ & $<.001$ & $<.25$ & $<.002$ \\
\hline McKenzie Reservoir & PS & 343257101271001 & 07-14-99 & $<.013$ & $<.002$ & $<.001$ & $<.25$ & $<.002$ \\
\hline Medina Lake & $\mathrm{AC}$ & 293225098560600 & 07-06-99 & $<.013$ & $<.002$ & $<.001$ & $<.25$ & $<.002$ \\
\hline O.C. Fisher Lake & PS & 312909100293901 & $07-15-99$ & $<.013$ & $<.002$ & $<.001$ & $<.25$ & $<.002$ \\
\hline O.H. Ivie Reservoir & PS & 313130099391001 & $07-16-99$ & $<.013$ & $<.002$ & $<.001$ & $<.25$ & $<.002$ \\
\hline Proctor Lake & $\mathrm{AC}$ & 315814098291201 & 07-20-99 & $<.013$ & $<.002$ & $<.001$ & $<.25$ & $<.002$ \\
\hline Richland Chambers Reservoir & Mid-lake & 315800096083001 & 07-08-99 & $<.013$ & $<.002$ & $<.001$ & $<.25$ & $<.002$ \\
\hline Sam Rayburn Reservoir & PS & 310404094051101 & 07-14-99 & $<.013$ & $<.002$ & $<.001$ & $<.25$ & $<.002$ \\
\hline Somerville Lake & $\mathrm{AC}$ & 301908096313101 & 07-09-99 & $<.013$ & $<.002$ & $<.001$ & $<.25$ & $<.002$ \\
\hline Toledo Bend Reservoir & PS & 311002093345501 & 07-14-99 & $<.013$ & $<.002$ & $<.001$ & $<.25$ & $<.002$ \\
\hline White River Reservoir & PS & 332747101052301 & 07-13-99 & $<.013$ & $<.002$ & $<.001$ & $<.25$ & $<.002$ \\
\hline White Rock Lake & Mid-lake & 324940096433701 & 07-30-99 & $<.013$ & $<.002$ & $<.001$ & $<.25$ & $<.002$ \\
\hline Wright Patman Lake & $\mathrm{AC}$ & 331838094095901 & 07-15-99 & $<.013$ & $<.002$ & $<.001$ & $<.25$ & $<.002$ \\
\hline
\end{tabular}


\& Table 3. Summary of soluble pesticides in samples collected from public water-supply reservoirs in Texas, June-July 1999 (only pesticides with one or more detections are shown)

$\underset{\delta}{\varrho}[\mu \mathrm{g} / \mathrm{L}$, micrograms per liter; $\mathrm{MCL}$, maximum contaminant level; --, not applicable]

\begin{tabular}{|c|c|c|c|c|}
\hline Pesticide & $\begin{array}{l}\text { Method detection limit } \\
\qquad(\mu \mathrm{g} / \mathrm{L})\end{array}$ & $\begin{array}{l}\text { Detection frequency } \\
\text { (percent) }\end{array}$ & $\begin{array}{c}\text { Maximum concentration } \\
(\mu \mathbf{g} / L)\end{array}$ & $\begin{array}{c}M C L \\
(\mu g / L)\end{array}$ \\
\hline Atrazine & 0.001 & 96 & 1.40 & 3.0 \\
\hline Deethylatrazine & .002 & 90 & .311 & -- \\
\hline Simazine & .005 & 60 & .524 & 4.0 \\
\hline Metolachlor & .002 & 58 & .287 & -- \\
\hline Prometon & .018 & 40 & .142 & -- \\
\hline Diuron & .06 & 35 & .76 & -- \\
\hline Tebuthiuron & .01 & 31 & .103 & -- \\
\hline Diazinon & .002 & 25 & .065 & -- \\
\hline Alachlor & .002 & 10 & .286 & 2.0 \\
\hline Azinphos-methyl & .001 & 6.3 & .033 & -- \\
\hline Chlorpyrifos & .002 & 6.3 & .004 & -- \\
\hline Fluometuron & .06 & 6.3 & .25 & -- \\
\hline Malathion & .005 & 6.3 & .057 & -- \\
\hline 2,4-D & .11 & 4.2 & .25 & 70 \\
\hline Parathion-methyl & .006 & 4.2 & .034 & -- \\
\hline Dieldrin & .001 & 2.1 & .007 & -- \\
\hline Fenuron & .013 & 2.1 & .08 & -- \\
\hline Metribuzin & .004 & 2.1 & .017 & -- \\
\hline Molinate & .004 & 2.1 & .24 & -- \\
\hline
\end{tabular}


Table 4. Concentrations of volatile organic compounds, nitrite plus nitrate, and tritium in public water-supply wells sampled in Texas, November 1999-January 2001

[ $\mu \mathrm{g} / \mathrm{L}$, micrograms per liter; mg/L, milligrams per liter; $\mathrm{pCi} / \mathrm{L}$, picocuries per liter; <, less than; --, not analyzed; E, estimated]

\begin{tabular}{|c|c|c|c|c|c|c|c|c|c|c|c|}
\hline $\begin{array}{c}\text { Local } \\
\text { identifier }\end{array}$ & $\begin{array}{l}\text { Station } \\
\text { number }\end{array}$ & Date & $\begin{array}{l}\text { MTBE } \\
(\mu \mathrm{g} / \mathrm{L})\end{array}$ & $\begin{array}{c}\text { Benzene } \\
(\mu \mathrm{g} / \mathrm{L})\end{array}$ & $\begin{array}{c}\text { Toluene } \\
(\mu \mathrm{g} / \mathrm{L})\end{array}$ & $\begin{array}{c}\text { Ethyl- } \\
\text { benzene } \\
(\mu \mathrm{g} / \mathrm{L})\end{array}$ & $\begin{array}{c}\text { Total } \\
\text { xylene } \\
(\mu \mathrm{g} / \mathrm{L})\end{array}$ & $\begin{array}{c}\text { o-Xylene } \\
(\mu \mathrm{g} / \mathrm{L})\end{array}$ & $\begin{array}{c}m \text { - and } p- \\
\text { Xylene } \\
(\mu \mathrm{g} / \mathrm{L})\end{array}$ & $\begin{array}{c}\mathrm{NO}_{2}+ \\
\mathrm{NO}_{3} \\
(\mathrm{mg} / \mathrm{L})\end{array}$ & $\begin{array}{l}\text { Tritium } \\
\text { (pCi/L) }\end{array}$ \\
\hline AB-27-37-103 & 322805102283801 & $05-17-00$ & $<0.2$ & $<0.2$ & $<0.2$ & $<0.2$ & $<0.2$ & $<0.2$ & $<0.2$ & 1.82 & $<2.5$ \\
\hline AK-06-52-506 & 351024101334401 & 05-04-00 & $<.2$ & $<.2$ & $<.2$ & $<.2$ & $<.2$ & $<.2$ & $<.2$ & 1.34 & $<2.5$ \\
\hline AP-59-62-403 & 300259096195001 & 04-03-00 & $<.2$ & $<.2$ & $<.2$ & $<.2$ & $<.2$ & $<.2$ & $<.2$ & .263 & $<2.5$ \\
\hline AP-66-16-407 & 294903096061401 & $04-21-00$ & $<.2$ & $<.2$ & $<.2$ & $<.2$ & $<.2$ & $<.2$ & $<.2$ & .603 & $<2.5$ \\
\hline AT-58-62-206 & 300646097193001 & 12-10-99 & $<.2$ & $<.2$ & $<.2$ & $<.2$ & $<.2$ & $<.2$ & $<.2$ & .411 & 14.7 \\
\hline AU-21-30-389 & 333612099163101 & $05-02-00$ & $<.2$ & $<.2$ & $<.2$ & $<.2$ & $<.2$ & $<.2$ & $<.2$ & 10.2 & 20.8 \\
\hline AX-58-04-604 & 305718097314401 & $03-22-00$ & $<.2$ & $<.2$ & $<.2$ & $<.2$ & $<.2$ & $<.2$ & $<.2$ & 7.76 & 9.6 \\
\hline AX-58-04-623 & 305624097321101 & $03-22-00$ & $<.2$ & $<.2$ & $<.2$ & $<.2$ & $<.2$ & $<.2$ & $<.2$ & $<.05$ & 5.8 \\
\hline AY-68-35-105 & 292842098425001 & $03-06-00$ & $<.2$ & $<.2$ & $<.2$ & $<.2$ & $<.2$ & $<.2$ & $<.2$ & 1.04 & 9.9 \\
\hline BK-73-52-905 & 290819103312601 & $12-28-00$ & $<.2$ & $<.2$ & $<.2$ & $<.2$ & $<.2$ & $<.2$ & $<.2$ & $<.047$ & 16.6 \\
\hline BL-11-38-334 & 342841101155301 & $05-01-00$ & $<.2$ & $<.2$ & $<.2$ & $<.2$ & $<.2$ & $<.2$ & $<.2$ & .580 & $<2.5$ \\
\hline BL-11-48-507 & 341919101035201 & $05-02-00$ & $<.2$ & $<.2$ & $<.2$ & $<.2$ & $<.2$ & $<.2$ & $<.2$ & 14.6 & 25.9 \\
\hline BL-12-41-204 & 342109100570301 & 05-02-00 & $<.2$ & $<.2$ & $<.2$ & $<.2$ & $<.2$ & $<.2$ & $<.2$ & 14.9 & 20.2 \\
\hline BR-41-01-244 & 315849098552101 & $04-05-00$ & $<.2$ & $<.2$ & $<.2$ & $<.2$ & $<.2$ & $<.2$ & $<.2$ & 1.31 & 13.1 \\
\hline BT-57-14-403 & 304939098202401 & 03-08-00 & $<.2$ & $<.2$ & $<.2$ & $<.2$ & $<.2$ & $<.2$ & $<.2$ & .062 & $<2.5$ \\
\hline BT-57-14-404 & 304927098211201 & $03-08-00$ & $<.2$ & $<.2$ & $<.2$ & $<.2$ & $<.2$ & $<.2$ & $<.2$ & $<.05$ & 2.9 \\
\hline BT-57-21-607 & 304125098224001 & $03-28-00$ & $<.2$ & $<.2$ & $<.2$ & $<.2$ & $<.2$ & $<.2$ & $<.2$ & .456 & 10.9 \\
\hline BT-57-21-608 & 304121098224201 & $03-28-00$ & $<.2$ & $<.2$ & $<.2$ & $<.2$ & $<.2$ & $<.2$ & $<.2$ & .388 & 7.7 \\
\hline BT-57-23-110 & 304406098130801 & 03-29-00 & $<.2$ & $<.2$ & $<.2$ & $<.2$ & $<.2$ & $<.2$ & $<.2$ & 5.37 & 12.2 \\
\hline BT-57-30-101 & 303548098211701 & $03-01-00$ & .49 & $<.2$ & $<.2$ & $<.2$ & $<.2$ & $<.2$ & $<.2$ & 5.18 & 14.1 \\
\hline
\end{tabular}


Table 4. Concentrations of volatile organic compounds, nitrite plus nitrate, and tritium in public water-supply wells sampled in Texas, November 1999-January 2001-Continued

\begin{tabular}{|c|c|c|c|c|c|c|c|c|c|c|c|}
\hline $\begin{array}{c}\text { Local } \\
\text { identifier }\end{array}$ & $\begin{array}{l}\text { Station } \\
\text { number }\end{array}$ & Date & $\begin{array}{l}\text { MTBE } \\
(\mu \mathrm{g} / \mathrm{L})\end{array}$ & $\begin{array}{c}\text { Benzene } \\
(\mu \mathrm{g} / \mathrm{L})\end{array}$ & $\begin{array}{c}\text { Toluene } \\
\text { ( } \mu \mathrm{g} / \mathrm{L})\end{array}$ & $\begin{array}{c}\text { Ethyl- } \\
\text { benzene } \\
(\mu \mathrm{g} / \mathrm{L})\end{array}$ & $\begin{array}{c}\text { Total } \\
\text { xylene } \\
(\mu \mathrm{g} / \mathrm{L})\end{array}$ & $\begin{array}{c}\text { o-Xylene } \\
\text { ( } \mu \mathrm{g} / \mathrm{L})\end{array}$ & $\begin{array}{c}m \text { - and } p \text { - } \\
\text { Xylene } \\
(\mu \mathrm{g} / \mathrm{L})\end{array}$ & $\begin{array}{c}\mathrm{NO}_{2}+ \\
\mathrm{NO}_{3} \\
(\mathrm{mg} / \mathrm{L})\end{array}$ & $\begin{array}{l}\text { Tritium } \\
\text { (pCi/L) }\end{array}$ \\
\hline $\mathrm{BX}-30-55-936$ & 320939099093801 & $12-18-00$ & $<0.2$ & $<0.2$ & $<0.2$ & $<0.2$ & $<0.2$ & $<0.2$ & $<0.2$ & 6.25 & 15.7 \\
\hline DA-06-28-202 & 353704101343701 & $05-03-00$ & $<.2$ & $<.2$ & $<.2$ & $<.2$ & $<.2$ & $<.2$ & $<.2$ & 1.99 & 3.2 \\
\hline DL-14-62-604 & 340406098160701 & $05-10-00$ & $<.2$ & $<.2$ & .11 & $<.2$ & $<.2$ & $<.2$ & $<.2$ & 3.48 & 20.5 \\
\hline DP-24-18-308 & 334352102450601 & 05-09-00 & $<.2$ & $<.2$ & $<.2$ & $<.2$ & $<.2$ & $<.2$ & $<.2$ & 9.97 & 4.2 \\
\hline DP-25-24-304 & 334245103011001 & $05-08-00$ & $<.2$ & $<.2$ & $<.2$ & $<.2$ & $<.2$ & $<.2$ & $<.2$ & 1.15 & $<2.5$ \\
\hline DU-12-06-609 & 345658100155501 & $05-08-00$ & $<.2$ & $<.2$ & $<.2$ & $<.2$ & $<.2$ & $<.2$ & $<.2$ & 8.74 & 25.9 \\
\hline DX-68-05-621 & 295520098242301 & $03-22-00$ & $<.2$ & $<.2$ & $<.2$ & $<.2$ & $<.2$ & $<.2$ & $<.2$ & 1.02 & $<2.5$ \\
\hline DX-68-06-405 & 295528098222301 & 03-22-00 & $<.2$ & $<.2$ & $<.2$ & $<.2$ & $<.2$ & $<.2$ & $<.2$ & .980 & 3.8 \\
\hline DY-41-13-307 & 315105098241401 & $04-05-00$ & $<.2$ & $<.2$ & $<.2$ & $<.2$ & $<.2$ & $<.2$ & $<.2$ & 7.06 & 6.4 \\
\hline $\mathrm{HH}-45-35-505$ & 312715102402201 & $01-12-00$ & $<.2$ & $<.2$ & $<.2$ & $<.2$ & $<.2$ & $<.2$ & $<.2$ & 1.49 & -- \\
\hline HP-12-62-802 & 340210100184901 & $05-05-00$ & $<.2$ & $<.2$ & .12 & $<.2$ & $<.2$ & $<.2$ & $<.2$ & 9.37 & 15.0 \\
\hline HS-28-09-404 & 324810101591201 & $05-16-00$ & -- & -- & -- & -- & -- & -- & -- & 6.50 & 4.5 \\
\hline HT-07-63-204 & 350607102103401 & $05-03-00$ & $<.2$ & $<.2$ & $<.2$ & $<.2$ & $<.2$ & $<.2$ & $<.2$ & .707 & $<2.5$ \\
\hline HZ-77-34-606 & 282608099455301 & $03-28-00$ & $<.2$ & $<.2$ & $<.2$ & $<.2$ & $<.2$ & $<.2$ & $<.2$ & $<.05$ & $<2.5$ \\
\hline JA-05-57-613 & 350254100535501 & $05-04-00$ & $<.2$ & $<.2$ & .27 & $<.2$ & $<.2$ & $<.2$ & $<.2$ & 7.11 & 14.7 \\
\hline JA-12-12-404 & 344910100360401 & $05-02-00$ & $<.2$ & $<.2$ & $<.2$ & $<.2$ & $<.2$ & $<.2$ & $<.2$ & 4.60 & 12.5 \\
\hline JH-45-05-924 & 315245102240201 & $05-25-00$ & $<.2$ & .11 & .11 & $<.2$ & $<.2$ & $<.2$ & $<.2$ & 7.19 & 16.3 \\
\hline JL-49-04-116 & 315757106370201 & $01-06-00$ & $<.2$ & $<.2$ & $<.2$ & $<.2$ & $<.2$ & $<.2$ & $<.2$ & $<.05$ & 17.3 \\
\hline JL-49-04-423 & 315708106362301 & 01-03-00 & $<.2$ & $<.2$ & $<.2$ & $<.2$ & $<.2$ & $<.2$ & $<.2$ & $<.05$ & 8.0 \\
\hline JL-49-04-428 & 315517106361401 & 01-06-00 & $<.2$ & $<.2$ & $<.2$ & $<.2$ & $<.2$ & $<.2$ & $<.2$ & $<.05$ & 39.7 \\
\hline JL-49-04-492 & 315623106360601 & $01-06-00$ & $<.2$ & $<.2$ & $<.2$ & $<.2$ & $<.2$ & $<.2$ & $<.2$ & $<.05$ & 45.4 \\
\hline JL-49-22-626 & 314145106163601 & $01-04-00$ & $<.2$ & $<.2$ & $<.2$ & $<.2$ & $<.2$ & $<.2$ & $<.2$ & .184 & $<2.5$ \\
\hline JL-49-24-423 & 314107106063301 & 01-07-00 & $<.2$ & $<.2$ & $<.2$ & $<.2$ & $<.2$ & $<.2$ & $<.2$ & 11.4 & 12.8 \\
\hline JL-49-40-502 & 312648106044701 & 01-04-00 & $<.2$ & $<.2$ & $<.2$ & $<.2$ & $<.2$ & $<.2$ & $<.2$ & $<.05$ & $<2.5$ \\
\hline$J Y-65-26-520$ & 293314095474702 & 04-03-00 & $<.2$ & $<.2$ & $<.2$ & $<.2$ & $<.2$ & $<.2$ & $<.2$ & $<.05$ & $<2.5$ \\
\hline KD-27-01-612 & 325523102542701 & $05-22-00$ & $<.2$ & $<.2$ & $<.2$ & $<.2$ & $<.2$ & $<.2$ & $<.2$ & 2.66 & $<2.5$ \\
\hline KK-57-42-709 & 301545098502801 & $07-06-00$ & $<.2$ & $<.2$ & $<.2$ & $<.2$ & $<.2$ & $<.2$ & $<.2$ & .459 & $<2.5$ \\
\hline $\mathrm{KK}-57-50-325$ & 301357098471901 & 03-28-00 & $<.2$ & $<.2$ & $<.2$ & $<.2$ & $<.2$ & $<.2$ & $<.2$ & 2.40 & 3.8 \\
\hline $\mathrm{KK}-57-51-303$ & 301411098383101 & $03-21-00$ & $<.2$ & $<.2$ & $<.2$ & $<.2$ & $<.2$ & $<.2$ & $<.2$ & 2.63 & 10.2 \\
\hline KK-57-52-107 & 301430098363401 & $03-21-00$ & $<.2$ & $<.2$ & $<.2$ & $<.2$ & $<.2$ & $<.2$ & $<.2$ & 1.31 & 4.8 \\
\hline
\end{tabular}


Table 4. Concentrations of volatile organic compounds, nitrite plus nitrate, and tritium in public water-supply wells sampled in Texas, November 1999-January 2001-Continued

\begin{tabular}{|c|c|c|c|c|c|c|c|c|c|c|c|}
\hline $\begin{array}{c}\text { Local } \\
\text { identifier }\end{array}$ & $\begin{array}{l}\text { Station } \\
\text { number }\end{array}$ & Date & $\begin{array}{l}\text { MTBE } \\
(\mu \mathrm{g} / \mathrm{L})\end{array}$ & $\begin{array}{c}\text { Benzene } \\
(\mu \mathrm{g} / \mathrm{L})\end{array}$ & $\begin{array}{c}\text { Toluene } \\
(\mu \mathrm{g} / \mathrm{L})\end{array}$ & $\begin{array}{c}\text { Ethyl- } \\
\text { benzene } \\
(\mu \mathrm{g} / \mathrm{L})\end{array}$ & $\begin{array}{c}\text { Total } \\
\text { xylene } \\
(\mu \mathrm{g} / \mathrm{L})\end{array}$ & $\begin{array}{c}\text { o-Xylene } \\
(\mu \mathrm{g} / \mathrm{L})\end{array}$ & $\begin{array}{c}m \text { - and } p \text { - } \\
\text { Xylene } \\
(\mu \mathrm{g} / \mathrm{L})\end{array}$ & $\begin{array}{c}\mathrm{NO}_{2}+ \\
\mathrm{NO}_{3} \\
(\mathrm{mg} / \mathrm{L})\end{array}$ & $\begin{array}{l}\text { Tritium } \\
\text { (pCi/L) }\end{array}$ \\
\hline KP-79-23-101 & 284256097125001 & $02-29-00$ & $<0.2$ & $<0.2$ & $<0.2$ & $<0.2$ & $<0.2$ & $<0.2$ & $<0.2$ & $<0.05$ & $<2.5$ \\
\hline KT-18-11-405 & 334844096435701 & $04-19-00$ & $<.2$ & $<.2$ & $<.2$ & $<.2$ & $<.2$ & $<.2$ & $<.2$ & 1.19 & 17.9 \\
\hline KT-18-11-406 & 334840096434001 & 04-19-00 & $<.2$ & $<.2$ & $<.2$ & $<.2$ & $<.2$ & $<.2$ & $<.2$ & 2.10 & 16.0 \\
\hline KT-18-28-703 & 333039096362901 & 04-19-00 & $<.2$ & $<.2$ & $<.2$ & $<.2$ & $<.2$ & $<.2$ & $<.2$ & $<.05$ & $<2.5$ \\
\hline KY-11-57-602 & 340345101531801 & $05-03-00$ & $<.2$ & $<.2$ & $<.2$ & $<.2$ & $<.2$ & $<.2$ & $<.2$ & 8.09 & 35.5 \\
\hline KZ-12-19-104 & 344234100431601 & 05-09-00 & $<.2$ & $<.2$ & .10 & $<.2$ & $<.2$ & $<.2$ & $<.2$ & 16.0 & 20.5 \\
\hline LD-13-44-907 & 341523099305701 & $12-19-00$ & $<.2$ & $<.2$ & $<.2$ & $<.2$ & $<.2$ & $<.2$ & $<.2$ & 11.3 & 20.2 \\
\hline LJ-60-64-807 & 300149095045001 & $05-04-00$ & $<.2$ & $<.2$ & $<.2$ & $<.2$ & $<.2$ & $<.2$ & $<.2$ & $<.05$ & $<2.5$ \\
\hline LP-21-49-317 & 331233099545201 & $05-01-00$ & $<.2$ & $<.2$ & $<.2$ & $<.2$ & $<.2$ & $<.2$ & $<.2$ & 11.2 & 37.4 \\
\hline LX-24-40-405 & 332624102072901 & $05-17-00$ & $<.2$ & $<.2$ & $<.2$ & $<.2$ & $<.2$ & $<.2$ & $<.2$ & 6.78 & $<2.5$ \\
\hline LY-32-26-706 & 323204097500301 & $03-22-00$ & $<.2$ & $<.2$ & $<.2$ & $<.2$ & $<.2$ & $<.2$ & $<.2$ & $<.05$ & 13.4 \\
\hline LY-32-34-208 & 322958097481301 & $03-21-00$ & $<.2$ & $<.2$ & $<.2$ & $<.2$ & $<.2$ & $<.2$ & $<.2$ & $<.05$ & 9.0 \\
\hline LY-32-34-303 & 322928097461701 & 04-03-00 & $<.2$ & $<.2$ & $<.2$ & $<.2$ & $<.2$ & $<.2$ & $<.2$ & $<.05$ & $<2.5$ \\
\hline LY-32-34-510 & 322537097485701 & $03-21-00$ & $<.2$ & $<.2$ & $<.2$ & $<.2$ & $<.2$ & $<.2$ & $<.2$ & $<.05$ & $<2.5$ \\
\hline LY-32-34-609 & 322626097454801 & $03-21-00$ & $<.2$ & $<.2$ & $<.2$ & $<.2$ & $<.2$ & $<.2$ & $<.2$ & .404 & 2.9 \\
\hline PB-28-62-105 & 320649101215501 & $04-26-00$ & $<.2$ & $<.2$ & $<.2$ & $<.2$ & $<.2$ & $<.2$ & $<.2$ & 3.31 & $<2.5$ \\
\hline PK-43-50-216 & 311422100490201 & $12-20-00$ & $<.2$ & $<.2$ & $<.2$ & $<.2$ & $<.2$ & $<.2$ & $<.2$ & 2.28 & 6.4 \\
\hline PS-52-02-404 & 305502103504101 & $01-10-00$ & $<.2$ & $<.2$ & $<.2$ & $<.2$ & $<.2$ & $<.2$ & $<.2$ & 1.10 & 17.6 \\
\hline PU-84-43-504 & 271841098402801 & 04-04-00 & $<.2$ & $<.2$ & $<.2$ & $<.2$ & $<.2$ & $<.2$ & $<.2$ & 3.17 & $<2.5$ \\
\hline PX-32-47-109 & 322201097141601 & $03-20-00$ & $<.2$ & $<.2$ & $<.2$ & $<.2$ & $<.2$ & $<.2$ & $<.2$ & $<.05$ & -- \\
\hline
\end{tabular}


Table 4. Concentrations of volatile organic compounds, nitrite plus nitrate, and tritium in public water-supply wells sampled in Texas, November 1999-January 2001-Continued

\begin{tabular}{|c|c|c|c|c|c|c|c|c|c|c|c|}
\hline $\begin{array}{c}\text { Local } \\
\text { identifier }\end{array}$ & $\begin{array}{l}\text { Station } \\
\text { number }\end{array}$ & Date & $\begin{array}{l}\text { MTBE } \\
(\mu \mathrm{g} / \mathrm{L})\end{array}$ & $\begin{array}{c}\text { Benzene } \\
(\mu \mathrm{g} / \mathrm{L})\end{array}$ & $\begin{array}{c}\text { Toluene } \\
\text { ( } \mu \mathrm{g} / \mathrm{L})\end{array}$ & $\begin{array}{c}\text { Ethyl- } \\
\text { benzene } \\
(\mu \mathrm{g} / \mathrm{L})\end{array}$ & $\begin{array}{c}\text { Total } \\
\text { xylene } \\
(\mu \mathrm{g} / \mathrm{L})\end{array}$ & $\begin{array}{c}\text { o-Xylene } \\
(\mu \mathrm{g} / \mathrm{L})\end{array}$ & $\begin{array}{c}m \text { - and } p- \\
\text { Xylene } \\
(\mu \mathrm{g} / \mathrm{L})\end{array}$ & $\begin{array}{c}\mathrm{NO}_{2}+ \\
\mathrm{NO}_{3} \\
(\mathrm{mg} / \mathrm{L})\end{array}$ & $\begin{array}{l}\text { Tritium } \\
\text { (pCi/L) }\end{array}$ \\
\hline RU-10-60-606 & 340427102302801 & $05-02-00$ & $<0.2$ & $0<.2$ & $<0.2$ & $<0.2$ & $<0.2$ & $<0.2$ & $<0.2$ & 7.14 & 7.7 \\
\hline $\mathrm{RW}-41-63-520$ & 310238098104001 & $03-23-00$ & $<.2$ & $<.2$ & 1.2 & $<.2$ & $<.2$ & $<.2$ & $<.2$ & $<.05$ & 2.6 \\
\hline SK-57-19-201 & 304323098421701 & 03-08-00 & $<.2$ & $<.2$ & $<.2$ & $<.2$ & $<.2$ & $<.2$ & $<.2$ & 2.55 & $<2.5$ \\
\hline SK-57-34-201 & 303001098485201 & 03-09-00 & $<.2$ & $<.2$ & $<.2$ & $<.2$ & $<.2$ & $<.2$ & $<.2$ & .120 & 6.7 \\
\hline SP-23-17-417 & 334043101585701 & $05-10-00$ & $<.2$ & $<.2$ & $<.2$ & $<.2$ & $<.2$ & $<.2$ & $<.2$ & 2.65 & 66.9 \\
\hline SP-23-18-107 & 334306101504101 & $05-10-00$ & $<.2$ & $<.2$ & $<.2$ & $<.2$ & $<.2$ & $<.2$ & $<.2$ & 11.9 & 8.6 \\
\hline SP-23-19-812 & 333955101404601 & $05-10-00$ & $<.2$ & $<.2$ & $<.2$ & $<.2$ & $<.2$ & $<.2$ & $<.2$ & 1.64 & $<2.5$ \\
\hline SP-24-24-214 & 334419102045201 & $05-18-00$ & $<.2$ & $<.2$ & $<.2$ & $<.2$ & $<.2$ & $<.2$ & $<.2$ & 3.96 & 62.1 \\
\hline SR-23-41-602 & 331933101543701 & $05-15-00$ & $<.2$ & .53 & $<.2$ & $<.2$ & $<.2$ & $<.2$ & $<.2$ & 7.52 & $<2.5$ \\
\hline SS-42-52-504 & 311202099321401 & $03-01-00$ & $<.2$ & $<.2$ & $<.2$ & $<.2$ & $<.2$ & $<.2$ & $<.2$ & $<.05$ & $<2.5$ \\
\hline ST-40-39-304 & 312745097083301 & $03-23-00$ & $<.2$ & $<.2$ & $<.2$ & $<.2$ & $<.2$ & $<.2$ & $<.2$ & $<.05$ & $<2.5$ \\
\hline SY-27-39-505 & 322555102105501 & $05-24-00$ & $<.2$ & $<.2$ & $<.2$ & $<.2$ & $<.2$ & $<.2$ & $<.2$ & 1.66 & $<2.5$ \\
\hline SY-28-42-803 & 321619101495001 & $05-23-00$ & $<.2$ & $<.2$ & $<.2$ & $<.2$ & $<.2$ & $<.2$ & $<.2$ & 7.12 & $<2.5$ \\
\hline SY-28-50-908 & 320816101474601 & $05-23-00$ & $<.2$ & $<.2$ & $<.2$ & $<.2$ & $<.2$ & $<.2$ & $<.2$ & 10.2 & 17.3 \\
\hline TB-76-03-605 & 285657100373801 & $03-28-00$ & $<.2$ & $<.2$ & $<.2$ & $<.2$ & $<.2$ & $<.2$ & $<.2$ & 1.40 & 8.6 \\
\hline TJ-27-63-712 & 320143102123101 & $12-20-00$ & $<.2$ & $<.2$ & $<.2$ & $<.2$ & $<.2$ & $<.2$ & $<.2$ & 5.33 & 3.8 \\
\hline TJ-28-57-702 & 320221101594901 & $05-25-00$ & $<.2$ & $<.2$ & $<.2$ & $<.2$ & $<.2$ & $<.2$ & $<.2$ & 8.91 & 3.8 \\
\hline TJ-28-57-903 & 320010101523701 & $05-23-00$ & $<.2$ & $<.2$ & $<.2$ & $<.2$ & $<.2$ & $<.2$ & $<.2$ & 3.63 & $<2.5$ \\
\hline TJ-45-07-407 & 315643102131101 & $05-24-00$ & $<.2$ & $<.2$ & $<.2$ & $<.2$ & $<.2$ & $<.2$ & $<.2$ & 3.81 & $<2.5$ \\
\hline TJ-45-07-606 & 315702102075401 & $05-25-00$ & $<.2$ & $<.2$ & $<.2$ & $<.2$ & $<.2$ & $<.2$ & $<.2$ & 1.91 & 15.4 \\
\hline TS-60-36-510 & 302701095331201 & 04-20-00 & $<.2$ & $<.2$ & $<.2$ & $<.2$ & $<.2$ & $<.2$ & $<.2$ & $<.05$ & $<2.5$ \\
\hline TW-22-02-714 & 335416100502101 & $05-05-00$ & $<.2$ & $<.2$ & .13 & $<.2$ & $<.2$ & $<.2$ & $<.2$ & 1.49 & 12.5 \\
\hline TW-22-02-716 & 335449100513101 & $12-19-00$ & $<.2$ & $<.2$ & $<.2$ & $<.2$ & $<.2$ & $<.2$ & $<.2$ & E.033 & 12.8 \\
\hline UJ-62-49-712 & 300906093585201 & 04-28-00 & $<.2$ & $<.2$ & $<.2$ & $<.2$ & $<.2$ & $<.2$ & $<.2$ & $<.05$ & $<2.5$ \\
\hline UK-31-05-501 & 325545098255501 & $03-22-00$ & $<.2$ & $<.2$ & .45 & $<.2$ & $<.2$ & $<.2$ & $<.2$ & 3.46 & 15.4 \\
\hline UK-31-24-501 & 324210098034701 & $03-22-00$ & $<.2$ & $<.2$ & $<.2$ & $<.2$ & $<.2$ & $<.2$ & $<.2$ & 5.63 & 13.8 \\
\hline UL-35-48-202 & 322207094034501 & $04-25-00$ & $<.2$ & $<.2$ & $<.2$ & $<.2$ & $<.2$ & $<.2$ & $<.2$ & $<.05$ & $<2.5$ \\
\hline UP-32-10-604 & 324926097455001 & 02-09-00 & $<.2$ & $<.2$ & $<.2$ & $<.2$ & $<.2$ & $<.2$ & $<.2$ & 7.56 & 12.8 \\
\hline UP-32-11-103 & 325005097440101 & $02-09-00$ & $<.2$ & $<.2$ & $<.2$ & $<.2$ & $<.2$ & $<.2$ & $<.2$ & 2.23 & 4.2 \\
\hline UP-32-11-709 & 324649097442001 & 02-09-00 & $<.2$ & $<.2$ & $<.2$ & $<.2$ & $<.2$ & $<.2$ & $<.2$ & .816 & 12.8 \\
\hline
\end{tabular}


Table 4. Concentrations of volatile organic compounds, nitrite plus nitrate, and tritium in public water-supply wells sampled in Texas, November 1999-January 2001-Continued

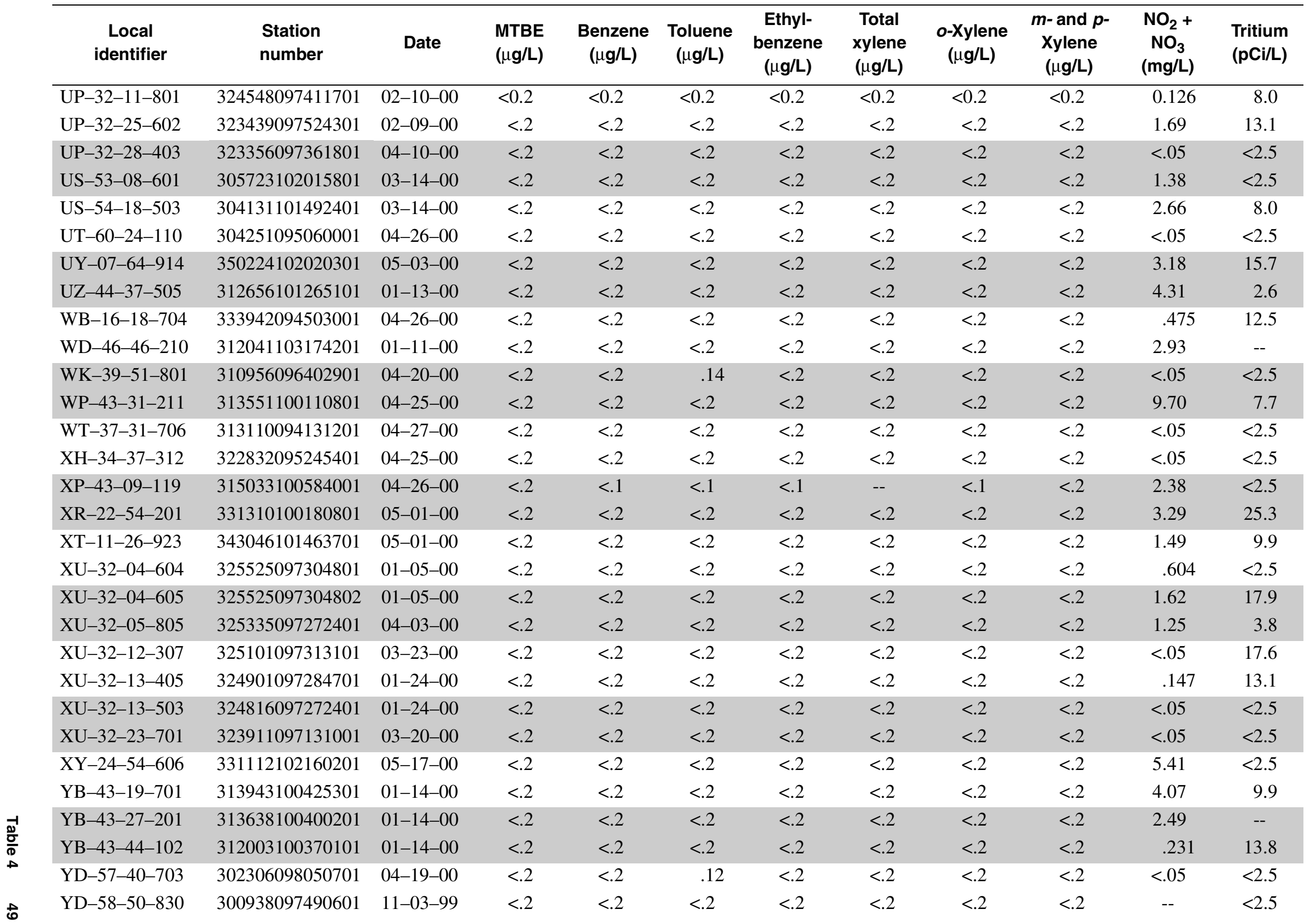


Table 4. Concentrations of volatile organic compounds, nitrite plus nitrate, and tritium in public water-supply wells sampled in Texas, November 1999-January 2001-Continued

\begin{tabular}{|c|c|c|c|c|c|c|c|c|c|c|c|}
\hline $\begin{array}{c}\text { Local } \\
\text { identifier }\end{array}$ & $\begin{array}{l}\text { Station } \\
\text { number }\end{array}$ & Date & $\begin{array}{l}\text { MTBE } \\
(\mu \mathrm{g} / L)\end{array}$ & $\begin{array}{c}\text { Benzene } \\
(\mu \mathrm{g} / \mathrm{L})\end{array}$ & $\begin{array}{c}\text { Toluene } \\
\text { ( } \mu \mathrm{g} / \mathrm{L})\end{array}$ & $\begin{array}{c}\text { Ethyl- } \\
\text { benzene } \\
(\mu \mathrm{g} / \mathrm{L})\end{array}$ & $\begin{array}{c}\text { Total } \\
\text { xylene } \\
(\mu \mathrm{g} / \mathrm{L})\end{array}$ & $\begin{array}{c}\text { o-Xylene } \\
\text { ( } \mu \mathrm{g} / \mathrm{L})\end{array}$ & $\begin{array}{c}m \text { - and } p \text { - } \\
\text { Xylene } \\
(\mu \mathrm{g} / \mathrm{L})\end{array}$ & $\begin{array}{c}\mathrm{NO}_{2}+ \\
\mathrm{NO}_{3} \\
(\mathrm{mg} / \mathrm{L})\end{array}$ & $\begin{array}{l}\text { Tritium } \\
\text { (pCi/L) }\end{array}$ \\
\hline YD-58-52-304 & 301305097314601 & $04-27-00$ & E0.12 & $<0.2$ & $<0.2$ & $<0.2$ & $<0.2$ & $<0.2$ & $<0.2$ & 3.26 & 11.2 \\
\hline YH-60-13-308 & 305125095225701 & 01-19-01 & $<.2$ & $<.2$ & $<.2$ & $<.2$ & $<.2$ & $<.2$ & $<.2$ & .218 & 13.8 \\
\hline YP-69-27-107 & 293652099442501 & 10-19-00 & $<.2$ & $<.2$ & $<.2$ & $<.2$ & $<.2$ & $<.2$ & $<.2$ & .334 & 9.6 \\
\hline YP-69-27-402 & 293450099433701 & $10-19-00$ & $<.2$ & $<.2$ & $<.2$ & $<.2$ & $<.2$ & $<.2$ & $<.2$ & .205 & 9.0 \\
\hline YP-69-27-704 & 293037099430301 & $10-18-00$ & $<.2$ & $<.2$ & $<.2$ & $<.2$ & $<.2$ & $<.2$ & $<.2$ & 1.67 & 9.9 \\
\hline YP-69-28-303 & 293644099314601 & $10-18-00$ & $<.2$ & $<.2$ & $<.2$ & $<.2$ & $<.2$ & $<.2$ & $<.2$ & .081 & 7.4 \\
\hline YT-79-24-101 & 284431097071801 & 02-29-00 & $<.2$ & $<.2$ & $<.2$ & $<.2$ & $<.2$ & $<.2$ & $<.2$ & .281 & $<2.5$ \\
\hline$Y X-46-32-625$ & 313252103010301 & $01-11-00$ & $<.2$ & $<.2$ & $<.2$ & $<.2$ & $<.2$ & $<.2$ & $<.2$ & 1.15 & $<2.5$ \\
\hline YX-46-40-311 & 312848103012901 & $01-11-00$ & $<.2$ & $<.2$ & $<.2$ & $<.2$ & $<.2$ & $<.2$ & $<.2$ & 1.06 & $<2.5$ \\
\hline YY-59-54-902 & 300824096152400 & 04-19-00 & $<.2$ & $<.2$ & $<.2$ & $<.2$ & $<.2$ & $<.2$ & $<.2$ & $<.05$ & $<2.5$ \\
\hline ZB-05-29-819 & 353215100261201 & $05-10-00$ & $<.2$ & $<.2$ & .11 & $<.2$ & $<.2$ & $<.2$ & $<.2$ & 2.75 & 2.9 \\
\hline ZB-05-38-512 & 352709100182901 & $05-08-00$ & $<.2$ & $<.2$ & $<.2$ & $<.2$ & $<.2$ & $<.2$ & $<.2$ & 12.6 & 8.6 \\
\hline $\mathrm{ZH}-13-46-414$ & 341848099222501 & $05-09-00$ & $<.2$ & $<.2$ & $<.2$ & $<.2$ & $<.2$ & $<.2$ & $<.2$ & 14.2 & 34.6 \\
\hline $\mathrm{ZH}-13-61-620$ & 340451099224401 & $12-19-00$ & $<.2$ & $<.2$ & $<.2$ & $<.2$ & $<.2$ & $<.2$ & $<.2$ & 23.0 & 19.2 \\
\hline ZK-58-19-620 & 304151097390301 & $03-30-00$ & $<.2$ & $<.2$ & $<.2$ & $<.2$ & $<.2$ & $<.2$ & $<.2$ & 2.30 & 11.8 \\
\hline ZK-58-19-804 & 303809097404701 & $03-30-00$ & $<.2$ & $<.2$ & $<.2$ & $<.2$ & $<.2$ & $<.2$ & $<.2$ & 3.28 & 9.6 \\
\hline ZK-58-19-805 & 303859097400901 & $03-30-00$ & $<.2$ & $<.2$ & $<.2$ & $<.2$ & $<.2$ & $<.2$ & $<.2$ & 3.32 & 11.2 \\
\hline ZK-58-27-819 & 303117097421301 & $04-18-00$ & $<.2$ & $<.2$ & $<.2$ & $<.2$ & $<.2$ & $<.2$ & $<.2$ & 1.78 & 10.6 \\
\hline $\mathrm{ZP}-46-16-102$ & 315042103054301 & $01-12-00$ & $<.2$ & $<.2$ & $<.2$ & $<.2$ & $<.2$ & $<.2$ & $<.2$ & 7.22 & -- \\
\hline ZR-19-42-612 & 331902097472301 & 04-04-00 & $<.2$ & $<.2$ & $<.2$ & $<.2$ & $<.2$ & $<.2$ & $<.2$ & 2.08 & $<2.5$ \\
\hline ZR-19-51-801 & 330906097411701 & 04-04-00 & $<.2$ & $<.2$ & $<.2$ & $<.2$ & $<.2$ & $<.2$ & $<.2$ & 11.2 & 6.4 \\
\hline ZR-19-51-805 & 330839097412401 & 04-04-00 & $<.2$ & $<.2$ & $<.2$ & $<.2$ & $<.2$ & $<.2$ & $<.2$ & .360 & $<2.5$ \\
\hline ZR-19-61-705 & 330014097285401 & $01-25-00$ & $<.2$ & $<.2$ & $<.2$ & $<.2$ & $<.2$ & $<.2$ & $<.2$ & .337 & 3.8 \\
\hline ZT-24-50-403 & 331051102511901 & $05-17-00$ & $<.2$ & $<.2$ & $<.2$ & $<.2$ & $<.2$ & $<.2$ & $<.2$ & 3.21 & 4.5 \\
\hline ZT-27-02-103 & 325819102501601 & $05-23-00$ & $<.2$ & $<.2$ & $<.2$ & $<.2$ & $<.2$ & $<.2$ & $<.2$ & 6.48 & 6.4 \\
\hline ZT-27-02-105 & 325819102503701 & $05-23-00$ & $<.2$ & $<.2$ & $<.2$ & $<.2$ & $<.2$ & $<.2$ & $<.2$ & .747 & -- \\
\hline
\end{tabular}


Table 5. Concentrations of soluble pesticides in public water-supply wells sampled in Texas, November 1999-January 2001

$[\mu \mathrm{g} / \mathrm{L}$, micrograms per liter; <, less than; E, estimated; --, not analyzed]

\begin{tabular}{|c|c|c|c|c|c|c|c|c|c|c|c|}
\hline Local identifier & $\begin{array}{l}\text { Station } \\
\text { number }\end{array}$ & Date & $\begin{array}{l}\text { Aceto- } \\
\text { chlor } \\
(\mu \mathrm{g} / \mathrm{L})\end{array}$ & $\begin{array}{c}\text { Aci- } \\
\text { fluorfen } \\
(\mu \mathrm{g} / \mathrm{L})\end{array}$ & $\begin{array}{l}\text { Ala- } \\
\text { chlor } \\
(\mu \mathrm{g} / \mathrm{L})\end{array}$ & $\begin{array}{c}\text { Aldi- } \\
\text { carb } \\
(\mu \mathrm{g} / \mathrm{L})\end{array}$ & $\begin{array}{l}\text { Aldicarb } \\
\text { sulfone } \\
(\mu \mathrm{g} / \mathrm{L})\end{array}$ & $\begin{array}{c}\text { Aldicarb } \\
\text { sulfoxide } \\
(\mu \mathrm{g} / \mathrm{L})\end{array}$ & $\begin{array}{l}\text { alpha- } \\
\text { BHC } \\
(\mu \mathrm{g} / \mathrm{L})\end{array}$ & $\begin{array}{l}\text { Atra- } \\
\text { zine } \\
(\mu \mathrm{g} / \mathrm{L})\end{array}$ & $\begin{array}{c}\text { Azinphos- } \\
\text { methyl } \\
(\mu \mathrm{g} / \mathrm{L})\end{array}$ \\
\hline AB-27-37-103 & 322805102283801 & $05-17-00$ & $<0.002$ & $<0.09$ & $<0.002$ & $<0.21$ & $<0.1$ & $<0.021$ & $<0.002$ & $<0.001$ & $<0.001$ \\
\hline $\mathrm{AB}-27-37-105$ & 322826102280101 & $05-17-00$ & $<.002$ & $<.09$ & $<.002$ & $<.21$ & $<.1$ & $<.021$ & $<.002$ & $<.001$ & $<.001$ \\
\hline AK-06-52-506 & 351024101334401 & 05-04-00 & $<.002$ & $<.09$ & $<.002$ & $<61.3$ & $<.24$ & $<.021$ & $<.002$ & $<.001$ & $<.001$ \\
\hline AP-59-62-403 & 300259096195001 & 04-03-00 & $<.002$ & $<.09$ & $<.002$ & $<.21$ & $<.12$ & $<.021$ & $<.002$ & $<.001$ & $<.001$ \\
\hline AP-66-16-407 & 294903096061401 & $04-21-00$ & $<.002$ & $<.09$ & $<.002$ & $<.21$ & $<.1$ & $<.021$ & $<.002$ & $<.001$ & $<.001$ \\
\hline AR-10-51-408 & 341229102435401 & 05-01-00 & $<.002$ & $<.09$ & $<.002$ & $<.35$ & $<.1$ & $<.021$ & $<.002$ & $<.001$ & $<.001$ \\
\hline AR-24-09-604 & 335000102545701 & 05-09-00 & $<.002$ & $<.09$ & $<.002$ & $<.6$ & $<.125$ & $<.021$ & $<.002$ & $<.001$ & $<.001$ \\
\hline AT-58-62-206 & 300646097193001 & 12-10-99 & $<.002$ & $<.09$ & $<.002$ & $<.21$ & $<.1$ & $<.021$ & $<.002$ & .019 & $<.001$ \\
\hline AU-21-30-389 & 333612099163101 & $05-02-00$ & $<.002$ & $<.09$ & $<.002$ & $<.21$ & $<.1$ & $<.021$ & $<.002$ & $<.001$ & $<.001$ \\
\hline$A X-58-04-604$ & 305718097314401 & $03-22-00$ & $<.002$ & $<.09$ & $<.002$ & $<.21$ & $<.1$ & $<.15$ & $<.002$ & .015 & $<.001$ \\
\hline $\mathrm{AX}-58-04-623$ & 305624097321101 & $03-22-00$ & $<.002$ & $<.09$ & $<.002$ & $<.4$ & $<.1$ & $<.021$ & $<.002$ & $<.001$ & $<.001$ \\
\hline AY-68-35-105 & 292842098425001 & 03-06-00 & $<.002$ & $<.09$ & $<.002$ & $<.21$ & $<.1$ & $<.021$ & $<.002$ & $<.001$ & $<.01$ \\
\hline AZ-57-45-612 & 301923098225401 & 04-18-00 & $<.002$ & $<.09$ & $<.002$ & $<.54$ & $<.1$ & $<.021$ & $<.002$ & $<.001$ & $<.001$ \\
\hline AZ-57-45-811 & 301633098252901 & 03-29-00 & $<.002$ & $<.09$ & $<.002$ & $<.4$ & $<.1$ & $<.021$ & $<.002$ & $<.001$ & $<.001$ \\
\hline BD-16-40-709 & 332301094061701 & 01-18-01 & $<.0041$ & $<.05$ & $<.0024$ & $<.21$ & $<.26$ & $<.021$ & $<.0046$ & $<.007$ & $<.05$ \\
\hline BK-73-47-504 & 291911103124301 & $12-28-00$ & $<.0041$ & $<.05$ & $<.0024$ & $<.21$ & $<.26$ & $<.021$ & $<.0046$ & $<.007$ & $<.05$ \\
\hline BK-73-52-905 & 290819103312601 & $12-28-00$ & $<.0041$ & $<.05$ & $<.0024$ & $<.21$ & $<.26$ & $<.021$ & $<.0046$ & $<.007$ & $<.05$ \\
\hline BL-11-38-334 & 342841101155301 & $05-01-00$ & $<.002$ & $<.09$ & $<.002$ & $<.31$ & $<.1$ & $<.021$ & $<.002$ & $<.001$ & $<.001$ \\
\hline BL-11-48-507 & 341919101035201 & $05-02-00$ & $<.002$ & $<.09$ & $<.002$ & $<.21$ & $<.1$ & $<.021$ & $<.002$ & $<.001$ & $<.001$ \\
\hline BL-12-41-204 & 342109100570301 & $05-02-00$ & $<.002$ & $<.09$ & $<.002$ & $<.21$ & $<.1$ & $<.021$ & $<.002$ & $<.001$ & $<.001$ \\
\hline BR-41-01-244 & 315849098552101 & 04-05-00 & $<.002$ & $<.09$ & $<.002$ & $<.21$ & $<.1$ & $<.021$ & $<.002$ & $<.001$ & $<.001$ \\
\hline BT-57-14-403 & 304939098202401 & 03-08-00 & $<.002$ & $<.09$ & $<.002$ & $<.39$ & $<.1$ & $<.021$ & $<.002$ & $<.001$ & $<.01$ \\
\hline BT-57-14-404 & 304927098211201 & 03-08-00 & $<.002$ & $<.09$ & $<.002$ & $<.21$ & $<.1$ & $<.21$ & $<.002$ & $<.001$ & $<.001$ \\
\hline BT-57-21-607 & 304125098224001 & $03-28-00$ & $<.002$ & $<.09$ & $<.002$ & $<.35$ & $<.1$ & $<.021$ & $<.002$ & $<.001$ & $<.001$ \\
\hline BT-57-21-608 & 304121098224201 & $03-28-00$ & $<.002$ & $<.09$ & $<.002$ & $<.24$ & $<.1$ & $<.021$ & $<.002$ & $<.001$ & $<.001$ \\
\hline BT $-57-23-110$ & 304406098130801 & 03-29-00 & $<.002$ & $<.09$ & $<.002$ & $<.58$ & $<.1$ & $<.021$ & $<.002$ & .005 & $<.001$ \\
\hline BT-57-30-101 & 303548098211701 & 03-01-00 & $<.002$ & $<.09$ & $<.002$ & $<.42$ & $<.1$ & $<.021$ & $<.002$ & .017 & $<.01$ \\
\hline BU-67-10-910 & 294528097464401 & $03-21-00$ & $<.002$ & $<.09$ & .006 & $<.76$ & $<.1$ & $<.021$ & $<.002$ & .414 & $<.001$ \\
\hline
\end{tabular}


Table 5. Concentrations of soluble pesticides in public water-supply wells sampled in Texas, November 1999-January 2001-Continued

\begin{tabular}{|c|c|c|c|c|c|c|c|c|c|c|c|}
\hline Local identifier & $\begin{array}{l}\text { Station } \\
\text { number }\end{array}$ & Date & $\begin{array}{l}\text { Aceto- } \\
\text { chlor } \\
(\mu \mathrm{g} / \mathrm{L})\end{array}$ & $\begin{array}{c}\text { Aci- } \\
\text { fluorfen } \\
(\mu \mathrm{g} / \mathrm{L})\end{array}$ & $\begin{array}{l}\text { Ala- } \\
\text { chlor } \\
(\mu \mathrm{g} / \mathrm{L})\end{array}$ & $\begin{array}{c}\text { Aldi- } \\
\text { carb } \\
(\mu \mathrm{g} / \mathrm{L})\end{array}$ & $\begin{array}{c}\text { Aldicarb } \\
\text { sulfone } \\
(\mu \mathrm{g} / \mathrm{L})\end{array}$ & $\begin{array}{c}\text { Aldicarb } \\
\text { sulfoxide } \\
(\mu \mathrm{g} / \mathrm{L})\end{array}$ & $\begin{array}{c}\text { alpha- } \\
\text { BHC } \\
(\mu \mathrm{g} / \mathrm{L})\end{array}$ & $\begin{array}{c}\text { Atra- } \\
\text { zine } \\
(\mu \mathrm{g} / \mathrm{L})\end{array}$ & $\begin{array}{c}\text { Azinphos- } \\
\text { methyl } \\
(\mu \mathrm{g} / L)\end{array}$ \\
\hline BX-30-55-936 & 320939099093801 & $12-18-00$ & $<0.0041$ & $<0.05$ & $<0.0024$ & $<0.21$ & $<0.2$ & $<0.021$ & $<0.0046$ & $<0.007$ & $<0.05$ \\
\hline DA-06-28-202 & 353704101343701 & $05-03-00$ & $<.002$ & $<.09$ & $<.002$ & $<.21$ & $<.1$ & $<.021$ & $<.002$ & $<.001$ & $<.001$ \\
\hline DL-14-62-604 & 340406098160701 & $05-10-00$ & $<.002$ & $<.09$ & $<.002$ & $<.27$ & $<.1$ & $<.021$ & $<.002$ & $<.001$ & $<.001$ \\
\hline DP-24-18-308 & 334352102450601 & 05-09-00 & $<.002$ & $<.09$ & $<.002$ & $<.88$ & $<.1$ & $<.021$ & $<.002$ & $<.001$ & $<.001$ \\
\hline DP-25-24-304 & 334245103011001 & $05-08-00$ & $<.002$ & $<.09$ & $<.002$ & $<.59$ & $<.1$ & $<.021$ & $<.002$ & $<.001$ & $<.001$ \\
\hline DU-12-06-609 & 345658100155501 & $05-08-00$ & $<.002$ & $<.09$ & $<.002$ & $<.21$ & $<.1$ & $<.021$ & $<.002$ & $<.001$ & $<.001$ \\
\hline DX-68-05-621 & 295520098242301 & $03-22-00$ & $<.002$ & $<.09$ & $<.002$ & $<.21$ & $<.1$ & $<.021$ & $<.002$ & $<.001$ & $<.001$ \\
\hline DX-68-06-405 & 295528098222301 & $03-22-00$ & $<.002$ & $<.09$ & $<.002$ & $<.41$ & $<.1$ & $<.021$ & $<.002$ & $<.001$ & $<.001$ \\
\hline DY-41-13-307 & 315105098241401 & $04-05-00$ & $<.002$ & $<.09$ & $<.002$ & $<.21$ & $<.1$ & $<.021$ & $<.002$ & E.002 & $<.001$ \\
\hline $\mathrm{HH}-45-35-505$ & 312715102402201 & $01-12-00$ & $<.002$ & $<.09$ & $<.002$ & $<.71$ & $<.1$ & $<.021$ & $<.002$ & $<.001$ & $<.001$ \\
\hline HP-12-62-802 & 340210100184901 & $05-05-00$ & $<.002$ & $<.09$ & $<.002$ & -- & -- & -- & $<.002$ & .009 & $<.001$ \\
\hline HS-28-09-404 & 324810101591201 & $05-16-00$ & $<.002$ & $<.09$ & $<.002$ & $<.21$ & $<.1$ & $<.021$ & $<.002$ & $<.001$ & $<.001$ \\
\hline HT-07-63-204 & 350607102103401 & $05-03-00$ & $<.002$ & $<.09$ & $<.002$ & $<.66$ & $<.1$ & $<.021$ & $<.002$ & $<.001$ & $<.001$ \\
\hline HZ-77-34-606 & 282608099455301 & $03-28-00$ & $<.002$ & $<.09$ & $<.002$ & $<.76$ & $<.1$ & $<.021$ & $<.002$ & $<.001$ & $<.001$ \\
\hline JA-05-57-613 & 350254100535501 & 05-04-00 & $<.002$ & $<.09$ & $<.002$ & $<.21$ & $<.1$ & $<.021$ & $<.002$ & $<.001$ & $<.001$ \\
\hline JA-12-12-404 & 344910100360401 & $05-02-00$ & $<.002$ & $<.09$ & $<.002$ & $<.21$ & $<.1$ & $<.021$ & $<.002$ & $<.001$ & $<.001$ \\
\hline JH-45-05-924 & 315245102240201 & $05-25-00$ & $<.002$ & $<.09$ & $<.002$ & $<.21$ & $<.1$ & $<.021$ & $<.002$ & $<.001$ & $<.001$ \\
\hline JL-49-04-116 & 315757106370201 & $01-06-00$ & $<.002$ & $<.09$ & $<.002$ & $<.22$ & $<.1$ & $<.021$ & $<.002$ & $<.001$ & $<.001$ \\
\hline JL-49-04-423 & 315708106362301 & $01-03-00$ & $<.002$ & $<.09$ & $<.002$ & $<.21$ & $<.1$ & $<.021$ & $<.002$ & $<.001$ & $<.001$ \\
\hline JL-49-04-428 & 315517106361401 & $01-06-00$ & $<.002$ & $<.09$ & $<.002$ & $<.76$ & $<.1$ & $<.021$ & $<.002$ & $<.001$ & $<.001$ \\
\hline JL-49-04-492 & 315623106360601 & $01-06-00$ & $<.002$ & $<.09$ & $<.002$ & $<.4$ & $<.1$ & $<.021$ & $<.002$ & $<.001$ & $<.001$ \\
\hline JL-49-22-626 & 314145106163601 & 01-04-00 & $<.002$ & $<.09$ & $<.002$ & $<.53$ & $<.1$ & $<.021$ & $<.002$ & $<.001$ & $<.001$ \\
\hline JL-49-24-423 & 314107106063301 & $01-07-00$ & $<.002$ & $<.09$ & $<.002$ & $<.47$ & $<.1$ & $<.021$ & $<.002$ & $<.001$ & $<.001$ \\
\hline JL-49-40-502 & 312648106044701 & 01-04-00 & $<.002$ & $<.09$ & $<.002$ & $<.36$ & $<.1$ & $<.021$ & $<.002$ & $<.001$ & $<.001$ \\
\hline JY-65-26-520 & 293314095474702 & 04-03-00 & $<.002$ & $<.09$ & $<.002$ & $<.21$ & $<.1$ & $<.021$ & $<.002$ & $<.001$ & $<.001$ \\
\hline KD-27-01-612 & 325523102542701 & $05-22-00$ & $<.002$ & $<.09$ & $<.002$ & $<.21$ & $<.1$ & $<.021$ & $<.002$ & $<.001$ & $<.001$ \\
\hline KK-57-42-709 & 301545098502801 & 07-06-00 & $<.002$ & $<.09$ & $<.002$ & $<.21$ & $<.1$ & $<.021$ & $<.002$ & $<.001$ & $<.001$ \\
\hline $\mathrm{KK}-57-50-325$ & 301357098471901 & $03-28-00$ & $<.002$ & $<.09$ & $<.002$ & $<.21$ & $<.1$ & $<.021$ & $<.002$ & .006 & $<.001$ \\
\hline $\mathrm{KK}-57-51-303$ & 301411098383101 & $03-21-00$ & $<.002$ & $<.09$ & $<.002$ & $<.62$ & $<.1$ & $<.16$ & $<.002$ & .019 & $<.001$ \\
\hline KK-57-52-107 & 301430098363401 & $03-21-00$ & $<.002$ & $<.09$ & $<.002$ & $<.21$ & $<.1$ & $<.021$ & $<.002$ & $<.001$ & $<.001$ \\
\hline
\end{tabular}


Table 5. Concentrations of soluble pesticides in public water-supply wells sampled in Texas, November 1999-January 2001-Continued

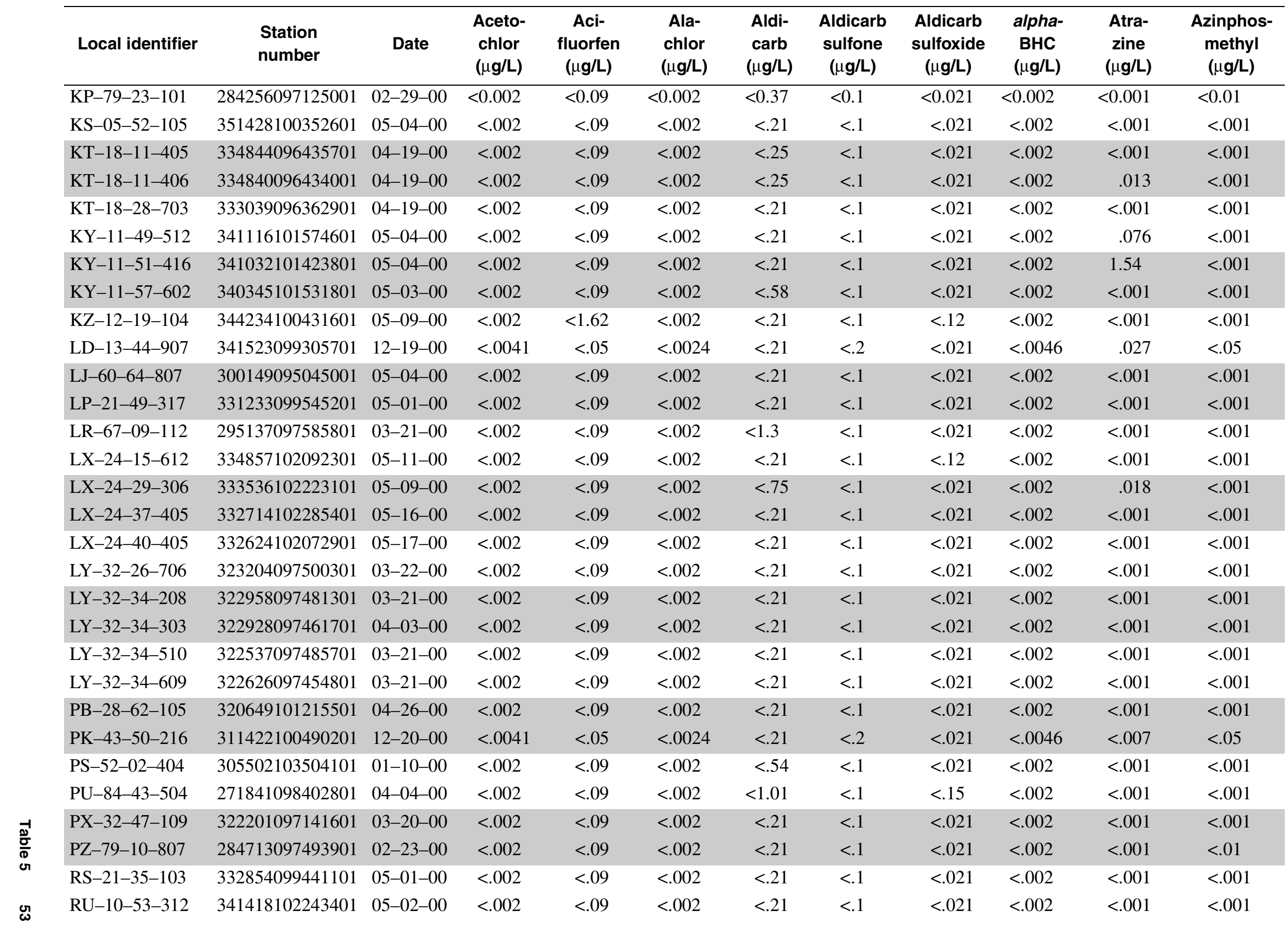


Table 5. Concentrations of soluble pesticides in public water-supply wells sampled in Texas, November 1999-January 2001-Continued

\begin{tabular}{|c|c|c|c|c|c|c|c|c|c|c|c|}
\hline Local identifier & $\begin{array}{l}\text { Station } \\
\text { number }\end{array}$ & Date & $\begin{array}{c}\text { Aceto- } \\
\text { chlor } \\
(\mu \mathrm{g} / \mathrm{L})\end{array}$ & $\begin{array}{c}\text { Aci- } \\
\text { fluorfen } \\
(\mu \mathrm{g} / \mathrm{L})\end{array}$ & $\begin{array}{l}\text { Ala- } \\
\text { chlor } \\
(\mu \mathrm{g} / \mathrm{L})\end{array}$ & $\begin{array}{c}\text { Aldi- } \\
\text { carb } \\
(\mu \mathrm{g} / \mathrm{L})\end{array}$ & $\begin{array}{c}\text { Aldicarb } \\
\text { sulfone } \\
\text { ( } \mu \mathrm{g} / \mathrm{L})\end{array}$ & $\begin{array}{c}\text { Aldicarb } \\
\text { sulfoxide } \\
(\mu \mathrm{g} / \mathrm{L})\end{array}$ & $\begin{array}{c}\text { alpha- } \\
\text { BHC } \\
(\mu \mathrm{g} / \mathrm{L})\end{array}$ & $\begin{array}{c}\text { Atra- } \\
\text { zine } \\
(\mu \mathrm{g} / \mathrm{L})\end{array}$ & $\begin{array}{c}\text { Azinphos- } \\
\text { methyl } \\
(\mu \mathrm{g} / \mathrm{L})\end{array}$ \\
\hline RU-10-60-606 & 340427102302801 & $05-02-00$ & $<0.002$ & $<0.09$ & $<0.002$ & $<0.56$ & $<0.1$ & $<0.021$ & $<0.002$ & $<0.001$ & $<0.001$ \\
\hline RW-41-63-520 & 310238098104001 & $03-23-00$ & $<.002$ & $<.09$ & $<.002$ & $<.68$ & $<.1$ & $<.13$ & $<.002$ & $<.001$ & $<.001$ \\
\hline SK-57-19-201 & 304323098421701 & $03-08-00$ & $<.002$ & $<.09$ & $<.002$ & $<.86$ & $<.1$ & $<.021$ & $<.002$ & $<.001$ & $<.01$ \\
\hline SK-57-34-201 & 303001098485201 & 03-09-00 & $<.002$ & $<.09$ & $<.002$ & $<.21$ & $<.1$ & $<.021$ & $<.002$ & $<.001$ & $<.001$ \\
\hline SP-23-17-417 & 334043101585701 & $05-10-00$ & $<.002$ & $<.09$ & $<.002$ & $<.21$ & $<.1$ & $<.021$ & $<.002$ & .017 & $<.001$ \\
\hline SP-23-18-107 & 334306101504101 & 05-10-00 & $<.002$ & $<.09$ & $<.002$ & $<.21$ & $<.1$ & $<.19$ & $<.002$ & .029 & $<.001$ \\
\hline SP-23-19-812 & 333955101404601 & $05-10-00$ & $<.002$ & $<.09$ & $<.002$ & $<.42$ & $<.1$ & $<.021$ & $<.002$ & $<.001$ & $<.001$ \\
\hline $\mathrm{SP}-24-24-214$ & 334419102045201 & $05-18-00$ & $<.002$ & $<.09$ & $<.002$ & $<.21$ & $<.1$ & $<.021$ & $<.002$ & $<.001$ & $<.001$ \\
\hline SR-23-41-602 & 331933101543701 & $05-15-00$ & $<.002$ & $<.09$ & $<.002$ & $<.22$ & $<308.36$ & $<.021$ & $<.002$ & .215 & $<.001$ \\
\hline SS-42-52-504 & 311202099321401 & $03-01-00$ & $<.002$ & $<.09$ & $<.002$ & $<.21$ & $<.1$ & $<.021$ & $<.002$ & $<.001$ & $<.01$ \\
\hline ST-40-39-304 & 312745097083301 & $03-23-00$ & $<.002$ & $<.09$ & $<.002$ & $<.36$ & $<.1$ & $<.021$ & $<.002$ & $<.001$ & $<.001$ \\
\hline SY-27-39-505 & 322555102105501 & $05-24-00$ & $<.002$ & $<.09$ & $<.002$ & $<.44$ & $<.1$ & $<.16$ & $<.002$ & $<.001$ & $<.001$ \\
\hline SY-28-42-803 & 321619101495001 & $05-23-00$ & $<.002$ & $<.09$ & $<.002$ & $<.21$ & $<.1$ & $<.021$ & $<.002$ & $<.001$ & $<.001$ \\
\hline SY-28-50-908 & 320816101474601 & $05-23-00$ & $<.002$ & $<.09$ & $<.002$ & $<.21$ & $<.1$ & $<.09$ & $<.002$ & $<.001$ & $<.001$ \\
\hline TB-76-03-605 & 285657100373801 & $03-28-00$ & $<.002$ & $<.09$ & $<.002$ & $<.23$ & $<.13$ & $<.021$ & $<.002$ & $<.001$ & $<.001$ \\
\hline TJ-27-63-712 & 320143102123101 & $12-20-00$ & $<.0041$ & $<.05$ & $<.0024$ & $<.21$ & $<.2$ & $<.021$ & $<.0046$ & $<.007$ & $<.05$ \\
\hline TJ-28-57-702 & 320221101594901 & $05-25-00$ & $<.002$ & $<.09$ & $<.002$ & $<.21$ & $<.1$ & $<.021$ & $<.002$ & $<.001$ & $<.001$ \\
\hline TJ-28-57-903 & 320010101523701 & $05-23-00$ & $<.002$ & $<.09$ & $<.002$ & $<.21$ & $<.1$ & $<.021$ & $<.002$ & $<.001$ & $<.001$ \\
\hline TJ-45-07-407 & 315643102131101 & $05-24-00$ & $<.002$ & $<.09$ & $<.002$ & $<.21$ & $<.1$ & $<.021$ & $<.002$ & $<.001$ & $<.001$ \\
\hline TJ-45-07-606 & 315702102075401 & $05-25-00$ & $<.002$ & $<.09$ & $<.002$ & $<.21$ & $<.1$ & $<.021$ & $<.002$ & $<.001$ & $<.001$ \\
\hline TS-60-36-510 & 302701095331201 & 04-20-00 & $<.002$ & $<.09$ & $<.002$ & $<.21$ & $<.1$ & $<.021$ & $<.002$ & $<.001$ & $<.001$ \\
\hline TW-22-02-714 & 335416100502101 & $05-05-00$ & $<.002$ & $<.09$ & $<.002$ & $<.21$ & $<.1$ & $<.021$ & $<.002$ & $<.001$ & $<.001$ \\
\hline TW-22-02-716 & 335449100513101 & $12-19-00$ & $<.0041$ & $<.05$ & $<.0024$ & $<.21$ & $<.2$ & $<.021$ & $<.0046$ & $<.007$ & $<.05$ \\
\hline UJ-62-49-712 & 300906093585201 & $04-28-00$ & $<.002$ & $<.09$ & $<.002$ & $<.21$ & $<.1$ & $<.021$ & $<.002$ & $<.001$ & $<.001$ \\
\hline UK-31-05-501 & 325545098255501 & $03-22-00$ & $<.002$ & $<.09$ & $<.002$ & $<.21$ & $<.1$ & $<.12$ & $<.002$ & .006 & $<.001$ \\
\hline UK-31-24-501 & 324210098034701 & $03-22-00$ & $<.002$ & $<.09$ & $<.002$ & $<.33$ & $<.1$ & $<.021$ & $<.002$ & $<.001$ & $<.001$ \\
\hline UL-35-48-202 & 322207094034501 & $04-25-00$ & $<.002$ & $<.09$ & $<.002$ & $<.21$ & $<.1$ & $<.021$ & $<.002$ & $<.001$ & $<.001$ \\
\hline UP-32-10-604 & 324926097455001 & 02-09-00 & $<.002$ & $<.09$ & $<.002$ & $<.21$ & $<.1$ & $<.021$ & $<.002$ & $<.001$ & $<.001$ \\
\hline UP-32-11-103 & 325005097440101 & 02-09-00 & $<.002$ & $<.09$ & $<.002$ & $<.21$ & $<.1$ & $<.021$ & $<.002$ & $<.001$ & $<.001$ \\
\hline UP-32-11-709 & 324649097442001 & 02-09-00 & $<.002$ & $<.09$ & $<.002$ & $<1.87$ & $<.1$ & $<.021$ & $<.002$ & $<.001$ & $<.001$ \\
\hline
\end{tabular}


Table 5. Concentrations of soluble pesticides in public water-supply wells sampled in Texas, November 1999-January 2001-Continued

\begin{tabular}{|c|c|c|c|c|c|c|c|c|c|c|c|}
\hline Local identifier & $\begin{array}{l}\text { Station } \\
\text { number }\end{array}$ & Date & $\begin{array}{l}\text { Aceto- } \\
\text { chlor } \\
(\mu \mathrm{g} / \mathrm{L})\end{array}$ & $\begin{array}{c}\text { Aci- } \\
\text { fluorfen } \\
(\mu \mathrm{g} / \mathrm{L})\end{array}$ & $\begin{array}{l}\text { Ala- } \\
\text { chlor } \\
(\mu \mathrm{g} / \mathrm{L})\end{array}$ & $\begin{array}{l}\text { Aldi- } \\
\text { carb } \\
(\mu \mathrm{g} / \mathrm{L})\end{array}$ & $\begin{array}{l}\text { Aldicarb } \\
\text { sulfone } \\
(\mu \mathrm{g} / \mathrm{L})\end{array}$ & $\begin{array}{c}\text { Aldicarb } \\
\text { sulfoxide } \\
(\mu \mathrm{g} / \mathrm{L})\end{array}$ & $\begin{array}{l}\text { alpha- } \\
\text { BHC } \\
(\mu \mathrm{g} / \mathrm{L})\end{array}$ & $\begin{array}{l}\text { Atra- } \\
\text { zine } \\
(\mu \mathrm{g} / \mathrm{L})\end{array}$ & $\begin{array}{c}\text { Azinphos- } \\
\text { methyl } \\
(\mu \mathrm{g} / \mathrm{L})\end{array}$ \\
\hline UP-32-11-801 & 324548097411701 & $02-10-00$ & $<0.002$ & $<0.09$ & $<0.002$ & $<0.31$ & $<0.1$ & $<0.021$ & $<0.002$ & $<0.001$ & $<0.001$ \\
\hline UP-32-28-403 & 323356097361801 & 04-10-00 & $<.002$ & $<.09$ & $<.002$ & $<.21$ & $<.1$ & $<.021$ & $<.002$ & $<.001$ & $<.001$ \\
\hline US-53-08-601 & 305723102015801 & $03-14-00$ & $<.002$ & $<.53$ & $<.002$ & $<.21$ & $<.1$ & $<.021$ & $<.002$ & $<.001$ & $<.001$ \\
\hline US-54-18-503 & 304131101492401 & $03-14-00$ & $<.002$ & $<.09$ & $<.002$ & $<.33$ & $<.1$ & $<.11$ & $<.002$ & $<.001$ & $<.001$ \\
\hline UZ-44-37-505 & 312656101265101 & $01-13-00$ & $<.002$ & $<.09$ & $<.002$ & $<.24$ & $<.1$ & $<.021$ & $<.002$ & $<.001$ & $<.001$ \\
\hline WB-16-18-704 & 333942094503001 & $04-26-00$ & $<.002$ & $<.09$ & $<.002$ & $<.21$ & $<.1$ & $<.021$ & $<.002$ & $<.001$ & $<.001$ \\
\hline WD-46-46-210 & 312041103174201 & $01-11-00$ & $<.002$ & $<.09$ & $<.002$ & $<.37$ & $<.1$ & $<.021$ & $<.002$ & $<.001$ & $<.001$ \\
\hline WK-39-51-801 & 310956096402901 & $04-20-00$ & $<.002$ & $<.09$ & $<.002$ & $<.55$ & $<.1$ & $<.021$ & $<.002$ & $<.001$ & $<.001$ \\
\hline WP-43-31-211 & 313551100110801 & $04-25-00$ & $<.002$ & $<.09$ & $<.002$ & $<.21$ & $<.1$ & $<.021$ & $<.002$ & $<.001$ & $<.001$ \\
\hline XT-11-26-923 & 343046101463701 & $05-01-00$ & $<.002$ & $<.09$ & $<.002$ & $<.57$ & $<.1$ & $<.021$ & $<.002$ & .028 & $<.001$ \\
\hline XU-32-04-604 & 325525097304801 & $01-05-00$ & $<.002$ & $<.09$ & $<.002$ & $<.42$ & $<.1$ & $<.021$ & $<.002$ & $<.001$ & $<.001$ \\
\hline XU-32-04-605 & 325525097304802 & $01-05-00$ & $<.002$ & $<.09$ & $<.002$ & $<.21$ & $<.1$ & $<.021$ & $<.002$ & $<.001$ & $<.001$ \\
\hline XU-32-05-805 & 325335097272401 & 04-03-00 & $<.002$ & $<.09$ & $<.002$ & $<.29$ & $<.1$ & $<.021$ & $<.002$ & $<.001$ & $<.001$ \\
\hline XU-32-12-307 & 325101097313101 & $03-23-00$ & $<.002$ & $<.09$ & $<.002$ & $<.21$ & $<.1$ & $<.021$ & $<.002$ & $<.001$ & $<.001$ \\
\hline XU-32-13-405 & 324901097284701 & $01-24-00$ & $<.002$ & $<.09$ & $<.002$ & $<.21$ & $<.1$ & $<.021$ & $<.002$ & $<.001$ & $<.001$ \\
\hline XU-32-13-503 & 324816097272401 & $01-24-00$ & $<.002$ & $<.09$ & $<.002$ & $<.21$ & $<.1$ & $<.021$ & $<.002$ & $<.001$ & $<.001$ \\
\hline XU-32-23-701 & 323911097131001 & $03-20-00$ & $<.002$ & $<.09$ & $<.002$ & $<.21$ & $<.1$ & $<.021$ & $<.002$ & $<.001$ & $<.001$ \\
\hline XY-24-54-606 & 331112102160201 & $05-17-00$ & $<.002$ & $<.09$ & $<.002$ & $<.42$ & $<.1$ & $<.021$ & $<.002$ & $<.001$ & $<.001$ \\
\hline YB-43-19-701 & 313943100425301 & $01-14-00$ & $<.002$ & $<.09$ & $<.002$ & $<.4$ & $<.1$ & $<.021$ & $<.002$ & $<.001$ & $<.001$ \\
\hline YB-43-27-201 & 313638100400201 & $01-14-00$ & $<.002$ & $<.09$ & $<.002$ & $<.34$ & $<.1$ & $<.021$ & $<.002$ & $<.001$ & $<.001$ \\
\hline
\end{tabular}


Table 5. Concentrations of soluble pesticides in public water-supply wells sampled in Texas, November 1999-January 2001-Continued

\begin{tabular}{|c|c|c|c|c|c|c|c|c|c|c|c|}
\hline Local identifier & $\begin{array}{l}\text { Station } \\
\text { number }\end{array}$ & Date & $\begin{array}{l}\text { Aceto- } \\
\text { chlor } \\
(\mu \mathrm{g} / \mathrm{L})\end{array}$ & $\begin{array}{c}\text { Aci- } \\
\text { fluorfen } \\
(\mu \mathrm{g} / \mathrm{L})\end{array}$ & $\begin{array}{l}\text { Ala- } \\
\text { chlor } \\
(\mu \mathrm{g} / \mathrm{L})\end{array}$ & $\begin{array}{l}\text { Aldi- } \\
\text { carb } \\
(\mu \mathrm{g} / \mathrm{L})\end{array}$ & $\begin{array}{c}\text { Aldicarb } \\
\text { sulfone } \\
(\mu \mathrm{g} / \mathrm{L})\end{array}$ & $\begin{array}{c}\text { Aldicarb } \\
\text { sulfoxide } \\
(\mu \mathrm{g} / \mathrm{L})\end{array}$ & $\begin{array}{c}\text { alpha- } \\
\text { BHC } \\
(\mu \mathrm{g} / \mathrm{L})\end{array}$ & $\begin{array}{c}\text { Atra- } \\
\text { zine } \\
(\mu \mathrm{g} / \mathrm{L})\end{array}$ & $\begin{array}{c}\text { Azinphos- } \\
\text { methyl } \\
(\mu \mathrm{g} / \mathrm{L})\end{array}$ \\
\hline YD-58-52-304 & 301305097314601 & $04-27-00$ & $<0.002$ & $<0.09$ & $<0.002$ & $<0.21$ & $<0.31$ & $<0.021$ & $<0.002$ & 0.043 & $<0.001$ \\
\hline YH-60-13-308 & 305125095225701 & 01-19-01 & $<.0041$ & $<.05$ & $<.0024$ & $<.21$ & $<.26$ & $<.021$ & $<.0046$ & .034 & $<.05$ \\
\hline YP-69-27-107 & 293652099442501 & $10-19-00$ & $<.0041$ & $<.05$ & $<.0024$ & $<.21$ & $<.26$ & $<.021$ & $<.0046$ & $<.007$ & $<.05$ \\
\hline YP-69-27-402 & 293450099433701 & $10-19-00$ & $<.0041$ & $<.05$ & $<.0024$ & $<.21$ & $<.26$ & $<.021$ & $<.0046$ & $<.007$ & $<.05$ \\
\hline YP-69-27-704 & 293037099430301 & $10-18-00$ & $<.0041$ & $<.05$ & $<.0024$ & $<.21$ & $<.26$ & $<.021$ & $<.0046$ & $<.007$ & $<.05$ \\
\hline YP-69-28-303 & 293644099314601 & $10-18-00$ & $<.0041$ & $<.05$ & $<.0024$ & $<.21$ & $<.26$ & $<.021$ & $<.0046$ & $<.007$ & $<.05$ \\
\hline YT-79-24-101 & 284431097071801 & 02-29-00 & $<.002$ & $<.09$ & $<.002$ & $<.42$ & $<.1$ & $<.021$ & $<.002$ & $<.001$ & $<.01$ \\
\hline$Y X-46-32-625$ & 313252103010301 & $01-11-00$ & $<.002$ & $<.09$ & $<.002$ & $<.72$ & $<.1$ & $<.021$ & $<.002$ & $<.001$ & $<.001$ \\
\hline $\mathrm{YX}-46-40-311$ & 312848103012901 & $01-11-00$ & $<.002$ & $<.09$ & $<.002$ & $<.29$ & $<.1$ & $<.021$ & $<.002$ & $<.001$ & $<.001$ \\
\hline YY-59-54-902 & 300824096152400 & 04-19-00 & $<.002$ & $<.09$ & $<.002$ & $<.21$ & $<.1$ & $<.021$ & $<.002$ & $<.001$ & $<.001$ \\
\hline ZB-05-29-819 & 353215100261201 & $05-10-00$ & $<.002$ & $<.09$ & $<.002$ & $<.46$ & $<.1$ & $<.021$ & $<.002$ & $<.001$ & $<.001$ \\
\hline ZB-05-38-512 & 352709100182901 & $05-08-00$ & $<.002$ & $<.09$ & $<.002$ & $<.29$ & $<.1$ & $<.021$ & $<.002$ & .009 & $<.001$ \\
\hline $\mathrm{ZH}-13-46-414$ & 341848099222501 & 05-09-00 & $<.002$ & $<.09$ & $<.002$ & $<.8$ & $<.16$ & $<.021$ & $<.002$ & $<.001$ & $<.001$ \\
\hline $\mathrm{ZH}-13-61-620$ & 340451099224401 & $12-19-00$ & $<.0041$ & $<.05$ & $<.0024$ & $<.21$ & $<.2$ & $<.021$ & $<.0046$ & $<.007$ & $<.05$ \\
\hline ZK-58-19-620 & 304151097390301 & $03-30-00$ & $<.002$ & $<.09$ & $<.002$ & $<.85$ & $<.1$ & $<.021$ & $<.002$ & .005 & $<.001$ \\
\hline ZK-58-19-804 & 303809097404701 & $03-30-00$ & $<.002$ & $<.09$ & $<.002$ & $<.3$ & $<.1$ & $<.021$ & $<.002$ & .033 & $<.001$ \\
\hline ZK-58-19-805 & 303859097400901 & $03-30-00$ & $<.002$ & $<.09$ & $<.002$ & $<.34$ & $<.1$ & $<.021$ & $<.002$ & .032 & $<.001$ \\
\hline ZK-58-27-819 & 303117097421301 & 04-18-00 & $<.002$ & $<.09$ & $<.002$ & $<.47$ & $<.1$ & $<.021$ & $<.002$ & $<.001$ & $<.001$ \\
\hline ZP-46-16-102 & 315042103054301 & $01-12-00$ & $<.002$ & $<.09$ & $<.002$ & $<.37$ & $<.1$ & $<.021$ & $<.002$ & $<.001$ & $<.001$ \\
\hline ZR-19-42-612 & 331902097472301 & 04-04-00 & $<.002$ & $<.09$ & $<.002$ & $<.21$ & $<.1$ & $<.021$ & $<.002$ & $<.001$ & $<.001$ \\
\hline ZR-19-51-801 & 330906097411701 & 04-04-00 & $<.002$ & $<.09$ & $<.002$ & $<.26$ & $<.1$ & $<.021$ & $<.002$ & $<.001$ & $<.001$ \\
\hline ZR-19-51-805 & 330839097412401 & 04-04-00 & $<.002$ & $<.09$ & $<.002$ & $<.21$ & $<.1$ & $<.14$ & $<.002$ & $<.001$ & $<.001$ \\
\hline ZR-19-61-705 & 330014097285401 & $01-25-00$ & $<.002$ & $<.09$ & $<.002$ & $<.21$ & $<.1$ & $<.021$ & $<.002$ & $<.001$ & $<.001$ \\
\hline ZT-24-50-403 & 331051102511901 & $05-17-00$ & $<.002$ & $<.09$ & $<.002$ & $<.21$ & $<.1$ & $<.1$ & $<.002$ & $<.001$ & $<.001$ \\
\hline ZT-27-02-103 & 325819102501601 & $05-23-00$ & $<.002$ & $<.09$ & $<.002$ & $<.21$ & $<.1$ & $<.021$ & $<.002$ & $<.001$ & $<.001$ \\
\hline ZT-27-02-105 & 325819102503701 & $05-23-00$ & $<.002$ & $<.09$ & $<.002$ & $<.21$ & $<.1$ & $<.11$ & $<.002$ & .033 & $<.001$ \\
\hline
\end{tabular}


Table 5. Concentrations of soluble pesticides in public water-supply wells sampled in Texas, November 1999-January 2001-Continued

\begin{tabular}{|c|c|c|c|c|c|c|c|c|c|c|c|c|c|}
\hline Local identifier & $\begin{array}{l}\text { Station } \\
\text { number }\end{array}$ & Date & $\begin{array}{c}\text { Ben- } \\
\text { fluralin } \\
(\mu \mathrm{g} / \mathrm{L})\end{array}$ & $\begin{array}{l}\text { Ben- } \\
\text { tazon } \\
(\mu \mathrm{g} / \mathrm{L})\end{array}$ & $\begin{array}{c}\text { Bro- } \\
\text { macil } \\
(\mu \mathrm{g} / \mathrm{L})\end{array}$ & $\begin{array}{c}\text { Bromox- } \\
\text { ynil } \\
(\mu \mathrm{g} / \mathrm{L})\end{array}$ & $\begin{array}{l}\text { Buty- } \\
\text { late } \\
(\mu \mathrm{g} / \mathrm{L})\end{array}$ & $\begin{array}{l}\text { Carb- } \\
\text { aryl } \\
(\mu \mathrm{g} / \mathrm{L})\end{array}$ & $\begin{array}{l}\text { Carbo- } \\
\text { furan } \\
(\mu \mathrm{g} / \mathrm{L})\end{array}$ & $\begin{array}{l}\text { Chloro- } \\
\text { thalonil } \\
(\mu \mathrm{g} / \mathrm{L})\end{array}$ & $\begin{array}{l}\text { Chlor- } \\
\text { pyrifos } \\
(\mu \mathrm{g} / \mathrm{L})\end{array}$ & $\begin{array}{l}\text { Clopy- } \\
\text { ralid } \\
(\mu \mathrm{g} / \mathrm{L})\end{array}$ & $\begin{array}{l}\text { Cyan- } \\
\text { azine } \\
(\mu \mathrm{g} / \mathrm{L})\end{array}$ \\
\hline AB-27-37-103 & 322805102283801 & $05-17-00$ & $<0.002$ & $<0.035$ & $<0.06$ & $<0.04$ & $<0.002$ & $<0.07$ & $<0.29$ & $<0.48$ & $<0.004$ & $<0.23$ & $<0.004$ \\
\hline AB-27-37-105 & 322826102280101 & $05-17-00$ & $<.002$ & $<.035$ & $<.06$ & $<.04$ & $<.002$ & $<.07$ & $<.29$ & $<.48$ & $<.004$ & $<.23$ & $<.004$ \\
\hline $\mathrm{AK}-06-52-506$ & 351024101334401 & 05-04-00 & $<.002$ & $<.035$ & $<.27$ & $<.04$ & $<.002$ & $<.07$ & $<.29$ & $<.48$ & $<.004$ & $<.23$ & $<.004$ \\
\hline AP-59-62-403 & 300259096195001 & 04-03-00 & $<.002$ & $<.035$ & $<.06$ & $<.04$ & $<.002$ & $<.07$ & $<.29$ & $<.48$ & $<.004$ & $<.23$ & $<.004$ \\
\hline AP-66-16-407 & 294903096061401 & $04-21-00$ & $<.002$ & $<.035$ & $<.06$ & $<.04$ & $<.002$ & $<.07$ & $<.29$ & $<.48$ & $<.004$ & $<.23$ & $<.004$ \\
\hline AR-10-51-408 & 341229102435401 & $05-01-00$ & $<.002$ & $<.035$ & $<.06$ & $<.04$ & $<.002$ & $<.07$ & $<.29$ & $<.48$ & $<.004$ & $<.23$ & $<.004$ \\
\hline AR-24-09-604 & 335000102545701 & 05-09-00 & $<.002$ & $<.035$ & $<.06$ & $<.04$ & $<.002$ & $<.07$ & $<.29$ & $<.48$ & $<.004$ & $<.23$ & $<.004$ \\
\hline AT-58-62-206 & 300646097193001 & $12-10-99$ & $<.002$ & $<.035$ & $<.11$ & $<.04$ & $<.002$ & $<.07$ & $<.29$ & $<.48$ & $<.004$ & $<.23$ & $<.004$ \\
\hline AU-21-30-389 & 333612099163101 & $05-02-00$ & $<.002$ & $<.035$ & $<.06$ & $<.04$ & $<.002$ & $<.07$ & $<.29$ & $<.48$ & $<.004$ & $<.23$ & $<.004$ \\
\hline$A X-58-04-604$ & 305718097314401 & $03-22-00$ & $<.002$ & $<.035$ & $<.14$ & $<.04$ & $<.002$ & $<.07$ & $<.29$ & $<.48$ & $<.004$ & $<.23$ & $<.004$ \\
\hline AX-58-04-623 & 305624097321101 & $03-22-00$ & $<.002$ & $<.035$ & $<.06$ & $<.04$ & $<.002$ & $<.07$ & $<.29$ & $<.48$ & $<.004$ & $<.23$ & $<.004$ \\
\hline AY-68-35-105 & 292842098425001 & $03-06-00$ & $<.002$ & $<.035$ & $<.06$ & $<.04$ & $<.002$ & $<.07$ & $<.29$ & $<.48$ & $<.004$ & $<.23$ & $<.004$ \\
\hline AZ-57-45-612 & 301923098225401 & $04-18-00$ & $<.002$ & $<.035$ & $<.06$ & $<.04$ & $<.002$ & $<.07$ & $<.29$ & $<.48$ & $<.004$ & $<.23$ & $<.004$ \\
\hline AZ-57-45-811 & 301633098252901 & $03-29-00$ & $<.002$ & $<.035$ & $<.06$ & $<.04$ & $<.002$ & $<.07$ & $<.29$ & $<.48$ & $<.004$ & $<.23$ & $<.004$ \\
\hline BD-16-40-709 & 332301094061701 & $01-18-01$ & $<.01$ & $<.035$ & $<.09$ & $<.07$ & $<.002$ & $<.024$ & $<.29$ & $<.28$ & $<.005$ & $<.42$ & $<.018$ \\
\hline BK-73-47-504 & 291911103124301 & $12-28-00$ & $<.01$ & $<.035$ & $<.09$ & $<.07$ & $<.002$ & $<.024$ & $<.29$ & $<.28$ & $<.005$ & $<.42$ & $<.018$ \\
\hline BK-73-52-905 & 290819103312601 & $12-28-00$ & $<.01$ & $<.035$ & $<.09$ & $<.07$ & $<.002$ & $<.024$ & $<.29$ & $<.28$ & $<.005$ & $<.42$ & $<.018$ \\
\hline BL-11-38-334 & 342841101155301 & $05-01-00$ & $<.002$ & $<.035$ & $<.06$ & $<.04$ & $<.002$ & $<.07$ & $<.29$ & $<.48$ & $<.004$ & $<.23$ & $<.004$ \\
\hline BL-11-48-507 & 341919101035201 & $05-02-00$ & $<.002$ & $<.035$ & $<.06$ & $<.04$ & $<.002$ & $<.07$ & $<.29$ & $<.48$ & $<.004$ & $<.23$ & $<.004$ \\
\hline BL-12-41-204 & 342109100570301 & $05-02-00$ & $<.002$ & $<.035$ & $<.06$ & $<.04$ & $<.002$ & $<.07$ & $<.29$ & $<.48$ & $<.004$ & $<.23$ & $<.004$ \\
\hline BR-41-01-244 & 315849098552101 & $04-05-00$ & $<.002$ & $<.035$ & $<.06$ & $<.04$ & $<.002$ & $<.07$ & $<.29$ & $<.48$ & $<.004$ & $<.23$ & $<.004$ \\
\hline BT-57-14-403 & 304939098202401 & 03-08-00 & $<.002$ & $<.035$ & $<.06$ & $<.04$ & $<.002$ & $<.07$ & $<.29$ & $<.48$ & $<.004$ & $<.23$ & $<.004$ \\
\hline BT-57-14-404 & 304927098211201 & 03-08-00 & $<.002$ & $<.035$ & $<.06$ & $<.04$ & $<.002$ & $<.07$ & $<.29$ & $<.48$ & $<.004$ & $<.23$ & $<.004$ \\
\hline BT-57-21-607 & 304125098224001 & $03-28-00$ & $<.002$ & $<.035$ & $<.06$ & $<.04$ & $<.002$ & $<.07$ & $<.29$ & $<.48$ & $<.004$ & $<.23$ & $<.004$ \\
\hline BT-57-21-608 & 304121098224201 & $03-28-00$ & $<.002$ & $<.035$ & $<.06$ & $<.04$ & $<.002$ & $<.07$ & $<.29$ & $<.48$ & $<.004$ & $<.23$ & $<.004$ \\
\hline BT-57-23-110 & 304406098130801 & 03-29-00 & $<.002$ & $<.035$ & $<.06$ & $<.04$ & $<.002$ & $<.07$ & $<.29$ & $<.48$ & $<.004$ & $<.23$ & $<.004$ \\
\hline BT-57-30-101 & 303548098211701 & 03-01-00 & $<.002$ & $<.035$ & $<.06$ & $<.04$ & $<.002$ & $<.07$ & $<.29$ & $<.48$ & $<.004$ & $<.23$ & $<.004$ \\
\hline BU-67-10-910 & 294528097464401 & $03-21-00$ & $<.002$ & $<.035$ & $<.4$ & $<.04$ & $<.002$ & $<.07$ & $<.29$ & $<.48$ & $<.004$ & $<.23$ & $<.004$ \\
\hline
\end{tabular}


Table 5. Concentrations of soluble pesticides in public water-supply wells sampled in Texas, November 1999-January 2001-Continued

\begin{tabular}{|c|c|c|c|c|c|c|c|c|c|c|c|c|c|}
\hline Local identifier & $\begin{array}{l}\text { Station } \\
\text { number }\end{array}$ & Date & $\begin{array}{l}\text { Ben- } \\
\text { fluralin } \\
(\mu \mathrm{g} / \mathrm{L})\end{array}$ & $\begin{array}{l}\text { Ben- } \\
\text { tazon } \\
(\mu \mathrm{g} / \mathrm{L})\end{array}$ & $\begin{array}{l}\text { Bro- } \\
\text { macil } \\
(\mu \mathrm{g} / \mathrm{L})\end{array}$ & $\begin{array}{c}\text { Bromox- } \\
\text { ynil } \\
(\mu \mathrm{g} / \mathrm{L})\end{array}$ & $\begin{array}{l}\text { Buty- } \\
\text { late } \\
(\mu g / L)\end{array}$ & $\begin{array}{l}\text { Carb- } \\
\text { aryl } \\
(\mu \mathrm{g} / \mathrm{L})\end{array}$ & $\begin{array}{l}\text { Carbo- } \\
\text { furan } \\
(\mu \mathrm{g} / \mathrm{L})\end{array}$ & $\begin{array}{c}\text { Chloro- } \\
\text { thalonil } \\
(\mu \mathrm{g} / \mathrm{L})\end{array}$ & $\begin{array}{l}\text { Chlor- } \\
\text { pyrifos } \\
(\mu \mathrm{g} / L)\end{array}$ & $\begin{array}{l}\text { Clopy- } \\
\text { ralid } \\
(\mu \mathrm{g} / \mathrm{L})\end{array}$ & $\begin{array}{l}\text { Cyan- } \\
\text { azine } \\
(\mu g / L)\end{array}$ \\
\hline BX-30-55-936 & 320939099093801 & $12-18-00$ & $<0.01$ & $<0.035$ & $<0.09$ & $<0.07$ & $<0.002$ & $<0.024$ & $<0.29$ & $<0.13$ & $<0.005$ & $<0.42$ & $<0.018$ \\
\hline DL-14-62-604 & 340406098160701 & $05-10-00$ & $<.002$ & $<.035$ & $<.06$ & $<.04$ & $<.002$ & $<.07$ & $<.29$ & $<.48$ & $<.004$ & $<.23$ & $<.004$ \\
\hline DP-24-18-308 & 334352102450601 & $05-09-00$ & $<.002$ & $<.035$ & $<.06$ & $<.04$ & $<.002$ & $<.07$ & $<.29$ & $<.48$ & $<.004$ & $<.23$ & $<.004$ \\
\hline DP-25-24-304 & 334245103011001 & $05-08-00$ & $<.002$ & $<.035$ & $<.06$ & $<.04$ & $<.002$ & $<.07$ & $<.29$ & $<.48$ & $<.004$ & $<.23$ & $<.004$ \\
\hline DU-12-06-609 & 345658100155501 & $05-08-00$ & $<.002$ & $<.035$ & $<.06$ & $<.04$ & $<.002$ & $<.07$ & $<.29$ & $<.48$ & $<.004$ & $<.23$ & $<.004$ \\
\hline DX-68-05-621 & 295520098242301 & $03-22-00$ & $<.002$ & $<.035$ & $<.06$ & $<.04$ & $<.002$ & $<.07$ & $<.29$ & $<.48$ & $<.004$ & $<.23$ & $<.004$ \\
\hline DX-68-06-405 & 295528098222301 & $03-22-00$ & $<.002$ & $<.035$ & $<.06$ & $<.04$ & $<.002$ & $<.07$ & $<.29$ & $<.48$ & $<.004$ & $<.23$ & $<.004$ \\
\hline DY-41-13-307 & 315105098241401 & $04-05-00$ & $<.002$ & .049 & $<.06$ & $<.04$ & $<.002$ & $<.07$ & $<.29$ & $<.48$ & $<.004$ & $<.23$ & $<.004$ \\
\hline HH-45-35-505 & 312715102402201 & $01-12-00$ & $<.002$ & $<.035$ & $<.1$ & $<.04$ & $<.002$ & $<.07$ & $<.29$ & $<.48$ & $<.004$ & $<.23$ & $<.004$ \\
\hline HP-12-62-802 & 340210100184901 & $05-05-00$ & $<.002$ & $<.035$ & -- & $<.04$ & $<.002$ & -- & -- & -- & $<.004$ & $<.23$ & $<.004$ \\
\hline HS-28-09-404 & 324810101591201 & $05-16-00$ & $<.002$ & $<.035$ & .058 & $<.04$ & $<.002$ & $<.07$ & $<.29$ & $<.48$ & $<.004$ & $<.23$ & $<.004$ \\
\hline HT-07-63-204 & 350607102103401 & $05-03-00$ & $<.002$ & $<.035$ & $<.06$ & $<.04$ & $<.002$ & $<.07$ & $<.29$ & $<.48$ & $<.004$ & $<.23$ & $<.004$ \\
\hline HZ-77-34-606 & 282608099455301 & $03-28-00$ & $<.002$ & $<.035$ & $<.06$ & $<.04$ & $<.002$ & $<.07$ & $<.29$ & $<.48$ & $<.004$ & $<.23$ & $<.004$ \\
\hline JA-05-57-613 & 350254100535501 & $05-04-00$ & $<.002$ & $<.035$ & $<.06$ & $<.04$ & $<.002$ & $<.07$ & $<.29$ & $<.48$ & $<.004$ & $<.23$ & $<.004$ \\
\hline JA-12-12-404 & 344910100360401 & 05-02-00 & $<.002$ & $<.035$ & $<.06$ & $<.04$ & $<.002$ & $<.07$ & $<.29$ & $<.48$ & $<.004$ & $<.23$ & $<.004$ \\
\hline $\mathrm{JH}-45-05-924$ & 315245102240201 & $05-25-00$ & $<.002$ & $<.035$ & .214 & $<.04$ & $<.002$ & $<.07$ & $<.29$ & $<.48$ & $<.004$ & $<.23$ & $<.004$ \\
\hline JL-49-04-116 & 315757106370201 & $01-06-00$ & $<.002$ & $<.035$ & $<.13$ & $<.04$ & $<.002$ & $<.07$ & $<.29$ & $<.48$ & $<.004$ & $<.23$ & $<.004$ \\
\hline JL-49-04-423 & 315708106362301 & $01-03-00$ & $<.002$ & $<.035$ & $<.14$ & $<.04$ & $<.002$ & $<.07$ & $<.29$ & $<.48$ & $<.004$ & $<.23$ & $<.004$ \\
\hline JL-49-04-428 & 315517106361401 & $01-06-00$ & $<.002$ & $<.035$ & $<.12$ & $<.04$ & $<.002$ & $<.07$ & $<.29$ & $<.48$ & $<.004$ & $<.23$ & $<.004$ \\
\hline JL-49-04-492 & 315623106360601 & $01-06-00$ & $<.002$ & $<.035$ & $<.13$ & $<.04$ & $<.002$ & $<.07$ & $<.29$ & $<.48$ & $<.004$ & $<.23$ & $<.004$ \\
\hline JL-49-22-626 & 314145106163601 & 01-04-00 & $<.002$ & $<.035$ & $<.11$ & $<.04$ & $<.002$ & $<.07$ & $<.29$ & $<.48$ & $<.004$ & $<.23$ & $<.004$ \\
\hline JL-49-24-423 & 314107106063301 & 01-07-00 & $<.002$ & $<.035$ & $<.12$ & $<.04$ & $<.002$ & $<.07$ & $<.29$ & $<.48$ & $<.004$ & $<.23$ & $<.004$ \\
\hline JL-49-40-502 & 312648106044701 & $01-04-00$ & $<.002$ & $<.035$ & $<.11$ & $<.04$ & $<.002$ & $<.07$ & $<.29$ & $<.48$ & $<.004$ & $<.23$ & $<.004$ \\
\hline JY-65-26-520 & 293314095474702 & 04-03-00 & $<.002$ & $<.035$ & $<.06$ & $<.04$ & $<.002$ & $<.07$ & $<.29$ & $<.48$ & $<.004$ & $<.23$ & $<.004$ \\
\hline KD-27-01-612 & 325523102542701 & $05-22-00$ & $<.002$ & $<.035$ & $<.06$ & $<.04$ & $<.002$ & $<.07$ & $<.29$ & $<.48$ & $<.004$ & $<.23$ & $<.004$ \\
\hline KK-57-42-709 & 301545098502801 & $07-06-00$ & $<.002$ & $<.035$ & $<.06$ & $<.04$ & $<.002$ & $<.003$ & $<.003$ & $<.48$ & $<.004$ & $<.23$ & $<.004$ \\
\hline KK-57-50-325 & 301357098471901 & $03-28-00$ & $<.002$ & $<.035$ & $<.06$ & $<.04$ & $<.002$ & $<.07$ & $<.29$ & $<.48$ & $<.004$ & $<.23$ & $<.004$ \\
\hline KK-57-51-303 & 301411098383101 & $03-21-00$ & $<.002$ & $<.035$ & $<.06$ & $<.04$ & $<.002$ & $<.07$ & $<.29$ & $<.48$ & $<.004$ & $<.23$ & $<.004$ \\
\hline KK-57-52-107 & 301430098363401 & $03-21-00$ & $<.002$ & $<.035$ & $<.06$ & $<.04$ & $<.002$ & $<.07$ & $<.29$ & $<.48$ & $<.004$ & $<.23$ & $<.004$ \\
\hline
\end{tabular}


Table 5. Concentrations of soluble pesticides in public water-supply wells sampled in Texas, November 1999-January 2001-Continued

\begin{tabular}{|c|c|c|c|c|c|c|c|c|c|c|c|c|c|}
\hline Local identifier & $\begin{array}{l}\text { Station } \\
\text { number }\end{array}$ & Date & $\begin{array}{c}\text { Ben- } \\
\text { fluralin } \\
(\mu \mathrm{g} / \mathrm{L})\end{array}$ & $\begin{array}{l}\text { Ben- } \\
\text { tazon } \\
(\mu \mathrm{g} / \mathrm{L})\end{array}$ & $\begin{array}{l}\text { Bro- } \\
\text { macil } \\
(\mu \mathrm{g} / \mathrm{L})\end{array}$ & $\begin{array}{c}\text { Bromox- } \\
\text { ynil } \\
(\mu \mathrm{g} / \mathrm{L})\end{array}$ & $\begin{array}{l}\text { Buty- } \\
\text { late } \\
(\mu \mathrm{g} / \mathrm{L})\end{array}$ & $\begin{array}{c}\text { Carb- } \\
\text { aryl } \\
(\mu \mathrm{g} / \mathrm{L})\end{array}$ & $\begin{array}{l}\text { Carbo- } \\
\text { furan } \\
(\mu \mathrm{g} / \mathrm{L})\end{array}$ & $\begin{array}{c}\text { Chloro- } \\
\text { thalonil } \\
(\mu \mathrm{g} / \mathrm{L})\end{array}$ & $\begin{array}{c}\text { Chlor- } \\
\text { pyrifos } \\
(\mu \mathrm{g} / \mathrm{L})\end{array}$ & $\begin{array}{l}\text { Clopy- } \\
\text { ralid } \\
(\mu \mathrm{g} / L)\end{array}$ & $\begin{array}{l}\text { Cyan- } \\
\text { azine } \\
(\mu \mathrm{g} / \mathrm{L})\end{array}$ \\
\hline KP-79-23-101 & 284256097125001 & $02-29-00$ & $<0.002$ & $<0.035$ & $<0.06$ & $<0.04$ & $<0.002$ & $<0.07$ & $<0.29$ & $<0.48$ & $<0.004$ & $<0.23$ & $<0.004$ \\
\hline KS-05-52-105 & 351428100352601 & $05-04-00$ & $<.002$ & $<.035$ & $<.06$ & $<.04$ & $<.002$ & $<.07$ & $<.29$ & $<.48$ & $<.004$ & $<.23$ & $<.004$ \\
\hline KT-18-11-405 & 334844096435701 & 04-19-00 & $<.002$ & $<.035$ & $<.06$ & $<.04$ & $<.002$ & $<.07$ & $<.29$ & $<.48$ & $<.004$ & $<.23$ & $<.004$ \\
\hline KT-18-11-406 & 334840096434001 & 04-19-00 & $<.002$ & $<.035$ & $<.06$ & $<.04$ & $<.002$ & $<.07$ & $<.29$ & $<.48$ & $<.004$ & $<.23$ & $<.004$ \\
\hline KT-18-28-703 & 333039096362901 & 04-19-00 & $<.002$ & $<.035$ & $<.06$ & $<.04$ & $<.002$ & $<.07$ & $<.29$ & $<.48$ & $<.004$ & $<.23$ & $<.004$ \\
\hline KY-11-49-512 & 341116101574601 & $05-04-00$ & $<.002$ & $<.035$ & $<.06$ & $<.04$ & $<.002$ & $<.07$ & $<.29$ & $<.48$ & $<.004$ & $<.23$ & $<.004$ \\
\hline KY-11-51-416 & 341032101423801 & $05-04-00$ & $<.002$ & $<.035$ & $<.06$ & $<.04$ & $<.002$ & $<.07$ & $<.29$ & $<.48$ & $<.004$ & $<.23$ & $<.004$ \\
\hline KY-11-57-602 & 340345101531801 & $05-03-00$ & $<.002$ & $<.035$ & $<.06$ & $<.04$ & $<.002$ & $<.07$ & $<.29$ & $<.48$ & $<.004$ & $<.23$ & $<.004$ \\
\hline KZ-12-19-104 & 344234100431601 & 05-09-00 & $<.002$ & $<.035$ & $<.06$ & $<.04$ & $<.002$ & $<.07$ & $<.29$ & $<.48$ & $<.004$ & $<.23$ & $<.004$ \\
\hline LD-13-44-907 & 341523099305701 & $12-19-00$ & $<.01$ & $<.035$ & $<.09$ & $<.07$ & $<.002$ & $<.024$ & $<.29$ & $<.13$ & $<.005$ & $<.42$ & $<.018$ \\
\hline LJ-60-64-807 & 300149095045001 & $05-04-00$ & $<.002$ & $<.035$ & $<.06$ & $<.04$ & $<.002$ & $<.07$ & $<.29$ & $<.48$ & $<.004$ & $<.23$ & $<.004$ \\
\hline LP-21-49-317 & 331233099545201 & $05-01-00$ & $<.002$ & $<.035$ & $<.06$ & $<.04$ & $<.002$ & $<.07$ & $<.29$ & $<.48$ & $<.004$ & $<.23$ & $<.004$ \\
\hline LR-67-09-112 & 295137097585801 & $03-21-00$ & $<.002$ & $<.035$ & $<.06$ & $<.04$ & $<.002$ & $<.07$ & $<.29$ & $<.48$ & $<.004$ & $<.23$ & $<.004$ \\
\hline LX-24-15-612 & 334857102092301 & $05-11-00$ & $<.002$ & $<.035$ & $<.06$ & $<.04$ & $<.002$ & $<.07$ & $<.29$ & $<.48$ & $<.004$ & $<.23$ & $<.004$ \\
\hline LX-24-29-306 & 333536102223101 & 05-09-00 & $<.002$ & $<.035$ & $<.06$ & $<.04$ & $<.002$ & $<.07$ & $<.29$ & $<.48$ & $<.004$ & $<.23$ & $<.004$ \\
\hline LX-24-37-405 & 332714102285401 & $05-16-00$ & $<.002$ & $<.035$ & .135 & $<.04$ & $<.002$ & $<.07$ & $<.29$ & $<.48$ & $<.004$ & $<.23$ & $<.004$ \\
\hline LX-24-40-405 & 332624102072901 & $05-17-00$ & $<.002$ & $<.035$ & $<.06$ & $<.04$ & $<.002$ & $<.07$ & $<.29$ & $<.48$ & $<.004$ & $<.23$ & $<.004$ \\
\hline LY-32-26-706 & 323204097500301 & $03-22-00$ & $<.002$ & $<.035$ & $<.06$ & $<.04$ & $<.002$ & $<.07$ & $<.29$ & $<.48$ & $<.004$ & $<.23$ & $<.004$ \\
\hline LY-32-34-208 & 322958097481301 & $03-21-00$ & $<.002$ & $<.035$ & $<.06$ & $<.04$ & $<.002$ & $<.07$ & $<.29$ & $<.48$ & $<.004$ & $<.23$ & $<.004$ \\
\hline LY-32-34-303 & 322928097461701 & 04-03-00 & $<.002$ & $<.035$ & $<.06$ & $<.04$ & $<.002$ & $<.07$ & $<.29$ & $<.48$ & $<.004$ & $<.23$ & $<.004$ \\
\hline LY-32-34-510 & 322537097485701 & $03-21-00$ & $<.002$ & $<.035$ & $<.06$ & $<.04$ & $<.002$ & $<.07$ & $<.29$ & $<.48$ & $<.004$ & $<.23$ & $<.004$ \\
\hline LY-32-34-609 & 322626097454801 & $03-21-00$ & $<.002$ & $<.035$ & $<.06$ & $<.04$ & $<.002$ & $<.07$ & $<.29$ & $<.48$ & $<.004$ & $<.23$ & $<.004$ \\
\hline PB-28-62-105 & 320649101215501 & $04-26-00$ & $<.002$ & $<.035$ & $<.06$ & $<.04$ & $<.002$ & $<.07$ & $<.29$ & $<.48$ & $<.004$ & $<.23$ & $<.004$ \\
\hline PK-43-50-216 & 311422100490201 & $12-20-00$ & $<.01$ & $<.035$ & $<.09$ & $<.07$ & $<.002$ & $<.024$ & $<.29$ & $<.13$ & $<.005$ & $<.42$ & $<.018$ \\
\hline PS-52-02-404 & 305502103504101 & 01-10-00 & $<.002$ & $<.035$ & $<.14$ & $<.04$ & $<.002$ & $<.07$ & $<.29$ & $<.48$ & $<.004$ & $<.23$ & $<.004$ \\
\hline PU-84-43-504 & 271841098402801 & 04-04-00 & $<.002$ & $<.035$ & $<.06$ & $<.04$ & $<.002$ & $<.07$ & $<.29$ & $<.48$ & $<.004$ & $<.23$ & $<.004$ \\
\hline$P X-32-47-109$ & 322201097141601 & $03-20-00$ & $<.002$ & $<.035$ & $<.06$ & $<.04$ & $<.002$ & $<.07$ & $<.29$ & $<.48$ & $<.004$ & $<.23$ & $<.004$ \\
\hline PZ-79-10-807 & 284713097493901 & $02-23-00$ & $<.002$ & $<.035$ & $<.06$ & $<.04$ & $<.002$ & $<.07$ & $<.29$ & $<.48$ & $<.004$ & $<.23$ & $<.004$ \\
\hline RS-21-35-103 & 332854099441101 & $05-01-00$ & $<.002$ & $<.035$ & $<.06$ & $<.04$ & $<.002$ & $<.07$ & $<.29$ & $<.48$ & $<.004$ & $<.23$ & $<.004$ \\
\hline RU-10-53-312 & 341418102243401 & $05-02-00$ & $<.002$ & $<.035$ & $<.06$ & $<.04$ & $<.002$ & $<.07$ & $<.29$ & $<.48$ & $<.004$ & $<.23$ & $<.004$ \\
\hline
\end{tabular}


Table 5. Concentrations of soluble pesticides in public water-supply wells sampled in Texas, November 1999-January 2001-Continued

\begin{tabular}{|c|c|c|c|c|c|c|c|c|c|c|c|c|c|}
\hline Local identifier & $\begin{array}{l}\text { Station } \\
\text { number }\end{array}$ & Date & $\begin{array}{l}\text { Ben- } \\
\text { fluralin } \\
(\mu \mathrm{g} / \mathrm{L})\end{array}$ & $\begin{array}{l}\text { Ben- } \\
\text { tazon } \\
(\mu \mathrm{g} / \mathrm{L})\end{array}$ & $\begin{array}{l}\text { Bro- } \\
\text { macil } \\
(\mu \mathrm{g} / \mathrm{L})\end{array}$ & $\begin{array}{c}\text { Bromox- } \\
\text { ynil } \\
(\mu \mathrm{g} / \mathrm{L})\end{array}$ & $\begin{array}{l}\text { Buty- } \\
\text { late } \\
(\mu g / L)\end{array}$ & $\begin{array}{l}\text { Carb- } \\
\text { aryl } \\
(\mu \mathrm{g} / \mathrm{L})\end{array}$ & $\begin{array}{l}\text { Carbo- } \\
\text { furan } \\
(\mu \mathrm{g} / \mathrm{L})\end{array}$ & $\begin{array}{c}\text { Chloro- } \\
\text { thalonil } \\
(\mu \mathrm{g} / \mathrm{L})\end{array}$ & $\begin{array}{l}\text { Chlor- } \\
\text { pyrifos } \\
(\mu \mathrm{g} / L)\end{array}$ & $\begin{array}{l}\text { Clopy- } \\
\text { ralid } \\
(\mu \mathrm{g} / \mathrm{L})\end{array}$ & $\begin{array}{l}\text { Cyan- } \\
\text { azine } \\
(\mu g / L)\end{array}$ \\
\hline RU-10-60-606 & 340427102302801 & $05-02-00$ & $<0.002$ & $<0.035$ & $<0.06$ & $<0.04$ & $<0.002$ & $<0.07$ & $<0.29$ & $<0.48$ & $<0.004$ & $<0.23$ & $<0.004$ \\
\hline SK-57-19-201 & 304323098421701 & $03-08-00$ & $<.002$ & $<.035$ & $<.06$ & $<.04$ & $<.002$ & $<.07$ & $<.29$ & $<.48$ & $<.004$ & $<.23$ & $<.004$ \\
\hline SK-57-34-201 & 303001098485201 & $03-09-00$ & $<.002$ & $<.035$ & $<.06$ & $<.04$ & $<.002$ & $<.07$ & $<.29$ & $<.48$ & $<.004$ & $<.23$ & $<.004$ \\
\hline SP-23-17-417 & 334043101585701 & $05-10-00$ & $<.002$ & $<.035$ & $<.06$ & $<.04$ & $<.002$ & $<.07$ & $<.29$ & $<.48$ & $<.004$ & $<.23$ & $<.004$ \\
\hline SP-23-18-107 & 334306101504101 & $05-10-00$ & $<.002$ & $<.035$ & $<.06$ & $<.04$ & $<.002$ & $<.07$ & $<.29$ & $<.48$ & $<.004$ & $<.23$ & $<.004$ \\
\hline SP-23-19-812 & 333955101404601 & 05-10-00 & $<.002$ & $<.035$ & $<.06$ & $<.04$ & $<.002$ & $<.07$ & $<.29$ & $<.48$ & $<.004$ & $<.23$ & $<.004$ \\
\hline SP-24-24-214 & 334419102045201 & $05-18-00$ & $<.002$ & $<.035$ & $<.06$ & $<.04$ & $<.002$ & $<.07$ & $<.29$ & $<.48$ & $<.004$ & $<.23$ & $<.004$ \\
\hline SR-23-41-602 & 331933101543701 & $05-15-00$ & $<.002$ & $<.035$ & $<.06$ & $<.04$ & $<.002$ & $<.07$ & $<.29$ & $<.48$ & $<.004$ & $<.23$ & $<.004$ \\
\hline SS-42-52-504 & 311202099321401 & $03-01-00$ & $<.002$ & $<.035$ & $<.06$ & $<.04$ & $<.002$ & $<.07$ & $<.29$ & $<.48$ & $<.004$ & $<.23$ & $<.004$ \\
\hline ST-40-39-304 & 312745097083301 & $03-23-00$ & $<.002$ & $<.035$ & $<.06$ & $<.04$ & $<.002$ & $<.07$ & $<.29$ & $<.48$ & $<.004$ & $<.23$ & $<.004$ \\
\hline SY-27-39-505 & 322555102105501 & $05-24-00$ & $<.002$ & $<.035$ & $<.06$ & $<.04$ & $<.002$ & $<.07$ & $<.29$ & $<.48$ & $<.004$ & $<.23$ & $<.004$ \\
\hline SY-28-50-908 & 320816101474601 & $05-23-00$ & $<.002$ & $<.035$ & $<.82$ & $<.04$ & $<.002$ & $<.07$ & $<.43$ & $<.48$ & $<.004$ & $<.23$ & $<.004$ \\
\hline TB-76-03-605 & 285657100373801 & $03-28-00$ & $<.002$ & $<.035$ & $<.06$ & $<.04$ & $<.002$ & $<.07$ & $<.29$ & $<.48$ & $<.004$ & $<.23$ & $<.004$ \\
\hline TJ-27-63-712 & 320143102123101 & $12-20-00$ & $<.01$ & $<.035$ & $<.09$ & $<.07$ & $<.002$ & $<.024$ & $<.29$ & $<.13$ & $<.005$ & $<.42$ & $<.018$ \\
\hline TJ-28-57-702 & 320221101594901 & $05-25-00$ & $<.002$ & $<.035$ & $<.06$ & $<.04$ & $<.002$ & $<.07$ & $<.29$ & $<.48$ & $<.004$ & $<.23$ & $<.004$ \\
\hline TJ-28-57-903 & 320010101523701 & $05-23-00$ & $<.002$ & $<.035$ & $<.06$ & $<.04$ & $<.002$ & $<.07$ & $<.29$ & $<.48$ & $<.004$ & $<.23$ & $<.004$ \\
\hline TJ-45-07-407 & 315643102131101 & $05-24-00$ & $<.002$ & $<.035$ & $<.06$ & $<.04$ & $<.002$ & $<.07$ & $<.29$ & $<.48$ & $<.004$ & $<.23$ & $<.004$ \\
\hline TJ-45-07-606 & 315702102075401 & $05-25-00$ & $<.002$ & $<.035$ & $<.11$ & $<.04$ & $<.002$ & $<.07$ & $<.29$ & $<.48$ & $<.004$ & $<.23$ & $<.004$ \\
\hline TS-60-36-510 & 302701095331201 & 04-20-00 & $<.002$ & $<.035$ & $<.06$ & $<.04$ & $<.002$ & $<.07$ & $<.29$ & $<.48$ & $<.004$ & $<.23$ & $<.004$ \\
\hline TW-22-02-714 & 335416100502101 & $05-05-00$ & $<.002$ & $<.035$ & $<.06$ & $<.04$ & $<.002$ & $<.07$ & $<.29$ & $<.48$ & $<.004$ & $<.23$ & $<.004$ \\
\hline TW-22-02-716 & 335449100513101 & $12-19-00$ & $<.01$ & $<.035$ & $<.09$ & $<.07$ & $<.002$ & $<.024$ & $<.29$ & $<.13$ & $<.005$ & $<.42$ & $<.018$ \\
\hline UJ-62-49-712 & 300906093585201 & 04-28-00 & $<.002$ & $<.035$ & $<.06$ & $<.04$ & $<.002$ & $<.07$ & $<.29$ & $<.48$ & $<.004$ & $<.23$ & $<.004$ \\
\hline UK-31-05-501 & 325545098255501 & $03-22-00$ & $<.002$ & $<.035$ & $<.06$ & $<.04$ & $<.002$ & $<.07$ & $<.29$ & $<.48$ & $<.004$ & $<.23$ & $<.004$ \\
\hline $\mathrm{UK}-31-24-501$ & 324210098034701 & $03-22-00$ & $<.002$ & $<.035$ & $<.06$ & $<.04$ & $<.002$ & $<.07$ & $<.29$ & $<.48$ & $<.004$ & $<.23$ & $<.004$ \\
\hline UL-35-48-202 & 322207094034501 & $04-25-00$ & $<.002$ & $<.035$ & $<.06$ & $<.04$ & $<.002$ & $<.07$ & $<.29$ & $<.48$ & $<.004$ & $<.23$ & $<.004$ \\
\hline UP-32-10-604 & 324926097455001 & 02-09-00 & $<.002$ & $<.035$ & $<.16$ & $<.04$ & $<.002$ & $<.07$ & $<.29$ & $<.48$ & $<.004$ & $<.23$ & $<.004$ \\
\hline UP-32-11-103 & 325005097440101 & 02-09-00 & $<.002$ & $<.035$ & $<.13$ & $<.04$ & $<.002$ & $<.07$ & $<.29$ & $<.48$ & $<.004$ & $<.23$ & $<.004$ \\
\hline UP-32-11-709 & 324649097442001 & 02-09-00 & $<.002$ & $<.035$ & $<.13$ & $<.04$ & $<.002$ & $<.07$ & $<.29$ & $<.48$ & $<.004$ & $<.23$ & $<.004$ \\
\hline
\end{tabular}


Table 5. Concentrations of soluble pesticides in public water-supply wells sampled in Texas, November 1999-January 2001-Continued

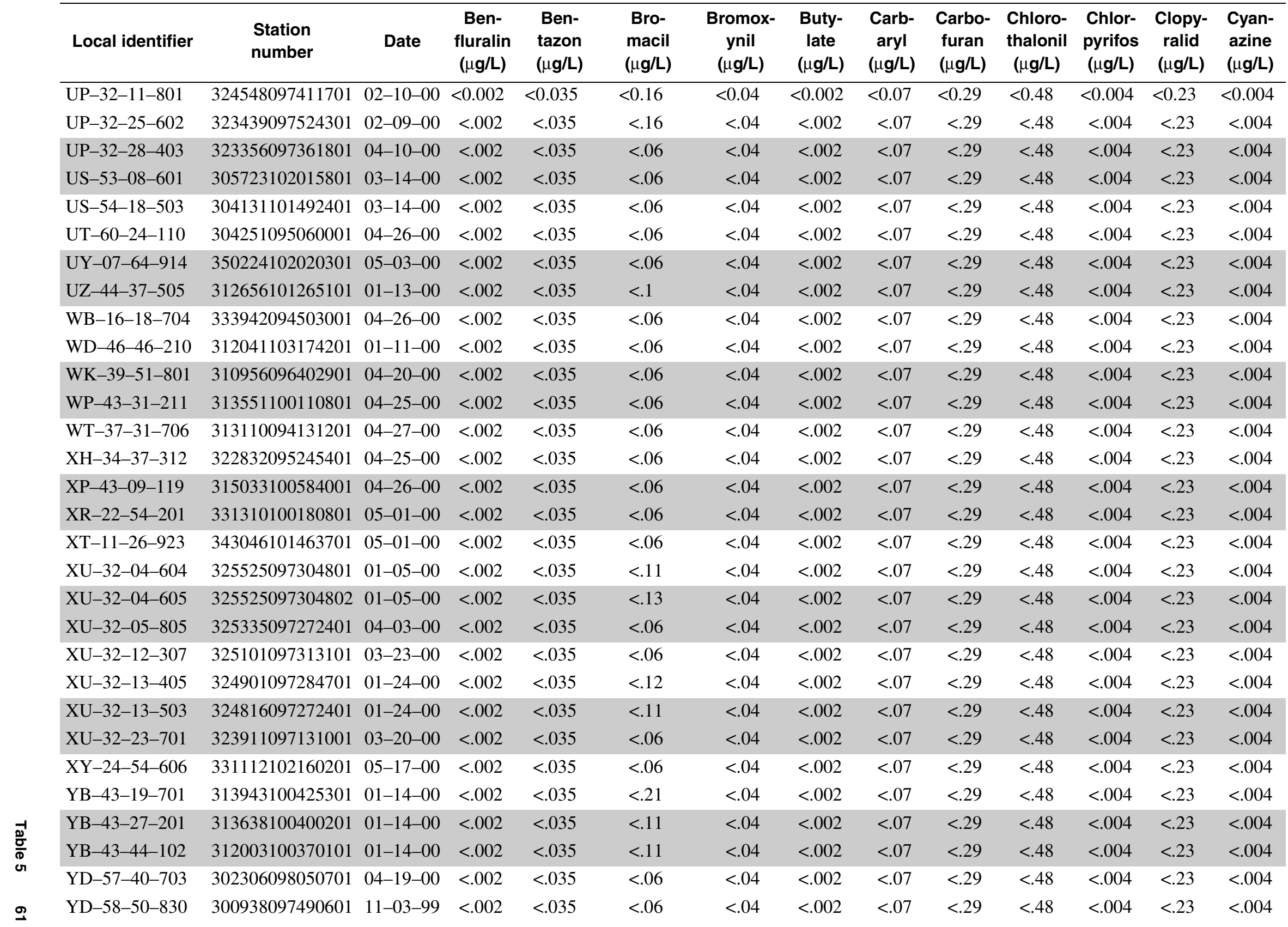


Table 5. Concentrations of soluble pesticides in public water-supply wells sampled in Texas, November 1999-January 2001-Continued

\begin{tabular}{|c|c|c|c|c|c|c|c|c|c|c|c|c|c|}
\hline Local identifier & $\begin{array}{l}\text { Station } \\
\text { number }\end{array}$ & Date & $\begin{array}{l}\text { Ben- } \\
\text { fluralin } \\
(\mu \mathrm{g} / \mathrm{L})\end{array}$ & $\begin{array}{l}\text { Ben- } \\
\text { tazon } \\
(\mu \mathrm{g} / \mathrm{L})\end{array}$ & $\begin{array}{l}\text { Bro- } \\
\text { macil } \\
(\mu \mathrm{g} / \mathrm{L})\end{array}$ & $\begin{array}{c}\text { Bromox- } \\
\text { ynil } \\
(\mu \mathrm{g} / \mathrm{L})\end{array}$ & $\begin{array}{l}\text { Buty- } \\
\text { late } \\
(\mu \mathrm{g} / \mathrm{L})\end{array}$ & $\begin{array}{l}\text { Carb- } \\
\text { aryl } \\
(\mu \mathrm{g} / \mathrm{L})\end{array}$ & $\begin{array}{l}\text { Carbo- } \\
\text { furan } \\
(\mu \mathrm{g} / \mathrm{L})\end{array}$ & $\begin{array}{l}\text { Chloro- } \\
\text { thalonil } \\
(\mu \mathrm{g} / \mathrm{L})\end{array}$ & $\begin{array}{l}\text { Chlor- } \\
\text { pyrifos } \\
(\mu \mathrm{g} / \mathrm{L})\end{array}$ & $\begin{array}{l}\text { Clopy- } \\
\text { ralid } \\
(\mu \mathrm{g} / \mathrm{L})\end{array}$ & $\begin{array}{l}\text { Cyan- } \\
\text { azine } \\
(\mu \mathrm{g} / \mathrm{L})\end{array}$ \\
\hline YD-58-52-304 & 301305097314601 & $04-27-00$ & $<0.002$ & $<0.035$ & $<0.06$ & $<0.04$ & $<0.002$ & $<0.07$ & $<0.14$ & $<0.48$ & $<0.004$ & $<0.23$ & $<0.004$ \\
\hline YP-69-27-107 & 293652099442501 & $10-19-00$ & $<.01$ & $<.035$ & $<.09$ & $<.07$ & $<.002$ & $<.024$ & $<.29$ & $<.28$ & $<.005$ & $<.42$ & $<.018$ \\
\hline YP-69-27-402 & 293450099433701 & $10-19-00$ & $<.01$ & $<.035$ & $<.09$ & $<.07$ & $<.002$ & $<.024$ & $<.29$ & $<.28$ & $<.005$ & $<.42$ & $<.018$ \\
\hline YP-69-27-704 & 293037099430301 & $10-18-00$ & $<.01$ & $<.035$ & $<.09$ & $<.07$ & $<.002$ & $<.024$ & $<.29$ & $<.28$ & $<.005$ & $<.42$ & $<.018$ \\
\hline YX-46-32-625 & 313252103010301 & $01-11-00$ & $<.002$ & $<.035$ & $<.13$ & $<.04$ & $<.002$ & $<.07$ & $<.29$ & $<.48$ & $<.004$ & $<.23$ & $<.004$ \\
\hline YX-46-40-311 & 312848103012901 & $01-11-00$ & $<.002$ & $<.035$ & $<.21$ & $<.04$ & $<.002$ & $<.07$ & $<.29$ & $<.48$ & $<.004$ & $<.23$ & $<.004$ \\
\hline YY-59-54-902 & 300824096152400 & 04-19-00 & $<.002$ & $<.035$ & $<.06$ & $<.04$ & $<.002$ & $<.07$ & $<.29$ & & $<.004$ & $<.23$ & $<.004$ \\
\hline ZB-05-29-819 & 353215100261201 & $05-10-00$ & $<.002$ & $<.035$ & $<.06$ & $<.04$ & $<.002$ & $<.07$ & $<.29$ & $<.48$ & $<.004$ & $<.23$ & $<.004$ \\
\hline ZB-05-38-512 & 352709100182901 & $05-08-00$ & $<.002$ & $<.035$ & $<.06$ & $<.04$ & $<.002$ & $<.07$ & $<.29$ & $<.48$ & $<.004$ & $<.23$ & $<.004$ \\
\hline ZK-58-19-805 & 303859097400901 & $03-30-00$ & $<.002$ & $<.035$ & $<.06$ & $<.04$ & $<.002$ & $<.07$ & $<.29$ & $<.48$ & $<.004$ & $<.23$ & $<.004$ \\
\hline ZK-58-27-819 & 303117097421301 & $04-18-00$ & $<.002$ & $<.035$ & $<.06$ & $<.04$ & $<.002$ & $<.07$ & $<.29$ & $<.48$ & $<.004$ & $<.23$ & $<.004$ \\
\hline ZP-46-16-102 & 315042103054301 & $01-12-00$ & $<.002$ & $<.035$ & $<.14$ & $<.04$ & $<.002$ & $<.07$ & $<.29$ & $<.48$ & $<.004$ & $<.23$ & $<.004$ \\
\hline ZR-19-42-612 & 331902097472301 & 04-04-00 & $<.002$ & $<.035$ & $<.06$ & $<.04$ & $<.002$ & $<.07$ & $<.29$ & $<.48$ & $<.004$ & $<.23$ & $<.004$ \\
\hline ZR-19-51-801 & 330906097411701 & 04-04-00 & $<.002$ & $<.035$ & .106 & $<.04$ & $<.002$ & $<.07$ & $<.29$ & $<.48$ & $<.004$ & $<.23$ & $<.004$ \\
\hline ZR-19-51-805 & 330839097412401 & 04-04-00 & $<.002$ & $<.035$ & $<.06$ & $<.04$ & $<.002$ & $<.07$ & $<.29$ & $<.48$ & $<.004$ & $<.23$ & $<.004$ \\
\hline ZR-19-61-705 & 330014097285401 & $01-25-00$ & $<.002$ & $<.035$ & $<.06$ & $<.04$ & $<.002$ & $<.07$ & $<.29$ & $<.48$ & $<.004$ & $<.23$ & $<.004$ \\
\hline ZT-24-50-403 & 331051102511901 & $05-17-00$ & $<.002$ & $<.035$ & $<.06$ & $<.04$ & $<.002$ & $<.07$ & $<.29$ & $<.48$ & $<.004$ & $<.23$ & $<.004$ \\
\hline ZT-27-02-103 & 325819102501601 & $05-23-00$ & $<.002$ & $<.035$ & .450 & $<.04$ & $<.002$ & $<.07$ & $<.29$ & $<.48$ & $<.004$ & $<.23$ & $<.004$ \\
\hline ZT-27-02-105 & 325819102503701 & $05-23-00$ & $<.002$ & $<.035$ & $<.23$ & $<.04$ & $<.002$ & $<.07$ & $<.29$ & $<.48$ & $<.004$ & $<.23$ & $<.004$ \\
\hline
\end{tabular}


Table 5. Concentrations of soluble pesticides in public water-supply wells sampled in Texas, November 1999-January 2001—Continued

\begin{tabular}{|c|c|c|c|c|c|c|c|c|c|c|c|c|}
\hline Local identifier & $\begin{array}{l}\text { Station } \\
\text { number }\end{array}$ & Date & $\begin{array}{c}\text { Dacthal } \\
\text { mono-acid } \\
(\mu \mathrm{g} / \mathrm{L})\end{array}$ & $\begin{array}{l}2,4-D \\
(\mu \mathrm{g} / L)\end{array}$ & $\begin{array}{c}2,4-D B \\
(\mu \mathrm{g} / \mathrm{L})\end{array}$ & $\begin{array}{l}\text { DCPA } \\
(\mu \mathbf{g} / \mathrm{L})\end{array}$ & $\begin{array}{l}\text { Deethyl- } \\
\text { atrazine } \\
(\mu \mathrm{g} / \mathrm{L})\end{array}$ & $\begin{array}{c}\text { Dia- } \\
\text { zinon } \\
(\mu \mathrm{g} / \mathrm{L})\end{array}$ & $\begin{array}{c}\text { Di- } \\
\text { camba } \\
(\mu \mathrm{g} / \mathrm{L})\end{array}$ & $\begin{array}{l}\text { Dichlo- } \\
\text { benil } \\
(\mu \mathrm{g} / \mathrm{L})\end{array}$ & $\begin{array}{c}\text { Dichloro- } \\
\text { prop } \\
(\mu \mathrm{g} / \mathrm{L})\end{array}$ & $\begin{array}{c}\text { Dieldrin } \\
(\mu \mathrm{g} / \mathrm{L})\end{array}$ \\
\hline AB-27-37-103 & 322805102283801 & $05-17-00$ & $<0.039$ & $<0.11$ & $<0.1$ & $<0.002$ & $<0.002$ & $<0.002$ & $<0.043$ & $<0.07$ & $<0.032$ & $<0.001$ \\
\hline AB-27-37-105 & 322826102280101 & $05-17-00$ & $<.039$ & $<.11$ & $<.1$ & $<.002$ & $<.002$ & $<.005$ & $<.043$ & $<.07$ & $<.032$ & $<.001$ \\
\hline $\mathrm{AK}-06-52-506$ & 351024101334401 & $05-04-00$ & $<.039$ & $<.12$ & $<.1$ & $<.002$ & $<.002$ & $<.002$ & $<.043$ & $<.07$ & $<.032$ & $<.001$ \\
\hline AP-59-62-403 & 300259096195001 & 04-03-00 & $<.039$ & $<.11$ & $<.1$ & $<.002$ & $<.002$ & $<.002$ & $<.043$ & $<.07$ & $<.032$ & $<.001$ \\
\hline AP-66-16-407 & 294903096061401 & $04-21-00$ & $<.039$ & $<.11$ & $<.1$ & $<.002$ & $<.002$ & $<.002$ & $<.043$ & $<.07$ & $<.032$ & $<.001$ \\
\hline AR-10-51-408 & 341229102435401 & $05-01-00$ & $<.039$ & $<.11$ & $<.1$ & $<.002$ & E.004 & $<.002$ & $<.043$ & $<.07$ & $<.032$ & $<.001$ \\
\hline AR-24-09-604 & 335000102545701 & 05-09-00 & $<.039$ & $<.14$ & $<.1$ & $<.002$ & $<.002$ & $<.002$ & $<.043$ & $<.07$ & $<.032$ & $<.001$ \\
\hline AT-58-62-206 & 300646097193001 & $12-10-99$ & $<.039$ & $<.11$ & $<.48$ & $<.002$ & E.004 & $<.002$ & $<.043$ & $<.07$ & $<.032$ & $<.001$ \\
\hline AU-21-30-389 & 333612099163101 & $05-02-00$ & $<.039$ & $<.11$ & $<.1$ & $<.002$ & E.015 & $<.002$ & $<.043$ & $<.07$ & $<.032$ & $<.001$ \\
\hline$A X-58-04-604$ & 305718097314401 & $03-22-00$ & $<.039$ & $<.11$ & $<.1$ & $<.002$ & E.024 & $<.002$ & $<.043$ & $<.07$ & $<.032$ & $<.001$ \\
\hline AX-58-04-623 & 305624097321101 & $03-22-00$ & $<.039$ & $<.11$ & $<.1$ & $<.002$ & $<.002$ & $<.002$ & $<.043$ & $<.07$ & $<.032$ & $<.001$ \\
\hline AY-68-35-105 & 292842098425001 & $03-06-00$ & $<.039$ & $<.11$ & $<.1$ & $<.002$ & $<.002$ & $<.002$ & $<.043$ & $<.07$ & $<.032$ & $<.001$ \\
\hline AZ-57-45-612 & 301923098225401 & $04-18-00$ & $<.039$ & $<.11$ & $<.1$ & $<.002$ & $<.002$ & $<.002$ & $<.043$ & $<.07$ & $<.032$ & $<.001$ \\
\hline AZ-57-45-811 & 301633098252901 & $03-29-00$ & $<.039$ & $<.11$ & $<.1$ & $<.002$ & $<.002$ & $<.002$ & $<.043$ & $<.07$ & $<.032$ & $<.001$ \\
\hline BD-16-40-709 & 332301094061701 & 01-18-01 & $<.07$ & $<.11$ & $<.1$ & $<.003$ & $<.006$ & $<.005$ & $<.043$ & $<.049$ & $<.05$ & $<.0048$ \\
\hline BK-73-47-504 & 291911103124301 & $12-28-00$ & $<.07$ & $<.11$ & $<.1$ & $<.003$ & $<.006$ & $<.005$ & $<.043$ & $<.049$ & $<.05$ & $<.0048$ \\
\hline BK-73-52-905 & 290819103312601 & $12-28-00$ & $<.07$ & $<.11$ & $<.1$ & $<.003$ & $<.006$ & $<.005$ & $<.043$ & $<.049$ & $<.05$ & $<.0048$ \\
\hline BL-11-38-334 & 342841101155301 & $05-01-00$ & $<.039$ & $<.11$ & $<.1$ & $<.002$ & $<.002$ & $<.002$ & $<.043$ & $<.07$ & $<.032$ & $<.001$ \\
\hline BL-11-48-507 & 341919101035201 & $05-02-00$ & $<.039$ & $<.11$ & $<.1$ & $<.002$ & $<.002$ & $<.002$ & $<.043$ & $<.07$ & $<.032$ & $<.001$ \\
\hline BL-12-41-204 & 342109100570301 & $05-02-00$ & $<.039$ & $<.11$ & $<.1$ & $<.002$ & E.010 & $<.002$ & $<.043$ & $<.07$ & $<.032$ & $<.001$ \\
\hline BR-41-01-244 & 315849098552101 & $04-05-00$ & $<.039$ & $<.11$ & $<.1$ & $<.002$ & $<.002$ & $<.002$ & $<.043$ & $<.07$ & $<.032$ & $<.001$ \\
\hline BT-57-14-403 & 304939098202401 & 03-08-00 & $<.039$ & $<.11$ & $<.1$ & $<.002$ & $<.002$ & $<.002$ & $<.043$ & $<.07$ & $<.032$ & $<.001$ \\
\hline BT-57-14-404 & 304927098211201 & $03-08-00$ & $<.039$ & $<.11$ & $<.1$ & $<.002$ & $<.002$ & $<.002$ & $<.043$ & $<.07$ & $<.032$ & $<.001$ \\
\hline BT-57-21-607 & 304125098224001 & $03-28-00$ & $<.039$ & $<.11$ & $<.1$ & $<.002$ & $<.002$ & $<.002$ & $<.043$ & $<.07$ & $<.032$ & $<.001$ \\
\hline BT-57-21-608 & 304121098224201 & $03-28-00$ & $<.039$ & $<.11$ & $<.1$ & $<.002$ & $<.002$ & $<.002$ & $<.043$ & $<.07$ & $<.032$ & $<.001$ \\
\hline BT-57-23-110 & 304406098130801 & $03-29-00$ & $<.039$ & $<.11$ & $<.1$ & $<.002$ & E.008 & $<.002$ & $<.043$ & $<.07$ & $<.032$ & $<.001$ \\
\hline BT-57-30-101 & 303548098211701 & $03-01-00$ & $<.039$ & $<.11$ & $<.1$ & $<.002$ & E.007 & $<.002$ & $<.043$ & $<.07$ & $<.032$ & $<.001$ \\
\hline BU-67-10-910 & 294528097464401 & $03-21-00$ & $<.039$ & $<.11$ & $<.1$ & $<.002$ & E.054 & E.003 & $<.043$ & $<.07$ & $<.032$ & $<.001$ \\
\hline
\end{tabular}


Table 5. Concentrations of soluble pesticides in public water-supply wells sampled in Texas, November 1999-January 2001-Continued

\begin{tabular}{|c|c|c|c|c|c|c|c|c|c|c|c|c|}
\hline Local identifier & $\begin{array}{l}\text { Station } \\
\text { number }\end{array}$ & Date & $\begin{array}{c}\text { Dacthal } \\
\text { mono-acid } \\
(\mu \mathrm{g} / \mathrm{L})\end{array}$ & $\begin{array}{l}\text { 2,4-D } \\
(\mu g / L)\end{array}$ & $\begin{array}{c}\text { 2,4-DB } \\
(\mu \mathrm{g} / \mathrm{L})\end{array}$ & $\begin{array}{l}\text { DCPA } \\
(\mu \mathrm{g} / \mathrm{L})\end{array}$ & $\begin{array}{l}\text { Deethyl- } \\
\text { atrazine } \\
(\mu \mathrm{g} / \mathrm{L})\end{array}$ & $\begin{array}{l}\text { Dia- } \\
\text { zinon } \\
(\mu \mathrm{g} / \mathrm{L})\end{array}$ & $\begin{array}{c}\text { Di- } \\
\text { camba } \\
(\mu \mathrm{g} / \mathrm{L})\end{array}$ & $\begin{array}{c}\text { Dichlo- } \\
\text { benil } \\
(\mu \mathrm{g} / \mathrm{L})\end{array}$ & $\begin{array}{l}\text { Dichloro- } \\
\text { prop } \\
(\mu \mathrm{g} / \mathrm{L})\end{array}$ & $\begin{array}{c}\text { Dieldrin } \\
(\mu \mathrm{g} / \mathrm{L})\end{array}$ \\
\hline BX-30-55-936 & 320939099093801 & $12-18-00$ & $<0.07$ & $<0.273$ & $<0.1$ & $<0.003$ & $<0.006$ & $<0.005$ & $<0.043$ & $<0.049$ & $<0.05$ & $<0.0048$ \\
\hline DA-06-28-202 & 353704101343701 & $05-03-00$ & $<.039$ & $<.11$ & $<.1$ & $<.002$ & $<.002$ & $<.002$ & $<.043$ & $<.07$ & $<.032$ & $<.001$ \\
\hline DL-14-62-604 & 340406098160701 & $05-10-00$ & $<.039$ & $<.11$ & $<.1$ & $<.002$ & $<.002$ & $<.002$ & $<.043$ & $<.07$ & $<.032$ & $<.001$ \\
\hline DP-24-18-308 & 334352102450601 & 05-09-00 & $<.039$ & $<.11$ & $<.1$ & $<.002$ & $<.002$ & $<.002$ & $<.043$ & $<.07$ & $<.032$ & $<.001$ \\
\hline DP-25-24-304 & 334245103011001 & $05-08-00$ & $<.039$ & $<.29$ & $<.1$ & $<.002$ & $<.002$ & $<.002$ & $<.043$ & $<.07$ & $<.032$ & $<.001$ \\
\hline DU-12-06-609 & 345658100155501 & $05-08-00$ & $<.039$ & $<.13$ & $<.1$ & $<.002$ & $<.002$ & $<.002$ & $<.043$ & $<.07$ & $<.032$ & $<.001$ \\
\hline DX-68-05-621 & 295520098242301 & $03-22-00$ & $<.039$ & $<.11$ & $<.1$ & $<.002$ & $<.002$ & .014 & $<.043$ & $<.07$ & $<.032$ & $<.001$ \\
\hline DX-68-06-405 & 295528098222301 & $03-22-00$ & $<.039$ & $<.11$ & $<.1$ & $<.002$ & $<.002$ & $<.002$ & $<.043$ & $<.07$ & $<.032$ & $<.001$ \\
\hline DY-41-13-307 & 315105098241401 & $04-05-00$ & $<.039$ & $<.11$ & $<.1$ & $<.002$ & $<.002$ & $<.002$ & $<.043$ & $<.07$ & $<.032$ & $<.001$ \\
\hline HH-45-35-505 & 312715102402201 & $01-12-00$ & $<.039$ & $<.11$ & $<.25$ & $<.002$ & $<.002$ & $<.002$ & $<.043$ & $<.07$ & $<.032$ & $<.001$ \\
\hline HP-12-62-802 & 340210100184901 & $05-05-00$ & $<.039$ & $<.11$ & $<.1$ & $<.002$ & E.008 & $<.002$ & $<.043$ & -- & $<.032$ & $<.001$ \\
\hline HS-28-09-404 & 324810101591201 & $05-16-00$ & $<.039$ & $<.11$ & $<.1$ & $<.002$ & $<.002$ & $<.002$ & $<.043$ & $<.07$ & $<.032$ & $<.001$ \\
\hline HT-07-63-204 & 350607102103401 & $05-03-00$ & $<.039$ & $<.11$ & $<.1$ & $<.002$ & $<.002$ & $<.002$ & $<.043$ & $<.07$ & $<.032$ & $<.001$ \\
\hline HZ-77-34-606 & 282608099455301 & $03-28-00$ & $<.039$ & $<.11$ & $<.1$ & $<.002$ & $<.002$ & $<.002$ & $<.043$ & $<.07$ & $<.032$ & $<.001$ \\
\hline JA-05-57-613 & 350254100535501 & 05-04-00 & $<.039$ & $<.11$ & $<.1$ & $<.002$ & $<.002$ & $<.002$ & $<.043$ & $<.07$ & $<.032$ & $<.001$ \\
\hline JA-12-12-404 & 344910100360401 & $05-02-00$ & $<.039$ & $<.11$ & $<.1$ & $<.002$ & $<.002$ & $<.002$ & $<.043$ & $<.07$ & $<.032$ & $<.001$ \\
\hline JH-45-05-924 & 315245102240201 & $05-25-00$ & $<.039$ & $<.11$ & $<.1$ & $<.002$ & $<.002$ & $<.002$ & $<.043$ & $<.78$ & $<.032$ & $<.001$ \\
\hline JL-49-04-116 & 315757106370201 & $01-06-00$ & $<.039$ & $<.11$ & $<.15$ & $<.002$ & $<.002$ & $<.002$ & $<.043$ & $<.07$ & $<.032$ & $<.001$ \\
\hline JL-49-04-423 & 315708106362301 & 01-03-00 & $<.039$ & $<.11$ & $<.45$ & $<.002$ & $<.002$ & $<.002$ & $<.043$ & $<.07$ & $<.032$ & $<.001$ \\
\hline JL-49-04-428 & 315517106361401 & $01-06-00$ & $<.039$ & $<.11$ & $<.27$ & $<.002$ & $<.002$ & $<.002$ & $<.043$ & $<.07$ & $<.032$ & $<.001$ \\
\hline JL-49-04-492 & 315623106360601 & $01-06-00$ & $<.039$ & $<.11$ & $<.25$ & $<.002$ & $<.002$ & $<.002$ & $<.043$ & $<.07$ & $<.032$ & $<.001$ \\
\hline JL-49-22-626 & 314145106163601 & $01-04-00$ & $<.039$ & $<.11$ & $<.22$ & $<.002$ & $<.002$ & $<.002$ & $<.043$ & $<.07$ & $<.032$ & $<.001$ \\
\hline JL-49-24-423 & 314107106063301 & $01-07-00$ & $<.039$ & $<.11$ & $<.32$ & $<.002$ & $<.002$ & $<.002$ & $<.043$ & $<.07$ & $<.032$ & $<.001$ \\
\hline JL-49-40-502 & 312648106044701 & 01-04-00 & $<.039$ & $<.11$ & $<.32$ & $<.002$ & $<.002$ & $<.002$ & $<.043$ & $<.07$ & $<.032$ & $<.001$ \\
\hline$J Y-65-26-520$ & 293314095474702 & 04-03-00 & $<.039$ & $<.11$ & $<.1$ & $<.002$ & $<.002$ & $<.002$ & $<.043$ & $<.07$ & $<.032$ & $<.001$ \\
\hline KD-27-01-612 & 325523102542701 & $05-22-00$ & $<.039$ & $<.11$ & $<.1$ & $<.002$ & $<.002$ & $<.002$ & $<.043$ & $<.07$ & $<.032$ & $<.001$ \\
\hline KK-57-42-709 & 301545098502801 & 07-06-00 & $<.039$ & $<.11$ & $<.1$ & $<.002$ & $<.002$ & $<.002$ & $<.043$ & $<.07$ & $<.032$ & $<.001$ \\
\hline KK-57-50-325 & 301357098471901 & $03-28-00$ & $<.039$ & $<.11$ & $<.1$ & $<.002$ & E.011 & $<.002$ & $<.043$ & $<.07$ & $<.032$ & $<.001$ \\
\hline KK-57-51-303 & 301411098383101 & $03-21-00$ & $<.039$ & $<.11$ & $<.1$ & $<.002$ & E.082 & $<.002$ & $<.043$ & $<.07$ & $<.032$ & $<.001$ \\
\hline KK-57-52-107 & 301430098363401 & $03-21-00$ & $<.039$ & $<.11$ & $<.1$ & $<.002$ & $<.002$ & $<.002$ & $<.043$ & $<.07$ & $<.032$ & $<.001$ \\
\hline
\end{tabular}


Table 5. Concentrations of soluble pesticides in public water-supply wells sampled in Texas, November 1999-January 2001—Continued

\begin{tabular}{|c|c|c|c|c|c|c|c|c|c|c|c|c|}
\hline Local identifier & $\begin{array}{l}\text { Station } \\
\text { number }\end{array}$ & Date & $\begin{array}{l}\text { Dacthal } \\
\text { mono-acid } \\
(\mu \mathrm{g} / \mathrm{L})\end{array}$ & $\begin{array}{l}2,4-D \\
(\mu \mathrm{g} / L)\end{array}$ & $\begin{array}{c}2,4-D B \\
(\mu \mathrm{g} / L)\end{array}$ & $\begin{array}{l}\text { DCPA } \\
(\mu \mathrm{g} / \mathrm{L})\end{array}$ & $\begin{array}{c}\text { Deethyl- } \\
\text { atrazine } \\
(\mu \mathrm{g} / \mathrm{L})\end{array}$ & $\begin{array}{l}\text { Dia- } \\
\text { zinon } \\
(\mu \mathrm{g} / \mathrm{L})\end{array}$ & $\begin{array}{c}\text { Di- } \\
\text { camba } \\
(\mu \mathrm{g} / \mathrm{L})\end{array}$ & $\begin{array}{c}\text { Dichlo- } \\
\text { benil } \\
(\mu \mathrm{g} / \mathrm{L})\end{array}$ & $\begin{array}{c}\text { Dichloro- } \\
\text { prop } \\
(\mu \mathrm{g} / \mathrm{L})\end{array}$ & $\begin{array}{c}\text { Dieldrin } \\
(\mu \mathrm{g} / \mathrm{L})\end{array}$ \\
\hline KP-79-23-101 & 284256097125001 & $02-29-00$ & $<0.039$ & $<0.11$ & $<0.1$ & $<0.002$ & $<0.002$ & 0.016 & $<0.043$ & $<0.07$ & $<0.032$ & $<0.001$ \\
\hline $\mathrm{KS}-05-52-105$ & 351428100352601 & 05-04-00 & $<.039$ & $<.11$ & $<.1$ & $<.002$ & $<.002$ & $<.002$ & $<.043$ & $<.07$ & $<.032$ & $<.001$ \\
\hline KT-18-11-405 & 334844096435701 & 04-19-00 & $<.039$ & $<.11$ & $<.1$ & $<.002$ & $<.002$ & $<.002$ & $<.043$ & $<.07$ & $<.032$ & $<.001$ \\
\hline KT-18-11-406 & 334840096434001 & 04-19-00 & $<.039$ & $<.11$ & $<.1$ & $<.002$ & E.009 & $<.002$ & $<.043$ & $<.07$ & $<.032$ & $<.001$ \\
\hline KT-18-28-703 & 333039096362901 & 04-19-00 & $<.039$ & $<.11$ & $<.1$ & $<.002$ & $<.002$ & $<.002$ & $<.043$ & $<.07$ & $<.032$ & $<.001$ \\
\hline KY-11-49-512 & 341116101574601 & $05-04-00$ & $<.039$ & $<.12$ & $<.1$ & $<.002$ & E.046 & $<.002$ & $<.043$ & $<.07$ & $<.032$ & $<.001$ \\
\hline KY-11-51-416 & 341032101423801 & $05-04-00$ & $<.039$ & $<.11$ & $<.1$ & $<.002$ & 1.73 & $<.002$ & $<.043$ & $<.07$ & $<.21$ & $<.001$ \\
\hline KY-11-57-602 & 340345101531801 & $05-03-00$ & $<.039$ & $<.11$ & $<.1$ & $<.002$ & E.016 & $<.002$ & $<.043$ & $<.07$ & $<.032$ & $<.001$ \\
\hline KZ-12-19-104 & 344234100431601 & 05-09-00 & $<.039$ & $<.11$ & $<.1$ & $<.002$ & E.004 & $<.002$ & $<.043$ & $<.07$ & $<.032$ & $<.001$ \\
\hline LD-13-44-907 & 341523099305701 & $12-19-00$ & $<.07$ & $<.272$ & $<.1$ & $<.003$ & E.006 & $<.005$ & $<.043$ & $<.049$ & $<.05$ & $<.0048$ \\
\hline LJ-60-64-807 & 300149095045001 & $05-04-00$ & $<.039$ & $<.11$ & $<.1$ & $<.002$ & $<.002$ & $<.002$ & $<.043$ & $<.07$ & $<.032$ & $<.001$ \\
\hline LP-21-49-317 & 331233099545201 & $05-01-00$ & $<.039$ & $<.11$ & $<.1$ & $<.002$ & $<.002$ & $<.002$ & $<.043$ & $<.07$ & $<.032$ & $<.001$ \\
\hline LR-67-09-112 & 295137097585801 & $03-21-00$ & $<.039$ & $<.11$ & $<.1$ & $<.002$ & E.004 & $<.002$ & $<.043$ & $<.07$ & $<.032$ & $<.001$ \\
\hline LX-24-15-612 & 334857102092301 & $05-11-00$ & $<.039$ & $<.12$ & $<.1$ & $<.002$ & E.012 & $<.002$ & .038 & $<.07$ & $<.032$ & $<.001$ \\
\hline LX-24-29-306 & 333536102223101 & 05-09-00 & $<.039$ & $<.11$ & $<.1$ & $<.002$ & E.027 & $<.002$ & $<.043$ & $<.07$ & $<.032$ & $<.001$ \\
\hline LX-24-37-405 & 332714102285401 & $05-16-00$ & $<.039$ & $<.11$ & $<.1$ & $<.002$ & E.007 & $<.002$ & $<.043$ & $<.07$ & $<.032$ & $<.001$ \\
\hline LX-24-40-405 & 332624102072901 & $05-17-00$ & $<.039$ & $<.11$ & $<.1$ & $<.002$ & $<.002$ & $<.002$ & $<.043$ & $<.07$ & $<.032$ & $<.001$ \\
\hline LY-32-26-706 & 323204097500301 & $03-22-00$ & $<.039$ & $<.11$ & $<.1$ & $<.002$ & $<.002$ & $<.002$ & $<.043$ & $<.07$ & $<.032$ & $<.001$ \\
\hline LY-32-34-208 & 322958097481301 & $03-21-00$ & $<.039$ & $<.11$ & $<.14$ & $<.002$ & $<.002$ & $<.002$ & $<.043$ & $<.07$ & $<.032$ & $<.001$ \\
\hline LY-32-34-303 & 322928097461701 & 04-03-00 & $<.039$ & $<.11$ & $<.1$ & $<.002$ & $<.002$ & $<.002$ & $<.043$ & $<.07$ & $<.032$ & $<.001$ \\
\hline LY-32-34-510 & 322537097485701 & $03-21-00$ & $<.039$ & $<.11$ & $<.1$ & $<.002$ & $<.002$ & $<.002$ & $<.043$ & $<.07$ & $<.032$ & $<.001$ \\
\hline LY-32-34-609 & 322626097454801 & $03-21-00$ & $<.039$ & $<.11$ & $<.1$ & $<.002$ & $<.002$ & $<.002$ & $<.043$ & $<.07$ & $<.032$ & $<.001$ \\
\hline PB-28-62-105 & 320649101215501 & 04-26-00 & $<.039$ & $<.11$ & $<.1$ & $<.002$ & $<.002$ & $<.002$ & $<.043$ & $<.07$ & $<.032$ & $<.001$ \\
\hline PK-43-50-216 & 311422100490201 & $12-20-00$ & $<.07$ & $<.278$ & $<.1$ & $<.003$ & $<.006$ & $<.005$ & $<.043$ & $<.049$ & $<.05$ & $<.0048$ \\
\hline PS-52-02-404 & 305502103504101 & $01-10-00$ & $<.039$ & $<.14$ & $<.28$ & $<.002$ & $<.002$ & $<.002$ & $<.043$ & $<.07$ & $<.032$ & $<.001$ \\
\hline PU-84-43-504 & 271841098402801 & 04-04-00 & $<.039$ & $<.11$ & $<.1$ & $<.002$ & $<.002$ & .009 & $<.043$ & $<.07$ & $<.032$ & $<.001$ \\
\hline PX-32-47-109 & 322201097141601 & $03-20-00$ & $<.039$ & $<.11$ & $<.1$ & $<.002$ & $<.002$ & $<.002$ & $<.043$ & $<.07$ & $<.032$ & $<.001$ \\
\hline PZ-79-10-807 & 284713097493901 & $02-23-00$ & $<.039$ & $<.11$ & $<.1$ & $<.002$ & $<.002$ & $<.002$ & $<.043$ & $<.07$ & $<.032$ & $<.001$ \\
\hline RS-21-35-103 & 332854099441101 & $05-01-00$ & $<.039$ & $<.11$ & $<.1$ & $<.002$ & E.106 & $<.002$ & $<.043$ & $<.07$ & $<.032$ & $<.001$ \\
\hline RU-10-53-312 & 341418102243401 & $05-02-00$ & $<.039$ & $<.11$ & $<.1$ & $<.002$ & E.105 & $<.002$ & $<.043$ & $<.07$ & $<.032$ & $<.001$ \\
\hline
\end{tabular}


Table 5. Concentrations of soluble pesticides in public water-supply wells sampled in Texas, November 1999-January 2001-Continued

\begin{tabular}{|c|c|c|c|c|c|c|c|c|c|c|c|c|}
\hline Local identifier & $\begin{array}{l}\text { Station } \\
\text { number }\end{array}$ & Date & $\begin{array}{c}\text { Dacthal } \\
\text { mono-acid } \\
(\mu \mathrm{g} / \mathrm{L})\end{array}$ & $\begin{array}{l}\text { 2,4-D } \\
(\mu g / L)\end{array}$ & $\begin{array}{c}\text { 2,4-DB } \\
(\mu \mathrm{g} / \mathrm{L})\end{array}$ & $\begin{array}{l}\text { DCPA } \\
(\mu \mathrm{g} / \mathrm{L})\end{array}$ & $\begin{array}{l}\text { Deethyl- } \\
\text { atrazine } \\
(\mu \mathrm{g} / \mathrm{L})\end{array}$ & $\begin{array}{l}\text { Dia- } \\
\text { zinon } \\
(\mu \mathrm{g} / \mathrm{L})\end{array}$ & $\begin{array}{c}\text { Di- } \\
\text { camba } \\
(\mu \mathrm{g} / \mathrm{L})\end{array}$ & $\begin{array}{c}\text { Dichlo- } \\
\text { benil } \\
(\mu \mathrm{g} / \mathrm{L})\end{array}$ & $\begin{array}{l}\text { Dichloro- } \\
\text { prop } \\
(\mu \mathrm{g} / \mathrm{L})\end{array}$ & $\begin{array}{c}\text { Dieldrin } \\
(\mu \mathrm{g} / \mathrm{L})\end{array}$ \\
\hline RU-10-60-606 & 340427102302801 & $05-02-00$ & $<0.039$ & $<0.11$ & $<.1$ & $<0.002$ & E0.004 & $<0.002$ & $<0.043$ & $<0.07$ & $<0.032$ & $<0.001$ \\
\hline RW-41-63-520 & 310238098104001 & $03-23-00$ & $<.039$ & $<.11$ & $<.1$ & $<.002$ & $<.002$ & $<.002$ & $<.043$ & $<.07$ & $<.032$ & $<.001$ \\
\hline SK-57-19-201 & 304323098421701 & $03-08-00$ & $<.039$ & $<.11$ & $<.1$ & $<.002$ & $<.002$ & $<.002$ & $<.043$ & $<.07$ & $<.032$ & $<.001$ \\
\hline SK-57-34-201 & 303001098485201 & 03-09-00 & $<.039$ & $<.11$ & $<.1$ & $<.002$ & $<.002$ & $<.002$ & $<.043$ & $<.07$ & $<.032$ & $<.001$ \\
\hline SP-23-17-417 & 334043101585701 & $05-10-00$ & $<.039$ & $<.11$ & $<.1$ & $<.002$ & E.039 & $<.002$ & $<.043$ & $<.07$ & $<.032$ & $<.001$ \\
\hline SP-23-18-107 & 334306101504101 & $05-10-00$ & $<.039$ & $<.15$ & $<.1$ & $<.002$ & E.089 & $<.002$ & $<.043$ & $<.07$ & $<.032$ & $<.001$ \\
\hline SP-23-19-812 & 333955101404601 & $05-10-00$ & $<.039$ & $<.12$ & $<.1$ & $<.002$ & $<.002$ & $<.002$ & $<.043$ & $<.07$ & $<.032$ & $<.001$ \\
\hline SP-24-24-214 & 334419102045201 & $05-18-00$ & $<.039$ & $<.11$ & $<.1$ & $<.002$ & E.006 & $<.002$ & $<.043$ & $<.07$ & $<.032$ & $<.001$ \\
\hline SR-23-41-602 & 331933101543701 & $05-15-00$ & $<.039$ & $<.11$ & $<.1$ & $<.002$ & E.022 & $<.002$ & $<.043$ & $<.07$ & $<.032$ & $<.001$ \\
\hline SS-42-52-504 & 311202099321401 & $03-01-00$ & $<.039$ & $<.11$ & $<.1$ & $<.002$ & $<.002$ & $<.002$ & $<.043$ & $<.07$ & $<.032$ & $<.001$ \\
\hline ST-40-39-304 & 312745097083301 & $03-23-00$ & $<.039$ & $<.11$ & $<.1$ & $<.002$ & $<.002$ & $<.002$ & $<.043$ & $<.07$ & $<.032$ & $<.001$ \\
\hline SY-27-39-505 & 322555102105501 & $05-24-00$ & $<.039$ & $<.11$ & $<.1$ & $<.002$ & $<.002$ & $<.002$ & $<.043$ & $<.07$ & $<.032$ & $<.001$ \\
\hline SY $-28-42-803$ & 321619101495001 & $05-23-00$ & $<.039$ & $<.11$ & $<.1$ & $<.002$ & $<.002$ & $<.002$ & $<.043$ & $<.07$ & $<.032$ & $<.001$ \\
\hline SY-28-50-908 & 320816101474601 & $05-23-00$ & $<.039$ & $<.11$ & $<.1$ & $<.002$ & $<.002$ & .008 & $<.043$ & $<.14$ & $<.032$ & $<.001$ \\
\hline TB-76-03-605 & 285657100373801 & $03-28-00$ & $<.039$ & $<.11$ & $<.1$ & $<.002$ & $<.002$ & $<.002$ & $<.043$ & $<.07$ & $<.032$ & $<.001$ \\
\hline TJ-27-63-712 & 320143102123101 & $12-20-00$ & $<.07$ & $<.11$ & $<.1$ & $<.003$ & $<.006$ & $<.005$ & $<.043$ & $<.049$ & $<.05$ & $<.0048$ \\
\hline TJ-28-57-702 & 320221101594901 & $05-25-00$ & $<.039$ & $<.11$ & $<.1$ & $<.002$ & $<.002$ & $<.002$ & $<.043$ & $<.07$ & $<.032$ & $<.001$ \\
\hline TJ-28-57-903 & 320010101523701 & $05-23-00$ & $<.039$ & $<.11$ & $<.1$ & $<.002$ & $<.002$ & $<.002$ & $<.043$ & $<.07$ & $<.032$ & $<.001$ \\
\hline TJ-45-07-407 & 315643102131101 & $05-24-00$ & $<.039$ & $<.11$ & $<.1$ & $<.002$ & $<.002$ & $<.002$ & $<.043$ & $<.07$ & $<.032$ & $<.001$ \\
\hline TJ-45-07-606 & 315702102075401 & $05-25-00$ & $<.039$ & $<.11$ & $<.1$ & $<.002$ & $<.002$ & .004 & $<.043$ & $<.07$ & $<.032$ & $<.001$ \\
\hline TS-60-36-510 & 302701095331201 & 04-20-00 & $<.039$ & $<.11$ & $<.1$ & $<.002$ & $<.002$ & $<.002$ & $<.043$ & $<.07$ & $<.032$ & $<.001$ \\
\hline TW-22-02-714 & 335416100502101 & $05-05-00$ & $<.039$ & $<.11$ & $<.1$ & $<.002$ & $<.002$ & $<.002$ & $<.043$ & $<.07$ & $<.032$ & $<.001$ \\
\hline TW-22-02-716 & 335449100513101 & $12-19-00$ & $<.07$ & $<.22$ & $<.1$ & $<.003$ & $<.006$ & $<.005$ & $<.043$ & $<.049$ & $<.05$ & $<.0048$ \\
\hline UJ-62-49-712 & 300906093585201 & $04-28-00$ & $<.039$ & $<.11$ & $<.1$ & $<.002$ & $<.002$ & $<.002$ & $<.043$ & $<.07$ & $<.032$ & $<.001$ \\
\hline UK-31-05-501 & 325545098255501 & $03-22-00$ & $<.039$ & $<.11$ & $<.1$ & $<.002$ & E.041 & $<.002$ & $<.043$ & $<.07$ & $<.032$ & $<.001$ \\
\hline UK-31-24-501 & 324210098034701 & $03-22-00$ & $<.039$ & $<.11$ & $<.1$ & $<.002$ & $<.002$ & $<.002$ & $<.043$ & $<.07$ & $<.032$ & $<.001$ \\
\hline UL-35-48-202 & 322207094034501 & $04-25-00$ & $<.039$ & $<.11$ & $<.1$ & $<.002$ & $<.002$ & $<.002$ & $<.043$ & $<.07$ & $<.032$ & $<.001$ \\
\hline UP-32-10-604 & 324926097455001 & 02-09-00 & $<.039$ & $<.11$ & $<.35$ & $<.002$ & $<.002$ & $<.002$ & $<.043$ & $<.07$ & $<.032$ & $<.001$ \\
\hline UP-32-11-103 & 325005097440101 & 02-09-00 & $<.039$ & $<.11$ & $<.32$ & $<.002$ & $<.002$ & $<.002$ & $<.043$ & $<.07$ & $<.032$ & $<.001$ \\
\hline UP-32-11-709 & 324649097442001 & 02-09-00 & $<.039$ & $<.11$ & $<.4$ & $<.002$ & $<.002$ & $<.002$ & $<.043$ & $<.07$ & $<.032$ & $<.001$ \\
\hline
\end{tabular}


Table 5. Concentrations of soluble pesticides in public water-supply wells sampled in Texas, November 1999-January 2001—Continued

\begin{tabular}{|c|c|c|c|c|c|c|c|c|c|c|c|c|}
\hline Local identifier & $\begin{array}{l}\text { Station } \\
\text { number }\end{array}$ & Date & $\begin{array}{c}\text { Dacthal } \\
\text { mono-acid } \\
(\mu \mathrm{g} / \mathrm{L})\end{array}$ & $\begin{array}{c}2,4-D \\
(\mu g / L)\end{array}$ & $\begin{array}{c}2,4-D B \\
(\mu \mathrm{g} / \mathrm{L})\end{array}$ & $\begin{array}{l}\text { DCPA } \\
(\mu \mathrm{g} / \mathrm{L})\end{array}$ & $\begin{array}{c}\text { Deethyl- } \\
\text { atrazine } \\
(\mu \mathrm{g} / \mathrm{L})\end{array}$ & $\begin{array}{l}\text { Dia- } \\
\text { zinon } \\
(\mu \mathrm{g} / \mathrm{L})\end{array}$ & $\begin{array}{c}\text { Di- } \\
\text { camba } \\
(\mu \mathrm{g} / \mathrm{L})\end{array}$ & $\begin{array}{c}\text { Dichlo- } \\
\text { benil } \\
(\mu \mathrm{g} / \mathrm{L})\end{array}$ & $\begin{array}{c}\text { Dichloro- } \\
\text { prop } \\
(\mu \mathrm{g} / \mathrm{L})\end{array}$ & $\begin{array}{c}\text { Dieldrin } \\
(\mu \mathbf{g} / L)\end{array}$ \\
\hline UP-32-11-801 & 324548097411701 & $02-10-00$ & $<0.039$ & $<0.11$ & $<0.59$ & $<0.002$ & $<0.002$ & $<0.002$ & $<0.043$ & $<0.07$ & $<0.032$ & $<0.001$ \\
\hline UP-32-25-602 & 323439097524301 & 02-09-00 & $<.039$ & $<.11$ & $<.35$ & $<.002$ & $<.002$ & $<.002$ & $<.043$ & $<.07$ & $<.032$ & $<.001$ \\
\hline UP-32-28-403 & 323356097361801 & 04-10-00 & $<.039$ & $<.11$ & $<.1$ & $<.002$ & $<.002$ & $<.002$ & $<.043$ & $<.07$ & $<.032$ & $<.001$ \\
\hline US-53-08-601 & 305723102015801 & $03-14-00$ & $<.039$ & $<.11$ & $<.1$ & $<.002$ & $<.002$ & $<.002$ & $<.043$ & $<.07$ & $<.032$ & $<.001$ \\
\hline US-54-18-503 & 304131101492401 & $03-14-00$ & $<.039$ & $<.11$ & $<.1$ & $<.002$ & $<.002$ & .025 & $<.043$ & $<.07$ & $<.032$ & $<.001$ \\
\hline UT-60-24-110 & 304251095060001 & 04-26-00 & $<.039$ & $<.11$ & $<.1$ & $<.002$ & $<.002$ & $<.002$ & $<.043$ & $<.07$ & $<.032$ & $<.001$ \\
\hline UY-07-64-914 & 350224102020301 & $05-03-00$ & $<.039$ & $<.11$ & $<.1$ & $<.002$ & E.038 & $<.002$ & $<.043$ & $<.07$ & $<.032$ & $<.001$ \\
\hline UZ-44-37-505 & 312656101265101 & $01-13-00$ & $<.039$ & $<.11$ & $<.1$ & $<.002$ & $<.002$ & $<.002$ & $<.043$ & $<.07$ & $<.032$ & $<.001$ \\
\hline WB-16-18-704 & 333942094503001 & 04-26-00 & $<.039$ & $<.11$ & $<.1$ & $<.002$ & $<.002$ & $<.002$ & $<.043$ & $<.07$ & $<.032$ & $<.001$ \\
\hline WD-46-46-210 & 312041103174201 & $01-11-00$ & $<.039$ & $<.11$ & $<.21$ & $<.002$ & $<.002$ & $<.002$ & $<.043$ & $<.21$ & $<.032$ & $<.001$ \\
\hline WK-39-51-801 & 310956096402901 & 04-20-00 & $<.039$ & $<.11$ & $<.1$ & $<.002$ & $<.002$ & $<.002$ & $<.043$ & $<.07$ & $<.032$ & $<.001$ \\
\hline $\mathrm{WP}-43-31-211$ & 313551100110801 & $04-25-00$ & $<.039$ & $<.11$ & $<.1$ & $<.002$ & E.003 & $<.002$ & $<.043$ & $<.07$ & $<.032$ & $<.001$ \\
\hline WT-37-31-706 & 313110094131201 & $04-27-00$ & $<.039$ & $<.11$ & $<.1$ & $<.002$ & $<.002$ & $<.002$ & $<.043$ & $<.07$ & $<.032$ & $<.001$ \\
\hline XH-34-37-312 & 322832095245401 & $04-25-00$ & $<.039$ & $<.11$ & $<.1$ & $<.002$ & $<.002$ & $<.002$ & $<.043$ & $<.07$ & $<.032$ & $<.001$ \\
\hline XP-43-09-119 & 315033100584001 & 04-26-00 & $<.039$ & $<.11$ & $<.1$ & $<.002$ & $<.002$ & $<.002$ & $<.043$ & $<.07$ & $<.032$ & $<.001$ \\
\hline XR-22-54-201 & 331310100180801 & $05-01-00$ & $<.039$ & $<.11$ & $<.1$ & $<.002$ & $<.002$ & $<.002$ & $<.043$ & $<.07$ & $<.032$ & $<.001$ \\
\hline XT-11-26-923 & 343046101463701 & $05-01-00$ & $<.039$ & $<.11$ & $<.1$ & $<.002$ & E.016 & $<.002$ & $<.043$ & $<.07$ & $<.032$ & $<.001$ \\
\hline XU-32-04-604 & 325525097304801 & $01-05-00$ & $<.039$ & $<.11$ & $<.4$ & $<.002$ & $<.002$ & $<.002$ & $<.043$ & $<.07$ & $<.032$ & $<.001$ \\
\hline XU-32-04-605 & 325525097304802 & $01-05-00$ & $<.039$ & $<.11$ & $<.22$ & $<.002$ & $<.002$ & $<.002$ & $<.043$ & $<.07$ & $<.032$ & $<.001$ \\
\hline XU-32-05-805 & 325335097272401 & 04-03-00 & $<.039$ & $<.11$ & $<.1$ & $<.002$ & $<.002$ & $<.002$ & $<.043$ & $<.07$ & $<.032$ & $<.001$ \\
\hline XU-32-12-307 & 325101097313101 & $03-23-00$ & $<.039$ & $<.11$ & $<.1$ & $<.002$ & $<.002$ & $<.002$ & $<.043$ & $<.07$ & $<.032$ & $<.001$ \\
\hline XU-32-13-405 & 324901097284701 & $01-24-00$ & $<.039$ & $<.11$ & $<.42$ & $<.002$ & $<.002$ & $<.002$ & $<.043$ & $<.07$ & $<.032$ & $<.001$ \\
\hline XU-32-13-503 & 324816097272401 & $01-24-00$ & $<.039$ & $<.11$ & $<.53$ & $<.002$ & $<.002$ & $<.002$ & $<.043$ & $<.07$ & $<.032$ & $<.001$ \\
\hline XU-32-23-701 & 323911097131001 & $03-20-00$ & $<.039$ & $<.11$ & $<.1$ & $<.002$ & $<.002$ & $<.002$ & $<.043$ & $<.07$ & $<.032$ & $<.001$ \\
\hline XY-24-54-606 & 331112102160201 & $05-17-00$ & $<.039$ & $<.11$ & $<.1$ & $<.002$ & $<.002$ & $<.002$ & $<.043$ & $<.07$ & $<.032$ & $<.001$ \\
\hline YB-43-19-701 & 313943100425301 & $01-14-00$ & $<.039$ & $<.11$ & $<.38$ & $<.002$ & $<.002$ & $<.002$ & $<.043$ & $<.07$ & $<.032$ & $<.001$ \\
\hline YB-43-27-201 & 313638100400201 & 01-14-00 & $<.039$ & $<.11$ & $<.33$ & $<.002$ & $<.002$ & $<.002$ & $<.043$ & $<.07$ & $<.032$ & $<.001$ \\
\hline YB-43-44-102 & 312003100370101 & $01-14-00$ & $<.039$ & $<.11$ & $<.25$ & $<.002$ & $<.002$ & $<.002$ & $<.043$ & $<.07$ & $<.032$ & $<.001$ \\
\hline YD-57-40-703 & 302306098050701 & 04-19-00 & $<.039$ & $<.11$ & $<.1$ & $<.002$ & $<.002$ & $<.002$ & $<.043$ & $<.07$ & $<.032$ & $<.001$ \\
\hline YD-58-50-830 & 300938097490601 & 11-03-99 & $<.039$ & $<.11$ & $<.45$ & $<.002$ & $<.002$ & $<.002$ & $<.043$ & $<.07$ & $<.032$ & $<.001$ \\
\hline
\end{tabular}


Table 5. Concentrations of soluble pesticides in public water-supply wells sampled in Texas, November 1999-January 2001-Continued

\begin{tabular}{|c|c|c|c|c|c|c|c|c|c|c|c|c|}
\hline Local identifier & $\begin{array}{l}\text { Station } \\
\text { number }\end{array}$ & Date & $\begin{array}{c}\text { Dacthal } \\
\text { mono-acid } \\
(\mu \mathrm{g} / \mathrm{L})\end{array}$ & $\begin{array}{l}2,4-D \\
(\mu g / L)\end{array}$ & $\begin{array}{c}2,4-D B \\
(\mu \mathrm{g} / L)\end{array}$ & $\begin{array}{l}\text { DCPA } \\
(\mu \mathrm{g} / \mathrm{L})\end{array}$ & $\begin{array}{c}\text { Deethyl- } \\
\text { atrazine } \\
(\mu \mathrm{g} / \mathrm{L})\end{array}$ & $\begin{array}{c}\text { Dia- } \\
\text { zinon } \\
(\mu \mathrm{g} / \mathrm{L})\end{array}$ & $\begin{array}{c}\text { Di- } \\
\text { camba } \\
(\mu \mathrm{g} / \mathrm{L})\end{array}$ & $\begin{array}{c}\text { Dichlo- } \\
\text { benil } \\
(\mu \mathrm{g} / \mathrm{L})\end{array}$ & $\begin{array}{c}\text { Dichloro- } \\
\text { prop } \\
(\mu \mathrm{g} / \mathrm{L})\end{array}$ & $\begin{array}{c}\text { Dieldrin } \\
(\mu \mathrm{g} / \mathrm{L})\end{array}$ \\
\hline YD-58-52-304 & 301305097314601 & $04-27-00$ & $<0.039$ & $<0.11$ & $<0.1$ & $<0.002$ & E0.006 & $<0.002$ & $<0.043$ & $<0.07$ & $<0.032$ & $<0.001$ \\
\hline YP-69-27-107 & 293652099442501 & 10-19-00 & $<.07$ & $<.11$ & $<.1$ & $<.003$ & $<.006$ & $<.005$ & $<.043$ & $<.049$ & $<.05$ & $<.0048$ \\
\hline YP-69-27-402 & 293450099433701 & $10-19-00$ & $<.07$ & $<.11$ & $<.1$ & $<.003$ & $<.006$ & $<.005$ & $<.043$ & $<.049$ & $<.05$ & $<.0048$ \\
\hline YP-69-27-704 & 293037099430301 & $10-18-00$ & $<.07$ & $<.11$ & $<.1$ & $<.003$ & $<.006$ & $<.005$ & $<.043$ & $<.049$ & $<.05$ & $<.0048$ \\
\hline YX-46-32-625 & 313252103010301 & $01-11-00$ & $<.039$ & $<.11$ & $<.41$ & $<.002$ & $<.002$ & $<.002$ & $<.043$ & $<.07$ & $<.032$ & $<.001$ \\
\hline$Y X-46-40-311$ & 312848103012901 & $01-11-00$ & $<.039$ & $<.17$ & $<.22$ & $<.002$ & $<.002$ & $<.002$ & $<.043$ & $<.07$ & $<.032$ & $<.001$ \\
\hline YY-59-54-902 & 300824096152400 & 04-19-00 & $<.039$ & $<.11$ & $<.1$ & $<.002$ & $<.002$ & $<.002$ & $<.043$ & $<.07$ & $<.032$ & $<.001$ \\
\hline ZB-05-29-819 & 353215100261201 & $05-10-00$ & $<.039$ & $<.11$ & $<.1$ & $<.002$ & $<.002$ & $<.002$ & $<.043$ & $<.07$ & $<.032$ & $<.001$ \\
\hline ZB-05-38-512 & 352709100182901 & $05-08-00$ & $<.039$ & $<.11$ & $<.1$ & $<.002$ & E.008 & $<.002$ & $<.043$ & $<.07$ & $<.032$ & $<.001$ \\
\hline ZK-58-19-805 & 303859097400901 & $03-30-00$ & $<.039$ & $<.11$ & $<.1$ & $<.002$ & E.015 & $<.002$ & $<.043$ & $<.07$ & $<.032$ & $<.001$ \\
\hline ZK-58-27-819 & 303117097421301 & $04-18-00$ & $<.039$ & $<.11$ & $<.1$ & $<.002$ & $<.002$ & $<.002$ & $<.043$ & $<.07$ & $<.032$ & $<.001$ \\
\hline ZP-46-16-102 & 315042103054301 & $01-12-00$ & $<.039$ & $<.11$ & $<.3$ & $<.002$ & $<.002$ & $<.002$ & $<.043$ & $<.07$ & $<.032$ & $<.001$ \\
\hline ZR-19-42-612 & 331902097472301 & 04-04-00 & $<.039$ & $<.11$ & $<.1$ & $<.002$ & $<.002$ & $<.002$ & $<.043$ & $<.07$ & $<.032$ & $<.001$ \\
\hline ZR-19-51-801 & 330906097411701 & 04-04-00 & $<.039$ & $<.11$ & $<.1$ & $<.002$ & $<.002$ & $<.002$ & $<.043$ & $<.07$ & $<.032$ & $<.001$ \\
\hline ZR-19-51-805 & 330839097412401 & 04-04-00 & $<.039$ & $<.11$ & $<.1$ & $<.002$ & $<.002$ & $<.002$ & $<.043$ & $<.07$ & $<.032$ & $<.001$ \\
\hline ZR-19-61-705 & 330014097285401 & $01-25-00$ & $<.039$ & $<.11$ & $<.24$ & $<.002$ & $<.002$ & $<.002$ & $<.043$ & $<.07$ & $<.032$ & $<.001$ \\
\hline ZT-24-50-403 & 331051102511901 & $05-17-00$ & $<.039$ & $<.11$ & $<.1$ & $<.002$ & $<.002$ & $<.002$ & $<.043$ & $<.07$ & $<.032$ & $<.001$ \\
\hline ZT-27-02-103 & 325819102501601 & $05-23-00$ & $<.039$ & $<.11$ & $<.1$ & $<.002$ & $<.002$ & $<.002$ & .060 & $<.07$ & $<.032$ & $<.001$ \\
\hline ZT-27-02-105 & 325819102503701 & $05-23-00$ & $<.039$ & $<.11$ & $<.1$ & $<.002$ & E.013 & $<.002$ & $<.043$ & $<.07$ & $<.032$ & $<.001$ \\
\hline
\end{tabular}


Table 5. Concentrations of soluble pesticides in public water-supply wells sampled in Texas, November 1999-January 2001—Continued

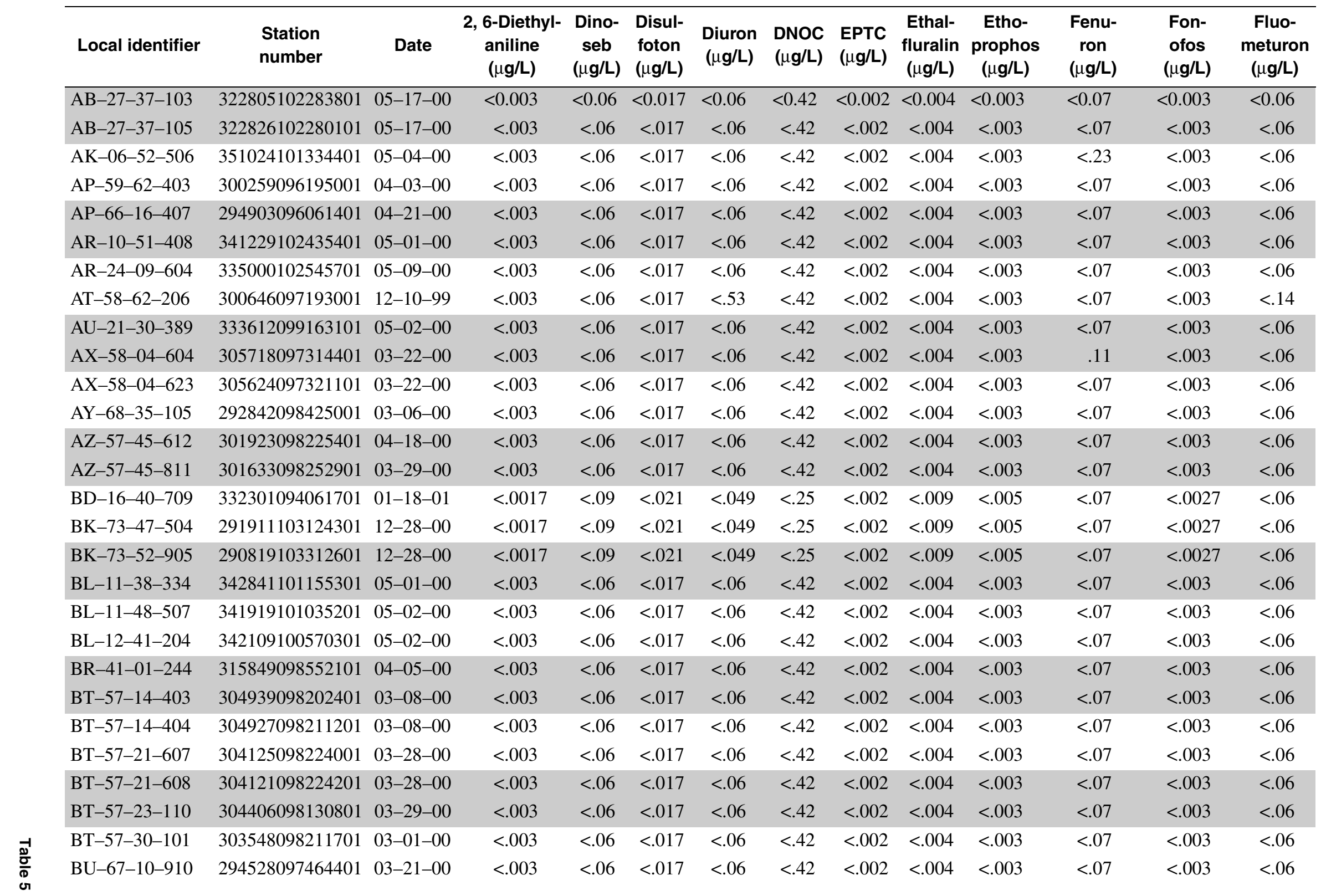


Table 5. Concentrations of soluble pesticides in public water-supply wells sampled in Texas, November 1999-January 2001-Continued

\begin{tabular}{|c|c|c|c|c|c|c|c|c|c|c|c|c|c|}
\hline Local identifier & $\begin{array}{l}\text { Station } \\
\text { number }\end{array}$ & Date & $\begin{array}{c}\text { 2, 6-Diethyl- } \\
\text { aniline } \\
(\mu \mathrm{g} / \mathrm{L})\end{array}$ & $\begin{array}{c}\text { Dino- } \\
\text { seb } \\
(\mu \mathrm{g} / \mathrm{L})\end{array}$ & $\begin{array}{l}\text { Disul- } \\
\text { foton } \\
(\mu \mathrm{g} / \mathrm{L})\end{array}$ & $\begin{array}{l}\text { Diuron } \\
\text { ( } \mu \mathrm{g} / \mathrm{L})\end{array}$ & $\begin{array}{l}\text { DNOC } \\
(\mu \mathrm{g} / \mathrm{L})\end{array}$ & $\begin{array}{l}\text { EPTC } \\
(\mu g / L)\end{array}$ & $\begin{array}{l}\text { Ethal- } \\
\text { fluralin } \\
(\mu \mathrm{g} / \mathrm{L})\end{array}$ & $\begin{array}{l}\text { Etho- } \\
\text { prophos } \\
(\mu \mathrm{g} / \mathrm{L})\end{array}$ & $\begin{array}{c}\text { Fenu- } \\
\text { ron } \\
(\mu \mathrm{g} / \mathrm{L})\end{array}$ & $\begin{array}{l}\text { Fon- } \\
\text { ofos } \\
(\mu \mathrm{g} / \mathrm{L})\end{array}$ & $\begin{array}{c}\text { Fluo- } \\
\text { meturon } \\
(\mu \mathrm{g} / \mathrm{L})\end{array}$ \\
\hline $\mathrm{BX}-30-55-936$ & 320939099093801 & $12-18-00$ & $<0.0017$ & $<0.09$ & $<0.021$ & $<0.056$ & $<0.25$ & $<0.002$ & $<0.009$ & $<0.005$ & $<0.07$ & $<0.0027$ & $<0.06$ \\
\hline DA-06-28-202 & 353704101343701 & $05-03-00$ & $<.003$ & $<.06$ & $<.017$ & $<.06$ & $<.42$ & $<.002$ & $<.004$ & $<.003$ & $<.07$ & $<.003$ & $<.06$ \\
\hline DL-14-62-604 & 340406098160701 & 05-10-00 & $<.003$ & $<.06$ & $<.017$ & $<.06$ & $<.42$ & $<.002$ & $<.004$ & $<.003$ & $<.07$ & $<.003$ & $<.06$ \\
\hline DP-24-18-308 & 334352102450601 & 05-09-00 & $<.003$ & $<.06$ & $<.017$ & $<.06$ & $<.42$ & $<.002$ & $<.004$ & $<.003$ & $<.07$ & $<.003$ & $<.06$ \\
\hline DP-25-24-304 & 334245103011001 & $05-08-00$ & $<.003$ & $<.06$ & $<.017$ & $<.06$ & $<.42$ & $<.002$ & $<.004$ & $<.003$ & $<.07$ & $<.003$ & $<.06$ \\
\hline DU-12-06-609 & 345658100155501 & $05-08-00$ & $<.003$ & $<.06$ & $<.017$ & $<.06$ & $<.42$ & $<.002$ & $<.004$ & $<.003$ & $<.07$ & $<.003$ & $<.06$ \\
\hline DX-68-05-621 & 295520098242301 & $03-22-00$ & $<.003$ & $<.06$ & $<.017$ & $<.06$ & $<.42$ & $<.002$ & $<.004$ & $<.003$ & $<.07$ & $<.003$ & $<.06$ \\
\hline DX-68-06-405 & 295528098222301 & $03-22-00$ & $<.003$ & $<.06$ & $<.017$ & $<.06$ & $<.42$ & $<.002$ & $<.004$ & $<.003$ & $<.07$ & $<.003$ & $<.06$ \\
\hline DY-41-13-307 & 315105098241401 & 04-05-00 & $<.003$ & $<.06$ & $<.017$ & $<.06$ & $<.42$ & $<.002$ & $<.004$ & $<.003$ & $<.07$ & $<.003$ & $<.06$ \\
\hline $\mathrm{HH}-45-35-505$ & 312715102402201 & $01-12-00$ & $<.003$ & $<.06$ & $<.017$ & $<.47$ & $<.42$ & $<.002$ & $<.004$ & $<.003$ & $<.07$ & $<.003$ & $<.06$ \\
\hline HP-12-62-802 & 340210100184901 & 05-05-00 & $<.003$ & $<.06$ & $<.017$ & -- & $<.42$ & $<.002$ & $<.004$ & $<.003$ & -- & $<.003$ & -- \\
\hline HS-28-09-404 & 324810101591201 & $05-16-00$ & $<.003$ & $<.06$ & $<.017$ & $<.06$ & $<.42$ & $<.002$ & $<.004$ & $<.003$ & $<.07$ & $<.003$ & $<.06$ \\
\hline HТ-07-63-204 & 350607102103401 & $05-03-00$ & $<.003$ & $<.06$ & $<.017$ & $<.06$ & $<.42$ & $<.002$ & $<.004$ & $<.003$ & $<.07$ & $<.003$ & $<.06$ \\
\hline HZ-77-34-606 & 282608099455301 & $03-28-00$ & $<.003$ & $<.06$ & $<.017$ & $<.06$ & $<.42$ & $<.002$ & $<.004$ & $<.003$ & $<.07$ & $<.003$ & $<.06$ \\
\hline JA-05-57-613 & 350254100535501 & 05-04-00 & $<.003$ & $<.06$ & $<.017$ & $<.06$ & $<.42$ & $<.002$ & $<.004$ & $<.003$ & $<.07$ & $<.003$ & $<.06$ \\
\hline JA-12-12-404 & 344910100360401 & 05-02-00 & $<.003$ & $<.06$ & $<.017$ & $<.06$ & $<.42$ & $<.002$ & $<.004$ & $<.003$ & $<.07$ & $<.003$ & $<.06$ \\
\hline JH-45-05-924 & 315245102240201 & $05-25-00$ & $<.003$ & $<.06$ & $<.017$ & $<.06$ & $<.42$ & $<.002$ & $<.004$ & $<.003$ & $<.07$ & $<.003$ & $<.06$ \\
\hline JL-49-04-116 & 315757106370201 & 01-06-00 & $<.003$ & $<.06$ & $<.017$ & $<.55$ & $<.42$ & $<.002$ & $<.004$ & $<.003$ & $<.07$ & $<.003$ & $<.06$ \\
\hline JL-49-04-423 & 315708106362301 & 01-03-00 & $<.003$ & $<.06$ & $<.017$ & $<.53$ & $<.42$ & $<.002$ & $<.004$ & $<.003$ & $<.07$ & $<.003$ & $<.06$ \\
\hline JL-49-04-428 & 315517106361401 & 01-06-00 & $<.003$ & $<.06$ & $<.017$ & $<.5$ & $<.42$ & $<.002$ & $<.004$ & $<.003$ & $<.07$ & $<.003$ & $<.06$ \\
\hline JL-49-04-492 & 315623106360601 & $01-06-00$ & $<.003$ & $<.06$ & $<.017$ & $<.52$ & $<.42$ & $<.002$ & $<.004$ & $<.003$ & $<.07$ & $<.003$ & $<.06$ \\
\hline JL-49-22-626 & 314145106163601 & $01-04-00$ & $<.003$ & $<.06$ & $<.017$ & $<.52$ & $<.42$ & $<.002$ & $<.004$ & $<.003$ & $<.07$ & $<.003$ & $<.06$ \\
\hline JL-49-24-423 & 314107106063301 & $01-07-00$ & $<.003$ & $<.06$ & $<.017$ & $<.56$ & $<.42$ & $<.002$ & $<.004$ & $<.003$ & $<.07$ & $<.003$ & $<.06$ \\
\hline JL-49-40-502 & 312648106044701 & 01-04-00 & $<.003$ & $<.06$ & $<.017$ & $<.5$ & $<.42$ & $<.002$ & $<.004$ & $<.003$ & $<.07$ & $<.003$ & $<.06$ \\
\hline$J Y-65-26-520$ & 293314095474702 & 04-03-00 & $<.003$ & $<.06$ & $<.017$ & $<.06$ & $<.42$ & $<.002$ & $<.004$ & $<.003$ & $<.07$ & $<.003$ & $<.06$ \\
\hline KD-27-01-612 & 325523102542701 & $05-22-00$ & $<.003$ & $<.06$ & $<.017$ & $<.06$ & $<.42$ & $<.002$ & $<.004$ & $<.003$ & $<.07$ & $<.003$ & $<.06$ \\
\hline KK-57-42-709 & 301545098502801 & 07-06-00 & $<.003$ & $<.06$ & $<.017$ & $<.06$ & $<.42$ & $<.002$ & $<.004$ & $<.003$ & $<.07$ & $<.003$ & $<.06$ \\
\hline $\mathrm{KK}-57-50-325$ & 301357098471901 & $03-28-00$ & $<.003$ & $<.06$ & $<.017$ & $<.06$ & $<.42$ & $<.002$ & $<.004$ & $<.003$ & $<.07$ & $<.003$ & $<.06$ \\
\hline KK-57-51-303 & 301411098383101 & $03-21-00$ & $<.003$ & $<.06$ & $<.017$ & $<.06$ & $<.42$ & $<.002$ & $<.004$ & $<.003$ & .16 & $<.003$ & $<.06$ \\
\hline $\mathrm{KK}-57-52-107$ & 301430098363401 & $03-21-00$ & $<.003$ & $<.06$ & $<.017$ & $<.06$ & $<.42$ & $<.002$ & $<.004$ & $<.003$ & $<.07$ & $<.003$ & $<.06$ \\
\hline
\end{tabular}


Table 5. Concentrations of soluble pesticides in public water-supply wells sampled in Texas, November 1999-January 2001—Continued

\begin{tabular}{|c|c|c|c|c|c|c|c|c|c|c|c|c|c|}
\hline Local identifier & $\begin{array}{l}\text { Station } \\
\text { number }\end{array}$ & Date & $\begin{array}{c}\text { 2, 6-Diethyl- } \\
\text { aniline } \\
(\mu \mathrm{g} / \mathrm{L})\end{array}$ & $\begin{array}{c}\text { Dino- } \\
\text { seb } \\
(\mu \mathrm{g} / \mathrm{L})\end{array}$ & $\begin{array}{l}\text { Disul- } \\
\text { foton } \\
\text { ( } \mu \mathrm{g} / \mathrm{L})\end{array}$ & $\begin{array}{c}\text { Diuron } \\
(\mu \mathrm{g} / \mathrm{L})\end{array}$ & $\begin{array}{l}\text { DNOC } \\
(\mu \mathbf{g} / L)\end{array}$ & $\begin{array}{l}\text { EPTC } \\
(\mu \mathrm{g} / \mathrm{L})\end{array}$ & $\begin{array}{c}\text { Ethal- } \\
\text { fluralin } \\
(\mu \mathrm{g} / \mathrm{L})\end{array}$ & $\begin{array}{l}\text { Etho- } \\
\text { prophos } \\
(\mu \mathrm{g} / \mathrm{L})\end{array}$ & $\begin{array}{l}\text { Fenu- } \\
\text { ron } \\
(\mu \mathrm{g} / \mathrm{L})\end{array}$ & $\begin{array}{l}\text { Fon- } \\
\text { ofos } \\
(\mu \mathrm{g} / \mathrm{L})\end{array}$ & $\begin{array}{c}\text { Fluo- } \\
\text { meturon } \\
(\mu \mathrm{g} / \mathrm{L})\end{array}$ \\
\hline KP-79-23-101 & 284256097125001 & $02-29-00$ & $<0.003$ & $<0.06$ & $<0.017$ & $<0.06$ & $<0.42$ & $<0.002$ & $<0.004$ & $<0.003$ & $<0.07$ & $<0.003$ & $<0.06$ \\
\hline KS-05-52-105 & 351428100352601 & $05-04-00$ & $<.003$ & $<.06$ & $<.017$ & $<.06$ & $<.42$ & $<.002$ & $<.004$ & $<.003$ & $<.07$ & $<.003$ & $<.06$ \\
\hline KT-18-11-405 & 334844096435701 & 04-19-00 & $<.003$ & $<.06$ & $<.017$ & $<.06$ & $<.42$ & $<.002$ & $<.004$ & $<.003$ & $<.07$ & $<.003$ & $<.06$ \\
\hline KT-18-11-406 & 334840096434001 & 04-19-00 & $<.003$ & $<.06$ & $<.017$ & $<.06$ & $<.42$ & $<.002$ & $<.004$ & $<.003$ & $<.07$ & $<.003$ & $<.06$ \\
\hline KT-18-28-703 & 333039096362901 & 04-19-00 & $<.003$ & $<.06$ & $<.017$ & $<.06$ & $<.42$ & $<.002$ & $<.004$ & $<.003$ & $<.07$ & $<.003$ & $<.06$ \\
\hline KY-11-49-512 & 341116101574601 & 05-04-00 & $<.003$ & $<.06$ & $<.017$ & $<.06$ & $<.42$ & $<.002$ & $<.004$ & $<.003$ & $<.07$ & $<.003$ & $<.06$ \\
\hline KY-11-51-416 & 341032101423801 & $05-04-00$ & $<.003$ & $<.06$ & $<.017$ & $<.06$ & $<.42$ & $<.002$ & $<.004$ & $<.003$ & $<.07$ & $<.003$ & $<.06$ \\
\hline KY-11-57-602 & 340345101531801 & $05-03-00$ & $<.003$ & $<.06$ & $<.017$ & $<.06$ & $<.42$ & $<.002$ & $<.004$ & $<.003$ & $<.07$ & $<.003$ & $<.06$ \\
\hline KZ-12-19-104 & 344234100431601 & 05-09-00 & $<.003$ & $<.06$ & $<.017$ & $<.06$ & $<.42$ & $<.002$ & $<.004$ & $<.003$ & $<.07$ & $<.003$ & $<.06$ \\
\hline LD-13-44-907 & 341523099305701 & $12-19-00$ & $<.0017$ & $<.09$ & $<.021$ & $<.056$ & $<.25$ & $<.002$ & $<.009$ & $<.005$ & $<.07$ & $<.0027$ & $<.06$ \\
\hline LJ-60-64-807 & 300149095045001 & $05-04-00$ & $<.003$ & $<.06$ & $<.017$ & $<.06$ & $<.42$ & $<.002$ & $<.004$ & $<.003$ & $<.07$ & $<.003$ & $<.06$ \\
\hline LP-21-49-317 & 331233099545201 & $05-01-00$ & $<.003$ & $<.06$ & $<.017$ & $<.06$ & $<.42$ & $<.002$ & $<.004$ & $<.003$ & $<.07$ & $<.003$ & $<.06$ \\
\hline LR-67-09-112 & 295137097585801 & $03-21-00$ & $<.003$ & $<.06$ & $<.017$ & $<.06$ & $<.42$ & $<.002$ & $<.004$ & $<.003$ & $<.07$ & $<.003$ & $<.06$ \\
\hline LX-24-15-612 & 334857102092301 & $05-11-00$ & $<.003$ & $<.06$ & $<.017$ & $<.06$ & $<.42$ & $<.002$ & $<.004$ & $<.003$ & $<.07$ & $<.003$ & $<.06$ \\
\hline LX-24-29-306 & 333536102223101 & 05-09-00 & $<.003$ & $<.06$ & $<.017$ & $<.06$ & $<.42$ & $<.002$ & $<.004$ & $<.003$ & $<.07$ & $<.003$ & $<.06$ \\
\hline LX-24-37-405 & 332714102285401 & $05-16-00$ & $<.003$ & $<.06$ & $<.017$ & $<.06$ & $<.42$ & $<.002$ & $<.004$ & $<.003$ & $<.07$ & $<.003$ & $<.06$ \\
\hline LX-24-40-405 & 332624102072901 & $05-17-00$ & $<.003$ & $<.06$ & $<.017$ & $<.06$ & $<.42$ & $<.002$ & $<.004$ & $<.003$ & $<.07$ & $<.003$ & $<.06$ \\
\hline LY-32-26-706 & 323204097500301 & $03-22-00$ & $<.003$ & $<.06$ & $<.017$ & $<.06$ & $<.42$ & $<.002$ & $<.004$ & $<.003$ & $<.07$ & $<.003$ & $<.06$ \\
\hline LY-32-34-208 & 322958097481301 & $03-21-00$ & $<.003$ & $<.06$ & $<.017$ & $<.06$ & $<.42$ & $<.002$ & $<.004$ & $<.003$ & $<.07$ & $<.003$ & $<.06$ \\
\hline LY-32-34-303 & 322928097461701 & 04-03-00 & $<.003$ & $<.06$ & $<.017$ & $<.06$ & $<.42$ & $<.002$ & $<.004$ & $<.003$ & $<.07$ & $<.003$ & $<.06$ \\
\hline LY-32-34-510 & 322537097485701 & $03-21-00$ & $<.003$ & $<.06$ & $<.017$ & $<.06$ & $<.42$ & $<.002$ & $<.004$ & $<.003$ & $<.07$ & $<.003$ & $<.06$ \\
\hline LY-32-34-609 & 322626097454801 & $03-21-00$ & $<.003$ & $<.06$ & $<.017$ & $<.06$ & $<.42$ & $<.002$ & $<.004$ & $<.003$ & $<.07$ & $<.003$ & $<.06$ \\
\hline PB-28-62-105 & 320649101215501 & $04-26-00$ & $<.003$ & $<.06$ & $<.017$ & $<.06$ & $<.42$ & $<.002$ & $<.004$ & $<.003$ & $<.07$ & $<.003$ & $<.06$ \\
\hline PK-43-50-216 & 311422100490201 & $12-20-00$ & $<.0017$ & $<.09$ & $<.021$ & $<.056$ & $<.25$ & $<.002$ & $<.009$ & $<.005$ & $<.07$ & $<.0027$ & $<.06$ \\
\hline PS-52-02-404 & 305502103504101 & 01-10-00 & $<.003$ & $<.06$ & $<.017$ & $<.55$ & $<.42$ & $<.002$ & $<.004$ & $<.003$ & $<.07$ & $<.003$ & $<.06$ \\
\hline PU-84-43-504 & 271841098402801 & 04-04-00 & $<.003$ & $<.06$ & $<.017$ & $<.06$ & $<.42$ & $<.002$ & $<.004$ & $<.003$ & $<.07$ & $<.003$ & $<.06$ \\
\hline PX-32-47-109 & 322201097141601 & 03-20-00 & $<.003$ & $<.06$ & $<.017$ & $<.06$ & $<.42$ & $<.002$ & $<.004$ & $<.003$ & $<.07$ & $<.003$ & $<.06$ \\
\hline PZ-79-10-807 & 284713097493901 & $02-23-00$ & $<.003$ & $<.06$ & $<.017$ & $<.06$ & $<.42$ & $<.002$ & $<.004$ & $<.003$ & $<.07$ & $<.003$ & $<.06$ \\
\hline RS-21-35-103 & 332854099441101 & 05-01-00 & $<.003$ & $<.06$ & $<.017$ & $<.06$ & $<.42$ & $<.002$ & $<.004$ & $<.003$ & $<.07$ & $<.003$ & $<.06$ \\
\hline RU-10-53-312 & 341418102243401 & $05-02-00$ & $<.003$ & $<.06$ & $<.017$ & $<.06$ & $<.42$ & $<.002$ & $<.004$ & $<.003$ & $<.07$ & $<.003$ & $<.06$ \\
\hline
\end{tabular}


Table 5. Concentrations of soluble pesticides in public water-supply wells sampled in Texas, November 1999-January 2001-Continued

\begin{tabular}{|c|c|c|c|c|c|c|c|c|c|c|c|c|c|}
\hline Local identifier & $\begin{array}{l}\text { Station } \\
\text { number }\end{array}$ & Date & $\begin{array}{c}\text { 2, 6-Diethyl- } \\
\text { aniline } \\
(\mu \mathrm{g} / \mathrm{L})\end{array}$ & $\begin{array}{c}\text { Dino- } \\
\text { seb } \\
(\mu \mathrm{g} / \mathrm{L})\end{array}$ & $\begin{array}{l}\text { Disul- } \\
\text { foton } \\
\text { ( } \mu \mathrm{g} / \mathrm{L})\end{array}$ & $\begin{array}{c}\text { Diuron } \\
(\mu \mathrm{g} / L)\end{array}$ & $\begin{array}{l}\text { DNOC } \\
(\mu \mathrm{g} / \mathrm{L})\end{array}$ & $\begin{array}{l}\text { EPTC } \\
(\mu \mathrm{g} / \mathrm{L})\end{array}$ & $\begin{array}{l}\text { Ethal- } \\
\text { fluralin } \\
(\mu \mathrm{g} / \mathrm{L})\end{array}$ & $\begin{array}{l}\text { Etho- } \\
\text { prophos } \\
(\mu \mathrm{g} / \mathrm{L})\end{array}$ & $\begin{array}{l}\text { Fenu- } \\
\text { ron } \\
(\mu \mathrm{g} / \mathrm{L})\end{array}$ & $\begin{array}{l}\text { Fon- } \\
\text { ofos } \\
(\mu \mathrm{g} / \mathrm{L})\end{array}$ & $\begin{array}{c}\text { Fluo- } \\
\text { meturon } \\
(\mu \mathrm{g} / \mathrm{L})\end{array}$ \\
\hline RU-10-60-606 & 340427102302801 & $05-02-00$ & $<0.003$ & $<0.06$ & $<0.017$ & $<0.06$ & $<0.42$ & $<0.002$ & $<0.004$ & $<0.003$ & $<0.07$ & $<0.003$ & $<0.06$ \\
\hline RW-41-63-520 & 310238098104001 & $03-23-00$ & $<.003$ & $<.06$ & $<.017$ & $<.06$ & $<.42$ & $<.002$ & $<.004$ & $<.003$ & $<.07$ & $<.003$ & $<.06$ \\
\hline SK-57-19-201 & 304323098421701 & $03-08-00$ & $<.003$ & $<.06$ & $<.017$ & $<.06$ & $<.42$ & $<.002$ & $<.004$ & $<.003$ & $<.07$ & $<.003$ & $<.06$ \\
\hline SK-57-34-201 & 303001098485201 & 03-09-00 & $<.003$ & $<.13$ & $<.017$ & $<.06$ & $<.42$ & $<.002$ & $<.004$ & $<.003$ & $<.07$ & $<.003$ & $<.06$ \\
\hline SP-23-17-417 & 334043101585701 & $05-10-00$ & $<.003$ & $<.06$ & $<.017$ & $<.06$ & $<.42$ & $<.002$ & $<.004$ & $<.003$ & $<.13$ & $<.003$ & $<.06$ \\
\hline SP-23-18-107 & 334306101504101 & $05-10-00$ & $<.003$ & $<.06$ & $<.017$ & $<.06$ & $<.42$ & $<.002$ & $<.004$ & $<.003$ & $<.07$ & $<.003$ & $<.06$ \\
\hline SP-23-19-812 & 333955101404601 & $05-10-00$ & $<.003$ & $<.06$ & $<.017$ & $<.06$ & $<.42$ & $<.002$ & $<.004$ & $<.003$ & $<.07$ & $<.003$ & $<.06$ \\
\hline SP-24-24-214 & 334419102045201 & $05-18-00$ & $<.003$ & $<.06$ & $<.017$ & $<.06$ & $<.42$ & $<.002$ & $<.004$ & $<.003$ & $<.07$ & $<.003$ & $<.06$ \\
\hline SR-23-41-602 & 331933101543701 & $05-15-00$ & $<.003$ & $<.06$ & $<.017$ & $<.06$ & $<.42$ & $<.002$ & $<.004$ & $<.003$ & $<.07$ & $<.003$ & $<.06$ \\
\hline SS-42-52-504 & 311202099321401 & $03-01-00$ & $<.003$ & $<.06$ & $<.017$ & $<.06$ & $<.42$ & $<.002$ & $<.004$ & $<.003$ & $<.12$ & $<.003$ & $<.06$ \\
\hline ST-40-39-304 & 312745097083301 & $03-23-00$ & $<.003$ & $<.06$ & $<.017$ & $<.06$ & $<.42$ & $<.002$ & $<.004$ & $<.003$ & $<.07$ & $<.003$ & $<.06$ \\
\hline SY-27-39-505 & 322555102105501 & $05-24-00$ & $<.003$ & $<.06$ & $<.017$ & $<.06$ & $<.42$ & $<.002$ & $<.004$ & $<.003$ & $<.07$ & $<.003$ & $<.06$ \\
\hline SY $-28-42-803$ & 321619101495001 & $05-23-00$ & $<.003$ & $<.06$ & $<.017$ & $<.06$ & $<.42$ & $<.002$ & $<.004$ & $<.003$ & $<.07$ & $<.003$ & $<.06$ \\
\hline SY-28-50-908 & 320816101474601 & $05-23-00$ & $<.003$ & $<.06$ & $<.017$ & $<.06$ & $<.42$ & $<.002$ & $<.004$ & $<.003$ & $<.07$ & $<.003$ & $<.06$ \\
\hline TB-76-03-605 & 285657100373801 & $03-28-00$ & $<.003$ & $<.06$ & $<.017$ & $<.06$ & $<.42$ & $<.002$ & $<.004$ & $<.003$ & $<.07$ & $<.003$ & $<.06$ \\
\hline TJ-27-63-712 & 320143102123101 & $12-20-00$ & $<.0017$ & $<.09$ & $<.021$ & $<.056$ & $<.25$ & $<.002$ & $<.009$ & $<.005$ & $<.07$ & $<.0027$ & $<.06$ \\
\hline TJ-28-57-702 & 320221101594901 & $05-25-00$ & $<.003$ & $<.06$ & $<.017$ & $<.06$ & $<.42$ & $<.002$ & $<.004$ & $<.003$ & $<.15$ & $<.003$ & $<.06$ \\
\hline TJ-28-57-903 & 320010101523701 & $05-23-00$ & $<.003$ & $<.06$ & $<.017$ & $<.06$ & $<.42$ & $<.002$ & $<.004$ & $<.003$ & $<.07$ & $<.003$ & $<.06$ \\
\hline TJ-45-07-407 & 315643102131101 & $05-24-00$ & $<.003$ & $<.06$ & $<.017$ & $<.06$ & $<.42$ & $<.002$ & $<.004$ & $<.003$ & $<.07$ & $<.003$ & $<.06$ \\
\hline TJ-45-07-606 & 315702102075401 & $05-25-00$ & $<.003$ & $<.06$ & $<.017$ & $<.06$ & $<.42$ & $<.002$ & $<.004$ & $<.003$ & $<.07$ & $<.003$ & $<.06$ \\
\hline TS-60-36-510 & 302701095331201 & 04-20-00 & $<.003$ & $<.06$ & $<.017$ & $<.06$ & $<.42$ & $<.002$ & $<.004$ & $<.003$ & $<.07$ & $<.003$ & $<.06$ \\
\hline TW-22-02-714 & 335416100502101 & 05-05-00 & $<.003$ & $<.06$ & $<.017$ & $<.06$ & $<.42$ & $<.002$ & $<.004$ & $<.003$ & $<.07$ & $<.003$ & $<.06$ \\
\hline TW-22-02-716 & 335449100513101 & $12-19-00$ & $<.0017$ & $<.09$ & $<.021$ & $<.056$ & $<.25$ & $<.002$ & $<.009$ & $<.005$ & $<.07$ & $<.0027$ & $<.06$ \\
\hline UJ-62-49-712 & 300906093585201 & $04-28-00$ & $<.003$ & $<.06$ & $<.017$ & $<.06$ & $<.42$ & $<.002$ & $<.004$ & $<.003$ & $<.07$ & $<.003$ & $<.06$ \\
\hline UK-31-05-501 & 325545098255501 & $03-22-00$ & $<.003$ & $<.06$ & $<.017$ & $<.06$ & $<.42$ & $<.002$ & $<.004$ & $<.003$ & $<.07$ & $<.003$ & $<.06$ \\
\hline UK-31-24-501 & 324210098034701 & $03-22-00$ & $<.003$ & $<.06$ & $<.017$ & $<.06$ & $<.42$ & $<.002$ & $<.004$ & $<.003$ & $<.07$ & $<.003$ & $<.06$ \\
\hline UL-35-48-202 & 322207094034501 & $04-25-00$ & $<.003$ & $<.06$ & $<.017$ & $<.06$ & $<.42$ & $<.002$ & $<.004$ & $<.003$ & $<.07$ & $<.003$ & $<.06$ \\
\hline UP-32-10-604 & 324926097455001 & 02-09-00 & $<.003$ & $<.06$ & $<.017$ & $<.49$ & $<.42$ & $<.002$ & $<.004$ & $<.003$ & $<.07$ & $<.003$ & $<.06$ \\
\hline UP-32-11-103 & 325005097440101 & 02-09-00 & $<.003$ & $<.06$ & $<.017$ & $<.57$ & $<.42$ & $<.002$ & $<.004$ & $<.003$ & $<.07$ & $<.003$ & $<.06$ \\
\hline UP-32-11-709 & 324649097442001 & 02-09-00 & $<.003$ & $<.06$ & $<.017$ & $<.57$ & $<.42$ & $<.002$ & $<.004$ & $<.003$ & $<.07$ & $<.003$ & $<.06$ \\
\hline
\end{tabular}


Table 5. Concentrations of soluble pesticides in public water-supply wells sampled in Texas, November 1999-January 2001—Continued

\begin{tabular}{|c|c|c|c|c|c|c|c|c|c|c|c|c|c|}
\hline Local identifier & $\begin{array}{l}\text { Station } \\
\text { number }\end{array}$ & Date & $\begin{array}{c}\text { 2, 6-Diethyl- } \\
\text { aniline } \\
(\mu \mathrm{g} / \mathrm{L})\end{array}$ & $\begin{array}{c}\text { Dino- } \\
\text { seb } \\
(\mu \mathrm{g} / \mathrm{L})\end{array}$ & $\begin{array}{l}\text { Disul- } \\
\text { foton } \\
\text { ( } \mu \mathrm{g} / \mathrm{L})\end{array}$ & $\begin{array}{c}\text { Diuron } \\
(\mu \mathrm{g} / \mathrm{L})\end{array}$ & $\begin{array}{l}\text { DNOC } \\
(\mu \mathbf{g} / L)\end{array}$ & $\begin{array}{l}\text { EPTC } \\
(\mu \mathrm{g} / \mathrm{L})\end{array}$ & $\begin{array}{l}\text { Ethal- } \\
\text { fluralin } \\
(\mu \mathrm{g} / \mathrm{L})\end{array}$ & $\begin{array}{l}\text { Etho- } \\
\text { prophos } \\
(\mu \mathrm{g} / \mathrm{L})\end{array}$ & $\begin{array}{l}\text { Fenu- } \\
\text { ron } \\
(\mu \mathrm{g} / \mathrm{L})\end{array}$ & $\begin{array}{l}\text { Fon- } \\
\text { ofos } \\
(\mu \mathrm{g} / \mathrm{L})\end{array}$ & $\begin{array}{c}\text { Fluo- } \\
\text { meturon } \\
(\mu \mathrm{g} / \mathrm{L})\end{array}$ \\
\hline UP-32-11-801 & 324548097411701 & $02-10-00$ & $<0.003$ & $<0.06$ & $<0.017$ & $<0.56$ & $<0.42$ & $<0.002$ & $<0.004$ & $<0.003$ & $<0.07$ & $<0.003$ & $<0.06$ \\
\hline UP-32-25-602 & 323439097524301 & 02-09-00 & $<.003$ & $<.06$ & $<.017$ & $<.59$ & $<.42$ & $<.002$ & $<.004$ & $<.003$ & $<.07$ & $<.003$ & $<.16$ \\
\hline UP-32-28-403 & 323356097361801 & 04-10-00 & $<.003$ & $<.06$ & $<.017$ & $<.06$ & $<.42$ & $<.002$ & $<.004$ & $<.003$ & $<.07$ & $<.003$ & $<.06$ \\
\hline US-53-08-601 & 305723102015801 & $03-14-00$ & $<.003$ & $<.06$ & $<.017$ & $<.06$ & $<.42$ & $<.002$ & $<.004$ & $<.003$ & $<.07$ & $<.003$ & $<.06$ \\
\hline US-54-18-503 & 304131101492401 & $03-14-00$ & $<.003$ & $<.06$ & $<.017$ & $<.06$ & $<.42$ & $<.002$ & $<.004$ & $<.003$ & $<.07$ & $<.003$ & $<.06$ \\
\hline UT-60-24-110 & 304251095060001 & $04-26-00$ & $<.003$ & $<.06$ & $<.017$ & $<.06$ & $<.42$ & $<.002$ & $<.004$ & $<.003$ & $<.07$ & $<.003$ & $<.06$ \\
\hline UY-07-64-914 & 350224102020301 & $05-03-00$ & $<.003$ & $<.06$ & $<.017$ & $<.06$ & $<.42$ & $<.002$ & $<.004$ & $<.003$ & $<.07$ & $<.003$ & $<.06$ \\
\hline UZ-44-37-505 & 312656101265101 & $01-13-00$ & $<.003$ & $<.06$ & $<.017$ & $<.5$ & $<.42$ & $<.002$ & $<.004$ & $<.003$ & $<.07$ & $<.003$ & $<.06$ \\
\hline WB-16-18-704 & 333942094503001 & $04-26-00$ & $<.003$ & $<.06$ & $<.017$ & $<.06$ & $<.42$ & $<.002$ & $<.004$ & $<.003$ & $<.07$ & $<.003$ & $<.06$ \\
\hline WD-46-46-210 & 312041103174201 & $01-11-00$ & $<.003$ & $<.06$ & $<.017$ & $<.43$ & $<.42$ & $<.002$ & $<.004$ & $<.003$ & $<.07$ & $<.003$ & $<.15$ \\
\hline WK-39-51-801 & 310956096402901 & $04-20-00$ & $<.003$ & $<.06$ & $<.017$ & $<.06$ & $<.42$ & $<.002$ & $<.004$ & $<.003$ & $<.07$ & $<.003$ & $<.06$ \\
\hline WP-43-31-211 & 313551100110801 & $04-25-00$ & $<.003$ & $<.06$ & $<.017$ & $<.06$ & $<.42$ & $<.002$ & $<.004$ & $<.003$ & $<.07$ & $<.003$ & $<.06$ \\
\hline WT-37-31-706 & 313110094131201 & $04-27-00$ & $<.003$ & $<.06$ & $<.017$ & $<.06$ & $<.42$ & $<.002$ & $<.004$ & $<.003$ & $<.07$ & $<.003$ & $<.06$ \\
\hline XH-34-37-312 & 322832095245401 & $04-25-00$ & $<.003$ & $<.06$ & $<.017$ & $<.06$ & $<.42$ & $<.002$ & $<.004$ & $<.003$ & $<.07$ & $<.003$ & $<.06$ \\
\hline XP-43-09-119 & 315033100584001 & $04-26-00$ & $<.003$ & $<.06$ & $<.017$ & $<.06$ & $<.42$ & $<.002$ & $<.004$ & $<.003$ & $<.07$ & $<.003$ & $<.06$ \\
\hline $\mathrm{XR}-22-54-201$ & 331310100180801 & $05-01-00$ & $<.003$ & $<.06$ & $<.017$ & $<.06$ & $<.42$ & $<.002$ & $<.004$ & $<.003$ & $<.07$ & $<.003$ & $<.06$ \\
\hline XT-11-26-923 & 343046101463701 & $05-01-00$ & $<.003$ & $<.06$ & $<.017$ & $<.06$ & $<.42$ & $<.002$ & $<.004$ & $<.003$ & $<.07$ & $<.003$ & $<.06$ \\
\hline XU-32-04-604 & 325525097304801 & $01-05-00$ & $<.003$ & $<.06$ & $<.017$ & $<.55$ & $<.42$ & $<.002$ & $<.004$ & $<.003$ & $<.07$ & $<.003$ & $<.06$ \\
\hline XU-32-04-605 & 325525097304802 & $01-05-00$ & $<.003$ & $<.06$ & $<.017$ & $<.49$ & $<.42$ & $<.002$ & $<.004$ & $<.003$ & $<.07$ & $<.003$ & $<.06$ \\
\hline XU-32-05-805 & 325335097272401 & $04-03-00$ & $<.003$ & $<.06$ & $<.017$ & $<.06$ & $<.42$ & $<.002$ & $<.004$ & $<.003$ & $<.07$ & $<.003$ & $<.06$ \\
\hline XU-32-12-307 & 325101097313101 & $03-23-00$ & $<.003$ & $<.06$ & $<.017$ & $<.06$ & $<.42$ & $<.002$ & $<.004$ & $<.003$ & $<.07$ & $<.003$ & $<.06$ \\
\hline XU-32-13-405 & 324901097284701 & $01-24-00$ & $<.003$ & $<.06$ & $<.017$ & $<.64$ & $<.42$ & $<.002$ & $<.004$ & $<.003$ & $<.07$ & $<.003$ & $<.06$ \\
\hline XU-32-13-503 & 324816097272401 & $01-24-00$ & $<.003$ & $<.06$ & $<.017$ & $<.56$ & $<.42$ & $<.002$ & $<.004$ & $<.003$ & $<.07$ & $<.003$ & $<.15$ \\
\hline XU-32-23-701 & 323911097131001 & $03-20-00$ & $<.003$ & $<.06$ & $<.017$ & $<.06$ & $<.42$ & $<.002$ & $<.004$ & $<.003$ & $<.07$ & $<.003$ & $<.06$ \\
\hline XY-24-54-606 & 331112102160201 & $05-17-00$ & $<.003$ & $<.06$ & $<.017$ & $<.06$ & $<.42$ & $<.002$ & $<.004$ & $<.003$ & $<.07$ & $<.003$ & $<.06$ \\
\hline YB-43-19-701 & 313943100425301 & $01-14-00$ & $<.003$ & $<.06$ & $<.017$ & $<.56$ & $<.42$ & $<.002$ & $<.004$ & $<.003$ & $<.07$ & $<.003$ & $<.06$ \\
\hline YB-43-27-201 & 313638100400201 & $01-14-00$ & $<.003$ & $<.06$ & $<.017$ & $<.48$ & $<.42$ & $<.002$ & $<.004$ & $<.003$ & $<.07$ & $<.003$ & $<.06$ \\
\hline YB-43-44-102 & 312003100370101 & $01-14-00$ & $<.003$ & $<.06$ & $<.017$ & $<.51$ & $<.42$ & $<.002$ & $<.004$ & $<.003$ & $<.07$ & $<.003$ & $<.06$ \\
\hline YD-57-40-703 & 302306098050701 & 04-19-00 & $<.003$ & $<.06$ & $<.017$ & $<.06$ & $<.42$ & $<.002$ & $<.004$ & $<.003$ & $<.07$ & $<.003$ & $<.06$ \\
\hline YD-58-50-830 & 300938097490601 & $11-03-99$ & $<.003$ & $<.06$ & $<.017$ & $<.06$ & $<.42$ & $<.002$ & $<.004$ & $<.003$ & $<.07$ & $<.003$ & $<.06$ \\
\hline
\end{tabular}


Table 5. Concentrations of soluble pesticides in public water-supply wells sampled in Texas, November 1999-January 2001-Continued

\begin{tabular}{|c|c|c|c|c|c|c|c|c|c|c|c|c|c|}
\hline Local identifier & $\begin{array}{l}\text { Station } \\
\text { number }\end{array}$ & Date & $\begin{array}{c}\text { 2, 6-Diethyl- } \\
\text { aniline } \\
(\mu \mathrm{g} / \mathrm{L})\end{array}$ & $\begin{array}{c}\text { Dino- } \\
\text { seb } \\
(\mu \mathrm{g} / \mathrm{L})\end{array}$ & $\begin{array}{l}\text { Disul- } \\
\text { foton } \\
(\mu \mathrm{g} / \mathrm{L})\end{array}$ & $\begin{array}{c}\text { Diuron } \\
\text { ( } \mu \mathrm{g} / \mathrm{L})\end{array}$ & $\begin{array}{l}\text { DNOC } \\
(\mu \mathrm{g} / \mathrm{L})\end{array}$ & $\begin{array}{l}\text { EPTC } \\
(\mu g / L)\end{array}$ & $\begin{array}{c}\text { Ethal- } \\
\text { fluralin } \\
(\mu \mathrm{g} / \mathrm{L})\end{array}$ & $\begin{array}{c}\text { Etho- } \\
\text { prophos } \\
(\mu \mathrm{g} / \mathrm{L})\end{array}$ & $\begin{array}{l}\text { Fenu- } \\
\text { ron } \\
(\mu \mathrm{g} / \mathrm{L})\end{array}$ & $\begin{array}{l}\text { Fon- } \\
\text { ofos } \\
(\mu \mathrm{g} / L)\end{array}$ & $\begin{array}{c}\text { Fluo- } \\
\text { meturon } \\
(\mu \mathrm{g} / \mathrm{L})\end{array}$ \\
\hline YD-58-52-304 & 301305097314601 & $04-27-00$ & $<0.003$ & $<0.06$ & $<0.017$ & $<0.06$ & $<0.42$ & $<0.002$ & $<0.004$ & $<0.003$ & $<0.07$ & $<0.003$ & $<0.06$ \\
\hline YH-60-13-308 & 305125095225701 & 01-19-01 & $<.0017$ & $<.2$ & $<.021$ & $<.049$ & $<.25$ & $<.002$ & $<.009$ & $<.005$ & $<.07$ & $<.0027$ & $<.06$ \\
\hline YP-69-27-107 & 293652099442501 & $10-19-00$ & $<.0017$ & $<.09$ & $<.021$ & $<.049$ & $<.25$ & $<.002$ & $<.009$ & $<.005$ & $<.07$ & $<.0027$ & $<.06$ \\
\hline YP-69-27-402 & 293450099433701 & $10-19-00$ & $<.0017$ & $<.09$ & $<.021$ & $<.049$ & $<.25$ & $<.002$ & $<.009$ & $<.005$ & $<.07$ & $<.0027$ & $<.06$ \\
\hline YP-69-27-704 & 293037099430301 & $10-18-00$ & $<.0017$ & $<.09$ & $<.021$ & $<.049$ & $<.25$ & $<.002$ & $<.009$ & $<.005$ & $<.07$ & $<.0027$ & $<.06$ \\
\hline YP-69-28-303 & 293644099314601 & $10-18-00$ & $<.0017$ & $<.09$ & $<.021$ & $<.049$ & $<.25$ & $<.002$ & $<.009$ & $<.005$ & $<.07$ & $<.0027$ & $<.06$ \\
\hline YT-79-24-101 & 284431097071801 & $02-29-00$ & $<.003$ & $<.06$ & $<.017$ & $<.06$ & $<.42$ & $<.002$ & $<.004$ & $<.003$ & $<.07$ & $<.003$ & $<.06$ \\
\hline$Y X-46-32-625$ & 313252103010301 & $01-11-00$ & $<.003$ & $<.06$ & $<.017$ & $<.49$ & $<.42$ & $<.002$ & $<.004$ & $<.003$ & $<.07$ & $<.003$ & $<.06$ \\
\hline$Y X-46-40-311$ & 312848103012901 & $01-11-00$ & $<.003$ & $<.06$ & $<.017$ & $<.83$ & $<.42$ & $<.002$ & $<.004$ & $<.003$ & $<.07$ & $<.003$ & $<.06$ \\
\hline YY-59-54-902 & 300824096152400 & 04-19-00 & $<.003$ & $<.06$ & $<.017$ & $<.06$ & $<.42$ & $<.002$ & $<.004$ & $<.003$ & $<.07$ & $<.003$ & $<.06$ \\
\hline ZB-05-29-819 & 353215100261201 & $05-10-00$ & $<.003$ & $<.06$ & $<.017$ & $<.06$ & $<.42$ & $<.002$ & $<.004$ & $<.003$ & $<.07$ & $<.003$ & $<.06$ \\
\hline ZB-05-38-512 & 352709100182901 & $05-08-00$ & $<.003$ & $<.06$ & $<.017$ & $<.06$ & $<.42$ & $<.002$ & $<.004$ & $<.003$ & $<.07$ & $<.003$ & $<.06$ \\
\hline $\mathrm{ZH}-13-46-414$ & 341848099222501 & $05-09-00$ & $<.003$ & $<.06$ & $<.017$ & $<.06$ & $<.42$ & $<.002$ & $<.004$ & $<.003$ & $<.07$ & $<.003$ & $<.06$ \\
\hline $\mathrm{ZH}-13-61-620$ & 340451099224401 & $12-19-00$ & $<.0017$ & $<.09$ & $<.021$ & $<.056$ & $<.25$ & $<.002$ & $<.009$ & $<.005$ & $<.07$ & $<.0027$ & $<.06$ \\
\hline $\mathrm{ZK}-58-19-620$ & 304151097390301 & $03-30-00$ & $<.003$ & $<.06$ & $<.017$ & .02 & $<.42$ & $<.002$ & $<.004$ & $<.003$ & $<.07$ & $<.003$ & $<.06$ \\
\hline ZK-58-19-804 & 303809097404701 & $03-30-00$ & $<.003$ & $<.06$ & $<.017$ & $<.06$ & $<.42$ & $<.002$ & $<.004$ & $<.003$ & $<.07$ & $<.003$ & $<.06$ \\
\hline ZK-58-19-805 & 303859097400901 & $03-30-00$ & $<.003$ & $<.06$ & $<.017$ & $<.06$ & $<.42$ & $<.002$ & $<.004$ & $<.003$ & $<.07$ & $<.003$ & $<.06$ \\
\hline $\mathrm{ZK}-58-27-819$ & 303117097421301 & $04-18-00$ & $<.003$ & $<.06$ & $<.017$ & $<.06$ & $<.42$ & $<.002$ & $<.004$ & $<.003$ & $<.07$ & $<.003$ & $<.06$ \\
\hline ZP-46-16-102 & 315042103054301 & $01-12-00$ & $<.003$ & $<.06$ & $<.017$ & $<.49$ & $<.42$ & $<.002$ & $<.004$ & $<.003$ & E.02 & $<.003$ & $<.06$ \\
\hline ZR-19-42-612 & 331902097472301 & 04-04-00 & $<.003$ & $<.06$ & $<.017$ & $<.06$ & $<.42$ & $<.002$ & $<.004$ & $<.003$ & $<.07$ & $<.003$ & $<.06$ \\
\hline ZR-19-51-801 & 330906097411701 & 04-04-00 & $<.003$ & $<.06$ & $<.017$ & $<.06$ & $<.42$ & $<.002$ & $<.004$ & $<.003$ & $<.07$ & $<.003$ & $<.06$ \\
\hline ZR-19-51-805 & 330839097412401 & 04-04-00 & $<.003$ & $<.06$ & $<.017$ & $<.06$ & $<.42$ & $<.002$ & $<.004$ & $<.003$ & $<.07$ & $<.003$ & $<.06$ \\
\hline ZR-19-61-705 & 330014097285401 & $01-25-00$ & $<.003$ & $<.06$ & $<.017$ & $<.06$ & $<.42$ & $<.002$ & $<.004$ & $<.003$ & $<.07$ & $<.003$ & $<.21$ \\
\hline ZT-24-50-403 & 331051102511901 & $05-17-00$ & $<.003$ & $<.06$ & $<.017$ & $<.06$ & $<.42$ & $<.002$ & $<.004$ & $<.003$ & $<.07$ & $<.003$ & $<.06$ \\
\hline ZT-27-02-103 & 325819102501601 & $05-23-00$ & $<.003$ & $<.06$ & $<.017$ & $<.06$ & $<.42$ & $<.002$ & $<.004$ & $<.003$ & $<.07$ & $<.003$ & $<.06$ \\
\hline ZT-27-02-105 & 325819102503701 & $05-23-00$ & $<.003$ & $<.06$ & $<.017$ & .14 & $<.42$ & $<.002$ & $<.004$ & $<.003$ & $<.07$ & $<.003$ & $<.06$ \\
\hline
\end{tabular}


Table 5. Concentrations of soluble pesticides in public water-supply wells sampled in Texas, November 1999-January 2001—Continued

\begin{tabular}{|c|c|c|c|c|c|c|c|c|c|c|c|c|}
\hline Local identifier & $\begin{array}{l}\text { Station } \\
\text { number }\end{array}$ & Date & $\begin{array}{c}\text { 3-Hydroxy- } \\
\text { carbofuran } \\
(\mu \mathrm{g} / \mathrm{L})\end{array}$ & $\begin{array}{c}\text { Lindane } \\
(\mu \mathrm{g} / \mathrm{L})\end{array}$ & $\begin{array}{c}\text { Linuron } \\
(\mu \mathrm{g} / \mathrm{L})\end{array}$ & $\begin{array}{c}\text { Malathion } \\
(\mu \mathrm{g} / \mathrm{L})\end{array}$ & $\begin{array}{l}\text { MCPA } \\
(\mu \mathrm{g} / \mathrm{L})\end{array}$ & $\begin{array}{l}\text { MCPB } \\
(\mu \mathrm{g} / \mathrm{L})\end{array}$ & $\begin{array}{l}\text { Methio- } \\
\text { carb } \\
(\mu \mathrm{g} / \mathrm{L})\end{array}$ & $\begin{array}{l}\text { Metho- } \\
\text { myl } \\
(\mu \mathrm{g} / \mathrm{L})\end{array}$ & $\begin{array}{l}\text { Metola- } \\
\text { chlor } \\
(\mu \mathrm{g} / \mathrm{L})\end{array}$ & $\begin{array}{l}\text { Metri- } \\
\text { buzin } \\
\text { ( } \mu \text { g/L) }\end{array}$ \\
\hline AB-27-37-103 & 322805102283801 & $05-17-00$ & $<0.11$ & $<0.004$ & $<0.002$ & $<0.005$ & $<0.17$ & $<0.13$ & $<0.026$ & $<0.13$ & $<0.002$ & $<0.004$ \\
\hline AB-27-37-105 & 322826102280101 & $05-17-00$ & $<.11$ & $<.004$ & $<.002$ & $<.005$ & $<.17$ & $<.13$ & $<.026$ & $<.017$ & $<.002$ & $<.004$ \\
\hline AK-06-52-506 & 351024101334401 & 05-04-00 & $<.17$ & $<.004$ & $<.002$ & $<.005$ & $<.17$ & $<.13$ & $<.026$ & $<.32$ & $<.002$ & $<.004$ \\
\hline AP-59-62-403 & 300259096195001 & 04-03-00 & $<.11$ & $<.004$ & $<.002$ & $<.005$ & $<.17$ & $<.13$ & $<.026$ & $<.017$ & $<.002$ & $<.004$ \\
\hline AP-66-16-407 & 294903096061401 & $04-21-00$ & $<.11$ & $<.004$ & $<.002$ & $<.005$ & $<.17$ & $<.13$ & $<.026$ & $<.017$ & $<.002$ & $<.004$ \\
\hline AR-10-51-408 & 341229102435401 & $05-01-00$ & $<.11$ & $<.004$ & $<.002$ & $<.005$ & $<.17$ & $<.13$ & $<.026$ & $<.017$ & $<.002$ & $<.004$ \\
\hline AR-24-09-604 & 335000102545701 & 05-09-00 & $<.18$ & $<.004$ & $<.002$ & $<.005$ & $<.17$ & $<.13$ & $<.026$ & $<.017$ & $<.002$ & $<.004$ \\
\hline AT-58-62-206 & 300646097193001 & $12-10-99$ & $<.11$ & $<.004$ & $<.002$ & $<.005$ & $<.17$ & $<.13$ & $<.026$ & $<.017$ & $<.002$ & $<.3$ \\
\hline AU-21-30-389 & 333612099163101 & $05-02-00$ & $<.11$ & $<.004$ & $<.002$ & $<.005$ & $<.17$ & $<.13$ & $<.026$ & $<.017$ & $<.002$ & $<.004$ \\
\hline$A X-58-04-604$ & 305718097314401 & $03-22-00$ & $<.11$ & $<.004$ & $<.002$ & $<.005$ & $<.17$ & $<.13$ & $<.026$ & $<.11$ & E.004 & $<.004$ \\
\hline $\mathrm{AX}-58-04-623$ & 305624097321101 & $03-22-00$ & $<.11$ & $<.004$ & $<.002$ & $<.005$ & $<.17$ & $<.13$ & $<.026$ & $<.017$ & $<.002$ & $<.004$ \\
\hline AY-68-35-105 & 292842098425001 & $03-06-00$ & $<.11$ & $<.004$ & $<.002$ & $<.005$ & $<.17$ & $<.13$ & $<.026$ & $<.017$ & $<.002$ & $<.004$ \\
\hline AZ-57-45-612 & 301923098225401 & $04-18-00$ & $<.11$ & $<.004$ & $<.002$ & $<.005$ & $<.17$ & $<.13$ & $<.026$ & $<.017$ & $<.002$ & $<.004$ \\
\hline AZ-57-45-811 & 301633098252901 & 03-29-00 & $<.11$ & $<.004$ & $<.002$ & $<.005$ & $<.17$ & $<.13$ & $<.026$ & $<.017$ & $<.002$ & $<.004$ \\
\hline BD-16-40-709 & 332301094061701 & $01-18-01$ & $<.011$ & $<.004$ & $<.035$ & $<.027$ & $<.08$ & $<.13$ & $<.07$ & $<.017$ & $<.013$ & $<.006$ \\
\hline BK-73-47-504 & 291911103124301 & $12-28-00$ & $<.011$ & $<.004$ & $<.035$ & $<.027$ & $<.08$ & $<.13$ & $<.07$ & $<.017$ & $<.013$ & $<.006$ \\
\hline BK-73-52-905 & 290819103312601 & $12-28-00$ & $<.011$ & $<.004$ & $<.035$ & $<.027$ & $<.08$ & $<.13$ & $<.07$ & $<.017$ & $<.013$ & $<.006$ \\
\hline BL-11-38-334 & 342841101155301 & $05-01-00$ & $<.11$ & $<.004$ & $<.002$ & $<.005$ & $<.17$ & $<.13$ & $<.026$ & $<.017$ & $<.002$ & $<.004$ \\
\hline BL-11-48-507 & 341919101035201 & $05-02-00$ & $<.11$ & $<.004$ & $<.002$ & $<.005$ & $<.17$ & $<.13$ & $<.026$ & $<.017$ & $<.002$ & $<.004$ \\
\hline BL-12-41-204 & 342109100570301 & $05-02-00$ & $<.11$ & $<.004$ & $<.002$ & $<.005$ & $<.17$ & $<.13$ & $<.026$ & $<.017$ & $<.002$ & $<.004$ \\
\hline BR-41-01-244 & 315849098552101 & 04-05-00 & $<.11$ & $<.004$ & $<.002$ & $<.005$ & $<.17$ & $<.13$ & $<.026$ & $<.017$ & $<.002$ & $<.004$ \\
\hline BT-57-14-403 & 304939098202401 & $03-08-00$ & $<.11$ & $<.004$ & $<.002$ & $<.005$ & $<.17$ & $<.13$ & $<.026$ & $<.017$ & $<.002$ & $<.004$ \\
\hline BT-57-14-404 & 304927098211201 & 03-08-00 & $<.11$ & $<.004$ & $<.002$ & $<.005$ & $<.17$ & $<.13$ & $<.026$ & $<.54$ & $<.002$ & $<.004$ \\
\hline BT-57-21-607 & 304125098224001 & $03-28-00$ & $<.11$ & $<.004$ & $<.002$ & $<.005$ & $<.17$ & $<.13$ & $<.026$ & $<.017$ & $<.002$ & $<.004$ \\
\hline BT-57-21-608 & 304121098224201 & $03-28-00$ & $<.11$ & $<.004$ & $<.002$ & $<.005$ & $<.17$ & $<.13$ & $<.026$ & $<.017$ & $<.002$ & $<.004$ \\
\hline BT-57-23-110 & 304406098130801 & 03-29-00 & $<.11$ & $<.004$ & $<.002$ & $<.005$ & $<.17$ & $<.13$ & $<.026$ & $<.017$ & $<.002$ & $<.004$ \\
\hline BT-57-30-101 & 303548098211701 & $03-01-00$ & $<.11$ & $<.004$ & $<.002$ & $<.005$ & $<.17$ & $<.13$ & $<.026$ & $<1.02$ & $<.002$ & $<.004$ \\
\hline BU-67-10-910 & 294528097464401 & $03-21-00$ & $<.11$ & $<.004$ & $<.002$ & $<.005$ & $<.17$ & $<.13$ & $<.026$ & $<.017$ & $<.002$ & $<.004$ \\
\hline
\end{tabular}


Table 5. Concentrations of soluble pesticides in public water-supply wells sampled in Texas, November 1999-January 2001-Continued

\begin{tabular}{|c|c|c|c|c|c|c|c|c|c|c|c|c|}
\hline Local identifier & $\begin{array}{l}\text { Station } \\
\text { number }\end{array}$ & Date & $\begin{array}{l}\text { 3-Hydroxy- } \\
\text { carbofuran } \\
(\mu \mathrm{g} / \mathrm{L})\end{array}$ & $\begin{array}{c}\text { Lindane } \\
\text { ( } \mu \mathrm{g} / \mathrm{L})\end{array}$ & $\begin{array}{l}\text { Linuron } \\
\text { ( } \mu \mathrm{g} / \mathrm{L})\end{array}$ & $\begin{array}{c}\text { Malathion } \\
\quad(\mu \mathrm{g} / \mathrm{L})\end{array}$ & $\begin{array}{l}\text { MCPA } \\
(\mu \mathrm{g} / \mathrm{L})\end{array}$ & $\begin{array}{l}\text { MCPB } \\
(\mu \mathrm{g} / \mathrm{L})\end{array}$ & $\begin{array}{c}\text { Methio- } \\
\text { carb } \\
(\mu \mathrm{g} / \mathrm{L})\end{array}$ & $\begin{array}{c}\text { Metho- } \\
\text { myl } \\
(\mu \mathrm{g} / \mathrm{L})\end{array}$ & $\begin{array}{c}\text { Metola- } \\
\text { chlor } \\
(\mu \mathrm{g} / \mathrm{L})\end{array}$ & $\begin{array}{l}\text { Metri- } \\
\text { buzin } \\
(\mu \mathrm{g} / \mathrm{L})\end{array}$ \\
\hline $\mathrm{BX}-30-55-936$ & 320939099093801 & $12-18-00$ & $<0.11$ & $<0.004$ & $<0.035$ & $<0.027$ & $<0.08$ & $<0.13$ & $<0.07$ & $<0.017$ & $<0.013$ & $<0.006$ \\
\hline DA-06-28-202 & 353704101343701 & $05-03-00$ & $<.11$ & $<.004$ & $<.002$ & $<.005$ & $<.17$ & $<.13$ & $<.026$ & $<.017$ & $<.002$ & $<.004$ \\
\hline DL-14-62-604 & 340406098160701 & $05-10-00$ & $<.11$ & $<.004$ & $<.002$ & $<.005$ & $<.17$ & $<.13$ & $<.026$ & $<.017$ & $<.002$ & $<.004$ \\
\hline DP-24-18-308 & 334352102450601 & 05-09-00 & $<.11$ & $<.004$ & $<.002$ & $<.005$ & $<.17$ & $<.13$ & $<.026$ & $<.017$ & $<.002$ & $<.004$ \\
\hline DP-25-24-304 & 334245103011001 & $05-08-00$ & $<.11$ & $<.004$ & $<.002$ & $<.005$ & $<.17$ & $<.13$ & $<.026$ & $<.017$ & $<.002$ & $<.004$ \\
\hline DU-12-06-609 & 345658100155501 & $05-08-00$ & $<.11$ & $<.004$ & $<.002$ & $<.005$ & $<.17$ & $<.13$ & $<.026$ & $<.017$ & $<.002$ & $<.004$ \\
\hline DX-68-05-621 & 295520098242301 & $03-22-00$ & $<.11$ & $<.004$ & $<.002$ & $<.005$ & $<.17$ & $<.13$ & $<.026$ & $<.98$ & $<.002$ & $<.004$ \\
\hline DX-68-06-405 & 295528098222301 & $03-22-00$ & $<.11$ & $<.004$ & $<.002$ & $<.005$ & $<.17$ & $<.13$ & $<.026$ & $<.017$ & $<.002$ & $<.004$ \\
\hline DY-41-13-307 & 315105098241401 & $04-05-00$ & $<.11$ & $<.004$ & $<.002$ & $<.005$ & $<.17$ & $<.13$ & $<.026$ & $<.017$ & E.001 & $<.004$ \\
\hline $\mathrm{HH}-45-35-505$ & 312715102402201 & $01-12-00$ & $<.11$ & $<.004$ & $<.002$ & $<.005$ & $<.17$ & $<.13$ & $<.026$ & $<.017$ & $<.002$ & $<.004$ \\
\hline HP-12-62-802 & 340210100184901 & 05-05-00 & -- & $<.004$ & $<.002$ & $<.005$ & $<.17$ & $<.13$ & -- & -- & $<.002$ & $<.004$ \\
\hline HS-28-09-404 & 324810101591201 & $05-16-00$ & $<.11$ & $<.004$ & $<.002$ & $<.005$ & $<.17$ & $<.13$ & $<.026$ & $<.26$ & $<.002$ & $<.004$ \\
\hline HТ-07-63-204 & 350607102103401 & $05-03-00$ & $<.11$ & $<.004$ & $<.002$ & $<.005$ & $<.17$ & $<.13$ & $<.026$ & $<.017$ & $<.002$ & $<.004$ \\
\hline HZ-77-34-606 & 282608099455301 & $03-28-00$ & $<.11$ & $<.004$ & $<.002$ & $<.005$ & $<.17$ & $<.13$ & $<.026$ & $<.68$ & $<.002$ & $<.004$ \\
\hline JA-05-57-613 & 350254100535501 & 05-04-00 & $<.11$ & $<.004$ & $<.002$ & $<.005$ & $<.17$ & $<.13$ & $<.026$ & $<.017$ & $<.002$ & $<.004$ \\
\hline JA-12-12-404 & 344910100360401 & $05-02-00$ & $<.11$ & $<.004$ & $<.002$ & $<.005$ & $<.17$ & $<.13$ & $<.026$ & $<.017$ & $<.002$ & $<.004$ \\
\hline JH-45-05-924 & 315245102240201 & $05-25-00$ & $<.11$ & $<.004$ & $<.002$ & $<.005$ & $<.17$ & $<.13$ & $<.026$ & $<.017$ & $<.002$ & $<.004$ \\
\hline JL-49-04-116 & 315757106370201 & $01-06-00$ & $<.11$ & $<.004$ & $<.002$ & $<.005$ & $<.17$ & $<.13$ & $<.026$ & $<.017$ & $<.002$ & $<.004$ \\
\hline JL-49-04-423 & 315708106362301 & $01-03-00$ & $<.11$ & $<.004$ & $<.002$ & $<.005$ & $<.17$ & $<.13$ & $<.026$ & $<.017$ & $<.002$ & $<.004$ \\
\hline JL-49-04-428 & 315517106361401 & $01-06-00$ & $<.11$ & $<.004$ & $<.002$ & $<.005$ & $<.17$ & $<.13$ & $<.026$ & $<.017$ & $<.002$ & $<.004$ \\
\hline JL-49-04-492 & 315623106360601 & $01-06-00$ & $<.11$ & $<.004$ & $<.002$ & $<.005$ & $<.17$ & $<.13$ & $<.026$ & $<.017$ & $<.002$ & $<.004$ \\
\hline $\mathrm{JL}-49-22-626$ & 314145106163601 & $01-04-00$ & $<.11$ & $<.004$ & $<.002$ & $<.005$ & $<.17$ & $<.13$ & $<.026$ & $<.017$ & $<.002$ & $<.004$ \\
\hline JL-49-24-423 & 314107106063301 & $01-07-00$ & $<.11$ & $<.004$ & $<.002$ & $<.005$ & $<.17$ & $<.13$ & $<.026$ & $<.017$ & $<.002$ & $<.004$ \\
\hline JL-49-40-502 & 312648106044701 & $01-04-00$ & $<.11$ & $<.004$ & $<.002$ & $<.005$ & $<.17$ & $<.13$ & $<.026$ & $<.017$ & $<.002$ & $<.004$ \\
\hline$J Y-65-26-520$ & 293314095474702 & 04-03-00 & $<.11$ & $<.004$ & $<.002$ & $<.005$ & $<.17$ & $<.13$ & $<.026$ & $<.017$ & $<.002$ & $<.004$ \\
\hline KD-27-01-612 & 325523102542701 & $05-22-00$ & $<.11$ & $<.004$ & $<.002$ & $<.005$ & $<.17$ & $<.13$ & $<.026$ & $<.017$ & $<.002$ & $<.004$ \\
\hline KK-57-42-709 & 301545098502801 & 07-06-00 & $<.11$ & $<.004$ & $<.002$ & $<.005$ & $<.17$ & $<.13$ & $<.026$ & $<.017$ & $<.002$ & $<.004$ \\
\hline $\mathrm{KK}-57-50-325$ & 301357098471901 & $03-28-00$ & $<.11$ & $<.004$ & $<.002$ & $<.005$ & $<.17$ & $<.13$ & $<.026$ & $<.017$ & $<.002$ & $<.004$ \\
\hline KK-57-51-303 & 301411098383101 & $03-21-00$ & $<.11$ & $<.004$ & $<.002$ & $<.005$ & $<.17$ & $<.13$ & $<.026$ & $<.017$ & $<.002$ & $<.004$ \\
\hline $\mathrm{KK}-57-52-107$ & 301430098363401 & $03-21-00$ & $<.11$ & $<.004$ & $<.002$ & $<.005$ & $<.17$ & $<.13$ & $<.026$ & $<.017$ & $<.002$ & $<.004$ \\
\hline
\end{tabular}


Table 5. Concentrations of soluble pesticides in public water-supply wells sampled in Texas, November 1999-January 2001-Continued

\begin{tabular}{|c|c|c|c|c|c|c|c|c|c|c|c|c|}
\hline Local identifier & $\begin{array}{l}\text { Station } \\
\text { number }\end{array}$ & Date & $\begin{array}{c}\text { 3-Hydroxy- } \\
\text { carbofuran } \\
(\mu \mathrm{g} / \mathrm{L})\end{array}$ & $\begin{array}{l}\text { Lindane } \\
\text { ( } \mu \mathrm{g} / \mathrm{L})\end{array}$ & $\begin{array}{l}\text { Linuron } \\
(\mu \mathrm{g} / \mathrm{L})\end{array}$ & $\begin{array}{l}\text { Malathion } \\
(\mu \mathrm{g} / \mathrm{L})\end{array}$ & $\begin{array}{l}\text { MCPA } \\
(\mu \mathrm{g} / \mathrm{L})\end{array}$ & $\begin{array}{l}\text { MCPB } \\
(\mu \mathrm{g} / \mathrm{L})\end{array}$ & $\begin{array}{l}\text { Methio- } \\
\text { carb } \\
(\mu \mathrm{g} / \mathrm{L})\end{array}$ & $\begin{array}{l}\text { Metho- } \\
\text { myl } \\
(\mu \mathrm{g} / \mathrm{L})\end{array}$ & $\begin{array}{l}\text { Metola- } \\
\text { chlor } \\
(\mu \mathrm{g} / \mathrm{L})\end{array}$ & $\begin{array}{l}\text { Metri- } \\
\text { buzin } \\
(\mu g / L)\end{array}$ \\
\hline KP-79-23-101 & 284256097125001 & $02-29-00$ & $<0.11$ & $<0.004$ & $<0.002$ & $<0.005$ & $<0.17$ & $<0.13$ & $<0.026$ & $<0.017$ & $<0.002$ & $<0.004$ \\
\hline KS-05-52-105 & 351428100352601 & $05-04-00$ & $<.11$ & $<.004$ & $<.002$ & $<.005$ & $<.17$ & $<.13$ & $<.026$ & $<.13$ & $<.002$ & $<.004$ \\
\hline KT-18-11-405 & 334844096435701 & 04-19-00 & $<.11$ & $<.004$ & $<.002$ & $<.005$ & $<.17$ & $<.13$ & $<.026$ & $<.017$ & $<.002$ & $<.004$ \\
\hline KT-18-11-406 & 334840096434001 & 04-19-00 & $<.11$ & $<.004$ & $<.002$ & $<.005$ & $<.17$ & $<.13$ & $<.026$ & $<.017$ & $<.002$ & $<.004$ \\
\hline KT-18-28-703 & 333039096362901 & 04-19-00 & $<.11$ & $<.004$ & $<.002$ & $<.005$ & $<.17$ & $<.13$ & $<.026$ & $<.017$ & $<.002$ & $<.004$ \\
\hline KY-11-49-512 & 341116101574601 & $05-04-00$ & $<.11$ & $<.004$ & $<.002$ & $<.005$ & $<.17$ & $<.13$ & $<.026$ & $<.017$ & $<.002$ & $<.004$ \\
\hline $\mathrm{KY}-11-51-416$ & 341032101423801 & 05-04-00 & $<.11$ & $<.004$ & $<.002$ & $<.005$ & $<.17$ & $<.13$ & $<.026$ & $<.2$ & $<.002$ & $<.004$ \\
\hline KY-11-57-602 & 340345101531801 & $05-03-00$ & $<.11$ & $<.004$ & $<.002$ & $<.005$ & $<.17$ & $<.13$ & $<.1$ & $<.017$ & $<.002$ & $<.004$ \\
\hline KZ-12-19-104 & 344234100431601 & 05-09-00 & $<.11$ & $<.004$ & $<.002$ & $<.005$ & $<.17$ & $<.13$ & $<.026$ & $<.017$ & $<.002$ & $<.004$ \\
\hline LD-13-44-907 & 341523099305701 & $12-19-00$ & $<.11$ & $<.004$ & $<.035$ & $<.027$ & $<.08$ & $<.13$ & $<.07$ & $<.017$ & $<.013$ & $<.006$ \\
\hline LJ-60-64-807 & 300149095045001 & $05-04-00$ & $<.11$ & $<.004$ & $<.002$ & $<.005$ & $<.17$ & $<.13$ & $<.026$ & $<.017$ & $<.002$ & $<.004$ \\
\hline LP-21-49-317 & 331233099545201 & $05-01-00$ & $<.11$ & $<.004$ & $<.002$ & $<.005$ & $<.17$ & $<.13$ & $<.026$ & $<.017$ & $<.002$ & $<.004$ \\
\hline LR-67-09-112 & 295137097585801 & $03-21-00$ & $<.11$ & $<.004$ & $<.002$ & $<.005$ & $<.17$ & $<.13$ & $<.026$ & $<.017$ & $<.002$ & $<.004$ \\
\hline LX-24-15-612 & 334857102092301 & $05-11-00$ & $<.11$ & $<.004$ & $<.002$ & $<.005$ & $<.17$ & $<.13$ & $<.026$ & $<.017$ & $<.002$ & $<.004$ \\
\hline LX-24-29-306 & 333536102223101 & 05-09-00 & $<.11$ & $<.004$ & $<.002$ & $<.005$ & $<.17$ & $<.13$ & $<.026$ & $<.017$ & $<.002$ & $<.004$ \\
\hline LX-24-37-405 & 332714102285401 & $05-16-00$ & $<.11$ & $<.004$ & $<.002$ & $<.005$ & $<.17$ & $<.13$ & $<.026$ & $<.017$ & $<.002$ & $<.004$ \\
\hline LX-24-40-405 & 332624102072901 & $05-17-00$ & $<.11$ & $<.004$ & $<.002$ & $<.005$ & $<.17$ & $<.13$ & $<.026$ & $<.17$ & $<.002$ & $<.004$ \\
\hline LY-32-26-706 & 323204097500301 & $03-22-00$ & $<.11$ & $<.004$ & $<.002$ & $<.005$ & $<.17$ & $<.13$ & $<.026$ & $<.32$ & $<.002$ & $<.004$ \\
\hline LY-32-34-208 & 322958097481301 & $03-21-00$ & $<.11$ & $<.004$ & $<.002$ & $<.005$ & $<.17$ & $<.13$ & $<.026$ & $<.017$ & $<.002$ & $<.004$ \\
\hline LY-32-34-303 & 322928097461701 & 04-03-00 & $<.11$ & $<.004$ & $<.002$ & $<.005$ & $<.17$ & $<.13$ & $<.026$ & $<.017$ & $<.002$ & $<.004$ \\
\hline LY-32-34-510 & 322537097485701 & $03-21-00$ & $<.11$ & $<.004$ & $<.002$ & $<.005$ & $<.17$ & $<.13$ & $<.026$ & $<.017$ & $<.002$ & $<.004$ \\
\hline LY-32-34-609 & 322626097454801 & $03-21-00$ & $<.11$ & $<.004$ & $<.002$ & $<.005$ & $<.17$ & $<.13$ & $<.026$ & $<.017$ & $<.002$ & $<.004$ \\
\hline PB-28-62-105 & 320649101215501 & 04-26-00 & $<.11$ & $<.004$ & $<.002$ & $<.005$ & $<.17$ & $<.13$ & $<.026$ & $<.017$ & $<.002$ & $<.004$ \\
\hline$P K-43-50-216$ & 311422100490201 & $12-20-00$ & $<.11$ & $<.004$ & $<.035$ & $<.027$ & $<.08$ & $<.13$ & $<.07$ & $<.103$ & $<.013$ & $<.006$ \\
\hline PS-52-02-404 & 305502103504101 & 01-10-00 & $<.11$ & $<.004$ & $<.002$ & $<.005$ & $<.17$ & $<.13$ & $<.026$ & $<.017$ & $<.002$ & $<.004$ \\
\hline PU-84-43-504 & 271841098402801 & 04-04-00 & $<.11$ & $<.004$ & $<.002$ & $<.005$ & $<.17$ & $<.13$ & $<.026$ & $<.017$ & $<.002$ & $<.004$ \\
\hline PX-32-47-109 & 322201097141601 & $03-20-00$ & $<.11$ & $<.004$ & $<.002$ & $<.005$ & $<.17$ & $<.13$ & $<.026$ & $<.017$ & $<.002$ & $<.004$ \\
\hline PZ-79-10-807 & 284713097493901 & $02-23-00$ & $<.11$ & $<.004$ & $<.002$ & $<.005$ & $<.17$ & $<.13$ & $<.026$ & $<.017$ & $<.002$ & $<.004$ \\
\hline RS-21-35-103 & 332854099441101 & $05-01-00$ & $<.11$ & $<.004$ & $<.002$ & $<.005$ & $<.17$ & $<.13$ & $<.026$ & $<.017$ & $<.002$ & $<.004$ \\
\hline RU-10-53-312 & 341418102243401 & $05-02-00$ & $<.11$ & $<.004$ & $<.002$ & $<.005$ & $<.17$ & $<.13$ & $<.026$ & $<.017$ & .005 & $<.004$ \\
\hline
\end{tabular}


Table 5. Concentrations of soluble pesticides in public water-supply wells sampled in Texas, November 1999-January 2001-Continued

\begin{tabular}{|c|c|c|c|c|c|c|c|c|c|c|c|c|}
\hline Local identifier & $\begin{array}{l}\text { Station } \\
\text { number }\end{array}$ & Date & $\begin{array}{l}\text { 3-Hydroxy- } \\
\text { carbofuran } \\
(\mu \mathrm{g} / \mathrm{L})\end{array}$ & $\begin{array}{c}\text { Lindane } \\
\text { ( } \mu \mathrm{g} / \mathrm{L})\end{array}$ & $\begin{array}{l}\text { Linuron } \\
\text { ( } \mu \mathrm{g} / \mathrm{L})\end{array}$ & $\begin{array}{c}\text { Malathion } \\
\quad(\mu \mathrm{g} / \mathrm{L})\end{array}$ & $\begin{array}{l}\text { MCPA } \\
(\mu \mathrm{g} / \mathrm{L})\end{array}$ & $\begin{array}{l}\text { MCPB } \\
(\mu \mathrm{g} / \mathrm{L})\end{array}$ & $\begin{array}{c}\text { Methio- } \\
\text { carb } \\
(\mu \mathrm{g} / \mathrm{L})\end{array}$ & $\begin{array}{l}\text { Metho- } \\
\text { myl } \\
(\mu \mathrm{g} / \mathrm{L})\end{array}$ & $\begin{array}{c}\text { Metola- } \\
\text { chlor } \\
(\mu \mathrm{g} / \mathrm{L})\end{array}$ & $\begin{array}{l}\text { Metri- } \\
\text { buzin } \\
(\mu \mathrm{g} / \mathrm{L})\end{array}$ \\
\hline RU-10-60-606 & 340427102302801 & $05-02-00$ & $<0.11$ & $<0.004$ & $<0.002$ & $<0.005$ & $<0.17$ & $<0.13$ & $<0.026$ & $<0.017$ & $<0.002$ & $<0.004$ \\
\hline$R W-41-63-520$ & 310238098104001 & $03-23-00$ & $<.11$ & $<.004$ & $<.002$ & $<.005$ & $<.17$ & $<.13$ & $<.026$ & $<.017$ & $<.002$ & $<.004$ \\
\hline SK-57-19-201 & 304323098421701 & 03-08-00 & $<.11$ & $<.004$ & $<.002$ & $<.005$ & $<.17$ & $<.13$ & $<.026$ & $<.017$ & $<.002$ & $<.004$ \\
\hline SK-57-34-201 & 303001098485201 & 03-09-00 & $<.11$ & $<.004$ & $<.002$ & $<.005$ & $<.17$ & $<.13$ & $<.026$ & $<.017$ & $<.002$ & $<.004$ \\
\hline SP-23-17-417 & 334043101585701 & $05-10-00$ & $<.12$ & $<.004$ & $<.002$ & $<.005$ & $<.17$ & $<.13$ & $<.026$ & $<.017$ & $<.002$ & $<.004$ \\
\hline SP-23-18-107 & 334306101504101 & $05-10-00$ & $<.11$ & $<.004$ & $<.002$ & $<.005$ & $<.17$ & $<.17$ & $<.026$ & $<.017$ & $<.002$ & $<.004$ \\
\hline SP-23-19-812 & 333955101404601 & 05-10-00 & $<.11$ & $<.004$ & $<.002$ & $<.005$ & $<.17$ & $<.13$ & $<.026$ & $<.017$ & $<.002$ & $<.004$ \\
\hline SP-24-24-214 & 334419102045201 & $05-18-00$ & $<.11$ & $<.004$ & $<.002$ & $<.005$ & $<.17$ & $<.13$ & $<.026$ & $<.017$ & $<.002$ & $<.004$ \\
\hline SR-23-41-602 & 331933101543701 & $05-15-00$ & $<.11$ & $<.004$ & $<.002$ & $<.005$ & $<.17$ & $<.16$ & $<.026$ & $<15.93$ & $<.002$ & $<.004$ \\
\hline SS-42-52-504 & 311202099321401 & $03-01-00$ & $<.11$ & $<.004$ & $<.002$ & $<.005$ & $<.17$ & $<.13$ & $<.026$ & $<.017$ & $<.002$ & $<.004$ \\
\hline ST-40-39-304 & 312745097083301 & $03-23-00$ & $<.11$ & $<.004$ & $<.002$ & $<.005$ & $<.17$ & $<.13$ & $<.026$ & $<.56$ & $<.002$ & $<.004$ \\
\hline SY-27-39-505 & 322555102105501 & $05-24-00$ & $<.11$ & $<.004$ & $<.002$ & $<.005$ & $<.17$ & $<.13$ & $<.026$ & $<.017$ & $<.002$ & $<.004$ \\
\hline$S Y-28-42-803$ & 321619101495001 & $05-23-00$ & $<.11$ & $<.004$ & $<.002$ & $<.005$ & $<.17$ & $<.13$ & $<.026$ & $<.017$ & $<.002$ & $<.004$ \\
\hline SY-28-50-908 & 320816101474601 & $05-23-00$ & $<.11$ & $<.004$ & $<.002$ & $<.005$ & $<.17$ & $<.13$ & $<.026$ & $<.017$ & $<.002$ & $<.004$ \\
\hline TB-76-03-605 & 285657100373801 & $03-28-00$ & $<.11$ & $<.004$ & $<.002$ & $<.005$ & $<.17$ & $<.13$ & $<.026$ & $<.017$ & $<.002$ & $<.004$ \\
\hline TJ-27-63-712 & 320143102123101 & $12-20-00$ & $<.11$ & $<.004$ & $<.035$ & $<.027$ & $<.08$ & $<.13$ & $<.07$ & $<.12$ & $<.013$ & $<.006$ \\
\hline TJ-28-57-702 & 320221101594901 & $05-25-00$ & $<.11$ & $<.004$ & $<.002$ & $<.005$ & $<.17$ & $<.13$ & $<.07$ & $<.017$ & $<.002$ & $<.004$ \\
\hline TJ-28-57-903 & 320010101523701 & $05-23-00$ & $<.11$ & $<.004$ & $<.002$ & $<.005$ & $<.17$ & $<.13$ & $<.026$ & $<.017$ & $<.002$ & $<.004$ \\
\hline TJ-45-07-407 & 315643102131101 & $05-24-00$ & $<.11$ & $<.004$ & $<.002$ & $<.005$ & $<.17$ & $<.13$ & $<.026$ & $<.017$ & $<.002$ & $<.004$ \\
\hline TJ-45-07-606 & 315702102075401 & $05-25-00$ & $<.11$ & $<.004$ & $<.002$ & $<.005$ & $<.17$ & $<.13$ & $<.026$ & $<.017$ & $<.002$ & $<.004$ \\
\hline TS-60-36-510 & 302701095331201 & $04-20-00$ & $<.11$ & $<.004$ & $<.002$ & $<.005$ & $<.17$ & $<.13$ & $<.026$ & $<.017$ & $<.002$ & $<.004$ \\
\hline TW-22-02-714 & 335416100502101 & $05-05-00$ & $<.11$ & $<.004$ & $<.002$ & $<.005$ & $<.17$ & $<.13$ & $<.026$ & $<.017$ & $<.002$ & $<.004$ \\
\hline TW-22-02-716 & 335449100513101 & $12-19-00$ & $<.11$ & $<.004$ & $<.035$ & $<.027$ & $<.08$ & $<.13$ & $<.07$ & $<.017$ & $<.013$ & $<.006$ \\
\hline UJ-62-49-712 & 300906093585201 & 04-28-00 & $<.11$ & $<.004$ & $<.002$ & $<.005$ & $<.17$ & $<.13$ & $<.026$ & $<.017$ & $<.002$ & $<.004$ \\
\hline $\mathrm{UK}-31-05-501$ & 325545098255501 & $03-22-00$ & $<.11$ & $<.004$ & $<.002$ & $<.005$ & $<.17$ & $<.13$ & $<.026$ & $<.017$ & $<.002$ & $<.004$ \\
\hline UK-31-24-501 & 324210098034701 & $03-22-00$ & $<.11$ & $<.004$ & $<.002$ & $<.005$ & $<.17$ & $<.13$ & $<.026$ & $<.017$ & $<.002$ & $<.004$ \\
\hline UL-35-48-202 & 322207094034501 & $04-25-00$ & $<.11$ & $<.004$ & $<.002$ & $<.005$ & $<.17$ & $<.13$ & $<.026$ & $<.017$ & $<.002$ & $<.004$ \\
\hline UP-32-10-604 & 324926097455001 & 02-09-00 & $<.11$ & $<.004$ & $<.002$ & $<.005$ & $<.17$ & $<.13$ & $<.026$ & $<.017$ & $<.002$ & $<.004$ \\
\hline UP-32-11-103 & 325005097440101 & 02-09-00 & $<.11$ & $<.004$ & $<.002$ & $<.005$ & $<.17$ & $<.13$ & $<.026$ & $<.017$ & $<.002$ & $<.004$ \\
\hline UP-32-11-709 & 324649097442001 & 02-09-00 & $<.11$ & $<.004$ & $<.002$ & $<.005$ & $<.17$ & $<.13$ & $<.026$ & $<.017$ & $<.002$ & $<.004$ \\
\hline
\end{tabular}


Table 5. Concentrations of soluble pesticides in public water-supply wells sampled in Texas, November 1999-January 2001—Continued

\begin{tabular}{|c|c|c|c|c|c|c|c|c|c|c|c|c|}
\hline Local identifier & $\begin{array}{l}\text { Station } \\
\text { number }\end{array}$ & Date & $\begin{array}{c}\text { 3-Hydroxy- } \\
\text { carbofuran } \\
(\mu \mathrm{g} / \mathrm{L})\end{array}$ & $\begin{array}{c}\text { Lindane } \\
(\mu \mathrm{g} / \mathrm{L})\end{array}$ & $\begin{array}{c}\text { Linuron } \\
(\mu \mathrm{g} / \mathrm{L})\end{array}$ & $\begin{array}{c}\text { Malathion } \\
(\mu \mathrm{g} / \mathrm{L})\end{array}$ & $\begin{array}{l}\text { MCPA } \\
(\mu \mathbf{g} / \mathrm{L})\end{array}$ & $\begin{array}{l}\text { MCPB } \\
(\mu \mathrm{g} / \mathrm{L})\end{array}$ & $\begin{array}{c}\text { Methio- } \\
\text { carb } \\
(\mu \mathrm{g} / \mathrm{L})\end{array}$ & $\begin{array}{c}\text { Metho- } \\
\text { myl } \\
(\mu \mathrm{g} / \mathrm{L})\end{array}$ & $\begin{array}{l}\text { Metola- } \\
\text { chlor } \\
(\mu \mathrm{g} / \mathrm{L})\end{array}$ & $\begin{array}{l}\text { Metri- } \\
\text { buzin } \\
(\mu g / L)\end{array}$ \\
\hline UP-32-11-801 & 324548097411701 & $02-10-00$ & $<0.11$ & $<0.004$ & $<0.002$ & $<0.005$ & $<0.17$ & $<0.13$ & $<0.026$ & $<0.017$ & $<0.002$ & $<0.004$ \\
\hline UP-32-25-602 & 323439097524301 & 02-09-00 & $<.11$ & $<.004$ & $<.002$ & $<.005$ & $<.17$ & $<.13$ & $<.026$ & $<.98$ & $<.002$ & $<.004$ \\
\hline UP-32-28-403 & 323356097361801 & 04-10-00 & $<.11$ & $<.004$ & $<.002$ & $<.005$ & $<.17$ & $<.13$ & $<.026$ & $<.017$ & $<.002$ & $<.004$ \\
\hline US-53-08-601 & 305723102015801 & $03-14-00$ & $<.11$ & $<.004$ & $<.002$ & $<.005$ & $<.17$ & $<.13$ & $<.026$ & $<.017$ & $<.002$ & $<.004$ \\
\hline US-54-18-503 & 304131101492401 & $03-14-00$ & $<.11$ & $<.004$ & $<.002$ & $<.005$ & $<.17$ & $<.13$ & $<.026$ & $<.017$ & $<.002$ & $<.03$ \\
\hline UT-60-24-110 & 304251095060001 & $04-26-00$ & $<.11$ & $<.004$ & $<.002$ & $<.005$ & $<.17$ & $<.13$ & $<.026$ & $<.017$ & $<.002$ & $<.004$ \\
\hline UY-07-64-914 & 350224102020301 & $05-03-00$ & $<.11$ & $<.004$ & $<.002$ & $<.005$ & $<.17$ & $<.13$ & $<.026$ & $<.017$ & $<.002$ & $<.004$ \\
\hline UZ-44-37-505 & 312656101265101 & $01-13-00$ & $<.11$ & $<.004$ & $<.002$ & $<.005$ & $<.17$ & $<.13$ & $<.026$ & $<.017$ & $<.002$ & $<.004$ \\
\hline WB-16-18-704 & 333942094503001 & 04-26-00 & $<.11$ & $<.004$ & $<.002$ & $<.005$ & $<.17$ & $<.13$ & $<.026$ & $<.017$ & $<.002$ & $<.004$ \\
\hline WD-46-46-210 & 312041103174201 & $01-11-00$ & $<.11$ & $<.004$ & $<.002$ & $<.005$ & $<.17$ & $<.13$ & $<.026$ & $<.017$ & $<.002$ & $<.004$ \\
\hline WK-39-51-801 & 310956096402901 & 04-20-00 & $<.11$ & $<.004$ & $<.002$ & $<.005$ & $<.17$ & $<.13$ & $<.026$ & $<.017$ & $<.002$ & $<.004$ \\
\hline WP-43-31-211 & 313551100110801 & $04-25-00$ & $<.11$ & $<.004$ & $<.002$ & $<.005$ & $<.17$ & $<.13$ & $<.026$ & $<.017$ & $<.002$ & $<.004$ \\
\hline WT-37-31-706 & 313110094131201 & $04-27-00$ & $<.11$ & $<.004$ & $<.002$ & $<.005$ & $<.17$ & $<.13$ & $<.026$ & $<.017$ & $<.002$ & $<.004$ \\
\hline XH-34-37-312 & 322832095245401 & $04-25-00$ & $<.11$ & $<.004$ & $<.002$ & $<.005$ & $<.17$ & $<.13$ & $<.026$ & $<.017$ & $<.002$ & $<.004$ \\
\hline XP-43-09-119 & 315033100584001 & $04-26-00$ & $<.11$ & $<.004$ & $<.002$ & $<.005$ & $<.17$ & $<.13$ & $<.026$ & $<.017$ & $<.002$ & $<.004$ \\
\hline XR-22-54-201 & 331310100180801 & $05-01-00$ & $<.11$ & $<.004$ & $<.002$ & $<.005$ & $<.17$ & $<.13$ & $<.026$ & $<.017$ & $<.002$ & $<.004$ \\
\hline XT-11-26-923 & 343046101463701 & $05-01-00$ & $<.11$ & $<.004$ & $<.002$ & $<.005$ & $<.17$ & $<.13$ & $<.026$ & $<.017$ & $<.002$ & $<.004$ \\
\hline XU-32-04-604 & 325525097304801 & $01-05-00$ & $<.11$ & $<.004$ & $<.002$ & $<.005$ & $<.17$ & $<.13$ & $<.026$ & $<.017$ & $<.002$ & $<.004$ \\
\hline XU-32-04-605 & 325525097304802 & $01-05-00$ & $<.11$ & $<.004$ & $<.002$ & $<.005$ & $<.17$ & $<.13$ & $<.026$ & $<.017$ & $<.002$ & $<.004$ \\
\hline XU-32-05-805 & 325335097272401 & 04-03-00 & $<.11$ & $<.004$ & $<.002$ & $<.005$ & $<.17$ & $<.13$ & $<.026$ & $<.017$ & $<.002$ & $<.004$ \\
\hline XU-32-12-307 & 325101097313101 & $03-23-00$ & $<.11$ & $<.004$ & $<.002$ & $<.005$ & $<.17$ & $<.13$ & $<.026$ & $<.017$ & $<.002$ & $<.004$ \\
\hline XU-32-13-405 & 324901097284701 & $01-24-00$ & $<.11$ & $<.004$ & $<.002$ & $<.005$ & $<.17$ & $<.13$ & $<.026$ & $<.17$ & $<.002$ & $<.004$ \\
\hline XU-32-13-503 & 324816097272401 & $01-24-00$ & $<.11$ & $<.004$ & $<.002$ & $<.005$ & $<.17$ & $<.13$ & $<.026$ & $<.017$ & $<.002$ & $<.004$ \\
\hline XU-32-23-701 & 323911097131001 & $03-20-00$ & $<.11$ & $<.004$ & $<.002$ & $<.005$ & $<.17$ & $<.13$ & $<.026$ & $<.017$ & $<.002$ & $<.004$ \\
\hline XY-24-54-606 & 331112102160201 & $05-17-00$ & $<.11$ & $<.004$ & $<.002$ & $<.005$ & $<.17$ & $<.13$ & $<.026$ & $<.14$ & $<.002$ & $<.004$ \\
\hline YB-43-19-701 & 313943100425301 & $01-14-00$ & $<.11$ & $<.004$ & $<.002$ & $<.005$ & $<.17$ & $<.13$ & $<.026$ & $<.23$ & $<.002$ & $<.004$ \\
\hline YB-43-27-201 & 313638100400201 & 01-14-00 & $<.11$ & $<.004$ & $<.002$ & $<.005$ & $<.17$ & $<.13$ & $<.026$ & $<.017$ & $<.002$ & $<.004$ \\
\hline YB-43-44-102 & 312003100370101 & $01-14-00$ & $<.11$ & $<.004$ & $<.002$ & $<.005$ & $<.17$ & $<.25$ & $<.026$ & $<.47$ & $<.002$ & $<.004$ \\
\hline YD-57-40-703 & 302306098050701 & 04-19-00 & $<.11$ & $<.004$ & $<.002$ & $<.005$ & $<.17$ & $<.13$ & $<.026$ & $<.017$ & $<.002$ & $<.004$ \\
\hline YD-58-50-830 & 300938097490601 & $11-03-99$ & $<.11$ & $<.004$ & $<.002$ & $<.005$ & $<.17$ & $<.13$ & $<.026$ & $<.017$ & $<.002$ & $<.004$ \\
\hline
\end{tabular}


Table 5. Concentrations of soluble pesticides in public water-supply wells sampled in Texas, November 1999-January 2001-Continued

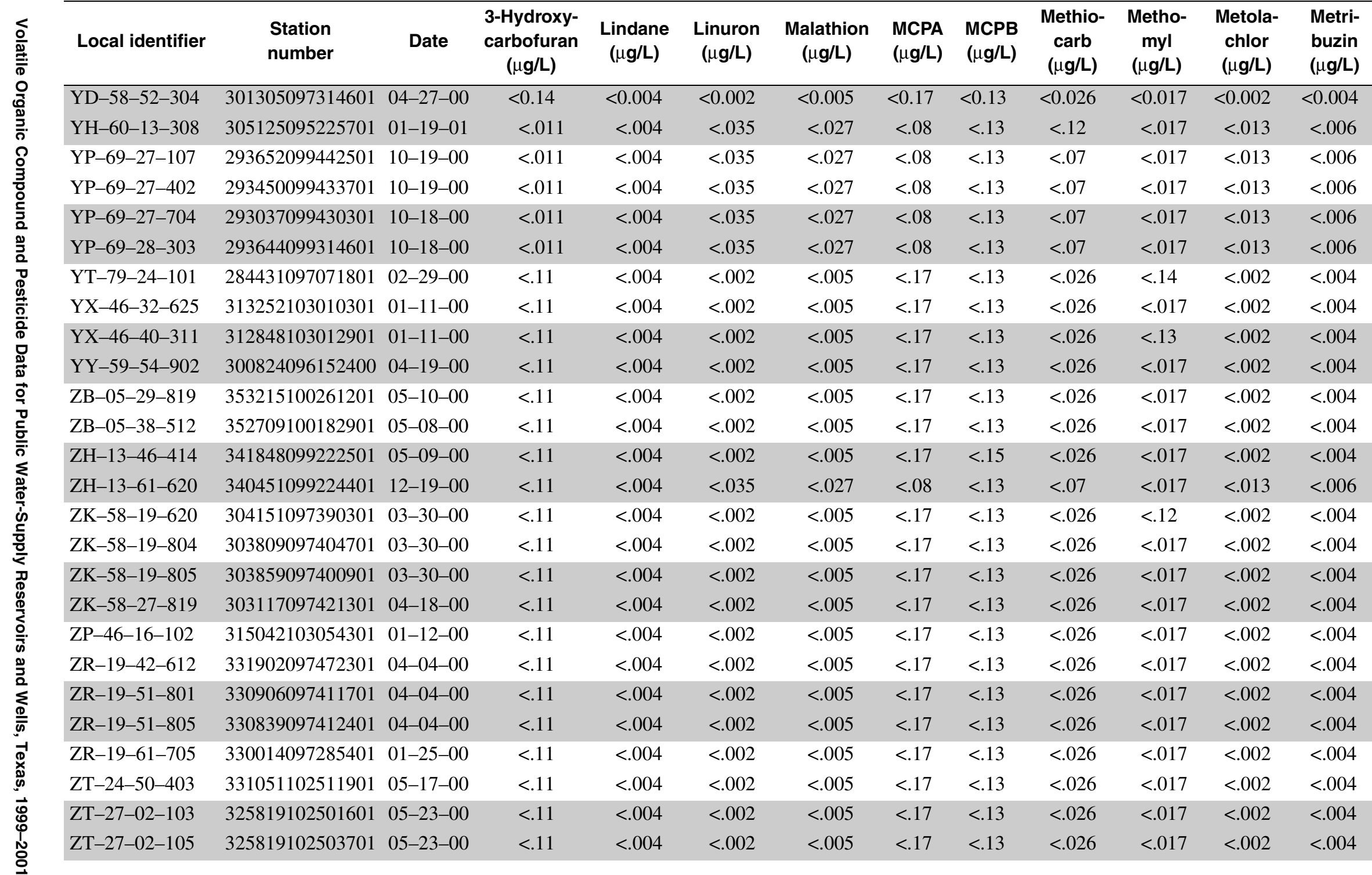


Table 5. Concentrations of soluble pesticides in public water-supply wells sampled in Texas, November 1999-January 2001—Continued

\begin{tabular}{|c|c|c|c|c|c|c|c|c|c|c|c|}
\hline Local identifier & $\begin{array}{l}\text { Station } \\
\text { number }\end{array}$ & Date & $\begin{array}{l}\text { Moli- } \\
\text { nate } \\
(\mu \mathrm{g} / \mathrm{L})\end{array}$ & $\begin{array}{c}\text { Napro- } \\
\text { pamide } \\
(\mu \mathrm{g} / \mathrm{L})\end{array}$ & $\begin{array}{c}\text { Neburon } \\
(\mu \mathrm{g} / \mathrm{L})\end{array}$ & $\begin{array}{c}\text { Norflur- } \\
\text { azon } \\
(\mu \mathrm{g} / \mathrm{L})\end{array}$ & $\begin{array}{c}\text { Oryzalin } \\
(\mu \mathrm{g} / L)\end{array}$ & $\begin{array}{c}\text { Oxamyl } \\
(\mu \mathrm{g} / \mathrm{L})\end{array}$ & $\begin{array}{l}\text { Para- } \\
\text { thion } \\
(\mu \mathrm{g} / \mathrm{L})\end{array}$ & $\begin{array}{c}\text { Parathion- } \\
\text { methyl } \\
(\mu \mathrm{g} / \mathrm{L})\end{array}$ & $\begin{array}{c}\text { Pebulate } \\
(\mu \mathrm{g} / \mathrm{L})\end{array}$ \\
\hline AB-27-37-103 & 322805102283801 & $05-17-00$ & $<0.004$ & $<0.003$ & $<0.07$ & $<0.042$ & $<0.31$ & $<0.018$ & $<0.004$ & $<0.006$ & $<0.004$ \\
\hline $\mathrm{AB}-27-37-105$ & 322826102280101 & $05-17-00$ & $<.004$ & $<.003$ & $<.07$ & $<.042$ & $<.31$ & $<.018$ & $<.004$ & $<.006$ & $<.004$ \\
\hline AK-06-52-506 & 351024101334401 & 05-04-00 & $<.004$ & $<.003$ & $<.15$ & $<.31$ & $<.32$ & $<4.55$ & $<.004$ & $<.006$ & $<.004$ \\
\hline AP-59-62-403 & 300259096195001 & 04-03-00 & $<.004$ & $<.003$ & $<.07$ & $<.042$ & $<.31$ & $<.018$ & $<.004$ & $<.006$ & $<.004$ \\
\hline AP-66-16-407 & 294903096061401 & $04-21-00$ & $<.004$ & $<.003$ & $<.07$ & $<.042$ & $<.31$ & $<.018$ & $<.004$ & $<.006$ & $<.004$ \\
\hline AR-10-51-408 & 341229102435401 & $05-01-00$ & $<.004$ & $<.003$ & $<.07$ & $<.042$ & $<.31$ & $<.018$ & $<.004$ & $<.006$ & $<.004$ \\
\hline AR-24-09-604 & 335000102545701 & 05-09-00 & $<.004$ & $<.003$ & $<.07$ & $<.042$ & $<.31$ & $<.018$ & $<.004$ & $<.006$ & $<.004$ \\
\hline AT-58-62-206 & 300646097193001 & $12-10-99$ & $<.004$ & $<.003$ & $<.07$ & $<.042$ & $<12.24$ & $<.018$ & $<.02$ & $<.006$ & $<.004$ \\
\hline AU-21-30-389 & 333612099163101 & $05-02-00$ & $<.004$ & $<.003$ & $<.07$ & $<.042$ & $<.31$ & $<.018$ & $<.004$ & $<.006$ & $<.004$ \\
\hline AX-58-04-604 & 305718097314401 & $03-22-00$ & $<.004$ & $<.003$ & $<.07$ & $<.042$ & $<.31$ & $<.018$ & $<.004$ & $<.006$ & $<.004$ \\
\hline $\mathrm{AX}-58-04-623$ & 305624097321101 & $03-22-00$ & $<.004$ & $<.003$ & $<.07$ & $<.042$ & $<.31$ & $<.018$ & $<.004$ & $<.006$ & $<.004$ \\
\hline AY $-68-35-105$ & 292842098425001 & $03-06-00$ & $<.004$ & $<.003$ & $<.07$ & $<.042$ & $<.31$ & $<.018$ & $<.004$ & $<.006$ & $<.004$ \\
\hline AZ-57-45-612 & 301923098225401 & $04-18-00$ & $<.004$ & $<.003$ & $<.07$ & $<.042$ & $<.31$ & $<.018$ & $<.004$ & $<.006$ & $<.004$ \\
\hline AZ-57-45-811 & 301633098252901 & 03-29-00 & $<.004$ & $<.003$ & $<.07$ & $<.042$ & $<.31$ & $<.018$ & $<.004$ & $<.006$ & $<.004$ \\
\hline BD-16-40-709 & 332301094061701 & 01-18-01 & $<.0016$ & $<.007$ & $<.017$ & $<.11$ & $<.32$ & $<.018$ & $<.007$ & $<.006$ & $<.0016$ \\
\hline BK-73-47-504 & 291911103124301 & $12-28-00$ & $<.0016$ & $<.007$ & $<.017$ & $<.042$ & $<.28$ & $<.018$ & $<.007$ & $<.006$ & $<.0016$ \\
\hline BK-73-52-905 & 290819103312601 & $12-28-00$ & $<.0016$ & $<.007$ & $<.017$ & $<.042$ & $<.28$ & $<.018$ & $<.007$ & $<.006$ & $<.0016$ \\
\hline BL-11-38-334 & 342841101155301 & $05-01-00$ & $<.004$ & $<.003$ & $<.07$ & $<.042$ & $<.31$ & $<.018$ & $<.004$ & $<.006$ & $<.004$ \\
\hline BL-11-48-507 & 341919101035201 & $05-02-00$ & $<.004$ & $<.003$ & $<.07$ & $<.042$ & $<.31$ & $<.018$ & $<.004$ & $<.006$ & $<.004$ \\
\hline BL-12-41-204 & 342109100570301 & $05-02-00$ & $<.004$ & $<.003$ & $<.07$ & $<.042$ & $<.31$ & $<.018$ & $<.004$ & $<.006$ & $<.004$ \\
\hline BR-41-01-244 & 315849098552101 & $04-05-00$ & $<.004$ & $<.003$ & $<.07$ & $<.042$ & $<.31$ & $<.018$ & $<.004$ & $<.006$ & $<.004$ \\
\hline BT-57-14-403 & 304939098202401 & 03-08-00 & $<.004$ & $<.003$ & $<.07$ & $<.042$ & $<.31$ & $<.018$ & $<.004$ & $<.006$ & $<.004$ \\
\hline BT-57-14-404 & 304927098211201 & 03-08-00 & $<.004$ & $<.003$ & $<.07$ & $<.042$ & $<.31$ & $<.018$ & $<.004$ & $<.006$ & $<.004$ \\
\hline BT-57-21-607 & 304125098224001 & $03-28-00$ & $<.004$ & $<.003$ & $<.07$ & $<.042$ & $<.31$ & $<.018$ & $<.004$ & $<.006$ & $<.004$ \\
\hline BT-57-21-608 & 304121098224201 & $03-28-00$ & $<.004$ & $<.003$ & $<.07$ & $<.042$ & $<.31$ & $<.018$ & $<.004$ & $<.006$ & $<.004$ \\
\hline BT $-57-23-110$ & 304406098130801 & 03-29-00 & $<.004$ & $<.003$ & $<.07$ & $<.042$ & $<.31$ & $<.018$ & $<.004$ & $<.006$ & $<.004$ \\
\hline BT-57-30-101 & 303548098211701 & $03-01-00$ & $<.004$ & $<.003$ & $<.07$ & $<.042$ & $<.31$ & $<.018$ & $<.004$ & $<.006$ & $<.004$ \\
\hline BU-67-10-910 & 294528097464401 & $03-21-00$ & $<.004$ & $<.003$ & $<.07$ & $<.042$ & $<.31$ & $<.018$ & $<.004$ & $<.006$ & $<.004$ \\
\hline
\end{tabular}


Table 5. Concentrations of soluble pesticides in public water-supply wells sampled in Texas, November 1999-January 2001-Continued

\begin{tabular}{|c|c|c|c|c|c|c|c|c|c|c|c|}
\hline Local identifier & $\begin{array}{l}\text { Station } \\
\text { number }\end{array}$ & Date & $\begin{array}{l}\text { Moli- } \\
\text { nate } \\
(\mu \mathrm{g} / \mathrm{L})\end{array}$ & $\begin{array}{c}\text { Napro- } \\
\text { pamide } \\
(\mu \mathrm{g} / \mathrm{L})\end{array}$ & $\begin{array}{c}\text { Neburon } \\
(\mu \mathrm{g} / \mathrm{L})\end{array}$ & $\begin{array}{c}\text { Norflur- } \\
\text { azon } \\
(\mu \mathrm{g} / \mathrm{L})\end{array}$ & $\begin{array}{c}\text { Oryzalin } \\
(\mu \mathrm{g} / \mathrm{L})\end{array}$ & $\begin{array}{c}\text { Oxamyl } \\
\text { ( } \mu \mathrm{g} / \mathrm{L})\end{array}$ & $\begin{array}{l}\text { Para- } \\
\text { thion } \\
(\mu \mathrm{g} / L)\end{array}$ & $\begin{array}{c}\text { Parathion- } \\
\text { methyl } \\
(\mu \mathrm{g} / \mathrm{L})\end{array}$ & $\begin{array}{c}\text { Pebulate } \\
\text { ( } \mu \mathrm{g} / \mathrm{L})\end{array}$ \\
\hline $\mathrm{BX}-30-55-936$ & 320939099093801 & $12-18-00$ & $<0.0016$ & $<0.007$ & $<0.017$ & $<0.15$ & $<0.28$ & $<0.018$ & $<0.007$ & $<0.006$ & $<0.0016$ \\
\hline DA-06-28-202 & 353704101343701 & $05-03-00$ & $<.004$ & $<.003$ & $<.07$ & $<.042$ & $<.31$ & $<.018$ & $<.004$ & $<.006$ & $<.004$ \\
\hline DL-14-62-604 & 340406098160701 & $05-10-00$ & $<.004$ & $<.003$ & $<.07$ & $<.042$ & $<.31$ & $<.018$ & $<.004$ & $<.006$ & $<.004$ \\
\hline DP-24-18-308 & 334352102450601 & 05-09-00 & $<.004$ & $<.003$ & $<.07$ & $<.042$ & $<.31$ & $<.018$ & $<.004$ & $<.006$ & $<.004$ \\
\hline DP-25-24-304 & 334245103011001 & $05-08-00$ & $<.004$ & $<.003$ & $<.07$ & $<.042$ & $<.31$ & $<.018$ & $<.004$ & $<.006$ & $<.004$ \\
\hline DU-12-06-609 & 345658100155501 & $05-08-00$ & $<.004$ & $<.003$ & $<.07$ & $<.042$ & $<.31$ & $<.018$ & $<.004$ & $<.006$ & $<.004$ \\
\hline DX-68-05-621 & 295520098242301 & $03-22-00$ & $<.004$ & $<.003$ & $<.07$ & $<.042$ & $<.31$ & $<.018$ & $<.004$ & $<.006$ & $<.004$ \\
\hline DX-68-06-405 & 295528098222301 & $03-22-00$ & $<.004$ & $<.003$ & $<.07$ & $<.042$ & $<.31$ & $<.018$ & $<.004$ & $<.006$ & $<.004$ \\
\hline DY-41-13-307 & 315105098241401 & $04-05-00$ & $<.004$ & $<.003$ & $<.07$ & $<.042$ & $<.31$ & $<.018$ & $<.004$ & $<.006$ & $<.004$ \\
\hline HH-45-35-505 & 312715102402201 & $01-12-00$ & $<.004$ & $<.003$ & $<.07$ & $<.042$ & $<12.84$ & $<.018$ & $<.004$ & $<.006$ & $<.004$ \\
\hline HP-12-62-802 & 340210100184901 & $05-05-00$ & $<.004$ & $<.003$ & -- & -- & -- & -- & $<.004$ & $<.006$ & $<.004$ \\
\hline HS-28-09-404 & 324810101591201 & $05-16-00$ & $<.004$ & $<.003$ & $<.07$ & $<.042$ & $<.31$ & $<.018$ & $<.004$ & $<.006$ & $<.004$ \\
\hline HT-07-63-204 & 350607102103401 & $05-03-00$ & $<.004$ & $<.003$ & $<.07$ & $<.042$ & $<.31$ & $<.018$ & $<.004$ & $<.006$ & $<.004$ \\
\hline HZ-77-34-606 & 282608099455301 & $03-28-00$ & $<.004$ & $<.003$ & $<.07$ & $<.042$ & $<.31$ & $<.018$ & $<.004$ & $<.006$ & $<.004$ \\
\hline JA-05-57-613 & 350254100535501 & $05-04-00$ & $<.004$ & $<.003$ & $<.07$ & $<.042$ & $<.31$ & $<.018$ & $<.004$ & $<.006$ & $<.004$ \\
\hline JA-12-12-404 & 344910100360401 & $05-02-00$ & $<.004$ & $<.003$ & $<.07$ & $<.042$ & $<.31$ & $<.018$ & $<.004$ & $<.006$ & $<.004$ \\
\hline JH-45-05-924 & 315245102240201 & $05-25-00$ & $<.004$ & $<.003$ & $<.07$ & $<.13$ & $<.31$ & $<.018$ & $<.004$ & $<.006$ & $<.004$ \\
\hline JL-49-04-116 & 315757106370201 & $01-06-00$ & $<.004$ & $<.003$ & $<.07$ & $<.042$ & $<13.37$ & $<.018$ & $<.004$ & $<.006$ & $<.004$ \\
\hline $\mathrm{JL}-49-04-423$ & 315708106362301 & $01-03-00$ & $<.004$ & $<.01$ & $<.07$ & $<.042$ & $<11.99$ & $<.018$ & $<.004$ & $<.006$ & $<.004$ \\
\hline JL-49-04-428 & 315517106361401 & $01-06-00$ & $<.004$ & $<.003$ & $<.07$ & $<.042$ & $<12.55$ & $<.018$ & $<.004$ & $<.006$ & $<.004$ \\
\hline JL-49-04-492 & 315623106360601 & $01-06-00$ & $<.004$ & $<.003$ & $<.07$ & $<.042$ & $<12.95$ & $<.018$ & $<.004$ & $<.006$ & $<.004$ \\
\hline JL-49-22-626 & 314145106163601 & $01-04-00$ & $<.004$ & $<.003$ & $<.07$ & $<.042$ & $<12.75$ & $<.018$ & $<.004$ & $<.006$ & $<.004$ \\
\hline JL-49-24-423 & 314107106063301 & $01-07-00$ & $<.004$ & $<.003$ & $<.07$ & $<.042$ & $<13.1$ & $<.018$ & $<.004$ & $<.006$ & $<.004$ \\
\hline JL-49-40-502 & 312648106044701 & $01-04-00$ & $<.004$ & $<.003$ & $<.07$ & $<.042$ & $<12.28$ & $<.018$ & $<.004$ & $<.006$ & $<.004$ \\
\hline JY-65-26-520 & 293314095474702 & 04-03-00 & $<.004$ & $<.003$ & $<.07$ & $<.042$ & $<.31$ & $<.018$ & $<.004$ & $<.006$ & $<.004$ \\
\hline KD-27-01-612 & 325523102542701 & $05-22-00$ & $<.004$ & $<.003$ & $<.07$ & $<.042$ & $<.31$ & $<.018$ & $<.004$ & $<.006$ & $<.004$ \\
\hline KK-57-42-709 & 301545098502801 & $07-06-00$ & $<.004$ & $<.003$ & $<.07$ & $<.042$ & $<.31$ & $<.018$ & $<.004$ & $<.006$ & $<.004$ \\
\hline $\mathrm{KK}-57-50-325$ & 301357098471901 & $03-28-00$ & $<.004$ & $<.003$ & $<.07$ & $<.042$ & $<.31$ & $<.018$ & $<.004$ & $<.006$ & $<.004$ \\
\hline KK-57-51-303 & 301411098383101 & $03-21-00$ & $<.004$ & $<.003$ & $<.07$ & $<.042$ & $<.31$ & $<.018$ & $<.004$ & $<.006$ & $<.004$ \\
\hline KK-57-52-107 & 301430098363401 & $03-21-00$ & $<.004$ & $<.003$ & $<.07$ & $<.042$ & $<.31$ & $<.45$ & $<.004$ & $<.006$ & $<.004$ \\
\hline
\end{tabular}


Table 5. Concentrations of soluble pesticides in public water-supply wells sampled in Texas, November 1999-January 2001-Continued

\begin{tabular}{|c|c|c|c|c|c|c|c|c|c|c|c|}
\hline Local identifier & $\begin{array}{l}\text { Station } \\
\text { number }\end{array}$ & Date & $\begin{array}{c}\text { Moli- } \\
\text { nate } \\
(\mu \mathrm{g} / \mathrm{L})\end{array}$ & $\begin{array}{c}\text { Napro- } \\
\text { pamide } \\
(\mu \mathrm{g} / \mathrm{L})\end{array}$ & $\begin{array}{l}\text { Neburon } \\
(\mu \mathrm{g} / \mathrm{L})\end{array}$ & $\begin{array}{l}\text { Norflur- } \\
\text { azon } \\
(\mu \mathrm{g} / \mathrm{L})\end{array}$ & $\begin{array}{c}\text { Oryzalin } \\
(\mu \mathrm{g} / \mathrm{L})\end{array}$ & $\begin{array}{c}\text { Oxamyl } \\
(\mu \mathrm{g} / \mathrm{L})\end{array}$ & $\begin{array}{l}\text { Para- } \\
\text { thion } \\
(\mu \mathrm{g} / \mathrm{L})\end{array}$ & $\begin{array}{c}\text { Parathion- } \\
\text { methyl } \\
(\mu \mathrm{g} / \mathrm{L})\end{array}$ & $\begin{array}{c}\text { Pebulate } \\
(\mu \mathrm{g} / \mathrm{L})\end{array}$ \\
\hline KP-79-23-101 & 284256097125001 & $02-29-00$ & $<0.004$ & $<0.003$ & $<0.07$ & $<0.042$ & $<0.31$ & $<0.018$ & $<0.004$ & $<0.006$ & $<0.004$ \\
\hline KT-18-11-405 & 334844096435701 & 04-19-00 & $<.004$ & $<.003$ & $<.07$ & $<.042$ & $<.31$ & $<.018$ & $<.004$ & $<.006$ & $<.004$ \\
\hline KT-18-11-406 & 334840096434001 & 04-19-00 & $<.004$ & $<.003$ & $<.07$ & $<.042$ & $<.31$ & $<.018$ & $<.004$ & $<.006$ & $<.004$ \\
\hline KT-18-28-703 & 333039096362901 & 04-19-00 & $<.004$ & $<.003$ & $<.07$ & $<.042$ & $<.31$ & $<.018$ & $<.004$ & $<.006$ & $<.004$ \\
\hline KY-11-57-602 & 340345101531801 & $05-03-00$ & $<.004$ & $<.003$ & $<.07$ & $<.042$ & $<.31$ & $<.018$ & $<.004$ & $<.006$ & $<.004$ \\
\hline KZ-12-19-104 & 344234100431601 & 05-09-00 & $<.004$ & $<.003$ & $<.15$ & $<.042$ & $<.31$ & $<.018$ & $<.004$ & $<.006$ & $<.004$ \\
\hline LD-13-44-907 & 341523099305701 & $12-19-00$ & $<.0016$ & $<.007$ & $<.017$ & $<.042$ & $<.28$ & $<.018$ & $<.007$ & $<.006$ & $<.0016$ \\
\hline LJ-60-64-807 & 300149095045001 & $05-04-00$ & $<.004$ & $<.003$ & $<.07$ & $<.042$ & $<.31$ & $<.018$ & $<.004$ & $<.006$ & $<.004$ \\
\hline LP-21-49-317 & 331233099545201 & $05-01-00$ & $<.004$ & $<.003$ & $<.07$ & $<.042$ & $<.31$ & $<.018$ & $<.004$ & $<.006$ & $<.004$ \\
\hline LX-24-40-405 & 332624102072901 & $05-17-00$ & $<.004$ & $<.003$ & $<.07$ & $<.042$ & $<.31$ & $<.018$ & $<.004$ & $<.006$ & $<.004$ \\
\hline LY-32-26-706 & 323204097500301 & $03-22-00$ & $<.004$ & $<.003$ & $<.07$ & $<.042$ & $<.31$ & $<.018$ & $<.004$ & $<.006$ & $<.004$ \\
\hline LY-32-34-208 & 322958097481301 & $03-21-00$ & $<.004$ & $<.003$ & $<.07$ & $<.042$ & $<.31$ & $<.018$ & $<.004$ & $<.006$ & $<.004$ \\
\hline LY-32-34-303 & 322928097461701 & 04-03-00 & $<.004$ & $<.003$ & $<.07$ & $<.042$ & $<.31$ & $<.018$ & $<.004$ & $<.006$ & $<.004$ \\
\hline LY-32-34-510 & 322537097485701 & $03-21-00$ & $<.004$ & $<.003$ & $<.07$ & $<.042$ & $<.31$ & $<.018$ & $<.004$ & $<.006$ & $<.004$ \\
\hline LY-32-34-609 & 322626097454801 & $03-21-00$ & $<.004$ & $<.003$ & $<.07$ & $<.042$ & $<.31$ & $<.018$ & $<.004$ & $<.006$ & $<.004$ \\
\hline PB-28-62-105 & 320649101215501 & $04-26-00$ & $<.004$ & $<.003$ & $<.07$ & $<.042$ & $<.31$ & $<.018$ & $<.004$ & $<.006$ & $<.004$ \\
\hline$P K-43-50-216$ & 311422100490201 & $12-20-00$ & $<.0016$ & $<.007$ & $<.017$ & $<.042$ & $<.329$ & $<.018$ & $<.007$ & $<.006$ & $<.0016$ \\
\hline PS-52-02-404 & 305502103504101 & $01-10-00$ & $<.004$ & $<.003$ & $<.07$ & $<.042$ & $<12.39$ & $<.018$ & $<.004$ & $<.006$ & $<.004$ \\
\hline PU-84-43-504 & 271841098402801 & 04-04-00 & $<.004$ & $<.003$ & $<.07$ & $<.042$ & $<.31$ & $<.018$ & $<.004$ & $<.006$ & $<.004$ \\
\hline PX-32-47-109 & 322201097141601 & $03-20-00$ & $<.004$ & $<.003$ & $<.07$ & $<.042$ & $<.31$ & $<.018$ & $<.004$ & $<.006$ & $<.004$ \\
\hline
\end{tabular}


Table 5. Concentrations of soluble pesticides in public water-supply wells sampled in Texas, November 1999-January 2001-Continued

\begin{tabular}{|c|c|c|c|c|c|c|c|c|c|c|c|}
\hline Local identifier & $\begin{array}{l}\text { Station } \\
\text { number }\end{array}$ & Date & $\begin{array}{c}\text { Moli- } \\
\text { nate } \\
(\mu \mathrm{g} / \mathrm{L})\end{array}$ & $\begin{array}{c}\text { Napro- } \\
\text { pamide } \\
(\mu \mathrm{g} / \mathrm{L})\end{array}$ & $\begin{array}{c}\text { Neburon } \\
(\mu \mathrm{g} / \mathrm{L})\end{array}$ & $\begin{array}{c}\text { Norflur- } \\
\text { azon } \\
(\mu \mathrm{g} / \mathrm{L})\end{array}$ & $\begin{array}{c}\text { Oryzalin } \\
(\mu g / L)\end{array}$ & $\begin{array}{c}\text { Oxamyl } \\
\text { ( } \mu \mathrm{g} / \mathrm{L})\end{array}$ & $\begin{array}{l}\text { Para- } \\
\text { thion } \\
(\mu \mathrm{g} / \mathrm{L})\end{array}$ & $\begin{array}{c}\text { Parathion- } \\
\text { methyl } \\
(\mu \mathrm{g} / \mathrm{L})\end{array}$ & $\begin{array}{c}\text { Pebulate } \\
\text { ( } \mu \mathrm{g} / \mathrm{L})\end{array}$ \\
\hline RU-10-60-606 & 340427102302801 & $05-02-00$ & $0<.004$ & $<0.003$ & $<0.07$ & $<0.042$ & $<0.31$ & $<0.018$ & $<0.004$ & $<0.006$ & $<0.004$ \\
\hline$R W-41-63-520$ & 310238098104001 & $03-23-00$ & $<.004$ & $<.003$ & $<.07$ & $<.042$ & $<.31$ & $<.018$ & $<.004$ & $<.006$ & $<.004$ \\
\hline SK-57-19-201 & 304323098421701 & $03-08-00$ & $<.004$ & $<.003$ & $<.07$ & $<.042$ & $<.31$ & $<.018$ & $<.004$ & $<.006$ & $<.004$ \\
\hline SK-57-34-201 & 303001098485201 & 03-09-00 & $<.004$ & $<.003$ & $<.07$ & $<.042$ & $<.31$ & $<.018$ & $<.004$ & $<.006$ & $<.004$ \\
\hline SP-23-17-417 & 334043101585701 & $05-10-00$ & $<.004$ & $<.003$ & $<.07$ & $<.042$ & $<.31$ & $<.018$ & $<.004$ & $<.006$ & $<.004$ \\
\hline SP-23-18-107 & 334306101504101 & $05-10-00$ & $<.004$ & $<.003$ & $<.07$ & $<.042$ & $<.31$ & $<.018$ & $<.004$ & $<.006$ & $<.004$ \\
\hline SP-23-19-812 & 333955101404601 & $05-10-00$ & $<.004$ & $<.003$ & $<.07$ & $<.042$ & $<.31$ & $<.018$ & $<.004$ & $<.006$ & $<.004$ \\
\hline SP-24-24-214 & 334419102045201 & $05-18-00$ & $<.004$ & $<.003$ & $<.07$ & $<.042$ & $<.31$ & $<.018$ & $<.004$ & $<.006$ & $<.004$ \\
\hline SR-23-41-602 & 331933101543701 & $05-15-00$ & $<.004$ & $<.003$ & $<.13$ & $<.042$ & $<.31$ & $<.018$ & $<.004$ & $<.006$ & $<.004$ \\
\hline SS-42-52-504 & 311202099321401 & $03-01-00$ & $<.004$ & $<.003$ & $<.07$ & $<.042$ & $<.31$ & $<.018$ & $<.004$ & $<.006$ & $<.004$ \\
\hline ST-40-39-304 & 312745097083301 & $03-23-00$ & $<.004$ & $<.003$ & $<.07$ & $<.042$ & $<.31$ & $<.018$ & $<.004$ & $<.006$ & $<.004$ \\
\hline SY-27-39-505 & 322555102105501 & $05-24-00$ & $<.004$ & $<.003$ & $<.07$ & $<.042$ & $<.31$ & $<.018$ & $<.004$ & $<.006$ & $<.004$ \\
\hline SY-28-42-803 & 321619101495001 & $05-23-00$ & $<.004$ & $<.003$ & $<.07$ & $<.042$ & $<.31$ & $<.018$ & $<.004$ & $<.006$ & $<.004$ \\
\hline SY-28-50-908 & 320816101474601 & $05-23-00$ & $<.004$ & $<.003$ & $<.07$ & $<.25$ & $<.31$ & $<.018$ & $<.004$ & $<.006$ & $<.004$ \\
\hline TB-76-03-605 & 285657100373801 & $03-28-00$ & $<.004$ & $<.003$ & $<.07$ & $<.042$ & $<.31$ & $<.018$ & $<.004$ & $<.006$ & $<.004$ \\
\hline TJ-27-63-712 & 320143102123101 & $12-20-00$ & $<.0016$ & $<.007$ & $<.017$ & $<.042$ & $<.28$ & $<.018$ & $<.007$ & $<.006$ & $<.0016$ \\
\hline TJ-28-57-702 & 320221101594901 & $05-25-00$ & $<.004$ & $<.003$ & $<.07$ & $<.042$ & $<.31$ & $<.018$ & $<.004$ & $<.006$ & $<.004$ \\
\hline TJ-28-57-903 & 320010101523701 & $05-23-00$ & $<.004$ & $<.003$ & $<.07$ & $<.042$ & $<.31$ & $<.018$ & $<.004$ & $<.006$ & $<.004$ \\
\hline TJ-45-07-407 & 315643102131101 & $05-24-00$ & $<.004$ & $<.003$ & $<.07$ & $<.042$ & $<.31$ & $<.018$ & $<.004$ & $<.006$ & $<.004$ \\
\hline TJ-45-07-606 & 315702102075401 & $05-25-00$ & $<.004$ & $<.003$ & $<.07$ & $<.042$ & $<.31$ & $<.018$ & $<.004$ & $<.006$ & $<.004$ \\
\hline TS-60-36-510 & 302701095331201 & 04-20-00 & $<.004$ & $<.003$ & $<.07$ & $<.042$ & $<.31$ & $<.018$ & $<.004$ & $<.006$ & $<.004$ \\
\hline TW-22-02-714 & 335416100502101 & $05-05-00$ & $<.004$ & $<.003$ & $<.07$ & $<.042$ & $<.31$ & $<.018$ & $<.004$ & $<.006$ & $<.004$ \\
\hline TW-22-02-716 & 335449100513101 & $12-19-00$ & $<.0016$ & $<.007$ & $<.017$ & $<.042$ & $<.28$ & $<.018$ & $<.007$ & $<.006$ & $<.0016$ \\
\hline UJ-62-49-712 & 300906093585201 & $04-28-00$ & $<.004$ & $<.003$ & $<.07$ & $<.042$ & $<.31$ & $<.018$ & $<.004$ & $<.006$ & $<.004$ \\
\hline UK-31-05-501 & 325545098255501 & $03-22-00$ & $<.004$ & $<.003$ & $<.07$ & $<.042$ & $<.31$ & $<.018$ & $<.004$ & $<.006$ & $<.004$ \\
\hline UK-31-24-501 & 324210098034701 & $03-22-00$ & $<.004$ & $<.003$ & $<.07$ & $<.042$ & $<.31$ & $<.018$ & $<.004$ & $<.006$ & $<.004$ \\
\hline UL-35-48-202 & 322207094034501 & $04-25-00$ & $<.004$ & $<.003$ & $<.07$ & $<.042$ & $<.31$ & $<.018$ & $<.004$ & $<.01$ & $<.004$ \\
\hline UP-32-10-604 & 324926097455001 & 02-09-00 & $<.004$ & $<.003$ & $<.07$ & $<.042$ & $<12.86$ & $<.018$ & $<.004$ & $<.006$ & $<.004$ \\
\hline UP-32-11-103 & 325005097440101 & $02-09-00$ & $<.004$ & $<.003$ & $<.07$ & $<.042$ & $<14.74$ & $<.018$ & $<.004$ & $<.006$ & $<.004$ \\
\hline UP-32-11-709 & 324649097442001 & 02-09-00 & $<.004$ & $<.003$ & $<.07$ & $<.042$ & $<13.44$ & $<.018$ & $<.004$ & $<.006$ & $<.004$ \\
\hline
\end{tabular}


Table 5. Concentrations of soluble pesticides in public water-supply wells sampled in Texas, November 1999-January 2001-Continued

\begin{tabular}{|c|c|c|c|c|c|c|c|c|c|c|c|}
\hline Local identifier & $\begin{array}{l}\text { Station } \\
\text { number }\end{array}$ & Date & $\begin{array}{c}\text { Moli- } \\
\text { nate } \\
(\mu \mathrm{g} / \mathrm{L})\end{array}$ & $\begin{array}{c}\text { Napro- } \\
\text { pamide } \\
(\mu \mathrm{g} / \mathrm{L})\end{array}$ & $\begin{array}{c}\text { Neburon } \\
(\mu \mathrm{g} / \mathrm{L})\end{array}$ & $\begin{array}{l}\text { Norflur- } \\
\text { azon } \\
(\mu \mathrm{g} / \mathrm{L})\end{array}$ & $\begin{array}{c}\text { Oryzalin } \\
(\mu \mathrm{g} / L)\end{array}$ & $\begin{array}{c}\text { Oxamyl } \\
(\mu g / L)\end{array}$ & $\begin{array}{l}\text { Para- } \\
\text { thion } \\
(\mu \mathrm{g} / \mathrm{L})\end{array}$ & $\begin{array}{c}\text { Parathion- } \\
\text { methyl } \\
(\mu \mathrm{g} / \mathrm{L})\end{array}$ & $\begin{array}{c}\text { Pebulate } \\
(\mu \mathrm{g} / \mathrm{L})\end{array}$ \\
\hline UP-32-11-801 & 324548097411701 & $02-10-00$ & $<0.004$ & $<0.003$ & $<0.07$ & $<0.042$ & $<13.76$ & $<0.018$ & $<0.004$ & $<0.006$ & $<0.004$ \\
\hline UP-32-28-403 & 323356097361801 & $04-10-00$ & $<.004$ & $<.003$ & $<.07$ & $<.042$ & $<.31$ & $<.018$ & $<.004$ & $<.02$ & $<.004$ \\
\hline US-53-08-601 & 305723102015801 & $03-14-00$ & $<.004$ & $<.003$ & $<.07$ & $<.042$ & $<.31$ & $<.018$ & $<.004$ & $<.006$ & $<.004$ \\
\hline US-54-18-503 & 304131101492401 & $03-14-00$ & $<.004$ & $<.003$ & $<.07$ & $<.042$ & $<.31$ & $<.018$ & $<.004$ & $<.006$ & $<.004$ \\
\hline UZ-44-37-505 & 312656101265101 & $01-13-00$ & $<.004$ & $<.003$ & $<.07$ & $<.042$ & $<12.47$ & $<.018$ & $<.004$ & $<.006$ & $<.004$ \\
\hline WB-16-18-704 & 333942094503001 & $04-26-00$ & $<.004$ & $<.003$ & $<.07$ & $<.042$ & $<.31$ & $<.018$ & $<.004$ & $<.006$ & $<.004$ \\
\hline WD-46-46-210 & 312041103174201 & $01-11-00$ & $<.004$ & $<.003$ & $<.07$ & $<.042$ & $<14.13$ & $<.18$ & $<.004$ & $<.006$ & $<.004$ \\
\hline WK-39-51-801 & 310956096402901 & 04-20-00 & $<.004$ & $<.003$ & $<.07$ & $<.042$ & $<.31$ & $<.018$ & $<.004$ & $<.006$ & $<.004$ \\
\hline WP-43-31-211 & 313551100110801 & $04-25-00$ & $<.004$ & $<.003$ & $<.07$ & $<.042$ & $<.31$ & $<.018$ & $<.004$ & $<.006$ & $<.004$ \\
\hline XT-11-26-923 & 343046101463701 & $05-01-00$ & $<.004$ & $<.003$ & $<.07$ & $<.042$ & $<.31$ & $<.018$ & $<.004$ & $<.006$ & $<.004$ \\
\hline XU-32-04-604 & 325525097304801 & $01-05-00$ & $<.004$ & $<.003$ & $<.07$ & $<.042$ & $<12.6$ & $<.018$ & $<.004$ & $<.006$ & $<.004$ \\
\hline XU-32-04-605 & 325525097304802 & $01-05-00$ & $<.004$ & $<.003$ & $<.07$ & $<.042$ & $<12.72$ & $<.018$ & $<.004$ & $<.006$ & $<.004$ \\
\hline XU-32-05-805 & 325335097272401 & 04-03-00 & $<.004$ & $<.003$ & $<.07$ & $<.042$ & $<.31$ & $<.018$ & $<.004$ & $<.006$ & $<.004$ \\
\hline XU-32-12-307 & 325101097313101 & $03-23-00$ & $<.004$ & $<.003$ & $<.07$ & $<.042$ & $<.31$ & $<.018$ & $<.004$ & $<.006$ & $<.004$ \\
\hline XU-32-13-405 & 324901097284701 & $01-24-00$ & $<.004$ & $<.003$ & $<.07$ & $<.042$ & $<14.85$ & $<.018$ & $<.004$ & $<.006$ & $<.004$ \\
\hline XU-32-13-503 & 324816097272401 & $01-24-00$ & $<.004$ & $<.003$ & $<.07$ & $<.042$ & $<13.63$ & $<.018$ & $<.004$ & $<.006$ & $<.004$ \\
\hline XU-32-23-701 & 323911097131001 & $03-20-00$ & $<.004$ & $<.003$ & $<.07$ & $<.042$ & $<.31$ & $<.018$ & $<.004$ & $<.006$ & $<.004$ \\
\hline$X Y-24-54-606$ & 331112102160201 & $05-17-00$ & $<.004$ & $<.003$ & $<.07$ & $<.042$ & $<.31$ & $<.018$ & $<.004$ & $<.006$ & $<.004$ \\
\hline YB-43-19-701 & 313943100425301 & $01-14-00$ & $<.004$ & $<.003$ & $<.07$ & $<.042$ & $<13.48$ & $<.018$ & $<.004$ & $<.006$ & $<.004$ \\
\hline YB-43-27-201 & 313638100400201 & $01-14-00$ & $<.004$ & $<.003$ & $<.07$ & $<.042$ & $<12.5$ & $<.018$ & $<.004$ & $<.006$ & $<.004$ \\
\hline
\end{tabular}


Table 5. Concentrations of soluble pesticides in public water-supply wells sampled in Texas, November 1999-January 2001-Continued

\begin{tabular}{|c|c|c|c|c|c|c|c|c|c|c|c|}
\hline Local identifier & $\begin{array}{l}\text { Station } \\
\text { number }\end{array}$ & Date & $\begin{array}{l}\text { Moli- } \\
\text { nate } \\
(\mu \mathrm{g} / \mathrm{L})\end{array}$ & $\begin{array}{c}\text { Napro- } \\
\text { pamide } \\
(\mu \mathrm{g} / \mathrm{L})\end{array}$ & $\begin{array}{c}\text { Neburon } \\
(\mu g / L)\end{array}$ & $\begin{array}{l}\text { Norflur- } \\
\text { azon } \\
(\mu \mathrm{g} / \mathrm{L})\end{array}$ & $\begin{array}{c}\text { Oryzalin } \\
(\mu g / L)\end{array}$ & $\begin{array}{c}\text { Oxamyl } \\
\text { ( } \mu \mathrm{g} / \mathrm{L})\end{array}$ & $\begin{array}{l}\text { Para- } \\
\text { thion } \\
(\mu \mathrm{g} / \mathrm{L})\end{array}$ & $\begin{array}{c}\text { Parathion- } \\
\text { methyl } \\
(\mu \mathrm{g} / \mathrm{L})\end{array}$ & $\begin{array}{c}\text { Pebulate } \\
\text { ( } \mu \mathrm{g} / \mathrm{L})\end{array}$ \\
\hline YD-58-52-304 & 301305097314601 & $04-27-00$ & $<0.004$ & $<0.003$ & $<0.07$ & $<0.042$ & $<0.31$ & $<0.018$ & $<0.004$ & $<0.006$ & $<0.004$ \\
\hline YH-60-13-308 & 305125095225701 & $01-19-01$ & $<.0016$ & $<.007$ & $<.017$ & $<.042$ & $<.28$ & $<.018$ & $<.007$ & $<.006$ & $<.0016$ \\
\hline YP-69-27-107 & 293652099442501 & $10-19-00$ & $<.0016$ & $<.007$ & $<.017$ & $<.042$ & $<.28$ & $<.018$ & $<.007$ & $<.006$ & $<.0016$ \\
\hline YP-69-27-402 & 293450099433701 & $10-19-00$ & $<.0016$ & $<.007$ & $<.017$ & $<.042$ & $<.28$ & $<.018$ & $<.007$ & $<.006$ & $<.0016$ \\
\hline YP-69-27-704 & 293037099430301 & $10-18-00$ & $<.0016$ & $<.007$ & $<.017$ & $<.042$ & $<.28$ & $<.018$ & $<.007$ & $<.006$ & $<.0016$ \\
\hline YP-69-28-303 & 293644099314601 & $10-18-00$ & $<.0016$ & $<.007$ & $<.017$ & $<.042$ & $<.28$ & $<.018$ & $<.007$ & $<.006$ & $<.0016$ \\
\hline YT-79-24-101 & 284431097071801 & $02-29-00$ & $<.004$ & $<.003$ & $<.07$ & $<.042$ & $<.31$ & $<.018$ & $<.004$ & $<.006$ & $<.004$ \\
\hline$Y X-46-32-625$ & 313252103010301 & $01-11-00$ & $<.004$ & $<.04$ & $<.07$ & $<.042$ & $<13.42$ & $<.018$ & $<.004$ & $<.006$ & $<.004$ \\
\hline$Y X-46-40-311$ & 312848103012901 & $01-11-00$ & $<.004$ & $<.003$ & $<.07$ & $<.042$ & $<19.48$ & $<.018$ & $<.004$ & $<.006$ & $<.004$ \\
\hline YY-59-54-902 & 300824096152400 & 04-19-00 & $<.004$ & $<.003$ & $<.07$ & $<.042$ & $<.31$ & $<.018$ & $<.004$ & $<.006$ & $<.004$ \\
\hline ZB-05-29-819 & 353215100261201 & $05-10-00$ & $<.004$ & $<.003$ & $<.07$ & $<.042$ & $<.31$ & $<.018$ & $<.004$ & $<.006$ & $<.004$ \\
\hline $\mathrm{ZB}-05-38-512$ & 352709100182901 & $05-08-00$ & $<.004$ & $<.003$ & $<.07$ & $<.042$ & $<.31$ & $<.018$ & $<.004$ & $<.006$ & $<.004$ \\
\hline $\mathrm{ZH}-13-46-414$ & 341848099222501 & $05-09-00$ & $<.004$ & $<.003$ & $<.07$ & $<.042$ & $<.31$ & $<.018$ & $<.004$ & $<.006$ & $<.004$ \\
\hline $\mathrm{ZH}-13-61-620$ & 340451099224401 & $12-19-00$ & $<.0016$ & $<.007$ & $<.017$ & $<.042$ & $<.336$ & $<.018$ & $<.007$ & $<.006$ & $<.0016$ \\
\hline ZK-58-19-620 & 304151097390301 & $03-30-00$ & $<.004$ & $<.003$ & $<.07$ & $<.042$ & $<.31$ & $<.018$ & $<.004$ & $<.006$ & $<.004$ \\
\hline ZK-58-19-804 & 303809097404701 & $03-30-00$ & $<.004$ & $<.003$ & $<.07$ & $<.042$ & $<.31$ & $<.018$ & $<.004$ & $<.006$ & $<.004$ \\
\hline ZK-58-19-805 & 303859097400901 & $03-30-00$ & $<.004$ & $<.003$ & $<.07$ & $<.042$ & $<.31$ & $<.018$ & $<.004$ & $<.006$ & $<.004$ \\
\hline ZK-58-27-819 & 303117097421301 & $04-18-00$ & $<.004$ & $<.003$ & $<.07$ & $<.042$ & $<.31$ & $<.018$ & $<.004$ & $<.006$ & $<.004$ \\
\hline ZP-46-16-102 & 315042103054301 & $01-12-00$ & $<.004$ & $<.003$ & $<.07$ & $<.042$ & $<12.16$ & $<.018$ & $<.004$ & $<.006$ & $<.004$ \\
\hline ZR-19-42-612 & 331902097472301 & 04-04-00 & $<.004$ & $<.003$ & $<.07$ & $<.042$ & $<.31$ & $<.018$ & $<.004$ & $<.006$ & $<.004$ \\
\hline ZR-19-51-801 & 330906097411701 & $04-04-00$ & $<.004$ & $<.003$ & $<.07$ & $<.042$ & $<.31$ & $<.018$ & $<.004$ & $<.006$ & $<.004$ \\
\hline ZR-19-51-805 & 330839097412401 & $04-04-00$ & $<.004$ & $<.003$ & $<.07$ & $<.042$ & $<.31$ & $<.018$ & $<.004$ & $<.006$ & $<.004$ \\
\hline ZR-19-61-705 & 330014097285401 & $01-25-00$ & $<.004$ & $<.003$ & $<.07$ & $<.042$ & $<15.76$ & $<.018$ & $<.004$ & $<.006$ & $<.004$ \\
\hline $\mathrm{ZT}-24-50-403$ & 331051102511901 & $05-17-00$ & $<.004$ & $<.003$ & $<.07$ & $<.042$ & $<.31$ & $<.018$ & $<.004$ & $<.006$ & $<.004$ \\
\hline ZT-27-02-103 & 325819102501601 & $05-23-00$ & $<.004$ & $<.003$ & $<.07$ & $<.042$ & $<.31$ & $<.018$ & $<.004$ & $<.006$ & $<.004$ \\
\hline $\mathrm{ZT}-27-02-105$ & 325819102503701 & $05-23-00$ & $<.004$ & $<.003$ & $<.07$ & $<.042$ & $<.31$ & $<.018$ & $<.004$ & $<.006$ & $<.004$ \\
\hline
\end{tabular}


Table 5. Concentrations of soluble pesticides in public water-supply wells sampled in Texas, November 1999-January 2001-Continued

\begin{tabular}{|c|c|c|c|c|c|c|c|c|c|c|}
\hline Local identifier & $\begin{array}{l}\text { Station } \\
\text { number }\end{array}$ & Date & $\begin{array}{l}\text { Pendi- } \\
\text { methalin } \\
(\mu \mathrm{g} / \mathrm{L})\end{array}$ & $\begin{array}{l}\text { Perme- } \\
\text { thrin } \\
(\mu \mathrm{g} / \mathrm{L})\end{array}$ & $\begin{array}{c}\text { Phorate } \\
(\mu \mathrm{g} / \mathrm{L})\end{array}$ & $\begin{array}{l}\text { Picloram } \\
(\mu \mathrm{g} / \mathrm{L})\end{array}$ & $\begin{array}{c}p, p^{\prime}- \\
\text { DDE } \\
(\mu \mathrm{g} / L)\end{array}$ & $\begin{array}{l}\text { Pro- } \\
\text { meton } \\
(\mu \mathrm{g} / \mathrm{L})\end{array}$ & $\begin{array}{l}\text { Pronamide } \\
(\mu \mathrm{g} / \mathrm{L})\end{array}$ & $\begin{array}{l}\text { Propa- } \\
\text { chlor } \\
(\mu \mathrm{g} / \mathrm{L})\end{array}$ \\
\hline $\mathrm{AB}-27-37-103$ & 322805102283801 & $05-17-00$ & $<0.004$ & $<0.005$ & $<0.002$ & $<0.05$ & $<0.006$ & $<0.018$ & $<0.003$ & $<0.007$ \\
\hline $\mathrm{AB}-27-37-105$ & 322826102280101 & $05-17-00$ & $<.004$ & $<.005$ & $<.002$ & $<.05$ & $<.006$ & $<.018$ & $<.003$ & $<.007$ \\
\hline AK-06-52-506 & 351024101334401 & $05-04-00$ & $<.004$ & $<.005$ & $<.002$ & $<.05$ & $<.006$ & $<.018$ & $<.003$ & $<.007$ \\
\hline AP-59-62-403 & 300259096195001 & 04-03-00 & $<.004$ & $<.005$ & $<.002$ & $<.05$ & $<.006$ & $<.018$ & $<.003$ & $<.007$ \\
\hline AP-66-16-407 & 294903096061401 & $04-21-00$ & $<.004$ & $<.005$ & $<.002$ & $<.05$ & $<.006$ & $<.018$ & $<.003$ & $<.007$ \\
\hline AR-10-51-408 & 341229102435401 & $05-01-00$ & $<.004$ & $<.005$ & $<.002$ & $<.05$ & $<.006$ & $<.018$ & $<.003$ & $<.007$ \\
\hline AR-24-09-604 & 335000102545701 & 05-09-00 & $<.004$ & $<.005$ & $<.002$ & $<.05$ & $<.006$ & $<.018$ & $<.003$ & $<.007$ \\
\hline AT-58-62-206 & 300646097193001 & $12-10-99$ & $<.004$ & $<.005$ & $<.002$ & $<.05$ & $<.01$ & $<.018$ & $<.003$ & $<.007$ \\
\hline AU-21-30-389 & 333612099163101 & $05-02-00$ & $<.004$ & $<.005$ & $<.002$ & $<.05$ & $<.006$ & .020 & $<.003$ & $<.007$ \\
\hline$A X-58-04-604$ & 305718097314401 & $03-22-00$ & $<.004$ & $<.005$ & $<.002$ & $<.05$ & $<.006$ & E.017 & $<.003$ & $<.007$ \\
\hline $\mathrm{AX}-58-04-623$ & 305624097321101 & $03-22-00$ & $<.004$ & $<.005$ & $<.002$ & $<.05$ & $<.006$ & $<.018$ & $<.003$ & $<.007$ \\
\hline AY $-68-35-105$ & 292842098425001 & 03-06-00 & $<.004$ & $<.005$ & $<.002$ & $<.05$ & $<.006$ & $<.018$ & $<.003$ & $<.007$ \\
\hline AZ-57-45-612 & 301923098225401 & $04-18-00$ & $<.004$ & $<.005$ & $<.002$ & $<.05$ & $<.006$ & $<.018$ & $<.003$ & $<.007$ \\
\hline AZ-57-45-811 & 301633098252901 & 03-29-00 & $<.004$ & $<.005$ & $<.002$ & $<.05$ & $<.006$ & $<.018$ & $<.003$ & $<.007$ \\
\hline BD-16-40-709 & 332301094061701 & 01-18-01 & $<.01$ & $<.006$ & $<.011$ & $<.09$ & $<.0025$ & $<.015$ & $<.0041$ & $<.01$ \\
\hline BK-73-47-504 & 291911103124301 & $12-28-00$ & $<.01$ & $<.006$ & $<.011$ & $<.09$ & $<.0025$ & $<.015$ & $<.0041$ & $<.01$ \\
\hline BK-73-52-905 & 290819103312601 & $12-28-00$ & $<.01$ & $<.006$ & $<.011$ & $<.09$ & $<.0025$ & $<.015$ & $<.0041$ & $<.01$ \\
\hline BL-11-38-334 & 342841101155301 & $05-01-00$ & $<.004$ & $<.005$ & $<.002$ & $<.05$ & $<.006$ & $<.018$ & $<.003$ & $<.007$ \\
\hline BL-11-48-507 & 341919101035201 & $05-02-00$ & $<.004$ & $<.005$ & $<.002$ & $<.05$ & $<.006$ & $<.018$ & $<.003$ & $<.007$ \\
\hline BL-12-41-204 & 342109100570301 & $05-02-00$ & $<.004$ & $<.005$ & $<.002$ & $<.05$ & $<.006$ & $<.018$ & $<.003$ & $<.007$ \\
\hline BR-41-01-244 & 315849098552101 & $04-05-00$ & $<.004$ & $<.005$ & $<.002$ & $<.05$ & $<.006$ & E.006 & $<.003$ & $<.007$ \\
\hline BT-57-14-403 & 304939098202401 & $03-08-00$ & $<.004$ & $<.005$ & $<.002$ & $<.05$ & $<.006$ & $<.018$ & $<.003$ & $<.007$ \\
\hline BT-57-14-404 & 304927098211201 & $03-08-00$ & $<.004$ & $<.005$ & $<.002$ & $<.05$ & $<.006$ & $<.018$ & $<.003$ & $<.007$ \\
\hline BT-57-21-607 & 304125098224001 & $03-28-00$ & $<.004$ & $<.005$ & $<.002$ & $<.05$ & $<.006$ & $<.018$ & $<.003$ & $<.007$ \\
\hline BT-57-21-608 & 304121098224201 & $03-28-00$ & $<.004$ & $<.005$ & $<.002$ & $<.05$ & $<.006$ & $<.018$ & $<.003$ & $<.007$ \\
\hline BT-57-23-110 & 304406098130801 & 03-29-00 & $<.004$ & $<.005$ & $<.002$ & .106 & $<.006$ & .036 & $<.003$ & $<.007$ \\
\hline BT-57-30-101 & 303548098211701 & 03-01-00 & $<.004$ & $<.005$ & $<.002$ & $<.05$ & $<.006$ & $<.018$ & $<.003$ & $<.007$ \\
\hline BU-67-10-910 & 294528097464401 & $03-21-00$ & $<.004$ & $<.005$ & $<.002$ & $<.05$ & $<.006$ & $<.018$ & $<.003$ & $<.007$ \\
\hline
\end{tabular}


Table 5. Concentrations of soluble pesticides in public water-supply wells sampled in Texas, November 1999-January 2001-Continued

\begin{tabular}{|c|c|c|c|c|c|c|c|c|c|c|}
\hline Local identifier & $\begin{array}{l}\text { Station } \\
\text { number }\end{array}$ & Date & $\begin{array}{c}\text { Pendi- } \\
\text { methalin } \\
(\mu \mathrm{g} / \mathrm{L})\end{array}$ & $\begin{array}{c}\text { Perme- } \\
\text { thrin } \\
(\mu \mathrm{g} / \mathrm{L})\end{array}$ & $\begin{array}{l}\text { Phorate } \\
(\mu \mathrm{g} / \mathrm{L})\end{array}$ & $\begin{array}{c}\text { Picloram } \\
(\mu \mathrm{g} / \mathrm{L})\end{array}$ & $\begin{array}{c}p, p^{\prime}- \\
\text { DDE } \\
(\mu g / L)\end{array}$ & $\begin{array}{c}\text { Pro- } \\
\text { meton } \\
(\mu g / L)\end{array}$ & $\begin{array}{l}\text { Pronamide } \\
(\mu \mathrm{g} / \mathrm{L})\end{array}$ & $\begin{array}{c}\text { Propa- } \\
\text { chlor } \\
(\mu \mathrm{g} / \mathrm{L})\end{array}$ \\
\hline BX-30-55-936 & 320939099093801 & $12-18-00$ & $<0.01$ & $<0.006$ & $<0.011$ & $<0.09$ & $<0.0025$ & $<0.015$ & $<0.0041$ & $<0.01$ \\
\hline DA-06-28-202 & 353704101343701 & $05-03-00$ & $<.004$ & $<.005$ & $<.002$ & $<.05$ & $<.006$ & $<.018$ & $<.003$ & $<.007$ \\
\hline DL-14-62-604 & 340406098160701 & $05-10-00$ & $<.004$ & $<.005$ & $<.002$ & $<.05$ & $<.006$ & $<.018$ & $<.003$ & $<.007$ \\
\hline DP-24-18-308 & 334352102450601 & 05-09-00 & $<.004$ & $<.005$ & $<.002$ & $<.05$ & $<.006$ & $<.018$ & $<.003$ & $<.007$ \\
\hline DP-25-24-304 & 334245103011001 & $05-08-00$ & $<.004$ & $<.005$ & $<.002$ & $<.05$ & $<.006$ & $<.018$ & $<.003$ & $<.007$ \\
\hline DU-12-06-609 & 345658100155501 & $05-08-00$ & $<.004$ & $<.005$ & $<.002$ & $<.05$ & $<.006$ & $<.018$ & $<.003$ & $<.007$ \\
\hline DX-68-05-621 & 295520098242301 & $03-22-00$ & $<.004$ & $<.005$ & $<.002$ & $<.05$ & $<.01$ & $<.018$ & $<.003$ & $<.007$ \\
\hline DX-68-06-405 & 295528098222301 & $03-22-00$ & $<.004$ & $<.005$ & $<.002$ & $<.05$ & $<.006$ & $<.018$ & $<.003$ & $<.007$ \\
\hline DY-41-13-307 & 315105098241401 & $04-05-00$ & $<.004$ & $<.005$ & $<.002$ & .581 & $<.006$ & $<.018$ & $<.003$ & $<.007$ \\
\hline HH-45-35-505 & 312715102402201 & $01-12-00$ & $<.004$ & $<.005$ & $<.002$ & $<.05$ & $<.006$ & $<.018$ & $<.003$ & $<.007$ \\
\hline HP-12-62-802 & 340210100184901 & $05-05-00$ & $<.004$ & $<.005$ & $<.002$ & $<.05$ & $<.006$ & E.009 & $<.003$ & $<.007$ \\
\hline HS-28-09-404 & 324810101591201 & $05-16-00$ & $<.004$ & $<.005$ & $<.002$ & $<.05$ & $<.006$ & $<.018$ & $<.003$ & $<.007$ \\
\hline HТ-07-63-204 & 350607102103401 & $05-03-00$ & $<.004$ & $<.005$ & $<.002$ & $<.05$ & $<.006$ & $<.018$ & $<.003$ & $<.007$ \\
\hline HZ-77-34-606 & 282608099455301 & $03-28-00$ & $<.004$ & $<.005$ & $<.002$ & $<.05$ & $<.006$ & $<.018$ & $<.003$ & $<.007$ \\
\hline JA-05-57-613 & 350254100535501 & 05-04-00 & $<.004$ & $<.005$ & $<.002$ & $<.05$ & $<.006$ & $<.018$ & $<.003$ & $<.007$ \\
\hline JA-12-12-404 & 344910100360401 & $05-02-00$ & $<.004$ & $<.005$ & $<.002$ & $<.05$ & $<.006$ & $<.018$ & $<.003$ & $<.007$ \\
\hline JH-45-05-924 & 315245102240201 & $05-25-00$ & $<.004$ & $<.005$ & $<.002$ & $<.05$ & $<.006$ & .016 & $<.003$ & $<.007$ \\
\hline JL-49-04-116 & 315757106370201 & $01-06-00$ & $<.004$ & $<.005$ & $<.002$ & $<.05$ & $<.006$ & $<.018$ & $<.003$ & $<.007$ \\
\hline JL-49-04-423 & 315708106362301 & $01-03-00$ & $<.004$ & $<.005$ & $<.002$ & $<.05$ & $<.006$ & $<.018$ & $<.003$ & $<.007$ \\
\hline JL-49-04-428 & 315517106361401 & $01-06-00$ & $<.004$ & $<.005$ & $<.002$ & $<.05$ & $<.006$ & $<.018$ & $<.003$ & $<.007$ \\
\hline JL-49-04-492 & 315623106360601 & $01-06-00$ & $<.004$ & $<.005$ & $<.002$ & $<.05$ & $<.006$ & $<.018$ & $<.003$ & $<.007$ \\
\hline JL-49-22-626 & 314145106163601 & $01-04-00$ & $<.004$ & $<.005$ & $<.002$ & $<.05$ & $<.006$ & $<.018$ & $<.003$ & $<.007$ \\
\hline JL-49-24-423 & 314107106063301 & $01-07-00$ & $<.004$ & $<.005$ & $<.002$ & $<.05$ & $<.006$ & $<.018$ & $<.003$ & $<.007$ \\
\hline JL-49-40-502 & 312648106044701 & $01-04-00$ & $<.004$ & $<.005$ & $<.002$ & $<.05$ & $<.006$ & $<.018$ & $<.003$ & $<.007$ \\
\hline JY-65-26-520 & 293314095474702 & 04-03-00 & $<.004$ & $<.005$ & $<.002$ & $<.05$ & $<.006$ & $<.018$ & $<.003$ & $<.007$ \\
\hline KD-27-01-612 & 325523102542701 & $05-22-00$ & $<.004$ & $<.005$ & $<.002$ & $<.05$ & $<.006$ & $<.018$ & $<.003$ & $<.007$ \\
\hline KK-57-42-709 & 301545098502801 & $07-06-00$ & $<.004$ & $<.005$ & $<.002$ & $<.05$ & $<.006$ & $<.018$ & $<.003$ & $<.007$ \\
\hline KK-57-50-325 & 301357098471901 & $03-28-00$ & $<.004$ & $<.005$ & $<.002$ & $<.05$ & $<.006$ & $<.018$ & $<.003$ & $<.007$ \\
\hline KK-57-51-303 & 301411098383101 & $03-21-00$ & $<.004$ & $<.005$ & $<.002$ & $<.05$ & $<.006$ & $<.018$ & $<.003$ & $<.007$ \\
\hline KK-57-52-107 & 301430098363401 & $03-21-00$ & $<.004$ & $<.005$ & $<.002$ & $<.05$ & $<.006$ & $<.018$ & $<.003$ & $<.007$ \\
\hline
\end{tabular}


Table 5. Concentrations of soluble pesticides in public water-supply wells sampled in Texas, November 1999-January 2001-Continued

\begin{tabular}{|c|c|c|c|c|c|c|c|c|c|c|}
\hline Local identifier & $\begin{array}{l}\text { Station } \\
\text { number }\end{array}$ & Date & $\begin{array}{l}\text { Pendi- } \\
\text { methalin } \\
(\mu \mathrm{g} / \mathrm{L})\end{array}$ & $\begin{array}{l}\text { Perme- } \\
\text { thrin } \\
(\mu \mathrm{g} / \mathrm{L})\end{array}$ & $\begin{array}{l}\text { Phorate } \\
(\mu \mathrm{g} / \mathrm{L})\end{array}$ & $\begin{array}{c}\text { Picloram } \\
(\mu g / L)\end{array}$ & $\begin{array}{c}p, p^{\prime}- \\
\text { DDE } \\
(\mu \mathrm{g} / \mathrm{L})\end{array}$ & $\begin{array}{l}\text { Pro- } \\
\text { meton } \\
(\mu \mathrm{g} / \mathrm{L})\end{array}$ & $\begin{array}{l}\text { Pronamide } \\
\qquad(\mu \mathrm{g} / L)\end{array}$ & $\begin{array}{l}\text { Propa- } \\
\text { chlor } \\
\text { ( } \mu \mathrm{g} / \mathrm{L})\end{array}$ \\
\hline KP-79-23-101 & 284256097125001 & 02-29-00 & $<0.004$ & $<0.005$ & $<0.002$ & $<.05$ & $<.006$ & $<.018$ & $<.003$ & $<.007$ \\
\hline KS-05-52-105 & 351428100352601 & 05-04-00 & $<.004$ & $<.005$ & $<.002$ & $<.05$ & $<.006$ & $<.018$ & $<.003$ & $<.007$ \\
\hline KT-18-11-405 & 334844096435701 & 04-19-00 & $<.004$ & $<.005$ & $<.002$ & $<.05$ & $<.006$ & $<.018$ & $<.003$ & $<.007$ \\
\hline KT-18-11-406 & 334840096434001 & 04-19-00 & $<.004$ & $<.005$ & $<.002$ & $<.05$ & $<.006$ & .044 & $<.003$ & $<.007$ \\
\hline KT-18-28-703 & 333039096362901 & 04-19-00 & $<.004$ & $<.005$ & $<.002$ & $<.05$ & $<.006$ & $<.018$ & $<.003$ & $<.007$ \\
\hline KY-11-49-512 & 341116101574601 & 05-04-00 & $<.004$ & $<.005$ & $<.002$ & $<.05$ & $<.006$ & $<.018$ & $<.003$ & $<.007$ \\
\hline $\mathrm{KY}-11-51-416$ & 341032101423801 & $05-04-00$ & $<.004$ & $<.005$ & $<.002$ & $<.05$ & $<.006$ & $<.018$ & $<.003$ & $<.007$ \\
\hline KY-11-57-602 & 340345101531801 & $05-03-00$ & $<.004$ & $<.005$ & $<.002$ & .115 & $<.006$ & $<.018$ & $<.003$ & $<.007$ \\
\hline KZ-12-19-104 & 344234100431601 & 05-09-00 & $<.004$ & $<.005$ & $<.002$ & $<.05$ & $<.006$ & $<.018$ & $<.003$ & $<.007$ \\
\hline LD-13-44-907 & 341523099305701 & $12-19-00$ & $<.01$ & $<.006$ & $<.011$ & $<.09$ & $<.0025$ & $<.015$ & $<.0041$ & $<.01$ \\
\hline LJ-60-64-807 & 300149095045001 & $05-04-00$ & $<.004$ & $<.005$ & $<.002$ & $<.05$ & .002 & $<.018$ & $<.003$ & $<.007$ \\
\hline LP-21-49-317 & 331233099545201 & $05-01-00$ & $<.004$ & $<.005$ & $<.002$ & $<.05$ & $<.006$ & $<.018$ & $<.003$ & $<.007$ \\
\hline LR-67-09-112 & 295137097585801 & $03-21-00$ & $<.004$ & $<.005$ & $<.002$ & $<.05$ & $<.006$ & $<.018$ & $<.003$ & $<.007$ \\
\hline LX-24-15-612 & 334857102092301 & $05-11-00$ & $<.004$ & $<.005$ & $<.002$ & $<.05$ & $<.006$ & $<.018$ & $<.003$ & $<.007$ \\
\hline LX-24-29-306 & 333536102223101 & $05-09-00$ & $<.004$ & $<.005$ & $<.002$ & $<.05$ & $<.006$ & $<.018$ & $<.003$ & $<.007$ \\
\hline LX-24-37-405 & 332714102285401 & $05-16-00$ & $<.004$ & $<.005$ & $<.002$ & $<.05$ & $<.006$ & $<.018$ & $<.003$ & $<.007$ \\
\hline LX-24-40-405 & 332624102072901 & $05-17-00$ & $<.004$ & $<.005$ & $<.002$ & $<.05$ & $<.006$ & $<.018$ & $<.003$ & $<.007$ \\
\hline LY-32-26-706 & 323204097500301 & $03-22-00$ & $<.004$ & $<.005$ & $<.002$ & $<.05$ & $<.006$ & $<.018$ & $<.003$ & $<.007$ \\
\hline LY-32-34-208 & 322958097481301 & $03-21-00$ & $<.004$ & $<.005$ & $<.002$ & $<.05$ & $<.006$ & $<.018$ & $<.003$ & $<.007$ \\
\hline LY-32-34-303 & 322928097461701 & 04-03-00 & $<.004$ & $<.005$ & $<.002$ & $<.05$ & $<.006$ & $<.018$ & $<.003$ & $<.007$ \\
\hline LY-32-34-510 & 322537097485701 & $03-21-00$ & $<.004$ & $<.005$ & $<.002$ & $<.05$ & $<.006$ & $<.018$ & $<.003$ & $<.007$ \\
\hline LY-32-34-609 & 322626097454801 & $03-21-00$ & $<.004$ & $<.005$ & $<.002$ & $<.05$ & $<.006$ & $<.018$ & $<.003$ & $<.007$ \\
\hline PB-28-62-105 & 320649101215501 & $04-26-00$ & $<.004$ & $<.005$ & $<.002$ & $<.05$ & $<.006$ & E.012 & $<.003$ & $<.007$ \\
\hline PK-43-50-216 & 311422100490201 & $12-20-00$ & $<.01$ & $<.006$ & $<.011$ & $<.09$ & $<.0025$ & $<.015$ & $<.0041$ & $<.01$ \\
\hline PS-52-02-404 & 305502103504101 & 01-10-00 & $<.004$ & $<.005$ & $<.002$ & $<.05$ & $<.006$ & $<.018$ & $<.003$ & $<.007$ \\
\hline PU-84-43-504 & 271841098402801 & 04-04-00 & $<.004$ & $<.005$ & $<.002$ & $<.05$ & $<.006$ & $<.018$ & $<.003$ & $<.007$ \\
\hline PX-32-47-109 & 322201097141601 & $03-20-00$ & $<.004$ & $<.005$ & $<.002$ & $<.05$ & $<.006$ & $<.018$ & $<.003$ & $<.007$ \\
\hline PZ-79-10-807 & 284713097493901 & $02-23-00$ & $<.004$ & $<.005$ & $<.002$ & $<.05$ & $<.006$ & $<.018$ & $<.003$ & $<.007$ \\
\hline RS-21-35-103 & 332854099441101 & 05-01-00 & $<.004$ & $<.005$ & $<.002$ & $<.05$ & $<.006$ & $<.018$ & $<.003$ & $<.007$ \\
\hline RU-10-53-312 & 341418102243401 & $05-02-00$ & $<.004$ & $<.005$ & $<.002$ & $<.05$ & $<.006$ & $<.018$ & $<.003$ & $<.007$ \\
\hline
\end{tabular}


Table 5. Concentrations of soluble pesticides in public water-supply wells sampled in Texas, November 1999-January 2001-Continued

\begin{tabular}{|c|c|c|c|c|c|c|c|c|c|c|}
\hline Local identifier & $\begin{array}{l}\text { Station } \\
\text { number }\end{array}$ & Date & $\begin{array}{c}\text { Pendi- } \\
\text { methalin } \\
(\mu \mathrm{g} / \mathrm{L})\end{array}$ & $\begin{array}{c}\text { Perme- } \\
\text { thrin } \\
(\mu \mathrm{g} / \mathrm{L})\end{array}$ & $\begin{array}{l}\text { Phorate } \\
(\mu \mathrm{g} / \mathrm{L})\end{array}$ & $\begin{array}{c}\text { Picloram } \\
(\mu \mathrm{g} / \mathrm{L})\end{array}$ & $\begin{array}{c}p, p^{\prime}- \\
\text { DDE } \\
(\mu g / L)\end{array}$ & $\begin{array}{c}\text { Pro- } \\
\text { meton } \\
(\mu g / L)\end{array}$ & $\begin{array}{l}\text { Pronamide } \\
(\mu \mathrm{g} / \mathrm{L})\end{array}$ & $\begin{array}{c}\text { Propa- } \\
\text { chlor } \\
(\mu \mathrm{g} / \mathrm{L})\end{array}$ \\
\hline RU-10-60-606 & 340427102302801 & $05-02-00$ & $<0.004$ & $<0.005$ & $<0.002$ & $<0.05$ & $<0.006$ & $<0.018$ & $<0.003$ & $<0.007$ \\
\hline RW-41-63-520 & 310238098104001 & $03-23-00$ & $<.004$ & $<.005$ & $<.002$ & $<.05$ & $<.006$ & $<.018$ & $<.003$ & $<.007$ \\
\hline SK-57-19-201 & 304323098421701 & $03-08-00$ & $<.004$ & $<.005$ & $<.002$ & $<.05$ & $<.006$ & $<.018$ & $<.003$ & $<.007$ \\
\hline SK-57-34-201 & 303001098485201 & 03-09-00 & $<.004$ & $<.005$ & $<.002$ & $<.05$ & $<.006$ & $<.018$ & $<.003$ & $<.007$ \\
\hline SP-23-17-417 & 334043101585701 & $05-10-00$ & $<.004$ & $<.005$ & $<.002$ & $<.05$ & $<.006$ & $<.018$ & $<.003$ & $<.007$ \\
\hline SP-23-18-107 & 334306101504101 & $05-10-00$ & $<.004$ & $<.005$ & $<.002$ & .410 & $<.006$ & $<.018$ & $<.003$ & $<.007$ \\
\hline SP-23-19-812 & 333955101404601 & $05-10-00$ & $<.004$ & $<.005$ & $<.002$ & $<.05$ & $<.006$ & $<.018$ & $<.003$ & $<.007$ \\
\hline SP-24-24-214 & 334419102045201 & $05-18-00$ & $<.004$ & $<.005$ & $<.002$ & $<.05$ & $<.006$ & $<.018$ & $<.003$ & $<.007$ \\
\hline SR-23-41-602 & 331933101543701 & $05-15-00$ & $<.004$ & $<.005$ & $<.002$ & $<.05$ & $<.006$ & $<.018$ & $<.003$ & $<.007$ \\
\hline SS-42-52-504 & 311202099321401 & $03-01-00$ & $<.004$ & $<.005$ & $<.002$ & $<.05$ & $<.006$ & $<.018$ & $<.003$ & $<.007$ \\
\hline ST-40-39-304 & 312745097083301 & $03-23-00$ & $<.004$ & $<.005$ & $<.002$ & $<.05$ & $<.006$ & $<.018$ & $<.003$ & $<.007$ \\
\hline SY-27-39-505 & 322555102105501 & $05-24-00$ & $<.004$ & $<.005$ & $<.002$ & $<.05$ & $<.006$ & $<.018$ & $<.003$ & $<.007$ \\
\hline SY-28-42-803 & 321619101495001 & $05-23-00$ & $<.004$ & $<.005$ & $<.002$ & $<.05$ & $<.006$ & $<.018$ & $<.003$ & $<.007$ \\
\hline SY-28-50-908 & 320816101474601 & $05-23-00$ & $<.004$ & $<.005$ & $<.002$ & $<.05$ & $<.006$ & .013 & $<.003$ & $<.007$ \\
\hline TB-76-03-605 & 285657100373801 & $03-28-00$ & $<.004$ & $<.005$ & $<.002$ & $<.05$ & $<.006$ & $<.018$ & $<.003$ & $<.007$ \\
\hline TJ-27-63-712 & 320143102123101 & $12-20-00$ & $<.01$ & $<.006$ & $<.011$ & $<.09$ & $<.0025$ & $<.015$ & $<.0041$ & $<.01$ \\
\hline TJ-28-57-702 & 320221101594901 & $05-25-00$ & $<.004$ & $<.005$ & $<.002$ & $<.05$ & $<.006$ & $<.018$ & $<.003$ & $<.007$ \\
\hline TJ-28-57-903 & 320010101523701 & $05-23-00$ & $<.004$ & $<.005$ & $<.002$ & $<.05$ & $<.006$ & $<.018$ & $<.003$ & $<.007$ \\
\hline TJ-45-07-407 & 315643102131101 & $05-24-00$ & $<.004$ & $<.005$ & $<.002$ & $<.05$ & $<.006$ & $<.018$ & $<.003$ & $<.007$ \\
\hline TJ-45-07-606 & 315702102075401 & $05-25-00$ & $<.004$ & $<.005$ & $<.002$ & $<.05$ & $<.006$ & .017 & $<.003$ & $<.007$ \\
\hline TS-60-36-510 & 302701095331201 & 04-20-00 & $<.004$ & $<.005$ & $<.002$ & $<.05$ & $<.006$ & $<.018$ & $<.003$ & $<.007$ \\
\hline TW-22-02-714 & 335416100502101 & $05-05-00$ & $<.004$ & $<.005$ & $<.002$ & $<.05$ & $<.006$ & $<.018$ & $<.003$ & $<.007$ \\
\hline TW-22-02-716 & 335449100513101 & $12-19-00$ & $<.01$ & $<.006$ & $<.011$ & $<.09$ & $<.0025$ & $<.015$ & $<.0041$ & $<.01$ \\
\hline UJ-62-49-712 & 300906093585201 & $04-28-00$ & $<.004$ & $<.005$ & $<.002$ & $<.05$ & $<.006$ & $<.018$ & $<.003$ & $<.007$ \\
\hline UK-31-05-501 & 325545098255501 & $03-22-00$ & $<.004$ & $<.005$ & $<.002$ & $<.05$ & $<.006$ & .022 & $<.003$ & $<.007$ \\
\hline UK-31-24-501 & 324210098034701 & $03-22-00$ & $<.004$ & $<.005$ & $<.002$ & $<.05$ & $<.006$ & $<.018$ & $<.003$ & $<.007$ \\
\hline UL-35-48-202 & 322207094034501 & $04-25-00$ & $<.004$ & $<.005$ & $<.002$ & $<.05$ & $<.006$ & $<.018$ & $<.003$ & $<.007$ \\
\hline UP-32-10-604 & 324926097455001 & 02-09-00 & $<.004$ & $<.005$ & $<.002$ & $<.05$ & $<.006$ & $<.018$ & $<.003$ & $<.007$ \\
\hline UP-32-11-103 & 325005097440101 & $02-09-00$ & $<.004$ & $<.005$ & $<.002$ & $<.05$ & $<.006$ & $<.018$ & $<.003$ & $<.007$ \\
\hline UP-32-11-709 & 324649097442001 & 02-09-00 & $<.004$ & $<.005$ & $<.002$ & $<.05$ & $<.006$ & .041 & $<.003$ & $<.007$ \\
\hline
\end{tabular}


Table 5. Concentrations of soluble pesticides in public water-supply wells sampled in Texas, November 1999-January 2001-Continued

\begin{tabular}{|c|c|c|c|c|c|c|c|c|c|c|}
\hline Local identifier & $\begin{array}{l}\text { Station } \\
\text { number }\end{array}$ & Date & $\begin{array}{l}\text { Pendi- } \\
\text { methalin } \\
(\mu \mathrm{g} / \mathrm{L})\end{array}$ & $\begin{array}{l}\text { Perme- } \\
\text { thrin } \\
(\mu \mathrm{g} / \mathrm{L})\end{array}$ & $\begin{array}{c}\text { Phorate } \\
(\mu \mathrm{g} / \mathrm{L})\end{array}$ & $\begin{array}{c}\text { Picloram } \\
(\mu \mathrm{g} / \mathrm{L})\end{array}$ & $\begin{array}{c}p, p^{\prime}- \\
\text { DDE } \\
(\mu \mathrm{g} / L)\end{array}$ & $\begin{array}{c}\text { Pro- } \\
\text { meton } \\
(\mu \mathrm{g} / L)\end{array}$ & $\begin{array}{l}\text { Pronamide } \\
(\mu \mathrm{g} / \mathrm{L})\end{array}$ & $\begin{array}{c}\text { Propa- } \\
\text { chlor } \\
(\mu \mathrm{g} / \mathrm{L})\end{array}$ \\
\hline UP-32-11-801 & 324548097411701 & $02-10-00$ & $<0.004$ & $<0.005$ & $<0.002$ & $<0.05$ & $<0.006$ & $<0.018$ & $<0.003$ & $<0.007$ \\
\hline UP-32-28-403 & 323356097361801 & $04-10-00$ & $<.004$ & $<.005$ & $<.002$ & $<.05$ & $<.006$ & $<.018$ & $<.003$ & $<.007$ \\
\hline US-53-08-601 & 305723102015801 & $03-14-00$ & $<.004$ & $<.005$ & $<.002$ & $<.05$ & $<.006$ & $<.018$ & $<.003$ & $<.007$ \\
\hline US-54-18-503 & 304131101492401 & $03-14-00$ & $<.004$ & $<.005$ & $<.002$ & $<.05$ & $<.006$ & $<.018$ & $<.003$ & $<.007$ \\
\hline UZ-44-37-505 & 312656101265101 & $01-13-00$ & $<.004$ & $<.005$ & $<.002$ & $<.05$ & $<.006$ & $<.018$ & $<.003$ & $<.007$ \\
\hline WB-16-18-704 & 333942094503001 & $04-26-00$ & $<.004$ & $<.005$ & $<.002$ & $<.05$ & $<.006$ & $<.018$ & $<.003$ & $<.007$ \\
\hline WD-46-46-210 & 312041103174201 & $01-11-00$ & $<.004$ & $<.005$ & $<.002$ & $<.05$ & $<.006$ & $<.018$ & $<.003$ & $<.007$ \\
\hline WK-39-51-801 & 310956096402901 & $04-20-00$ & $<.004$ & $<.005$ & $<.002$ & $<.05$ & $<.006$ & $<.018$ & $<.003$ & $<.007$ \\
\hline WP-43-31-211 & 313551100110801 & $04-25-00$ & $<.004$ & $<.005$ & $<.002$ & $<.05$ & $<.006$ & .066 & $<.003$ & $<.007$ \\
\hline XT-11-26-923 & 343046101463701 & $05-01-00$ & $<.004$ & $<.005$ & $<.002$ & $<.05$ & $<.006$ & $<.018$ & $<.003$ & $<.007$ \\
\hline XU-32-04-604 & 325525097304801 & $01-05-00$ & $<.004$ & $<.005$ & $<.002$ & $<.05$ & $<.006$ & $<.018$ & $<.003$ & $<.007$ \\
\hline XU-32-04-605 & 325525097304802 & $01-05-00$ & $<.004$ & $<.005$ & $<.002$ & $<.05$ & $<.006$ & $<.018$ & $<.003$ & $<.007$ \\
\hline XU-32-05-805 & 325335097272401 & 04-03-00 & $<.004$ & $<.005$ & $<.002$ & $<.05$ & $<.006$ & $<.018$ & $<.003$ & $<.007$ \\
\hline XU-32-12-307 & 325101097313101 & $03-23-00$ & $<.004$ & $<.005$ & $<.002$ & $<.05$ & $<.006$ & $<.018$ & $<.003$ & $<.007$ \\
\hline XU-32-13-405 & 324901097284701 & $01-24-00$ & $<.004$ & $<.005$ & $<.002$ & $<.05$ & $<.006$ & $<.018$ & $<.003$ & $<.007$ \\
\hline XU-32-13-503 & 324816097272401 & $01-24-00$ & $<.004$ & $<.005$ & $<.002$ & $<.05$ & $<.006$ & $<.018$ & $<.003$ & $<.007$ \\
\hline XU-32-23-701 & 323911097131001 & 03-20-00 & $<.004$ & $<.005$ & $<.002$ & $<.05$ & $<.006$ & $<.018$ & $<.003$ & $<.007$ \\
\hline XY-24-54-606 & 331112102160201 & $05-17-00$ & $<.004$ & $<.005$ & $<.002$ & $<.05$ & $<.006$ & $<.018$ & $<.003$ & $<.007$ \\
\hline YB-43-19-701 & 313943100425301 & $01-14-00$ & $<.004$ & $<.005$ & $<.002$ & $<.05$ & $<.006$ & .042 & $<.003$ & $<.007$ \\
\hline YB-43-27-201 & 313638100400201 & $01-14-00$ & $<.004$ & $<.005$ & $<.002$ & $<.05$ & $<.006$ & $<.018$ & $<.003$ & $<.007$ \\
\hline
\end{tabular}


Table 5. Concentrations of soluble pesticides in public water-supply wells sampled in Texas, November 1999-January 2001-Continued

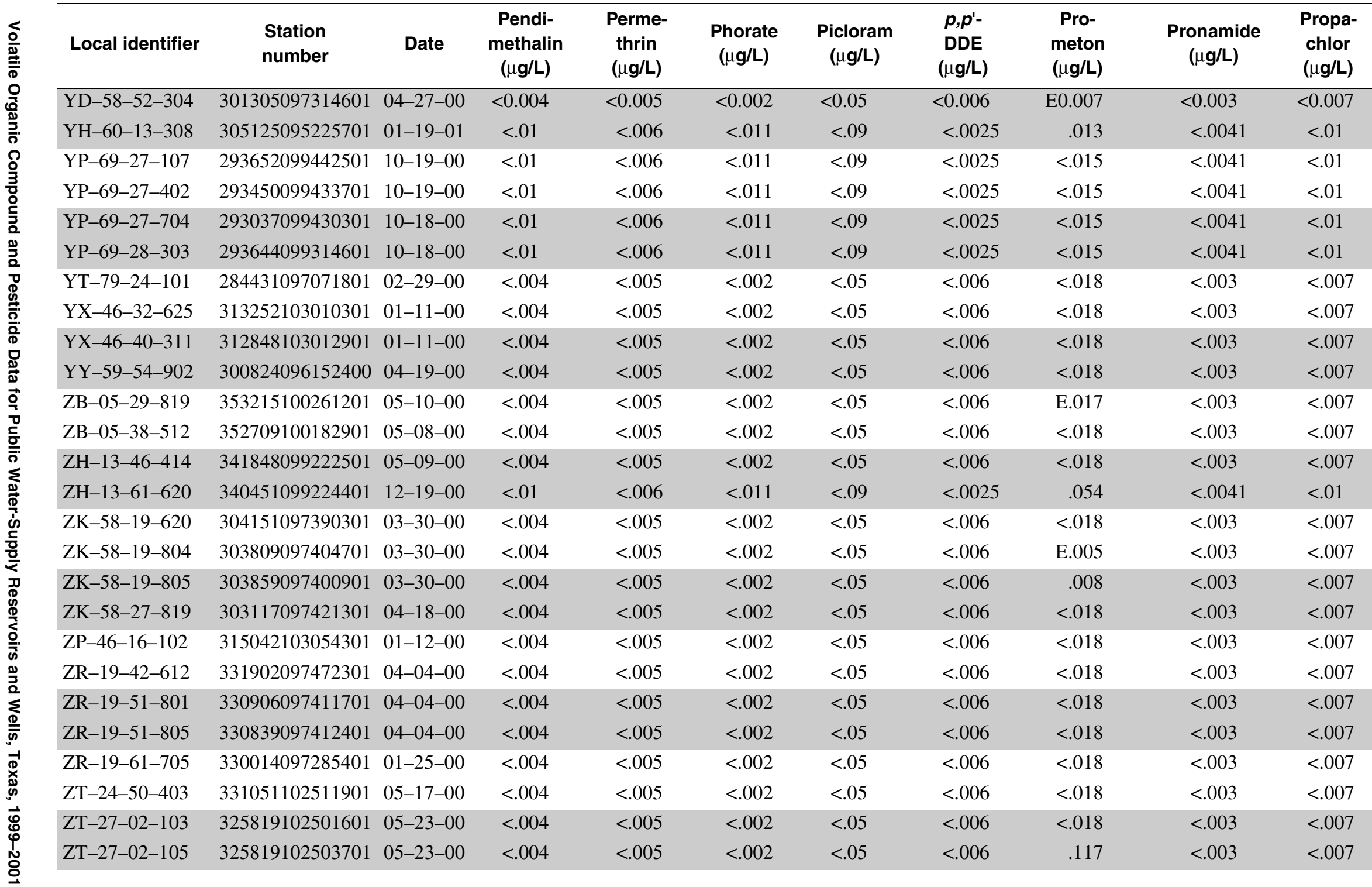


Table 5. Concentrations of soluble pesticides in public water-supply wells sampled in Texas, November 1999-January 2001-Continued

\begin{tabular}{|c|c|c|c|c|c|c|c|c|c|c|}
\hline Local identifier & $\begin{array}{l}\text { Station } \\
\text { number }\end{array}$ & Date & $\begin{array}{c}\text { Propanil } \\
(\mu \mathrm{g} / \mathrm{L})\end{array}$ & $\begin{array}{l}\text { Propargite } \\
(\mu \mathrm{g} / \mathrm{L})\end{array}$ & $\begin{array}{c}\text { Propham } \\
(\mu \mathrm{g} / \mathrm{L})\end{array}$ & $\begin{array}{l}\text { Propoxur } \\
(\mu \mathrm{g} / \mathrm{L})\end{array}$ & $\begin{array}{l}\text { Silvex } \\
(\mu \mathrm{g} / \mathrm{L})\end{array}$ & $\begin{array}{c}\text { Simazine } \\
(\mu \mathrm{g} / \mathrm{L})\end{array}$ & $\begin{array}{c}2,4,5-T \\
(\mu \mathrm{g} / \mathrm{L})\end{array}$ & $\begin{array}{l}\text { Tebuthi- } \\
\text { uron } \\
(\mu \mathbf{g} / L)\end{array}$ \\
\hline $\mathrm{AB}-27-37-103$ & 322805102283801 & $05-17-00$ & $<0.004$ & $<0.013$ & $<0.035$ & $<0.08$ & $<0.06$ & $<0.005$ & $<0.04$ & $<0.01$ \\
\hline AB-27-37-105 & 322826102280101 & $05-17-00$ & $<.004$ & $<.013$ & $<.035$ & $<.08$ & $<.06$ & $<.005$ & $<.04$ & $<.01$ \\
\hline AK-06-52-506 & 351024101334401 & 05-04-00 & $<.004$ & $<.013$ & $<.035$ & $<.08$ & $<.06$ & $<.005$ & $<.04$ & $<.01$ \\
\hline AP-59-62-403 & 300259096195001 & 04-03-00 & $<.004$ & $<.013$ & $<.035$ & $<.08$ & $<.06$ & $<.005$ & $<.04$ & $<.01$ \\
\hline AP-66-16-407 & 294903096061401 & $04-21-00$ & $<.004$ & $<.013$ & $<.035$ & $<.08$ & $<.06$ & $<.005$ & $<.04$ & $<.01$ \\
\hline AR-10-51-408 & 341229102435401 & $05-01-00$ & $<.004$ & $<.013$ & $<.035$ & $<.08$ & $<.06$ & $<.005$ & $<.04$ & $<.01$ \\
\hline AR-24-09-604 & 335000102545701 & 05-09-00 & $<.004$ & $<.013$ & $<.035$ & $<.08$ & $<.06$ & $<.005$ & $<.04$ & $<.01$ \\
\hline AT-58-62-206 & 300646097193001 & $12-10-99$ & $<.004$ & $<.013$ & $<.035$ & $<.08$ & $<.06$ & $<.005$ & $<.04$ & $<.01$ \\
\hline AU-21-30-389 & 333612099163101 & $05-02-00$ & $<.004$ & $<.013$ & $<.035$ & $<.08$ & $<.06$ & $<.005$ & $<.04$ & $<.01$ \\
\hline AX-58-04-604 & 305718097314401 & $03-22-00$ & $<.004$ & $<.013$ & $<.035$ & $<.08$ & $<.06$ & $<.005$ & $<.04$ & $<.01$ \\
\hline AX-58-04-623 & 305624097321101 & $03-22-00$ & $<.004$ & $<.013$ & $<.035$ & $<.08$ & $<.06$ & $<.005$ & $<.04$ & $<.01$ \\
\hline AY-68-35-105 & 292842098425001 & $03-06-00$ & $<.004$ & $<.013$ & $<.035$ & $<.08$ & $<.06$ & $<.005$ & $<.04$ & $<.01$ \\
\hline AZ-57-45-612 & 301923098225401 & $04-18-00$ & $<.004$ & $<.013$ & $<.035$ & $<.08$ & $<.06$ & $<.005$ & $<.04$ & $<.01$ \\
\hline AZ-57-45-811 & 301633098252901 & 03-29-00 & $<.004$ & $<.013$ & $<.035$ & $<.08$ & $<.06$ & $<.005$ & $<.04$ & $<.01$ \\
\hline BD-16-40-709 & 332301094061701 & $01-18-01$ & $<.011$ & $<.023$ & $<.09$ & $<.12$ & $<.025$ & $<.011$ & $<.04$ & $<.016$ \\
\hline BK-73-47-504 & 291911103124301 & $12-28-00$ & $<.011$ & $<.023$ & $<.09$ & $<.12$ & $<.025$ & $<.011$ & $<.04$ & $<.016$ \\
\hline BK-73-52-905 & 290819103312601 & $12-28-00$ & $<.011$ & $<.023$ & $<.09$ & $<.12$ & $<.025$ & $<.011$ & $<.04$ & $<.016$ \\
\hline BL-11-38-334 & 342841101155301 & $05-01-00$ & $<.004$ & $<.013$ & $<.035$ & $<.08$ & $<.06$ & $<.005$ & $<.04$ & $<.01$ \\
\hline BL-11-48-507 & 341919101035201 & $05-02-00$ & $<.004$ & $<.013$ & $<.035$ & $<.08$ & $<.06$ & $<.005$ & $<.04$ & $<.01$ \\
\hline BL-12-41-204 & 342109100570301 & $05-02-00$ & $<.004$ & $<.013$ & $<.035$ & $<.08$ & $<.06$ & $<.005$ & $<.04$ & $<.01$ \\
\hline BR-41-01-244 & 315849098552101 & $04-05-00$ & $<.004$ & $<.013$ & $<.035$ & $<.08$ & $<.06$ & $<.005$ & $<.04$ & $<.01$ \\
\hline BT-57-14-403 & 304939098202401 & $03-08-00$ & $<.004$ & $<.013$ & $<.035$ & $<.08$ & $<.06$ & $<.005$ & $<.04$ & $<.01$ \\
\hline BT-57-14-404 & 304927098211201 & $03-08-00$ & $<.004$ & $<.013$ & $<.035$ & $<.08$ & $<.06$ & $<.005$ & $<.04$ & $<.01$ \\
\hline BT-57-21-607 & 304125098224001 & $03-28-00$ & $<.004$ & $<.013$ & $<.035$ & $<.08$ & $<.06$ & $<.005$ & $<.04$ & $<.01$ \\
\hline BT-57-21-608 & 304121098224201 & $03-28-00$ & $<.004$ & $<.013$ & $<.035$ & $<.08$ & $<.06$ & $<.005$ & $<.04$ & $<.01$ \\
\hline BT-57-23-110 & 304406098130801 & 03-29-00 & $<.004$ & $<.013$ & $<.035$ & $<.08$ & $<.06$ & E.004 & $<.04$ & $<.01$ \\
\hline BT-57-30-101 & 303548098211701 & $03-01-00$ & $<.004$ & $<.013$ & $<.035$ & $<.08$ & $<.06$ & $<.005$ & $<.04$ & $<.01$ \\
\hline BU-67-10-910 & 294528097464401 & $03-21-00$ & $<.004$ & $<.013$ & $<1.33$ & $<.08$ & $<.06$ & .085 & $<.04$ & $<.01$ \\
\hline
\end{tabular}


Table 5. Concentrations of soluble pesticides in public water-supply wells sampled in Texas, November 1999-January 2001-Continued

\begin{tabular}{|c|c|c|c|c|c|c|c|c|c|c|}
\hline Local identifier & $\begin{array}{l}\text { Station } \\
\text { number }\end{array}$ & Date & $\begin{array}{l}\text { Propanil } \\
(\mu \mathrm{g} / \mathrm{L})\end{array}$ & $\begin{array}{c}\text { Propargite } \\
(\mu \mathrm{g} / \mathrm{L})\end{array}$ & $\begin{array}{c}\text { Propham } \\
\text { ( } \mu \mathrm{g} / \mathrm{L})\end{array}$ & $\begin{array}{l}\text { Propoxur } \\
(\mu \mathrm{g} / \mathrm{L})\end{array}$ & $\begin{array}{l}\text { Silvex } \\
(\mu g / L)\end{array}$ & $\begin{array}{c}\text { Simazine } \\
(\mu \mathrm{g} / \mathrm{L})\end{array}$ & $\begin{array}{c}2,4,5-T \\
(\mu \mathrm{g} / \mathrm{L})\end{array}$ & $\begin{array}{c}\text { Tebuthi- } \\
\text { uron } \\
(\mu \mathrm{g} / \mathrm{L})\end{array}$ \\
\hline $\mathrm{BX}-30-55-936$ & 320939099093801 & $12-18-00$ & $<0.011$ & $<0.023$ & $<0.09$ & $<0.12$ & $<0.025$ & $<0.011$ & $<0.04$ & $<0.016$ \\
\hline DA-06-28-202 & 353704101343701 & 05-03-00 & $<.004$ & $<.013$ & $<.035$ & $<.08$ & $<.06$ & $<.005$ & $<.04$ & $<.01$ \\
\hline DL-14-62-604 & 340406098160701 & $05-10-00$ & $<.004$ & $<.013$ & $<.035$ & $<.08$ & $<.06$ & $<.005$ & $<.04$ & $<.01$ \\
\hline DP-24-18-308 & 334352102450601 & 05-09-00 & $<.004$ & $<.013$ & $<.035$ & $<.08$ & $<.06$ & $<.005$ & $<.04$ & $<.01$ \\
\hline DP-25-24-304 & 334245103011001 & $05-08-00$ & $<.004$ & $<.013$ & $<.035$ & $<.08$ & $<.06$ & $<.005$ & $<.04$ & $<.01$ \\
\hline DU-12-06-609 & 345658100155501 & $05-08-00$ & $<.004$ & $<.013$ & $<.035$ & $<.08$ & $<.06$ & $<.005$ & $<.04$ & $<.01$ \\
\hline DX-68-05-621 & 295520098242301 & $03-22-00$ & $<.004$ & $<.013$ & $<.035$ & $<.08$ & $<.06$ & $<.005$ & $<.04$ & $<.01$ \\
\hline DX-68-06-405 & 295528098222301 & $03-22-00$ & $<.004$ & $<.013$ & $<.035$ & $<.08$ & $<.06$ & $<.005$ & $<.04$ & $<.01$ \\
\hline DY-41-13-307 & 315105098241401 & $04-05-00$ & $<.004$ & $<.013$ & $<.035$ & $<.08$ & $<.06$ & $<.005$ & $<.04$ & $<.01$ \\
\hline $\mathrm{HH}-45-35-505$ & 312715102402201 & $01-12-00$ & $<.004$ & $<.013$ & $<.035$ & $<.08$ & $<.06$ & $<.005$ & $<.04$ & $<.01$ \\
\hline HP-12-62-802 & 340210100184901 & $05-05-00$ & $<.004$ & $<.013$ & -- & -- & $<.06$ & $<.005$ & $<.04$ & $<.01$ \\
\hline HS-28-09-404 & 324810101591201 & $05-16-00$ & $<.004$ & $<.013$ & $<.035$ & $<.08$ & $<.06$ & $<.005$ & $<.04$ & $<.01$ \\
\hline HT-07-63-204 & 350607102103401 & $05-03-00$ & $<.004$ & $<.013$ & $<.035$ & $<.08$ & $<.06$ & $<.005$ & $<.04$ & $<.01$ \\
\hline HZ-77-34-606 & 282608099455301 & $03-28-00$ & $<.004$ & $<.013$ & $<.035$ & $<.08$ & $<.06$ & $<.005$ & $<.04$ & $<.01$ \\
\hline JA-05-57-613 & 350254100535501 & 05-04-00 & $<.004$ & $<.013$ & $<.035$ & $<.08$ & $<.06$ & $<.005$ & $<.04$ & $<.01$ \\
\hline JA-12-12-404 & 344910100360401 & $05-02-00$ & $<.004$ & $<.013$ & $<.035$ & $<.08$ & $<.06$ & $<.005$ & $<.04$ & $<.01$ \\
\hline JH-45-05-924 & 315245102240201 & $05-25-00$ & $<.004$ & $<.013$ & $<.035$ & $<.08$ & $<.06$ & $<.005$ & $<.04$ & .067 \\
\hline JL-49-04-116 & 315757106370201 & $01-06-00$ & $<.004$ & $<.013$ & $<.035$ & $<.08$ & $<.06$ & $<.005$ & $<.04$ & $<.01$ \\
\hline JL-49-04-423 & 315708106362301 & 01-03-00 & $<.004$ & $<.013$ & $<.035$ & $<.08$ & $<.06$ & $<.005$ & $<.04$ & $<.01$ \\
\hline JL-49-04-428 & 315517106361401 & 01-06-00 & $<.004$ & $<.013$ & $<.035$ & $<.08$ & $<.06$ & $<.005$ & $<.04$ & $<.01$ \\
\hline JL-49-04-492 & 315623106360601 & $01-06-00$ & $<.004$ & $<.03$ & $<.035$ & $<.08$ & $<.06$ & $<.005$ & $<.04$ & $<.01$ \\
\hline JL-49-22-626 & 314145106163601 & $01-04-00$ & $<.004$ & $<.04$ & $<.035$ & $<.08$ & $<.06$ & $<.005$ & $<.04$ & $<.01$ \\
\hline JL-49-24-423 & 314107106063301 & $01-07-00$ & $<.004$ & $<.04$ & $<.035$ & $<.08$ & $<.06$ & $<.005$ & $<.04$ & $<.01$ \\
\hline JL-49-40-502 & 312648106044701 & 01-04-00 & $<.004$ & $<.013$ & $<.035$ & $<.08$ & $<.06$ & $<.005$ & $<.04$ & $<.01$ \\
\hline JY-65-26-520 & 293314095474702 & 04-03-00 & $<.004$ & $<.013$ & $<.035$ & $<.08$ & $<.06$ & $<.005$ & $<.04$ & $<.01$ \\
\hline KD-27-01-612 & 325523102542701 & $05-22-00$ & $<.004$ & $<.013$ & $<.035$ & $<.08$ & $<.06$ & $<.005$ & $<.04$ & $<.01$ \\
\hline KK-57-42-709 & 301545098502801 & 07-06-00 & $<.004$ & $<.013$ & $<.035$ & $<.08$ & $<.06$ & $<.005$ & $<.04$ & $<.01$ \\
\hline $\mathrm{KK}-57-50-325$ & 301357098471901 & $03-28-00$ & $<.004$ & $<.013$ & $<.035$ & $<.08$ & $<.06$ & .005 & $<.04$ & $<.01$ \\
\hline KK-57-51-303 & 301411098383101 & $03-21-00$ & $<.004$ & $<.013$ & $<.035$ & $<.08$ & $<.06$ & $<.005$ & $<.04$ & $<.01$ \\
\hline KK-57-52-107 & 301430098363401 & $03-21-00$ & $<.004$ & $<.013$ & $<.035$ & $<.08$ & $<.06$ & $<.005$ & $<.04$ & $<.01$ \\
\hline
\end{tabular}


Table 5. Concentrations of soluble pesticides in public water-supply wells sampled in Texas, November 1999-January 2001-Continued

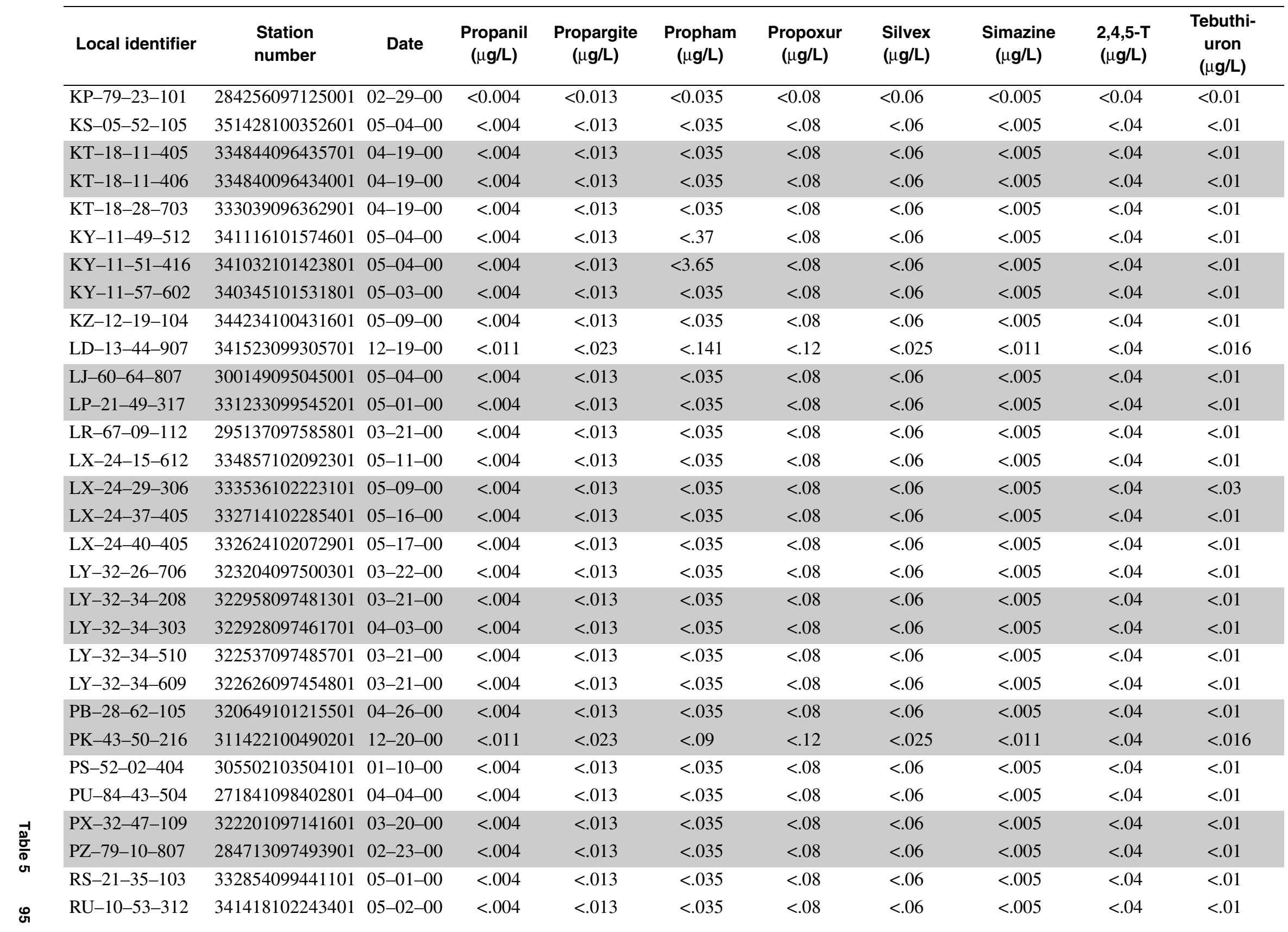


Table 5. Concentrations of soluble pesticides in public water-supply wells sampled in Texas, November 1999-January 2001-Continued

\begin{tabular}{|c|c|c|c|c|c|c|c|c|c|c|}
\hline Local identifier & $\begin{array}{l}\text { Station } \\
\text { number }\end{array}$ & Date & $\begin{array}{l}\text { Propanil } \\
(\mu \mathrm{g} / \mathrm{L})\end{array}$ & $\begin{array}{l}\text { Propargite } \\
(\mu \mathrm{g} / \mathrm{L})\end{array}$ & $\begin{array}{l}\text { Propham } \\
(\mu \mathrm{g} / \mathrm{L})\end{array}$ & $\begin{array}{l}\text { Propoxur } \\
(\mu \mathrm{g} / \mathrm{L})\end{array}$ & $\begin{array}{l}\text { Silvex } \\
(\mu \mathrm{g} / \mathrm{L})\end{array}$ & $\begin{array}{c}\text { Simazine } \\
(\mu \mathrm{g} / \mathrm{L})\end{array}$ & $\begin{array}{c}2,4,5-T \\
(\mu \mathrm{g} / \mathrm{L})\end{array}$ & $\begin{array}{c}\text { Tebuthi- } \\
\text { uron } \\
(\mu \mathrm{g} / \mathrm{L})\end{array}$ \\
\hline RU-10-60-606 & 340427102302801 & $05-02-00$ & $<0.004$ & $<0.013$ & $<0.035$ & $<0.08$ & $<0.06$ & $<0.005$ & $<0.04$ & $<0.01$ \\
\hline$R W-41-63-520$ & 310238098104001 & $03-23-00$ & $<.004$ & $<.013$ & $<.035$ & $<.08$ & $<.06$ & $<.005$ & $<.04$ & $<.01$ \\
\hline SK-57-19-201 & 304323098421701 & $03-08-00$ & $<.004$ & $<.013$ & $<.035$ & $<.08$ & $<.06$ & $<.005$ & $<.04$ & $<.01$ \\
\hline SK-57-34-201 & 303001098485201 & 03-09-00 & $<.004$ & $<.013$ & $<.035$ & $<.08$ & $<.06$ & $<.005$ & $<.04$ & $<.01$ \\
\hline SP-23-17-417 & 334043101585701 & $05-10-00$ & $<.004$ & $<.013$ & $<.035$ & $<.08$ & $<.06$ & $<.005$ & $<.04$ & $<.01$ \\
\hline SP-23-18-107 & 334306101504101 & $05-10-00$ & $<.004$ & $<.013$ & $<.14$ & $<.08$ & $<.06$ & $<.005$ & $<.04$ & $<.01$ \\
\hline SP-23-19-812 & 333955101404601 & $05-10-00$ & $<.004$ & $<.013$ & $<.035$ & $<.08$ & $<.06$ & $<.005$ & $<.04$ & $<.01$ \\
\hline SP-24-24-214 & 334419102045201 & $05-18-00$ & $<.004$ & $<.013$ & $<.035$ & $<.08$ & $<.06$ & $<.005$ & $<.04$ & $<.01$ \\
\hline SR-23-41-602 & 331933101543701 & $05-15-00$ & $<.004$ & $<.013$ & $<.035$ & $<.08$ & $<.06$ & $<.005$ & $<.04$ & $<.01$ \\
\hline SS-42-52-504 & 311202099321401 & $03-01-00$ & $<.004$ & $<.013$ & $<.035$ & $<.08$ & $<.06$ & $<.005$ & $<.04$ & $<.01$ \\
\hline ST-40-39-304 & 312745097083301 & $03-23-00$ & $<.004$ & $<.013$ & $<.035$ & $<.08$ & $<.06$ & $<.005$ & $<.04$ & $<.01$ \\
\hline$S Y-27-39-505$ & 322555102105501 & $05-24-00$ & $<.004$ & $<.013$ & $<.035$ & $<.08$ & $<.06$ & $<.005$ & $<.04$ & $<.01$ \\
\hline SY $-28-42-803$ & 321619101495001 & $05-23-00$ & $<.004$ & $<.013$ & $<.035$ & $<.08$ & $<.06$ & $<.005$ & $<.04$ & $<.01$ \\
\hline SY-28-50-908 & 320816101474601 & $05-23-00$ & $<.004$ & $<.013$ & $<.035$ & $<.21$ & $<.06$ & $<.005$ & $<.04$ & $<.01$ \\
\hline TB-76-03-605 & 285657100373801 & $03-28-00$ & $<.004$ & $<.013$ & $<.035$ & $<.08$ & $<.06$ & $<.005$ & $<.04$ & $<.01$ \\
\hline TJ-27-63-712 & 320143102123101 & $12-20-00$ & $<.011$ & $<.023$ & $<.09$ & $<.12$ & $<.025$ & $<.011$ & $<.04$ & $<.016$ \\
\hline TJ-28-57-702 & 320221101594901 & $05-25-00$ & $<.004$ & $<.013$ & $<.035$ & $<.08$ & $<.06$ & $<.005$ & $<.04$ & $<.01$ \\
\hline TJ-28-57-903 & 320010101523701 & $05-23-00$ & $<.004$ & $<.013$ & $<.035$ & $<.08$ & $<.06$ & $<.005$ & $<.04$ & $<.01$ \\
\hline TJ-45-07-407 & 315643102131101 & $05-24-00$ & $<.004$ & $<.013$ & $<.035$ & $<.08$ & $<.06$ & $<.005$ & $<.04$ & $<.01$ \\
\hline TJ-45-07-606 & 315702102075401 & $05-25-00$ & $<.004$ & $<.013$ & $<.035$ & $<.16$ & $<.06$ & $<.005$ & $<.04$ & $<.01$ \\
\hline TS-60-36-510 & 302701095331201 & $04-20-00$ & $<.004$ & $<.013$ & $<.035$ & $<.08$ & $<.06$ & $<.005$ & $<.04$ & $<.01$ \\
\hline TW-22-02-714 & 335416100502101 & $05-05-00$ & $<.004$ & $<.013$ & $<.035$ & $<.08$ & $<.06$ & $<.005$ & $<.04$ & $<.01$ \\
\hline TW-22-02-716 & 335449100513101 & $12-19-00$ & $<.011$ & $<.023$ & $<.09$ & $<.12$ & $<.025$ & $<.011$ & $<.04$ & $<.016$ \\
\hline UJ-62-49-712 & 300906093585201 & $04-28-00$ & $<.004$ & $<.013$ & $<.035$ & $<.08$ & $<.06$ & $<.005$ & $<.04$ & $<.01$ \\
\hline UK-31-05-501 & 325545098255501 & $03-22-00$ & $<.004$ & $<.013$ & $<.035$ & $<.08$ & $<.06$ & $<.005$ & $<.04$ & $<.01$ \\
\hline $\mathrm{UK}-31-24-501$ & 324210098034701 & $03-22-00$ & $<.004$ & $<.013$ & $<.035$ & $<.08$ & $<.06$ & $<.005$ & $<.04$ & $<.01$ \\
\hline UL-35-48-202 & 322207094034501 & $04-25-00$ & $<.004$ & $<.013$ & $<.035$ & $<.08$ & $<.06$ & $<.005$ & $<.04$ & $<.01$ \\
\hline UP-32-10-604 & 324926097455001 & 02-09-00 & $<.004$ & $<.013$ & $<.035$ & $<.08$ & $<.06$ & $<.005$ & $<.04$ & $<.01$ \\
\hline UP-32-11-103 & 325005097440101 & 02-09-00 & $<.004$ & $<.013$ & $<.035$ & $<.08$ & $<.06$ & $<.005$ & $<.04$ & $<.01$ \\
\hline UP-32-11-709 & 324649097442001 & 02-09-00 & $<.004$ & $<.013$ & $<.035$ & $<.08$ & $<.06$ & $<.005$ & $<.04$ & $<.01$ \\
\hline
\end{tabular}


Table 5. Concentrations of soluble pesticides in public water-supply wells sampled in Texas, November 1999-January 2001-Continued

\begin{tabular}{|c|c|c|c|c|c|c|c|c|c|c|}
\hline Local identifier & $\begin{array}{l}\text { Station } \\
\text { number }\end{array}$ & Date & $\begin{array}{l}\text { Propanil } \\
(\mu \mathrm{g} / \mathrm{L})\end{array}$ & $\begin{array}{l}\text { Propargite } \\
(\mu \mathbf{g} / L)\end{array}$ & $\begin{array}{c}\text { Propham } \\
(\mu \mathrm{g} / \mathrm{L})\end{array}$ & $\begin{array}{l}\text { Propoxur } \\
(\mu \mathrm{g} / \mathrm{L})\end{array}$ & $\begin{array}{l}\text { Silvex } \\
(\mu \mathrm{g} / \mathrm{L})\end{array}$ & $\begin{array}{c}\text { Simazine } \\
(\mu \mathrm{g} / \mathrm{L})\end{array}$ & $\begin{array}{c}2,4,5-T \\
(\mu \mathrm{g} / \mathrm{L})\end{array}$ & $\begin{array}{l}\text { Tebuthi- } \\
\text { uron } \\
(\mu \mathrm{g} / \mathrm{L})\end{array}$ \\
\hline UP-32-11-801 & 324548097411701 & $02-10-00$ & $<0.004$ & $<0.013$ & $<0.035$ & $<0.08$ & $<0.06$ & $<0.005$ & $<0.04$ & $<0.01$ \\
\hline UP-32-28-403 & 323356097361801 & 04-10-00 & $<.004$ & $<.013$ & $<.035$ & $<.08$ & $<.06$ & $<.005$ & $<.04$ & $<.01$ \\
\hline US-53-08-601 & 305723102015801 & $03-14-00$ & $<.004$ & $<.013$ & $<.035$ & $<.08$ & $<.06$ & $<.005$ & $<.04$ & $<.01$ \\
\hline US-54-18-503 & 304131101492401 & $03-14-00$ & $<.004$ & $<.013$ & $<.035$ & $<.08$ & $<.06$ & $<.005$ & $<.04$ & .063 \\
\hline UZ-44-37-505 & 312656101265101 & 01-13-00 & $<.004$ & $<.013$ & $<.035$ & $<.08$ & $<.06$ & $<.005$ & $<.04$ & $<.01$ \\
\hline WB-16-18-704 & 333942094503001 & 04-26-00 & $<.004$ & $<.013$ & $<.035$ & $<.08$ & $<.06$ & $<.005$ & $<.04$ & $<.01$ \\
\hline WD-46-46-210 & 312041103174201 & $01-11-00$ & $<.004$ & $<.013$ & $<.1$ & $<.08$ & $<.06$ & $<.005$ & $<.04$ & $<.01$ \\
\hline WK-39-51-801 & 310956096402901 & 04-20-00 & $<.004$ & $<.013$ & $<.035$ & $<.08$ & $<.06$ & $<.005$ & $<.04$ & $<.01$ \\
\hline WP-43-31-211 & 313551100110801 & $04-25-00$ & $<.004$ & $<.013$ & $<.035$ & $<.08$ & $<.06$ & $<.005$ & $<.04$ & $<.01$ \\
\hline XT-11-26-923 & 343046101463701 & 05-01-00 & $<.004$ & $<.013$ & $<.035$ & $<.08$ & $<.06$ & $<.005$ & $<.04$ & $<.01$ \\
\hline XU-32-04-604 & 325525097304801 & $01-05-00$ & $<.004$ & $<.013$ & $<.035$ & $<.08$ & $<.06$ & $<.005$ & $<.04$ & $<.01$ \\
\hline XU-32-04-605 & 325525097304802 & $01-05-00$ & $<.004$ & $<.013$ & $<.035$ & $<.08$ & $<.06$ & $<.005$ & $<.04$ & $<.01$ \\
\hline XU-32-05-805 & 325335097272401 & 04-03-00 & $<.004$ & $<.013$ & $<.035$ & $<.08$ & $<.06$ & $<.005$ & $<.04$ & $<.01$ \\
\hline XU-32-12-307 & 325101097313101 & $03-23-00$ & $<.004$ & $<.013$ & $<.035$ & $<.08$ & $<.06$ & $<.005$ & $<.04$ & $<.01$ \\
\hline XU-32-13-405 & 324901097284701 & $01-24-00$ & $<.004$ & $<.013$ & $<.035$ & $<.08$ & $<.06$ & $<.005$ & $<.04$ & $<.01$ \\
\hline XU-32-13-503 & 324816097272401 & $01-24-00$ & $<.004$ & $<.013$ & $<.035$ & $<.08$ & $<.06$ & $<.005$ & $<.04$ & $<.01$ \\
\hline XU-32-23-701 & 323911097131001 & $03-20-00$ & $<.004$ & $<.013$ & $<.035$ & $<.08$ & $<.06$ & $<.005$ & $<.04$ & $<.01$ \\
\hline$X Y-24-54-606$ & 331112102160201 & $05-17-00$ & $<.004$ & $<.013$ & $<.035$ & $<.08$ & $<.06$ & $<.005$ & $<.04$ & $<.01$ \\
\hline YB-43-19-701 & 313943100425301 & $01-14-00$ & $<.004$ & $<.013$ & $<.035$ & $<.08$ & $<.06$ & $<.005$ & $<.04$ & $<.01$ \\
\hline YB-43-27-201 & 313638100400201 & $01-14-00$ & $<.004$ & $<.013$ & $<.035$ & $<.08$ & $<.06$ & $<.005$ & $<.04$ & $<.01$ \\
\hline
\end{tabular}


Table 5. Concentrations of soluble pesticides in public water-supply wells sampled in Texas, November 1999-January 2001-Continued

\begin{tabular}{|c|c|c|c|c|c|c|c|c|c|c|}
\hline Local identifier & $\begin{array}{l}\text { Station } \\
\text { number }\end{array}$ & Date & $\begin{array}{l}\text { Propanil } \\
(\mu \mathrm{g} / \mathrm{L})\end{array}$ & $\begin{array}{l}\text { Propargite } \\
(\mu \mathrm{g} / \mathrm{L})\end{array}$ & $\begin{array}{l}\text { Propham } \\
(\mu \mathrm{g} / \mathrm{L})\end{array}$ & $\begin{array}{l}\text { Propoxur } \\
(\mu \mathrm{g} / \mathrm{L})\end{array}$ & $\begin{array}{l}\text { Silvex } \\
(\mu \mathrm{g} / \mathrm{L})\end{array}$ & $\begin{array}{c}\text { Simazine } \\
(\mu \mathrm{g} / \mathrm{L})\end{array}$ & $\begin{array}{c}2,4,5-T \\
(\mu \mathrm{g} / \mathrm{L})\end{array}$ & $\begin{array}{c}\text { Tebuthi- } \\
\text { uron } \\
(\mu \mathrm{g} / \mathrm{L})\end{array}$ \\
\hline YD-58-52-304 & 301305097314601 & $04-27-00$ & $<0.004$ & $<0.013$ & $<0.035$ & $<0.08$ & $<0.06$ & 0.013 & $<0.04$ & $<0.01$ \\
\hline YH-60-13-308 & 305125095225701 & $01-19-01$ & $<.011$ & $<.023$ & $<.14$ & $<.12$ & $<.025$ & .023 & $<.04$ & .021 \\
\hline YP-69-27-107 & 293652099442501 & $10-19-00$ & $<.011$ & $<.023$ & $<.09$ & $<.12$ & $<.025$ & $<.011$ & $<.04$ & $<.016$ \\
\hline YP-69-27-402 & 293450099433701 & $10-19-00$ & $<.011$ & $<.023$ & $<.09$ & $<.12$ & $<.025$ & $<.011$ & $<.04$ & $<.016$ \\
\hline YP-69-27-704 & 293037099430301 & $10-18-00$ & $<.011$ & $<.023$ & $<.09$ & $<.12$ & $<.025$ & $<.011$ & $<.04$ & $<.016$ \\
\hline YP-69-28-303 & 293644099314601 & $10-18-00$ & $<.011$ & $<.023$ & $<.09$ & $<.12$ & $<.025$ & $<.011$ & $<.04$ & $<.016$ \\
\hline YT-79-24-101 & 284431097071801 & 02-29-00 & $<.004$ & $<.013$ & $<.035$ & $<.08$ & $<.06$ & $<.005$ & $<.04$ & $<.01$ \\
\hline$Y X-46-32-625$ & 313252103010301 & $01-11-00$ & $<.004$ & $<.013$ & $<.035$ & $<.08$ & $<.06$ & $<.005$ & $<.04$ & $<.01$ \\
\hline$Y X-46-40-311$ & 312848103012901 & $01-11-00$ & $<.004$ & $<.013$ & $<.035$ & $<.08$ & $<.06$ & $<.005$ & $<.04$ & $<.01$ \\
\hline YY-59-54-902 & 300824096152400 & 04-19-00 & $<.004$ & $<.013$ & $<.035$ & $<.08$ & $<.06$ & $<.005$ & $<.04$ & $<.01$ \\
\hline ZB-05-29-819 & 353215100261201 & $05-10-00$ & $<.004$ & $<.013$ & $<.035$ & $<.08$ & $<.06$ & $<.005$ & $<.04$ & $<.01$ \\
\hline ZB-05-38-512 & 352709100182901 & $05-08-00$ & $<.004$ & $<.013$ & $<.035$ & $<.08$ & $<.06$ & $<.005$ & $<.04$ & $<.01$ \\
\hline $\mathrm{ZH}-13-46-414$ & 341848099222501 & 05-09-00 & $<.004$ & $<.013$ & $<.035$ & $<.08$ & $<.06$ & $<.005$ & $<.04$ & $<.01$ \\
\hline $\mathrm{ZH}-13-61-620$ & 340451099224401 & $12-19-00$ & $<.011$ & $<.023$ & $<.09$ & $<.194$ & $<.025$ & $<.011$ & $<.04$ & $<.016$ \\
\hline ZK-58-19-620 & 304151097390301 & $03-30-00$ & $<.004$ & $<.013$ & $<.035$ & $<.08$ & $<.06$ & .011 & $<.04$ & $<.01$ \\
\hline ZK-58-19-804 & 303809097404701 & $03-30-00$ & $<.004$ & $<.013$ & $<.12$ & $<.08$ & $<.06$ & .006 & $<.04$ & $<.01$ \\
\hline ZK-58-19-805 & 303859097400901 & $03-30-00$ & $<.004$ & $<.013$ & $<.14$ & $<.08$ & $<.06$ & .005 & $<.04$ & $<.01$ \\
\hline $\mathrm{ZK}-58-27-819$ & 303117097421301 & $04-18-00$ & $<.004$ & $<.013$ & $<.035$ & $<.08$ & $<.06$ & $<.005$ & $<.04$ & $<.01$ \\
\hline $\mathrm{ZP}-46-16-102$ & 315042103054301 & $01-12-00$ & $<.004$ & $<.013$ & $<.035$ & $<.08$ & $<.06$ & $<.005$ & $<.04$ & $<.01$ \\
\hline ZR-19-42-612 & 331902097472301 & 04-04-00 & $<.004$ & $<.013$ & $<.035$ & $<.08$ & $<.06$ & $<.005$ & $<.04$ & $<.01$ \\
\hline ZR-19-51-801 & 330906097411701 & 04-04-00 & $<.004$ & $<.013$ & $<.035$ & $<.08$ & $<.06$ & $<.005$ & $<.04$ & $<.01$ \\
\hline ZR-19-51-805 & 330839097412401 & 04-04-00 & $<.004$ & $<.013$ & $<.035$ & $<.08$ & $<.06$ & $<.005$ & $<.04$ & $<.01$ \\
\hline ZR-19-61-705 & 330014097285401 & $01-25-00$ & $<.004$ & $<.013$ & $<.035$ & $<.08$ & $<.06$ & $<.005$ & $<.04$ & $<.01$ \\
\hline ZT-24-50-403 & 331051102511901 & $05-17-00$ & $<.004$ & $<.013$ & $<.035$ & $<.08$ & $<.06$ & $<.005$ & $<.04$ & $<.01$ \\
\hline ZT-27-02-103 & 325819102501601 & $05-23-00$ & $<.004$ & $<.013$ & $<.035$ & $<.08$ & $<.06$ & $<.005$ & $<.04$ & $<.01$ \\
\hline ZT-27-02-105 & 325819102503701 & $05-23-00$ & $<.004$ & $<.013$ & $<.14$ & $<.31$ & $<.06$ & .032 & $<.04$ & .038 \\
\hline
\end{tabular}


Table 5. Concentrations of soluble pesticides in public water-supply wells sampled in Texas, November 1999-January 2001-Continued

\begin{tabular}{|c|c|c|c|c|c|c|c|c|}
\hline Local identifier & $\begin{array}{l}\text { Station } \\
\text { number }\end{array}$ & Date & $\begin{array}{c}\text { Terbacil } \\
(\mu \mathrm{g} / \mathrm{L})\end{array}$ & $\begin{array}{c}\text { Terbufos } \\
\text { ( } \mu \mathrm{g} / \mathrm{L})\end{array}$ & $\begin{array}{c}\text { Thiobencarb } \\
(\mu \mathrm{g} / \mathrm{L})\end{array}$ & $\begin{array}{c}\text { Triallate } \\
(\mu \mathrm{g} / \mathrm{L})\end{array}$ & $\begin{array}{l}\text { Triclopyr } \\
(\mu \mathrm{g} / \mathrm{L})\end{array}$ & $\begin{array}{c}\text { Trifluralin } \\
(\mu \mathrm{g} / L)\end{array}$ \\
\hline AB-27-37-105 & 322826102280101 & $05-17-00$ & $<.007$ & $<.013$ & $<.002$ & $<.001$ & $<.25$ & $<.002$ \\
\hline AP-59-62-403 & 300259096195001 & 04-03-00 & $<.007$ & $<.013$ & $<.002$ & $<.001$ & $<.25$ & $<.002$ \\
\hline AP-66-16-407 & 294903096061401 & $04-21-00$ & $<.007$ & $<.013$ & $<.002$ & $<.001$ & $<.25$ & $<.002$ \\
\hline AR-10-51-408 & 341229102435401 & $05-01-00$ & $<.007$ & $<.013$ & $<.002$ & $<.001$ & $<.25$ & $<.002$ \\
\hline AT-58-62-206 & 300646097193001 & $12-10-99$ & $<.007$ & $<.013$ & $<.002$ & $<.001$ & $<.25$ & $<.002$ \\
\hline AU-21-30-389 & 333612099163101 & $05-02-00$ & $<.007$ & $<.013$ & $<.002$ & $<.001$ & $<.25$ & $<.002$ \\
\hline$A X-58-04-604$ & 305718097314401 & $03-22-00$ & $<.007$ & $<.013$ & $<.002$ & $<.001$ & $<.25$ & $<.002$ \\
\hline $\mathrm{AX}-58-04-623$ & 305624097321101 & $03-22-00$ & $<.007$ & $<.013$ & $<.002$ & $<.001$ & $<.25$ & $<.002$ \\
\hline AY-68-35-105 & 292842098425001 & 03-06-00 & $<.007$ & $<.013$ & $<.002$ & $<.001$ & $<.25$ & $<.002$ \\
\hline BK-73-52-905 & 290819103312601 & $12-28-00$ & $<.034$ & $<.017$ & $<.0048$ & $<.0023$ & $<.07$ & $<.009$ \\
\hline BL-11-38-334 & 342841101155301 & $05-01-00$ & $<.007$ & $<.013$ & $<.002$ & $<.001$ & $<.25$ & $<.002$ \\
\hline BL-11-48-507 & 341919101035201 & $05-02-00$ & $<.007$ & $<.013$ & $<.002$ & $<.001$ & $<.25$ & $<.002$ \\
\hline BL-12-41-204 & 342109100570301 & $05-02-00$ & $<.007$ & $<.013$ & $<.002$ & $<.001$ & $<.25$ & $<.002$ \\
\hline BR-41-01-244 & 315849098552101 & 04-05-00 & $<.007$ & $<.013$ & $<.002$ & $<.001$ & $<.25$ & $<.002$ \\
\hline BT-57-14-403 & 304939098202401 & $03-08-00$ & $<.007$ & $<.013$ & $<.002$ & $<.001$ & $<.25$ & $<.002$ \\
\hline BT-57-14-404 & 304927098211201 & 03-08-00 & $<.007$ & $<.013$ & $<.002$ & $<.001$ & $<.25$ & $<.002$ \\
\hline BT-57-21-607 & 304125098224001 & $03-28-00$ & $<.007$ & $<.013$ & $<.002$ & $<.001$ & $<.25$ & $<.002$ \\
\hline BT-57-21-608 & 304121098224201 & $03-28-00$ & $<.007$ & $<.013$ & $<.002$ & $<.001$ & $<.25$ & $<.002$ \\
\hline BT-57-23-110 & 304406098130801 & 03-29-00 & $<.007$ & $<.013$ & $<.002$ & $<.001$ & $<.25$ & $<.002$ \\
\hline BT-57-30-101 & 303548098211701 & 03-01-00 & $<.007$ & $<.013$ & $<.002$ & $<.001$ & $<.25$ & $<.002$ \\
\hline
\end{tabular}


Table 5. Concentrations of soluble pesticides in public water-supply wells sampled in Texas, November 1999-January 2001-Continued

\begin{tabular}{|c|c|c|c|c|c|c|c|c|}
\hline Local identifier & $\begin{array}{l}\text { Station } \\
\text { number }\end{array}$ & Date & $\begin{array}{c}\text { Terbacil } \\
(\mu \mathrm{g} / \mathrm{L})\end{array}$ & $\begin{array}{c}\text { Terbufos } \\
(\mu \mathrm{g} / \mathrm{L})\end{array}$ & $\begin{array}{c}\text { Thiobencarb } \\
(\mu g / L)\end{array}$ & $\begin{array}{c}\text { Triallate } \\
(\mu \mathrm{g} / \mathrm{L})\end{array}$ & $\begin{array}{c}\text { Triclopyr } \\
(\mu \mathrm{g} / \mathrm{L})\end{array}$ & $\begin{array}{c}\text { Trifluralin } \\
(\mu \mathrm{g} / \mathrm{L})\end{array}$ \\
\hline BX-30-55-936 & 320939099093801 & $12-18-00$ & $<0.034$ & $<0.017$ & $<0.0048$ & $<0.0023$ & $<0.07$ & $<0.009$ \\
\hline DA-06-28-202 & 353704101343701 & $05-03-00$ & $<.007$ & $<.013$ & $<.002$ & $<.001$ & $<.25$ & $<.002$ \\
\hline DL-14-62-604 & 340406098160701 & $05-10-00$ & $<.007$ & $<.013$ & $<.002$ & $<.001$ & $<.25$ & $<.002$ \\
\hline DP-24-18-308 & 334352102450601 & 05-09-00 & $<.007$ & $<.013$ & $<.002$ & $<.001$ & $<.25$ & $<.002$ \\
\hline DP-25-24-304 & 334245103011001 & $05-08-00$ & $<.007$ & $<.013$ & $<.002$ & $<.001$ & $<.25$ & $<.002$ \\
\hline DU-12-06-609 & 345658100155501 & 05-08-00 & $<.007$ & $<.013$ & $<.002$ & $<.001$ & $<.25$ & $<.002$ \\
\hline DX-68-05-621 & 295520098242301 & $03-22-00$ & $<.007$ & $<.013$ & $<.002$ & $<.001$ & $<.25$ & $<.002$ \\
\hline DX-68-06-405 & 295528098222301 & $03-22-00$ & $<.007$ & $<.013$ & $<.002$ & $<.001$ & $<.25$ & $<.002$ \\
\hline DY-41-13-307 & 315105098241401 & 04-05-00 & $<.007$ & $<.013$ & $<.002$ & $<.001$ & $<.25$ & $<.002$ \\
\hline $\mathrm{HH}-45-35-505$ & 312715102402201 & $01-12-00$ & $<.007$ & $<.013$ & $<.002$ & $<.001$ & $<.25$ & $<.002$ \\
\hline HP-12-62-802 & 340210100184901 & $05-05-00$ & $<.007$ & $<.013$ & $<.002$ & $<.001$ & $<.25$ & $<.002$ \\
\hline HS-28-09-404 & 324810101591201 & $05-16-00$ & $<.007$ & $<.013$ & $<.002$ & $<.001$ & $<.25$ & $<.002$ \\
\hline HT-07-63-204 & 350607102103401 & $05-03-00$ & $<.007$ & $<.013$ & $<.002$ & $<.001$ & $<.25$ & $<.002$ \\
\hline HZ-77-34-606 & 282608099455301 & $03-28-00$ & $<.007$ & $<.013$ & $<.002$ & $<.001$ & $<.25$ & $<.002$ \\
\hline JA-05-57-613 & 350254100535501 & $05-04-00$ & $<.007$ & $<.013$ & $<.002$ & $<.001$ & $<.25$ & $<.002$ \\
\hline JA-12-12-404 & 344910100360401 & $05-02-00$ & $<.007$ & $<.013$ & $<.002$ & $<.001$ & $<.25$ & $<.002$ \\
\hline JH-45-05-924 & 315245102240201 & $05-25-00$ & $<.007$ & $<.013$ & $<.002$ & $<.001$ & $<.25$ & $<.002$ \\
\hline JL-49-04-116 & 315757106370201 & $01-06-00$ & $<.007$ & $<.013$ & $<.002$ & $<.001$ & $<.25$ & $<.002$ \\
\hline JL-49-04-423 & 315708106362301 & $01-03-00$ & $<.06$ & $<.013$ & $<.002$ & $<.001$ & $<.25$ & $<.002$ \\
\hline JL-49-04-428 & 315517106361401 & $01-06-00$ & $<.007$ & $<.013$ & $<.002$ & $<.001$ & $<.25$ & $<.002$ \\
\hline JL-49-04-492 & 315623106360601 & $01-06-00$ & $<.007$ & $<.013$ & $<.002$ & $<.001$ & $<.25$ & $<.002$ \\
\hline JL-49-22-626 & 314145106163601 & $01-04-00$ & $<.007$ & $<.013$ & $<.002$ & $<.001$ & $<.25$ & $<.002$ \\
\hline $\mathrm{JL}-49-24-423$ & 314107106063301 & $01-07-00$ & $<.007$ & $<.013$ & $<.002$ & $<.001$ & $<.25$ & $<.002$ \\
\hline JL-49-40-502 & 312648106044701 & $01-04-00$ & $<.007$ & $<.013$ & $<.002$ & $<.001$ & $<.25$ & $<.002$ \\
\hline JY-65-26-520 & 293314095474702 & $04-03-00$ & $<.007$ & $<.013$ & $<.002$ & $<.001$ & $<.25$ & $<.002$ \\
\hline KD-27-01-612 & 325523102542701 & $05-22-00$ & $<.007$ & $<.013$ & $<.002$ & $<.001$ & $<.25$ & $<.002$ \\
\hline KK-57-42-709 & 301545098502801 & $07-06-00$ & $<.007$ & $<.013$ & $<.002$ & $<.001$ & $<.25$ & $<.002$ \\
\hline KK-57-50-325 & 301357098471901 & $03-28-00$ & $<.007$ & $<.013$ & $<.002$ & $<.001$ & $<.25$ & $<.002$ \\
\hline KK-57-51-303 & 301411098383101 & $03-21-00$ & $<.007$ & $<.013$ & $<.002$ & $<.001$ & $<.25$ & $<.002$ \\
\hline KK-57-52-107 & 301430098363401 & $03-21-00$ & $<.007$ & $<.013$ & $<.002$ & $<.001$ & $<.25$ & $<.002$ \\
\hline
\end{tabular}


Table 5. Concentrations of soluble pesticides in public water-supply wells sampled in Texas, November 1999-January 2001-Continued

\begin{tabular}{|c|c|c|c|c|c|c|c|c|}
\hline Local identifier & $\begin{array}{l}\text { Station } \\
\text { number }\end{array}$ & Date & $\begin{array}{c}\text { Terbacil } \\
(\mu \mathrm{g} / \mathrm{L})\end{array}$ & $\begin{array}{c}\text { Terbufos } \\
(\mu \mathrm{g} / \mathrm{L})\end{array}$ & $\begin{array}{c}\text { Thiobencarb } \\
(\mu \mathrm{g} / \mathrm{L})\end{array}$ & $\begin{array}{c}\text { Triallate } \\
(\mu \mathrm{g} / \mathrm{L})\end{array}$ & $\begin{array}{c}\text { Triclopyr } \\
\text { ( } \mu \mathrm{g} / \mathrm{L})\end{array}$ & $\begin{array}{l}\text { Trifluralin } \\
(\mu \mathbf{g} / \mathbf{L})\end{array}$ \\
\hline KS-05-52-105 & 351428100352601 & 05-04-00 & $<.007$ & $<.013$ & $<.002$ & $<.001$ & $<.25$ & $<.002$ \\
\hline KT-18-11-406 & 334840096434001 & 04-19-00 & $<.007$ & $<.013$ & $<.002$ & $<.001$ & $<.25$ & $<.002$ \\
\hline KT-18-28-703 & 333039096362901 & 04-19-00 & $<.007$ & $<.013$ & $<.002$ & $<.001$ & $<.25$ & $<.002$ \\
\hline KY-11-49-512 & 341116101574601 & 05-04-00 & $<.007$ & $<.013$ & $<.002$ & $<.001$ & $<.25$ & $<.002$ \\
\hline $\mathrm{KY}-11-57-602$ & 340345101531801 & $05-03-00$ & $<.007$ & $<.013$ & $<.002$ & $<.001$ & $<.25$ & $<.002$ \\
\hline KZ-12-19-104 & 344234100431601 & 05-09-00 & $<.007$ & $<.013$ & $<.002$ & $<.001$ & $<.25$ & $<.002$ \\
\hline LD-13-44-907 & 341523099305701 & $12-19-00$ & $<.034$ & $<.017$ & $<.0048$ & $<.0023$ & $<.07$ & $<.009$ \\
\hline LJ-60-64-807 & 300149095045001 & 05-04-00 & $<.007$ & $<.013$ & $<.002$ & $<.001$ & $<.25$ & $<.002$ \\
\hline LP-21-49-317 & 331233099545201 & 05-01-00 & $<.007$ & $<.013$ & $<.002$ & $<.001$ & $<.25$ & $<.002$ \\
\hline LX-24-40-405 & 332624102072901 & $05-17-00$ & $<.007$ & $<.013$ & $<.002$ & $<.001$ & $<.25$ & $<.002$ \\
\hline LY-32-26-706 & 323204097500301 & $03-22-00$ & $<.007$ & $<.013$ & $<.002$ & $<.001$ & $<.25$ & $<.002$ \\
\hline LY-32-34-208 & 322958097481301 & $03-21-00$ & $<.007$ & $<.013$ & $<.002$ & $<.001$ & $<.25$ & $<.002$ \\
\hline LY-32-34-303 & 322928097461701 & 04-03-00 & $<.007$ & $<.013$ & $<.002$ & $<.001$ & $<.25$ & $<.002$ \\
\hline LY-32-34-510 & 322537097485701 & $03-21-00$ & $<.007$ & $<.013$ & $<.002$ & $<.001$ & $<.25$ & $<.002$ \\
\hline LY-32-34-609 & 322626097454801 & $03-21-00$ & $<.007$ & $<.013$ & $<.002$ & $<.001$ & $<.25$ & $<.002$ \\
\hline PB-28-62-105 & 320649101215501 & 04-26-00 & $<.007$ & $<.013$ & $<.002$ & $<.001$ & $<.25$ & $<.002$ \\
\hline PK-43-50-216 & 311422100490201 & $12-20-00$ & $<.034$ & $<.017$ & $<.0048$ & $<.0023$ & $<.07$ & $<.009$ \\
\hline PS-52-02-404 & 305502103504101 & 01-10-00 & $<.007$ & $<.013$ & $<.002$ & $<.001$ & $<.25$ & $<.002$ \\
\hline PU-84-43-504 & 271841098402801 & 04-04-00 & $<.007$ & $<.013$ & $<.002$ & $<.001$ & $<.25$ & $<.002$ \\
\hline PX-32-47-109 & 322201097141601 & $03-20-00$ & $<.007$ & $<.013$ & $<.002$ & $<.001$ & $<.25$ & $<.002$ \\
\hline
\end{tabular}


Table 5. Concentrations of soluble pesticides in public water-supply wells sampled in Texas, November 1999-January 2001-Continued

\begin{tabular}{|c|c|c|c|c|c|c|c|c|}
\hline Local identifier & $\begin{array}{l}\text { Station } \\
\text { number }\end{array}$ & Date & $\begin{array}{c}\text { Terbacil } \\
(\mu \mathrm{g} / \mathrm{L})\end{array}$ & $\begin{array}{c}\text { Terbufos } \\
(\mu \mathrm{g} / \mathrm{L})\end{array}$ & $\begin{array}{c}\text { Thiobencarb } \\
(\mu g / L)\end{array}$ & $\begin{array}{c}\text { Triallate } \\
(\mu \mathrm{g} / \mathrm{L})\end{array}$ & $\begin{array}{c}\text { Triclopyr } \\
(\mu \mathrm{g} / \mathrm{L})\end{array}$ & $\begin{array}{c}\text { Trifluralin } \\
(\mu \mathrm{g} / \mathrm{L})\end{array}$ \\
\hline RU-10-60-606 & 340427102302801 & $05-02-00$ & $<0.007$ & $<0.013$ & $<0.002$ & $<0.001$ & $<0.25$ & $<0.002$ \\
\hline RW-41-63-520 & 310238098104001 & $03-23-00$ & $<.007$ & $<.013$ & $<.002$ & $<.001$ & $<.25$ & $<.002$ \\
\hline SK-57-19-201 & 304323098421701 & 03-08-00 & $<.007$ & $<.013$ & $<.002$ & $<.001$ & $<.25$ & $<.002$ \\
\hline SK-57-34-201 & 303001098485201 & 03-09-00 & $<.007$ & $<.013$ & $<.002$ & $<.001$ & $<.25$ & $<.002$ \\
\hline SP-23-17-417 & 334043101585701 & $05-10-00$ & $<.007$ & $<.013$ & $<.002$ & $<.001$ & $<.25$ & $<.002$ \\
\hline SP-23-18-107 & 334306101504101 & $05-10-00$ & $<.007$ & $<.013$ & $<.002$ & $<.001$ & $<.25$ & $<.002$ \\
\hline SP-23-19-812 & 333955101404601 & $05-10-00$ & $<.007$ & $<.013$ & $<.002$ & $<.001$ & $<.25$ & $<.002$ \\
\hline SP-24-24-214 & 334419102045201 & $05-18-00$ & $<.007$ & $<.013$ & $<.002$ & $<.001$ & $<.25$ & $<.002$ \\
\hline SR-23-41-602 & 331933101543701 & $05-15-00$ & $<.007$ & $<.013$ & $<.002$ & $<.001$ & $<.25$ & $<.002$ \\
\hline SS-42-52-504 & 311202099321401 & $03-01-00$ & $<.007$ & $<.013$ & $<.002$ & $<.001$ & $<.25$ & $<.002$ \\
\hline ST-40-39-304 & 312745097083301 & $03-23-00$ & $<.007$ & $<.013$ & $<.002$ & $<.001$ & $<.25$ & $<.002$ \\
\hline SY-27-39-505 & 322555102105501 & $05-24-00$ & $<.007$ & $<.013$ & $<.002$ & $<.001$ & $<.25$ & $<.002$ \\
\hline SY-28-42-803 & 321619101495001 & $05-23-00$ & $<.007$ & $<.013$ & $<.002$ & $<.001$ & $<.25$ & $<.002$ \\
\hline SY-28-50-908 & 320816101474601 & $05-23-00$ & $<.007$ & $<.013$ & $<.002$ & $<.001$ & $<.25$ & $<.002$ \\
\hline TB-76-03-605 & 285657100373801 & $03-28-00$ & $<.007$ & $<.013$ & $<.002$ & $<.001$ & $<.25$ & $<.002$ \\
\hline TJ-27-63-712 & 320143102123101 & $12-20-00$ & $<.034$ & $<.017$ & $<.0048$ & $<.0023$ & $<.07$ & $<.009$ \\
\hline TJ-28-57-702 & 320221101594901 & $05-25-00$ & $<.007$ & $<.013$ & $<.002$ & $<.001$ & $<.25$ & $<.002$ \\
\hline TJ-28-57-903 & 320010101523701 & $05-23-00$ & $<.007$ & $<.013$ & $<.002$ & $<.001$ & $<.25$ & $<.002$ \\
\hline TJ-45-07-407 & 315643102131101 & $05-24-00$ & $<.007$ & $<.013$ & $<.002$ & $<.001$ & $<.25$ & $<.002$ \\
\hline TJ-45-07-606 & 315702102075401 & $05-25-00$ & $<.007$ & $<.013$ & $<.002$ & $<.001$ & $<.25$ & $<.002$ \\
\hline TS-60-36-510 & 302701095331201 & 04-20-00 & $<.007$ & $<.013$ & $<.002$ & $<.001$ & $<.25$ & $<.002$ \\
\hline TW-22-02-714 & 335416100502101 & $05-05-00$ & $<.007$ & $<.013$ & $<.002$ & $<.001$ & $<.25$ & $<.002$ \\
\hline TW-22-02-716 & 335449100513101 & $12-19-00$ & $<.034$ & $<.017$ & $<.0048$ & $<.0023$ & $<.07$ & $<.009$ \\
\hline UJ-62-49-712 & 300906093585201 & 04-28-00 & $<.007$ & $<.013$ & $<.002$ & $<.001$ & $<.25$ & $<.002$ \\
\hline UK-31-05-501 & 325545098255501 & $03-22-00$ & $<.007$ & $<.013$ & $<.002$ & $<.001$ & $<.25$ & $<.002$ \\
\hline UK-31-24-501 & 324210098034701 & $03-22-00$ & $<.007$ & $<.013$ & $<.002$ & $<.001$ & $<.25$ & $<.002$ \\
\hline UL-35-48-202 & 322207094034501 & $04-25-00$ & $<.007$ & $<.013$ & $<.002$ & $<.001$ & $<.25$ & $<.002$ \\
\hline UP-32-10-604 & 324926097455001 & 02-09-00 & $<.007$ & $<.013$ & $<.002$ & $<.001$ & $<.25$ & $<.002$ \\
\hline UP-32-11-103 & 325005097440101 & 02-09-00 & $<.007$ & $<.013$ & $<.002$ & $<.001$ & $<.25$ & $<.002$ \\
\hline UP-32-11-709 & 324649097442001 & 02-09-00 & $<.007$ & $<.013$ & $<.002$ & $<.001$ & $<.25$ & $<.002$ \\
\hline
\end{tabular}


Table 5. Concentrations of soluble pesticides in public water-supply wells sampled in Texas, November 1999-January 2001-Continued

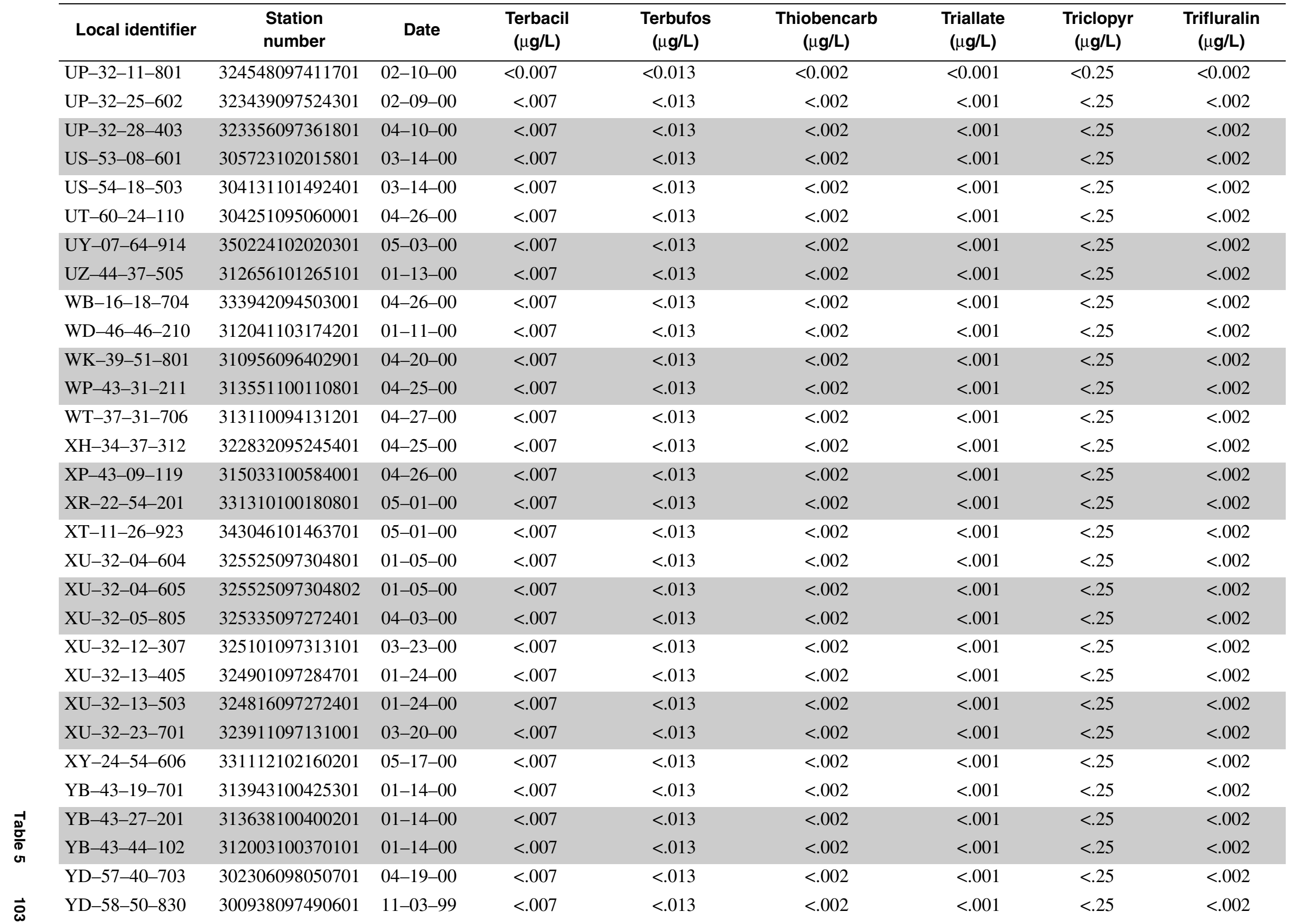


Table 5. Concentrations of soluble pesticides in public water-supply wells sampled in Texas, November 1999-January 2001-Continued

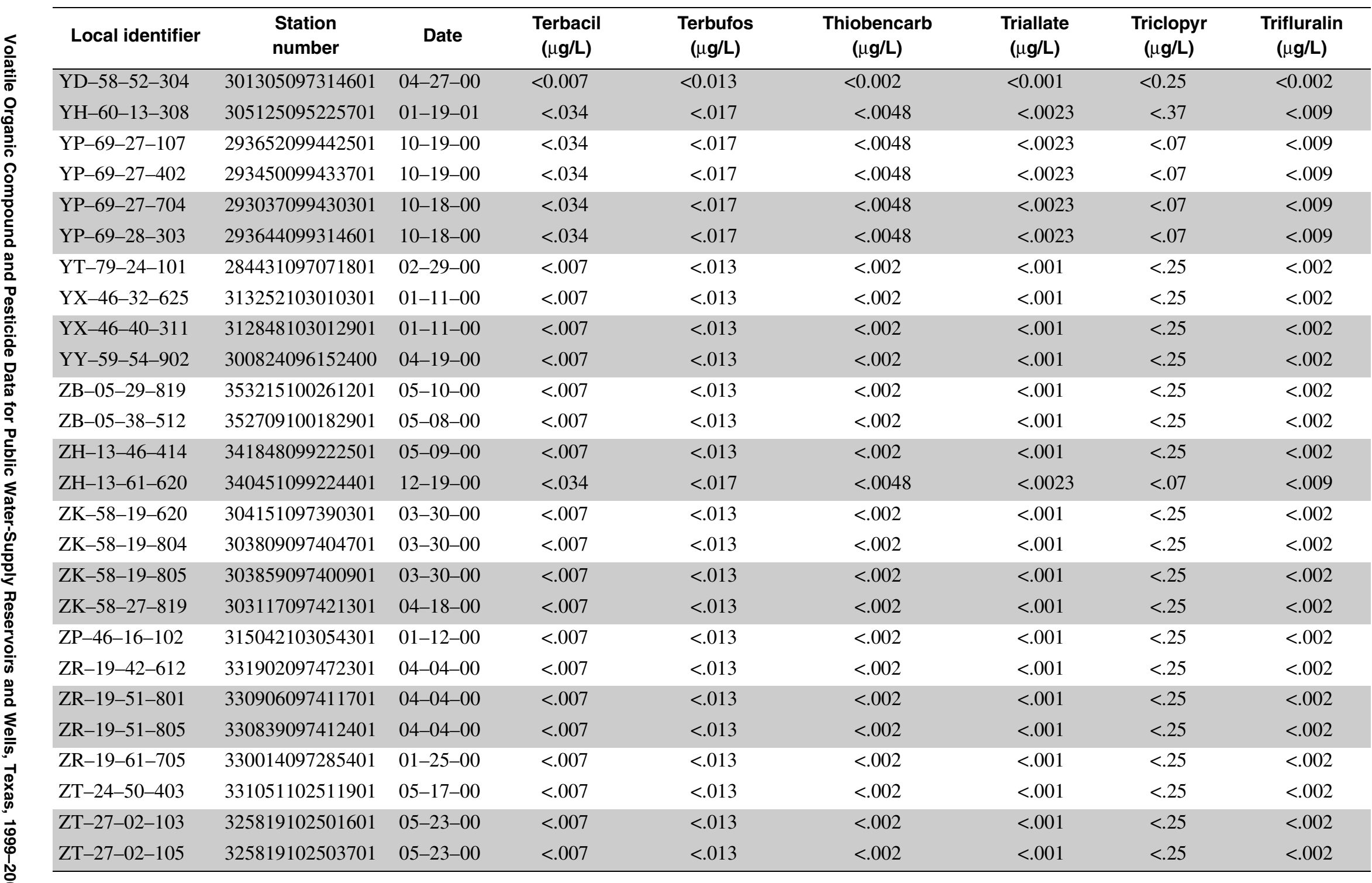

${ }^{1}$ Some results not available due to breakage at laboratory. 
Table 6. Summary of soluble pesticides in samples collected from public water-supply wells in Texas, November 1999January 2001 (only pesticides with one or more detections are shown)

[ $\mu \mathrm{g} / \mathrm{L}$, micrograms per liter; MCL, maximum contaminant level; --, not applicable]

\begin{tabular}{|c|c|c|c|c|}
\hline Pesticide & $\begin{array}{l}\text { Method detection limit } \\
\qquad(\mu \mathrm{g} / \mathrm{L})\end{array}$ & $\begin{array}{c}\text { Detection frequency } \\
\text { (percent) }\end{array}$ & $\begin{array}{l}\text { Maximum concentration } \\
(\mu \mathrm{g} / \mathrm{L})\end{array}$ & $\begin{array}{c}\text { MCL } \\
(\mu g / L)\end{array}$ \\
\hline Deethylatrazine & 0.002 & 22 & 1.73 & -- \\
\hline Atrazine & .001 & 16 & 1.54 & 3.0 \\
\hline Prometon & .018 & 13 & .117 & -- \\
\hline Simazine & .005 & 5.2 & .085 & 4.0 \\
\hline Diazinon & .002 & 4.0 & .025 & -- \\
\hline Bromacil & .06 & 2.9 & .450 & -- \\
\hline Picloram & .05 & 2.3 & .581 & 500 \\
\hline Tebuthiuron & .01 & 2.3 & .067 & -- \\
\hline Fenuron & .07 & 1.7 & .16 & -- \\
\hline Metolachlor & .002 & 1.7 & .005 & -- \\
\hline Dicamba & .043 & 1.1 & .060 & -- \\
\hline Diuron & .06 & 1.1 & .14 & -- \\
\hline Dacthal mono-acid & .039 & 6 & .04 & -- \\
\hline Alachlor & .002 & 6 & .006 & 2.0 \\
\hline Bentazon & .035 & 6 & .049 & -- \\
\hline$p, p^{\prime}-\mathrm{DDE}$ & .0025 & .6 & .002 & -- \\
\hline
\end{tabular}

